\title{
Thinking the worst about pain
}

Citation for published version (APA):

Severeijns, J. R. M. (2005). Thinking the worst about pain. [Doctoral Thesis, Maastricht University]. Datawyse / Universitaire Pers Maastricht. https://doi.org/10.26481/dis.20050421js

Document status and date:

Published: 01/01/2005

DOI:

10.26481/dis.20050421js

Document Version:

Publisher's PDF, also known as Version of record

\section{Please check the document version of this publication:}

- A submitted manuscript is the version of the article upon submission and before peer-review. There can be important differences between the submitted version and the official published version of record.

People interested in the research are advised to contact the author for the final version of the publication, or visit the DOI to the publisher's website.

- The final author version and the galley proof are versions of the publication after peer review.

- The final published version features the final layout of the paper including the volume, issue and page numbers.

Link to publication

\footnotetext{
General rights rights.

- You may freely distribute the URL identifying the publication in the public portal. please follow below link for the End User Agreement:

www.umlib.nl/taverne-license

Take down policy

If you believe that this document breaches copyright please contact us at:

repository@maastrichtuniversity.nl

providing details and we will investigate your claim.
}

Copyright and moral rights for the publications made accessible in the public portal are retained by the authors and/or other copyright owners and it is a condition of accessing publications that users recognise and abide by the legal requirements associated with these

- Users may download and print one copy of any publication from the public portal for the purpose of private study or research.

- You may not further distribute the material or use it for any profit-making activity or commercial gain

If the publication is distributed under the terms of Article $25 \mathrm{fa}$ of the Dutch Copyright Act, indicated by the "Taverne" license above, 


\section{THINKING THE WORST ABOUT PAIN}

THE ROLE OF PAIN CATASTROPHIZING IN (CHRONIC) PAIN 


\section{COLOPHON}

Cover: $\quad$ Edvard Munch, The Scream, 1893

(C) c/o Beeldrecht Amsterdam 2005

Cover design: Ruud Severeijns

Printed by: Datawyse, Maastricht

R. Severeijns, Maastricht 2005

ISBN: 90-9019232-8 


\section{THINKING THE WORST ABOUT PAIN}

THE ROLE OF PAIN CATASTROPHIZING IN (CHRONIC) PAIN

\section{PROEFSCHRIFT}

ter verkrijging van de graad van doctor

aan de universiteit Maastricht.

op gezag van de Rector Magnificus,

Prof. Mr. G.P.M.F. Mols

volgens het besluit van het College van Decanen,

in het openbaar te verdedigen op

donderdag 21 april 2005 om 14.00 wur

door

Johannes Rudolf Maria Severeijns 
PROMOTOR:

Prof. Dr. M.A. van den Hout

COPROMOTOR:

Dr. I.W.S. Vlaeyen

BEOORDELINGSCOMMISSIE:

Prof. Dr. A. Anntz (voorzitter)

Prof. Dr. G. Crombez (Uniwersiteit wan Gent, België)

Dr. J. Patijn

Di. T. Schmidt

Prof. Dr. M. Sullivan (University of Montreal, Canada) 
Catastrophe. (kattotstrofin). Also catastrophy. [a. Gr. karowrpophn

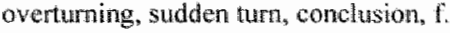

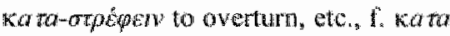
down + atoferen to turn.]

1. The change or revolution which produces the conclusion or final event of a dramatic piece' (I.); the dènouennent.

2. 'A final event; a conclusion generally unthappy' (J.); a disastrous end, finish-up, conclusion, upshot; overthrow, ruin, callamitous fate.

3. An event producing a subversion of the order or system of things.

b. esp. in Geol. A sudden and wiolent change iri the physical order of things, such as a sudden upheaval, depression, or convulsion affecting the earth"s surface, and the living beings upon it, by which sone have supposed that the suecessive geological periods were suddenly brought to an end. (Cf. CATACLYSM, CATASTROPHISM M

4. A sudden diswster, wide-spread, very fatal, or signal. (In the application of exaggerated language to misfortunes it is used very loosely.)

The Oxford English Dictionary, 1933 



\section{Table of Contents}

\section{Chapter 1}

General introduction

\section{Chapter 2}

(Chronic) pain: Towards a cognitive-behavioral perspective

\section{Chapter 3}

Pain catastrophizing

\section{Chapter 4}

Pain catastrophizing predicts pain intensity, disability, and psychological distress independent of the level of plysical impairment

\section{Chapter 5}

Pain catastrophizing and general health status in a large Dutch community sample

\section{Chapter 6}

Pain catastrophizing is associated with health indices in musculoskeletal pain: A cross-sectional study in the Dutch community

\section{Chapter 7}

Pain catastrophizing and consequences of musculoskeletal pain: A prospective study in the Dutch community

\section{Chapter 8}

The causal status of pain catastrophizing: An experimental test with healthy participants

\section{Chapter 9}

The causal status of pain catastrophizing: An experimental test with chronic low back pain patients

\section{Chapter 10}

General discussion

References

Summary

Samenvatting

Dankwoord

About the author 



\section{CHAPTER 1}

General introduction

"Pain is no evil unless it conquers us"

George Elliot 


\section{General introduction}

In this general introductory Chapter, some of the frequently used terms concerning pain and pain research will be defined and some epidemiological data with respect to the prevalence and consequences of (chronic) pain will be discussed. Also, a historical overview of the role of psychological factors in the study of pain will be presented.

\section{Definition of terms}

Pain, especially in its acute form, is a common experience to all of us. It is thought to have an important biological function by signaling impending or already present tissue damage and instigating the individual to take action to prevent further damage or to seek adequate treatment or help. Although usually associated with and caused by an injury, a disease, or a systemic or functional disorder, in some persons it can become persistent in the absence of apparent biomedical findings or beyond expected healing time and contribute to an often significant decrease in the quality of life (Miller \& Kraus, 1990).

Notwithstanding its common occurrence, pain is still badly understood. Being a construct or a concept, its definition is a function of the theoretical orientation one takes regarding pain. In this respect pain may mean something different for, lets say a neurosurgeon than for a psychologist and even within disciplines there is not much consensus about any particular pain with respect to etiology, patho-physiology, and treatment (Boyd, 1989; Feuerstein, 1989). A nowadays widely accepted definition of pain is the definition proposed by the International Association for the Study of Pain (IASP) in which pain is defined as "an unpleasant sensory and emotional experience associated with actual or potential tissue damage, or described in terms of such damage" (Mersky, 1986). In this definition pain is emphasized as a sensory and emotional experience and is not exclusively defined in terms of nociceptive input, the latter referring to peripheral sensory stimulation that is capable of being perceived as pain (Turk, 1996b).

Traditionally, pain complaints are classified on the basis of the duration of the complaints and a distinction is made between acute pain and chronic pain. Acute pain is most often defined as pain complaints lasting to a maximum of six weeks. Chronic pain is rather arbitrarily defined as pain that persists for more than 3 months (Goossens \& van Tulder, 2000 ), although a period of six months (Miller \& Kraus, 1990) is also used very often. However, it is questionable whether it is meaningful to make the distinction between acute and chronic pain. The majority of the people with back pain for example, experience recurrent episodes of back pain, which in general are more frequent and more serious if they have had manifold or prolonged back pain complaints in the past. Therefore, it might be more important to carefully map these relapses of back pain than to stress the duration of the complaints (Goossens \& van Tulder, 2000; Von Korff \& Saunders, 1996). Nevertheless, the distinction between acute and chronic pain is still being made in contemporary pain research and will be adhered to in the current thesis as well.

Because contemporary (chronic) pain management focuses on restoration of function instead of lessening or easing pain itself, definitions of function should also be considered (Feuerstein, 1989). Three broad categories are used to define alterations in functional capacity, each category describing a different level of functioning respectively. The first category concerns the more organic, physical level of functioning (e.g., being able or unable to walk), the second category concerns the level of skills (e.g., the ability or inability to gardening or driving a car), and the third category refers to the different roles in life (e.g., being a father/mother or an employee). The following constructs are used to refer to these categories: "impairment", "disability" or "functional limitation", and "handicap". 
Operationalizations of these constructs are often used as (outcome) vrariables in pain research. As with the definition of chronic pain, these constructs are not defined in a uniform way and are frequently used interchangeably, making a direct comparison between different studies sometimes rather difficult. In Table 1 two sets of definitions are shown: the International Classification of Impairments, Disabilities, and Handicaps (ICIDH) of the World Health Organization (WHO, 1980) and the definitions of the Committee on Pain, Disability, and Chronic Illness Behavior of the Institute of Medicine (Feuerstem, 1989; Osterweis, Kleinman \& Mechanic, 1987). In recent years the conceptualization of these constructs has changed considerably. This is illustrated quite well in the classification system of the WHO. The WHO's ICIDH (WHO, 1980) is a "consequences of disease" classification and the previously mentioned constructs are conceptualized as the impacts or consequences of disease or other health conditions. The $200 \rrbracket$ revision of the ICIDH (i.e., the International Classification of Functioning, Disability, and Health: ICF) has moved away from being a "consequences of disease" classification to become a "components of health" classification (WHO, 2001). In this respect, the ICF does not pronounce upon etiology and the constructs of impairment, disability, and handicap are used in a different way as in the ICIDH. Elaborating on the ICF however is beyond the scope of the current thesis, all the more because the vast majority, if not all, of the studies referred to, either explicitly or implicitly still employ the definitions shown in Table 1.

Table 1 .

Definitions of function (feuerstein, 1989; Osterweis et al., 1987; WHO, 1980)

\begin{tabular}{|c|c|c|c|}
\hline Category & definition & construct & Detinition by: \\
\hline 1 & $\begin{array}{l}\text { Any loss or abnormality of } \\
\text { psychological, phystiological or anatomic } \\
\text { structure or function }\end{array}$ & impairment & WHOO $/ \mathrm{MM}^{\mathrm{b}}$ \\
\hline \multirow[t]{2}{*}{2} & $\begin{array}{l}\text { Any restriction or lack of ability to } \\
\text { perform an activity in the manner or } \\
\text { within the range considered nomal for a }\end{array}$ & Disability & WHO \\
\hline & $\begin{array}{l}\text { human being that results from an } \\
\text { impaument }\end{array}$ & Functional limitation & $1 \mathrm{M}$ \\
\hline \multirow{2}{*}{3} & $\begin{array}{l}\text { A disadvantage for a given individual } \\
\text { (nesulting from an impairment or } \\
\text { functional limitation) that limits or }\end{array}$ & Handicap & WHO \\
\hline & $\begin{array}{l}\text { prevents the fullilment of a role that is } \\
\text { nombut (funetion of age, sex social and } \\
\text { culfural lactors) for that individual }\end{array}$ & disability & IM \\
\hline
\end{tabular}

\footnotetext{
"World Haalth Organization.

"Committee on Pain, Disability, and Chronic IIness Bethavion of the Institute of Medicine.
}

\section{Prevalence and consequences of chronic pain}

As mentioned previously, acute pain is a common experience to all of us. Estimates of the prevalence of chronic pain, however, vary considerably, depending on the differences in the definitions of chronic pain that are used (cf:, Crombie, Davies \& Macrae, 1994; Von Korf, Dworkin \& le Resche, 1990), the type of pain included in the study, the survey methods, and the population the data are taken from. Nevertheless, international epidemiological studies have shown that in general chronic pain presents a major public health problem, with estimates of its prevalence ranging from $4 \%$ up to $40 \%$ (Andersson, 1994; Bowsher, Rigge \& Sopp, 1991; Brattberg, Thorslund \& Wikman, 1989; Crook, Rideout \& Browne, 1984; 
Feuerstein, Papciak \& Hoon, 1987; Magni, Caldheron, Rigatti Luchini \& Merskey, 1990; Sternbach, 1986).

An epidemiological study in the Netherlands (Picavet, Schouten \& Smit 1996) concluded that the report of chronic complaints in the Dutch population increases from $12 \%$ in the age bracket $20-29$ years old to $21.1 \%$ in the age bracket $50-59$ years old. More recently, as much as $44.4 \%$ of the participants in a Dutch population-based study on musculoskeletal pain reported a chronic musculoskeletal pain complaint (Picavet, van Gils \& Schouten, 2000).

The detrimental consequences of chronic pain in terms of impairment and disability have a tremendous socio-economic impact due to the enormous health-care costs, loss of wages and productivity, costs of disability benefits and compensation (Bowsher et al.; 1991; Feverstein et al., 1987; Latham \& Davis, 1994; Sternbach, 1986; Turk, 1996b; Turk, Meichenbaum \& Genest, 1983; van Tulder, Koes \& Bouter, 1995). This can be illustrated for the Netherlands: in 1991, the total costs of back complaints were estimated at well over 9 billion guilders which corresponds with $1.7 \%$ of the Gross National Product (van Tulder et al., 1995; van Tulder, van Breukelen, Koes \& Bouter, 1997). These costs can be divided in direct costs (healthcare, medical, and patient-related costs) and indirect costs (loss of productivity and absenteeism) and were estimated at almost 700 million guilders and 8.6 billion guilders respectively. Remarkably, about $90 \%$ of the total costs for back complaints are indirect, whereas, normally, direct and indirect costs make up about $70 \%$ and $30 \%$ of total health care costs, respectively (Goossens \& van Tulder, 2000). Furthermore, it seems that a relatively small proportion of back pain patients account for most of the costs: the permanently disabled, i.e., the patients with chronic back pain represent less than $10 \%$ of all but nevertheless account for $85 \%$ of all costs associated with the illness and account for $90 \%$ of lost productivity (Cats-Baril \& Frymoyer, 1991).

Also, the quality of life is adversely influenced by a chronic pain condition, as illustrated. by considerable impairments (e.g., restricted walking or mobility), comparatively high disability levels for everyday activities, recreation, social interaction, sleep and rest, and the association of emotional states of anger, demoralization, anxiety, and depression with chronic pain conditions (Latham \& Davis, 1994; Magni et al., 1990; Reesor \& Craig, 1988).

\section{The pain concept: historical overview and the role of psychological factors in (chronic) pain}

Today, it is generally recognized that psychological factors play an important role in (chronic) pain. However, this has not always been so. Over the years the role attributed to psychological factors has varied considerably, along with changes in medical and scientific methods and paradigms (Gamsa, 1994a). Of particular rellevance to the study of pain is an old-established and even today still important and controversial issue in psychology relating to the body-mind relationship. Over the last thousand years various conceptualizations of the body-mind relationship have given rise to the development of different forms of psychophysics and related views on the nature of pain. A conceptualization that has been quite influential is the naturalism of the classical Greek philosophy and science, in which nature or matter was seen as the cause of pain. This naturalism returned during the Renaissance period in the writings of Descartes who saw body and soul as separate but interacting entities. The relationship between the two however, was purely physical and pain was characterized as a simple reflex response to a physical stimulus (Waddell, 1987). The ideas of Descartes were further elaborated by Leibnitz and later by Kant (see Schneider \& Karoly, 1983) and formed the basis of the physical reductionism of the first modern theories on pain (e.g., the specificity theory, formulated by Mueller in 1826 and extended by Frey in 1895, several pattern theories, central summation theory as proposed by Goldscheider in 1894 and sensory interaction theory, proposed by Noordenbos in 1959), in which the degree of tissue damage was expected to be 
direetly related to the severity of pain and the nature of pain could only be defined by identifying the related physiological mechanisms. Although there were early modern writers who referred to the psychological and psychiatric aspects of pain, by the second half of the nineteenth century the most prevailing opinion was that all pains were the direct result of tissue damage and thus of organic origin (Gamsa, 1994a). These views fitted in well with the rising medical model of disease, the industrial revolution, and the enormous technological progression after World War II which contributed significantly to the control of disease (Waddell, 1987).

By the second half of the 20 th century it became increasingly clear however, that not all pain phenomena could be accounted for by physical explanations (Gamsa, 1994a). More specifically, there are some puzzling and perplexing features of (persisting) pain complaints that do not fit the bionedical model which assumes a close and direct correspondence between a biological state (e.g., an injury, a disease, or some other health condition) and the experience of pain. Evidence for the often highly variable and unpredictable link between injury and pain is abundant. Some of these, often striking anomalies with the medical model will be discussed here in some detail!

Beecher (1959) for example observed the behavior of soldiers who were severely wounded in battle during World War II and found that not less than $65 \%$ of them did not report any significant pain for hours or even days after the injury and refused any medication to relieve it. They were not in a state of shock and indeed were able to feel pain for they complained of pain when receiving a vein puncture. After the war, in clinical practice, he found that some $20 \%$ of civilians who underwent major surgery reported little or no pain for hours or days after the injury or incision and did not require pain medication. These observations suggest that there is no simple and direct relationship between the wound per se and the pain experienced. Beecher explained these striking observations by hypothesizing that if is the subjective significance of the wound or the meaning of the situation, which greatly influences the degree and the quality of the pain that people feel. For example, for the wounded soldiers their injury meant escape from the battlefield and return to a safe environment.

Another illustration of the importance of the meaning of noxious stimuli can be found in the conditioning experiments carried out by Bavlov. In a series of experiments he found that dogs could be conditioned to react to a noxious stimulus such as a strong electric current or wounding or cauterization of the skin, with an alimentary conditioned reflex (turning its head to the place where it normally received food, salivating, smacking its lips), where normally these stimuli would evoke vigorous unconditioned defense reflexes (Anrep, 1960).

Furthermore, there are cases of congenital insensitivity to pain, of which $M$ iss $C$ is the best documented one (McMurray, 1950). In an experimental study, McMurray (1950) conducted a number of experiments using different pain-producing stimuli (immersion of the right hand in ice water, immersion of the right hand in hot water, and electric shock). In contrast to the control subjects, Miss C. showed a total lack of response to these common pain-producing stimuli. More specifically, there was no verbal report of painful feelings, there were no behavioral signs (e.g., yocal expressions, withdrawal), and most surprisingly, there was no physiological response (as seen in the blood pressure, heart rate, and respiratory measures). These fundings suggest an organic defect. At the age of 29, Miss $\mathrm{C}$. died of massive infections that could not be controlled. Post mortem examination of her nervous system however, failed to show any abnormality (Melzack \& Wall, 1982). Although it is evident now that congenital insensitivity to pain may be due to many causes and evidence of neurological damage is found

\footnotetext{
For a more extensive description of these anomalies, the importance of psychological factors such as attention, past experience, perceived control etc, and a discussion of the clinical and psychological evidence aginst the specitioity theory and the pattern theores see Melzack and Wall (1982) and Melzack and Wall $(1965)$
} 
in some cases, the findings on post mortem examination may still be puzzling and contradictory (see Melzack \& Wall, 1982).

On the other hand there are cases of pain dispropontionate to the severity of the injury or in the apparent absence of noxious stimulation. Melzack and Wall (1982) describe the typical reaction of people who experience the passing of a kidney stone, an event that occurs in a poorly innervated structure, while the kidney stone is often not more than twice the size of the urethra which makes it a rather trivial event in mechanical terms: "Dwring this process. agonizing spasms of pain sweep over the patient so that the toughest and most stoical of characters usually collapses. The patien is pale, with a racing pulse, knees drawn up with a rigid abdominal wall and motionless. Even crying out is restraned becouse all movenew exaggerates the pain. As the stone passes into the bladder, there is immediate and complete relief, leaving a dazed and exhausted patient".

Parry (1980) studied over 100 cases of avulsion lesions of the brachial plexus, a network of nerves innervating the arns. Injuries of this kind mostly result from serious motor accidents, leaving the afflicted limb totally paralyzed and anesthetic. Nevertheless, most of these patients complain of prolonged severe pain which is consistently described as a burning pain. Furthermore, the majority of them also suffer from periodic sharp paroxysms of pain usually lasting a few seconds, sometimes of unbearable intensity. The pain can last for years after the original injury and is highly resistant to any form of therapy (Parry, 1980).

These examples illustrate perfectly well that the traditional biomedical model of pain fals short of explaining the complexities and peculiarities of (chronic) pain. The shortcomings of the biomedical model are concisely summarized by Turk (1996b):

1. patients with objectively determined equivalent degrees and types of tissue pathology vary widely in their reports of pain severity

2. asymptomatic individuals often reveal objective radiographic evidence of struchural abnormalities

3. conversely, patients with minimal objective physical pathology often complain of severe pain

4. surgical procedures designed to inhibit symptoms by severing neurological pathways believed to be subserving the reported pain may fail to alleviate pain

5. patients with objectively equivalent degrees of tissue pathology and treated with identical interventions respond in widely disparate ways

6. there are only low to modest correlations among physical impaiment, physical functioning, pain report, disability, and response to rehabilitation.

As a result of the inadequacy of the biomedical model and as the recognition of the disciplines of psychology and psychiatry grew, there became more acceptance of the importance of psychological factors in pain and several psychological models of chronic pain were put forward ${ }^{2}$. All the same, for well into the middle of the 20 th century pain was believed to be either organic or psychological in origin and the psychological explanations of pain were based on linear causal models. It was not untill the mid sixties that Melzack and Wall introduced the gate-control theory of pain, in which psychological and physiological factors were comprehensively integrated and mind and body were seen as mutually interactive (Melzack \& Casey, 1968; Melzack \& Totgerson, 1971; Melzack \& Wall, 1965; Melzack \& Wall, 1982). The gate-control theory provided the physiologic basis for the biopsychosocial model in which pain is not merely regarded as a physical sensation of noxjous stimulus and

\footnotetext{
${ }^{2}$ Notably the psychogenic vilew (e.g., the psychoamalytic model and a model of a "pain-prone" personality) and the motivational view. For a more elaborate discussion of these views and models see (Gamsa (1094a), Turk (1996b), Turk and Rudy (1992), and Turk and Rudy (1986).
} 
disease. Instead, the conscious experience of pain may be modulated by cognitive, emotional,

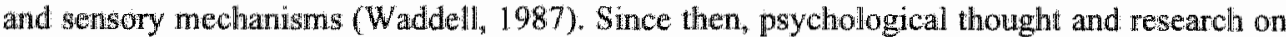
(chronic) pain has progressed, and today there is little convincing evidence to support dualistic conceptions which separate pain of psychological origin from physically generated pain in patients with chronic pain (Gamsa, 1994b). In addition there has been a shift from simple finear causality to muliticausal explanations and pain has come to be conceptualized as a complex phenomenon in which multiple physical and psychosocial factors interact (Gamsa, $1994 \mathrm{a}, 1994 \mathrm{~b})$. 


\section{CHAPTER 2}

(Chronic) pain: towards a cognitive-behavioral perspective

"If is easier to find men who will volunter to die, than ro find those who are willing to endure pain with patience"

Julius Cosesar 
18 


\section{Introduction}

There are three conceptual models of pain that have considerably influenced current conceptualizations and treatments of pain as well as current research on pain: the gatte-control theory (Melzack \& Casey, 1968; Melzack \& Torgerson, 1971; Melzack \& Wall, 1965. Melzack \& Wall, 1982), the operant conditioning theory (Fordyce, 1976), and the diathesisstress model as proposed by Flor, Birbanmer, and Turk (1990). As such, these models contributed highly to the development of the contemporary cognitive-behavioral perspective of pain. After a closer look at these theories, the cognitive-behavioral perspective will be explained and some findings that corroborate the importance of cognitive factors in the (chronic) pain experience will be discussed. Before further delineating the subject of this thesis, some shortcomings and limitations of the aforementioned models will be discussed.

\section{The gate-control theory of pain}

As stated before, Melzack and Wall's (1965) gate-control theory of pain was the first theory to comprehensively integrate psychological and physiological factors and as such, it brought the psychological study of pain into mainstream research (Gamsia 1994 a). For this reason and for the fact that it attributes an important role to cognitive-evaluative processes in the modulation of pain which in turn inspired cognitive approaches to the treatment of pain (Gamsa, 1994a), it will be discussed here in some detail.

The gate-control theory is based on a synthesis of concepts derived from the earlier specificity and pattern theories, and partly on the concept of selectivity of function ${ }^{3}$. Helped by the wave of new scientific facts and ideas in the 1960s, it was set up to explain and incorporate the numerous clinical observations and findings that demonstrated the often highly variable and unpredictable link between pain and injury, as well as the importance of psychological factors such as past experience, the meaning attached to a pain-producing situation, attention and anxiety etc. In the original 1965 formulation, the gate-control theory basically proposes a neural mechanism in the dorsal horns of the spinal cord, the substantia gelatinosa, that modulates the synaptic transmission of nerve impulses from peripheral (afferent) fibers to spinal cord transmission cells ( $T$ cells), and thus acts as a gate-control system. This gate mechanism is held to be influenced by the relative amount of activity in large diameter and small diameter afferent fibers, activity in the former tending to inhibit transmission and thus closing the gate, and activity in the latter tending to facilitate transmission and thus opening the gate. When the integrated firing level of the T cells reaches or exceeds a critical level, the firing is supposed to trigger a complex sequence of responses by the so-called action system, responsible for pain experience and response. The input of this system has access to neural systems involved in affective as well as sensory activities (Melzack \& Wall, 1965; Melzack \& Wall, 1982). In addition, several systems are proposed by which the brain may exert control over sensory input and spinal cord transmission. These influences from cognitive or higher central nervous system processes subserving attention, emotion, and memories of prior experience are thought to be mediated through the gatecontrol system. Furthermore, it is suggested that there are three psychological dimensions to the human pain experience, each with its own neurophysiological basis. The sensory-

\footnotetext{
S Specificity theory holds that pain, just like vision or hearing is a specific sensory modalty with its own netrophysiological structures. In patern theories, in particular the sensory interaction theory, pain is the result of intense stimulation of nonspecific receptors and stimulus intensity and central summetion of peripheral impulsc are critical determinants of pain. Also a specialized input-controlling system is assumed that normally prevents summation to take place. Destruction of this system leads to pathological pain states. In the concept of select of function sensory mechanisms have been identified by their particular function in pain processes (Mclzack \& Wall, 1965; Schmeider \& Karoly, 1983).
} 
discriminative dimension refers to the sensory qualities of pain in terms of temporal, spatial, pressure, thermal, and other properties of the sensation. The affective-motivational dimension refers to the affective qualities of pain in terms of tension, fear, and autonomic properties that motivate a person into action to alleviate the pain. Finally, the cognitive-evaluative dimension refers to thought processes involved in evaluating both the sensory and the affective components. This evaluation is mainly in terms of the meaning of the current situation, its relation to past experiences, and expected outcome of coping responses (Melzack \& Casey, 1968; Melzack \& Torgerson, 1971; Melzack \& Wall, 1982). It is assumed that these three dimensions interact with each other and influence motor mechanisms responsible for the complex pattern of observable behavior that characterizes pain. The foregoing then suggests that pain does not refer to a single sensation produced by a specific stimulus but instead can be considered "a complex perceptwal experience in which physiological and psychological/cogmitive processes interact, making pain a function of the whole individual, including his presen thoughts and fears as well as his hopes for the future" (Melzack \& Wall, 1982).

\section{The operant conditioning model}

The operant conditioning model as originally proposed by Fordyce (1976), stands in marked contrast with the biomedical view described earlier. It also departs from the gate-control model because the role of affective, cognitive, and to some extent sensory components of the pain experience is neglected (Turk \& Rudy, 1986). Although as such, it is a return to the earlier linear causal models of pain, it also meant a new era in thinking about pain.

According to Fordyce (1976), pain is not directly observable but can only be inferred from. the patients" communications, either verbal or non-verbal. The model proposes that when exposed to a stimulus that causes tissue damage, the immediate response of the individual is withdrawal or an attempt to escape from the noxious sensations. Activity believed to cause or exacerbate pain may be avoided, help might be sought to alleviate the pain and so forth. Furthermore, the individual might display several behaviors, verbal and non-verbal, which indicate that the person is in pain, such as moaning, crying, grimacing, posturing etc. Initially, in the acute phase, some of these activities and behaviors might automatically follow the noxious stimulus and may be viewed as respondents, controlled by antecedent stimuli. However, these observable behavioral manifestations are as such subject to the principles of learning and reinforcement and may develop unto chronic pain problems by means of operant conditioning principles. Pain behaviors may be maintained even in the absence of nociception by positive reinforcement, such as attention from a spouse or health care providers, financial compensation as well as by negative reinforcement such as the avoidance of undesirable or feared activities. Also, behaviors that foster recovery, such as activity or working might not be reinforced sufficiently (Fordyce, 1976; Turk, 1996a, 1996b; Turk \& Rudy, 1986, 1992). In a Jater study, Fordyce, Shelton, and Dundore (1982) show that in some instances pain behaviors persist solely because of avoidance learning. Furthermore, they hold that in these instances the pain behaviors not only persist because of anticipated negative social or situational consequences, but also because of anticipated internal nociceptive stimuli and consequent pain, thus leading to respondent conditioning. As time passes, the number of situations associated with pain may increase by means of stimulus generalization, thus contributing to even more avoidance. Anticipated distress rather than real distress (in the form of nociception or: exogenous reinforcement) is the key element in those cases.

\footnotetext{
"Closely in line with this is the so-called "fear-awoidance" model of chronic pain (Lethem, Slade, Troup \& Bentley, 1983) which holds that fear of pain can generate in some individuals a strategy of avoidance of physical and social activities rather than confrontation. leading to exaggenated pain perception and the subsequent physical and psychological reinforcement of the invalid status.
} 
In the operant formulation then, behavioral manfestations of pain (e.g, moaning, linping, avoidlance behaviors, complaining, grimacing) rather than pain itself are central and enphasis is placed on the communicative function of these behaviors which may cone under control of external contingencies of reinforcement (i.e., operant conditioning) (Turk, 1996a, 1996b) or internal reinforcement and avoidance leaming as in the case of anticipated distress (i.e., respondent conditioning) (Fordyce et al., 1982).

There are several studies supporting the role of operant and respondient aspects in chronio pain, although most of the evidence is correlational and some studies are hampered by methodological difficulties (Turk \& Flor, 1984). Of particular importance is a study by Flor, Kerns, and Turk (1987) who found that patients" pain reports were best predicted by spouse reinforcement, as perceived by the patient. Also, it was shown that patients' subjective activity level was best predicted by the punishing responses scales of the West Haven Yale Multidimensional Pain Inventory (WHYMPI) (Turk \& Rudy, 1988), indicating that patients with spouses who ignored their pain or responded negatively to it, showed higher levels of activity. In a similar study by Flor, Turk, and Rudy (1989), a positive relationship was shown between significant others' pain-reinforcing responses as perceived by the patients and the impact of their pain (WHYMPI severity and interference scales). However, this relationship was found to be mediated by sex, marital status, and marital statisfaction, and it was stronger for male patients who were married and weaker for patients who showed less marital satisfaction. In a more recent study (Romano, Tumer, Jensen, Friedman \& et al., 1995), using direct observational measures, it was found that spouse solicitous responses predicted physical dysfunction in patients with chronic musculoskeletal pain who were more depressed, and predicted the rate of non-verbal pain behaviors in those patients who reported higher pain severity. Although these data support the operant view on the role of spouses and significant others in maintaining pain and disability, they also indicate the importance of several qualifications relating to depression, pain severity, sex, and the marital rellation.

\section{The diathesis-stress model}

In 1.982, Flor was one of the first to propose a comprehensive diathesisustress model of chronic back pain in which physical, psychological, and social factors are integrated to explain the development of chronic back pain (unpublished manuscript, see Turk \& Flor, 1984). Although basically the same, the model was later refined and extended to the development of chronic pain in general and there was more emphasis on the role of learning processes (Flor \& Birbaumer, 1994; Flor et al., 1990).

The starting point in this psychobiological model of chronic pain is that pain behaviors are motivated by a combination of physiological and behavioral antecedents and consequences. More specifically, it is proposed that the development and maintenance of chronic pain results from the interaction of several components. At the core of the model is the concept of a "diathesis", that is a physiological predisposition involving a specific body system, e.g., the back muscles. This predisposition consists of a lowered threshold to noxious stimulation and results in the development of a physiological response stereotypy that is specific for an individual and is based on the diathesis. The response stereotypy most notably involves sympathetic activation and elevated muscle tension levels. Genetic factors, previous trauma, and social leaming experiences are all considered to be possible predisposing variables. The next component in the model consists of aversive internal or external stimuli (stressors) which may act as precipitating stimuli. These are pain-related or other stressors (e.g. familial conflicts or occupational pressures) with a negative meaning and personal relevance to the subject. The stressors are held to function as unconditioned and conditioned stimuli, activating the diathesis and motivating avoidance behaviors. A third component refers to inadequate or maladaptive behavioral, cognitive, or physiological responses to stressors c.g., 
increased perceived stressfulness of otherwise neutral stimuli, overinterpreting physical symptoms, increased and prolonged muscular reactivity, and sympathetic activation. The fourth and last component in the model concerns the rolle of learning processes as maintaining and reinforcing variables, more specifically, the role of operant and respondent (classical) conditioning. As mentioned before, the former involves pain behaviors being reinforced directly e.g., by attention of the spouse, indirectly by avoiding unpleasant activities, or the maintenance of pain behaviors by not reinforcing well behaviors. The latter involves the classical conditioning of fear of activity and subsequent avoidance of activity as well as the conditioning of increases in muscle tension and sympathetic activation. Thus a vicious paintension-avoidance circle might develop. It is lyypothesized that in most chronic pain syndromes these two learning processes are interacting.

In an extensive review article, discussing 60 studies on symptom specificity in chronic pain patients, Flor and Turk (1989) conchude that despite the numerous methodological problems, the data consistently suggest the presence of site-specific, stress-related psychophysiological responding in chronic back pain and headache patients, thus corroborating the diathesis-stress model. Methodologically more sound studies lending support to the diathesis-stress model are discussed in Flor and Birbaumer (1994). Finally, in a recent controlled study by Flor, Breitenstein, Birbaumer, and Furst (1995), chronic back pain patients with solicitous spouses were found to show reduced pain thresholds and pain tolerance levels when their spouse was present during a cold pressor test as compared to a spouse absent condition. Furthermore, the chronic back pain patients in this study demonstrated physiological response stereotypy in response to a personal significant stressor, inespective of the solicitousness of their spouse.

\section{The cognitive-behavioral perspective on chronic pain}

The term "cognitive-behavioral perspective" on chronic pain, as it is used in most research and treatments, refers to the way a person perceives, interprets and relates to his pain. The basic theoretical cognitive concepts are originally developed by, amongst others, Ellis, Beck, Meichenbaum, and Bandura (see e.g., Bandura, 1977; Beck, Emory \& Greenberg, 1985; Beck, Rush, Shaw \& Emory, 1979; Ellis, 1962; Meichenbaum, 1977, 1985). The term "cognition" comes from the Latin "cognoscere", meaning to apprehend. In psychology it is a generic term, not only used to refer to thoughts but also to attention, thinking, problemsolving, remembering, and other mental processes that are elements in the chain of information processing (Colman, 1994). Crucial in information processing is the formation of cognitive structures or cognitive schemata, a theoretical construct that can be conceptualized as a collection of knowledge on a particular concept. A particular schema is activated by a matching event and is thought to have a controlling function on behavior and the processing of information, thereby determining what is perceived -i.e., attention-, how information is interpreted, and which actions are taken (Arntz, 1991). In cognitive psychology it is hypothesized that in some cases, stch as in depression, obsessive-compulsive disorder, or panic disorder, but also in non-clinical cases like racial prejudices, certain schemata have become hyper accessible and have come to dominate the information processing. That is, schema congruent information is held to be selectively prioritized for further cognitive processing. Such sehema congruent information processing may, in case of maladaptive schemata, lead to distortions in the interpretation of information (i.e, negative automatic thoughts) and to excessive emotions and behaviors (Arntz, 1991). In this respect the presence of pain may change the way an individual processes pain-related and other information (Turk., $1996 \mathrm{a})$.

From the cognitive-behavioral perspective then, people with chronic or recurrent pain have negative expectations about their ability to perform specific activities, believe that they have limited ability to exert any control over their pain, and more generally adopt a negative set 
about pain and how it will affect their lives (Flor et al., 1987; Turk \& Fernandez, 1991). These maladaptive appraisals about the situation in turn are thought to have direct effect on physiology by increasing autonomic and sympathetic nervous system arousal and potentially, muscle spasm, as well as an indirect effect by reducing physical activity, leading to reduced muscle flexibility, strength, and tone. Also, with modern neural maging techicues relationships between cognitive processes (such as attention, learning, expectancy) and central brain processes have been found in studies using experimentally induced pain (see e.g., Bantick, Wise, Ploghaus, Clare, Smith \& Tracey, 2002; Ploghaus, Narain, Beckman, Clare, Bantick, Wise, Matthews, Rawlins \& Tracey, 2001; Ploghaus, Tracey, Clare, Gati, Rawlins \& Matthews, 2000). Furthermore, these negative cognitive interpretations are hypothesized to reinforce the experience of demoralization and dysphoric mood, symptom preoccupation, inactivity, and overreaction to nociceptive stimulation which will influence behavioral. response. Finally, they may also affect how people will present symptoms (overt pain behaviors) to significant others, thereby evoking responses that may reinforce the pain. behaviors and impressions of the seriousness, severity, and uncontrollability of the pain (Tunk \& Fernandez, 1991; Turk \& Rudy, 1992; Weisenberg, 1994).

To summarize, in the cognitive-behavioral perspective on chronic pain, people are considered active processors of information, and behavior and enotions are thought to be strongly influenced by interpretations of events rather than solely by the characteristics of the events themselves. Also, cognitions, emotions, behaviors, and the social environment are held to be causally related to some extent. This means that cognitions may elicit and influence emotions and behavioral responses which conversely may influence environmental responses and facilitate or inhibit particular cognitions, a process referred to as reciprocal determinism (Turk, 1996b; Turk \& Fernandez, 1991; Turk \& Rudy, 1992).

Support for the important role of cognitive factors in attention to pain and pain-related variables, such as pain intensity and disability, comes from a number of studies of which a few will be mentioned here. Crombez, Eccleston, Bayens, and Eelen (1998a) found a larger attentional task performance disruption immediately after the onset of a low intensity pain stimulus in subjects receiving threat instructions as compared to a control group not receiving threat instructions. They further hypothesized that this threat interference is strongly related to the urge to escape from pain or avoid pain, thus prioritizing the object of threat for attention. This process might also be influenced by individual differences in attentional priorities, such as the presence of catastrophic thinking about pain. Indeed, Crombez, Eccleston, Bayens, and Eelen (1998b) demonstrated that students who were classified as highly catastrophizing on pain, exhibited a more pronounced task interference, i.e., attentional disruption, immediately after the onset of somatic threatening information (expecting a highly painful stimulus or being told that pain fibers were stimulated). Eccleston and Crombez (1999) propose a cognitive-affective model of pain in which the emphasis is on the interruptive and attention demanding function of pain. They state that there are several factors that moderate the interruption by pain. Amongst these factors are the threat of pain, the novelty of the pain stimulus, distraction, the predictability of a pain stimulus, as well as emotional arousal. They also hold that the facilitating effect of threat of pain on the emergence of pain is greater in people who interpret pain-related stimuli as threatening and as highly likely to have catastrophic consequences. Flor and Turk (1988) found that situational pain-related cognitions (pain-related coping and pain catastrophizing self-statements such as, "I should relax" or "this pain is driving me crazy") as well as more general cognitions concerning control over pain (e.g., "I have no control over my pain") were highly related to reports of pain severity and disability in a sample of rheumatoid arthritis patients as well as in a heterogeneous sample of chronic back pain patients. Both sets of cognitive variables in combination explained between 32 and $60 \%$ of the variance in pain and disability respectively. Furthermore, the proportion of 
Variance explained by disease-related variables, such as pain duration or amount of degenerative change on spinal $\mathrm{X}$ rays, did not add significantly to the proportion of variance atready explained by the cognitive variables. More studies supporting a cognitive-behavional perspective are discussed in Turk (1996a, 1996b), Turk and Rudy (1992), Turk and Rudy (1986), and Turk et al. (1983).

The provisional conclusion then from the available research is that cognitive variables play an important role in (chronic) pain and are significantly associated with pain-related variables, such as pain intensity and disability. This conclusion holds, despite the fact that most of the studies on cogntive variables in (chronic) pain are correlational and many of them are fraught with methodological problems. The overall impression is that the cognitive-behavional perspective on chromic pain is a valid one, worth to be explored in further research.

In the next paragraph some shortcomings and limitations of the aforementioned models will be discussed.

\section{Shortcomings and limitations of the aforementioned models}

The fact that the models discussed previously all have made significant contributions to the understanding of (chronic) pain and have their merits is beyond doubt. Nevertheless, they have some shortcomings and limitations that will be dealt with below. From this discussion the choice of the main subject of this thesis will be explained.

Although some of the physiological details of the gate-control theory of pain have been challenged (Nathan, 1976), no one has yet proposed an alternative theory or has refuted successfully its basic tenets. Research findings however suggest that these basic tenets are not so much incorrect as they are too general. Also, the interactions in the important anatomic structures involved in the model are far more complex than the model suggests and there is a greater diversity of endogenous pain-modulating systems than suggested by the theory (Price, 1988). The gate-control theory of pain recognizes the importance of cognitive-evaluative factors in the experience of chronic pain but the model is rather static and does not explain how cognitive factors combine or interact with sensory and affective factors to define the pain experience. Furthermore, the theory holds that cognitive processes only modulate pain-related emotional states while currently it is accepted that there is a causal link between cognitive processes and the production of affect (Price, 1988). Finally, the gate-control theory does not incorporate the role of reinforcennent and learning factors.

The operant conditioning model on the other hand does this of course, but it neglects the role of cognitive factors altogether by focusing exclusively on pain behaviors (e.g., grimacing, moaning, limping, posturing). The presence of pain is inferred from these behaviors which poses the problem that certain behaviors (e.g., limping) might actually be the result of an underlying physical abnomality rather than being associated with the process of pain. Moreover, any behavior can be subsumed under the heading "pain behavior" which makes the "pain-behavior" construct over-inclusive and lacking specificity (Turk, 1996b). Also, heavy emplasis is placed on the communicative function of these behaviors. This tholds the risk that sone of these alleged pain behaviors might be considered as inappropriate or maladaptive, although strictly speaking the operant conditioning model is neutral in this respect.

At the core of the diathesis-stress model are the concepts of diathesis and response stereotypy as well as the importance of the personal relevance of a stressor. These concepts do seem to have some significance, at least for chronic back pain patients and headache patients. However, the link between psychophysiological measures and pain is still unclear at present. furthermore, one of the assumptions of the diathesis-stress model, that chronic pain patients should also demonstrate a prolonged return to baseline levels in relevant psychophysiological measures after stress exposure, is not corroborated unequivocally by research findings (Flor \& Turk, 1989). More problematic is the fact that in the later and broader formulation of the 
diathesis-stress model, the model is extended to include a whole array of variables, annong which leaming factors, coping factors, cognitive variables, and fear variables, all interacting with each other to produce the pain experience and pain reaction (sed Flor \& Bitbamer. 1994; Flor et al., 1990). Using this model it is difficult, if not impossible to generate and test specifte predictions, leaving this model virtually insensitive to falsification which is a prerequiste for a scientifically sound theory.

The same critical remarks may apply to the general formulation of the cognitive-behavioral perspective on chronic pain, in which the operant conditioning emphasis on extemal remforcement and the corresponding vew of learned fear and avoidance (respondent conditioning) is integrated within the framework of information processing in which attention, thinking, problem solving, remembering, and other mental processes are all considered important elements. A consequence of this is that treatments based on the cognitive-behavoral model often inchude a complex mixture of ingredients (e.g., exposure, problem-solving training, relaxation training, cognitive techniques) which makes it difficult to determine the specific effective ingredients and to compare the results of different treatument studies. Knowing which specific treatment elements are effective for what kind of chronic pain patients is important to be able to customize treatments. Therefore, a good theory or model ("good" in the scientific sense, meaning parsimonious and able to generate specific and testable predictions is indispensable. This finally brings us to the choice of the main subject of this thesis which is one of the core elements of the cognitive-behavional model of pain: pain catastrophizing. By focusing on a specific aspect of the cognitive-bethavioral modet, it should be possible to formulate more specific and better testable hypotheses. Before formulating the research questions of the current thesis, it is necessary to discuss in more detail the notion of "catastrophizing". 
CHAPTER 3

Pain catastrophizing

"Pleasure is off a visitant but pain clings crwelly to us" John Keats 


\section{Pain catastrophizing}

In the following sections the concept of pain catastrophizing will be discussed into more detail. After defining pain catastrophizing and placing it within the context of cognitive theory, some conceptual and methodological issues will be addressed which should help to clearly outline the concept of pain catastrophizing. Research findings will be presented, demonstrating the important role of pain catastrophizing in relation to pain-related variables such as pain intensity and disability, and a model of how pain catastrophizing might be related. to these variables is introduced. Finally, the outline of this thesis will be discussed.

\section{Orientation and definition}

As mentioned previously, in the cognitive-behavioral perspective on chronic pain, emphasis is placed on the interpretation of the painful experience rather than on the painful experience itself. It is hypothesized that the presence of pain may activate particular schemata, prioritizing schema congruent information for further cognitive processing, thereby changing the way a person will process pain-related and other information. This in turn may lead to distorted and negative interpretation of such information, excessive and negative emotions and behavior. These negatively distorted interpretations or belliefs about oneself or one's situation are referred to as cognitive errors (Turk, 1.996b). Turk (1996b) refers to several publications in which cognitive errors and distortions are consistently linked to depression, subjectively reported pain intensity, and disability. One of these cognitive errors is catastrophizing. The concept of catastrophizing goes back quite a long time and was used over four decades ago by Albert E1lis (1962), one of the founding fathers of cognitive-behavioral therapy. He incorporates the concept of catastrophizing in one of a number of ubiquitous irrational ideas which would lead to widespread neurosis: "the idea that it is awful and catastrophic when things are not the way one would very much like them to be" (Ellis, 1962). According to Ellis (1962) a rational human being should "perceive his own tendency to catastrophize about inevitable unfortunate situations to tell himself: 'Oh, my Lord" How tertible this situation is VT positively cannot stand it!'- and should question and challenge this catastrophizing ${ }^{\text {r. }}$ Later, Beck et al. (1985; 1979) described catastrophizing as a thinking error or cognitive error that involves dwelling on "the worst possible outcome of any situation in which there is a possibility for an unpleasant outcome". He stated that "the anxious person overemphasizes the probability of this catastrophic outcome and uswally exaggerates the possible consequences of its occwrence" (Beck et al., 1985).

Although not defined explicitly or uniformly, the concept of catastrophizing also emerged in the realm of pain research, in particular research conceming the possible effects of cognitive factors and strategies on the experience of pain. In an experimental cold pressor study; Spanos, Radke Bodorik. Ferguson, and Jones (1979) conceived catastrophizing as worrying and having anxious thoughts about pain imagining negative consequences of pain. and thinking about other more severe situations, or focussing on and exaggerating the unpleasantness of the painful situation (e.g., "I kept thinking I can"t stand this much longer, I want to get out"). Rosenstiel and Keefe (1983) developed a questionnaire for assessing cognitive and behavioral pain coping strategies, the Coping Strategies Questionnaire (CSQ). One of the seven coping strategies included in the questionnaire is catastrophizing which involves negative self-statements, catastrophizing thoughts and ideation (e.g." "I worry all the time whether it will end" or "it"s terrible and it's never going to get any better"), reflecting a sense of helplessness in dealing with the pain experience. Chaves and Brown (1987) conceptualized catastrophizing as engaging in cognitive activity which exaggerates the fearful aspects of a painful situation. This may involve negative self-statements, catastrophizing thoughts, and catastrophizing imagery. 
However, these studies differentially emphasize the content of these negative pain-related cognitions which reflects elements of exaggeration or magnification, worrying or rumination, and helplessness or pessimism with respect to the ability to deal with the pain (Sullivan, Thorn, Haythomthwaite, Keefe, Martin, Bradley \& Lefebvre, 2001b). Sullivan, Bishop, and Pivik (1995) sought to answer the question whether or not the different elements from which the concept of catastrophizing is construed of, reflect different dimensions of catastrophizing. They developed a self-report measure of catastrophizing, the Pain Catastrophizing Scale (PCS), that incorporated the elemerts of rumination (see Spanos et al., 1979), helplessness (see Rosenstiel \& Keefe, 1983), and magnification (see Chaves \& Brown, 1987). The results of a principal componenis analysis demonstrated that the elements of rumination. helplessncess, and magnification constitute three separate, although related factors or dimensions of pain catastrophizing.

The conclusion from the foregoing is that there are no explicit defining criteria for the construct of catastrophizing and that catastrophizing appears to be made up of at least three separate although related dimensions: helplessness, rumination, and magnification (Sullivan et al., 1995). Based on these considerations and research findings, the following definition of pain catastrophizing is proposed here: a negative ortentation to pain itself or its possible consequences which is expressed in rumination about pain, cognitions reflecting a subjectively experienced inability to deal with pain, and cognitions reflecting a tendency to magnify or exaggerate possible outcomes of pain. This definition will be adhered to in the current thesis. People who catastrophize about their pain may keep thinking about their pain, consider themselves unable to deal with it, and have extremely negative cognitions about the pain itself or its possible consequences. This may be expressed in cognitions such as, "I keep thinking how much it hurts", "there is nothing I can do to reduce the intensity of the pain", and "I wonder if something serious might happen" (see Sullivan et al., 1995).

Before elaborating on the research findings concerning the role of pain catastrophizing in (chronic) pain, it is important to spend a few words on some conceptual problems regarding the construct of catastrophizing.

\section{Conceptual issues}

Viewing the pain literature, it is striking that the concept of pain catastrophizing is not used unequivocally: some consider it to be a separate construct and a distinct cognitive variable, others hold it to be confounded with anxiety, depression, or negative affectivity. Some refer to it as a form of adjustment to pain, others refer to it as a coping strategy, as inadequate or maladaptive coping, or even as the absence of adequate coping, while still others refer to pain catastrophizing as negative beliefs or appraisals with regard to pain. This of course has consequences for the way the concept of pain catastrophizing is operationalized and measured, making a direct comparison between studies in which different conceptualizations of catastrophizing are employed rather difficult. In the following sections these issues will be addressed.

\section{Catastrophizing: appraisal and belief, or coping?"}

Undoubtedly, one of the most robust findings in contemporary psychological pain research is the important role of pain catastrophizing. Despite the growing body of research on pain catastrophizing (for an extensive overview, see Sullivan et al., 2001b), there is still conceptual confusion about the construct, which revolves around the question whether or not pain catastrophizing is to be considered a form of coping. An illustrative example of the polemic

\footnotetext{
"This paragagh is a shghty adopted version of the wanuseript wat is published as: Severeijms, R... Vhayen, J.W.S., yan den Hout, M.A. (2004). Do we need a communal coping model of pain catustrophizing? An altenative enplanation. Pan. $1 / 1 / 3 j, 226-229$
} 
around this question can be found in a 1999 issue of Pain Forum (Geisser, Robinson \& III, 1999; Haythornthwaite \& Heinberg, 1999; Keefe, Lefebvre \& Smith, 1999; Thorn, Rich \& Boothby, 1999). Related to this conceptual confusion is the lack of a guiding theoretical framework (Keefe, Rumble, Scipio, Giordano \& Perri, 2004). Sulliwan et al. (2001b) give an extensive and a thorough review of the literature on pain catastrophizing and discuss several models that can have a heuristic function and may help to sort and understand the research data on pain catastrophizing. One of these models in particular, the communal coping model of catastrophizing (CCM) (Sullivan et al., 2001b; Sullivan, Tripp \& Santor, 2000b) is clearly taking root in pain research lately.

In this section a case is made for placing pain catastrophizing within the transactional stress and coping model of Lazarus and Folkman (1984). It is argued that the CCM in its current formulation might actually contribute to the conceptual confision around the construct of pain catastrophizing that was mentioned before. This finally leads to the question of whether we actually need a CCM of catastrophizing.

In their transactional model of stress and coping Lazarus and Folkman (1984) make a clear distinction between the concepts of beliefs, appraisal, and coping. With the concept of beliefs Lazarus and Folkman (1984) refer to a person characteristic that is an important determinant of appraisal. Particularly important for the current discussion are generalized beliefs about personal control that have to do with feelings of mastery and confidence. They are conceptualized as stable personality dispositions. According to Lazarus and Folkman (1984) appraisal can be understood as an evaluative process. They distinguish between primary appraisals that involve evaluating a particular event as irrellevant, benign-positive, or stressful with regard to a person's wellbeing and secondary appraisals that involve evaluating a particular event with respect to coping options and their possible effectiveness. Both interact with each other and influence whether and which coping efforts will be attempted. Finally, coping is defined as both behavioral and cognitive efforts to manage specific external and/or internal demands that are appraised as taxing or exceeding the resources of the person (Lazarus \& Folkman, 1984).

In 1995 Sullivan et al. (1995) already discussed that at descriptive level there are similarities between the three subscales of the Pain Catastrophizing Scale (PCS) on the one hand (i.e., magnification, rumination, and helplessness) and primary and secondary appraisal processes on the other hand. Magnification and rumination may reflect a focus on and evaluation of painful stimuli as extremely threatening, whereas helplessness reflects the evaluation of painful stimuli as unable to cope with. Furthermore, people may possess enduring beliefs or schema about the threat value of painful stimuli or their ability to effectively cope with painful stimuli (Sullivan et al., 1995).

There are some research findings that support these similarities, e.g., the lhigh test-retest: reliability of the PCS, the close association between pain catastrophizing and other appraisall constructs, and the lack of association between pain catastrophizing and forms of coping (for a more detailed description see Sulliwan et al., 1995; Sullivan et al., 2001b). Furthermore. associations have been found between pain catastrophizing and trait constructs, such as trait anxiety (Sullivan et al., 1995) and neuroticism or negative affectivity (Affleck, Temen, Urrows \& Higgins, 1992; Drossman, Leserman, Li, Keefe, Hu \& Toomey, 2000; Sullivan et al., 1995). In Figure I we propose an appraisal model of pain catastrophizing, based on the transactional model of stress and coping (Lazarus \& Folkman, 1984). 
Figure 1.

Proposed appraisal model of pain callastrophizing

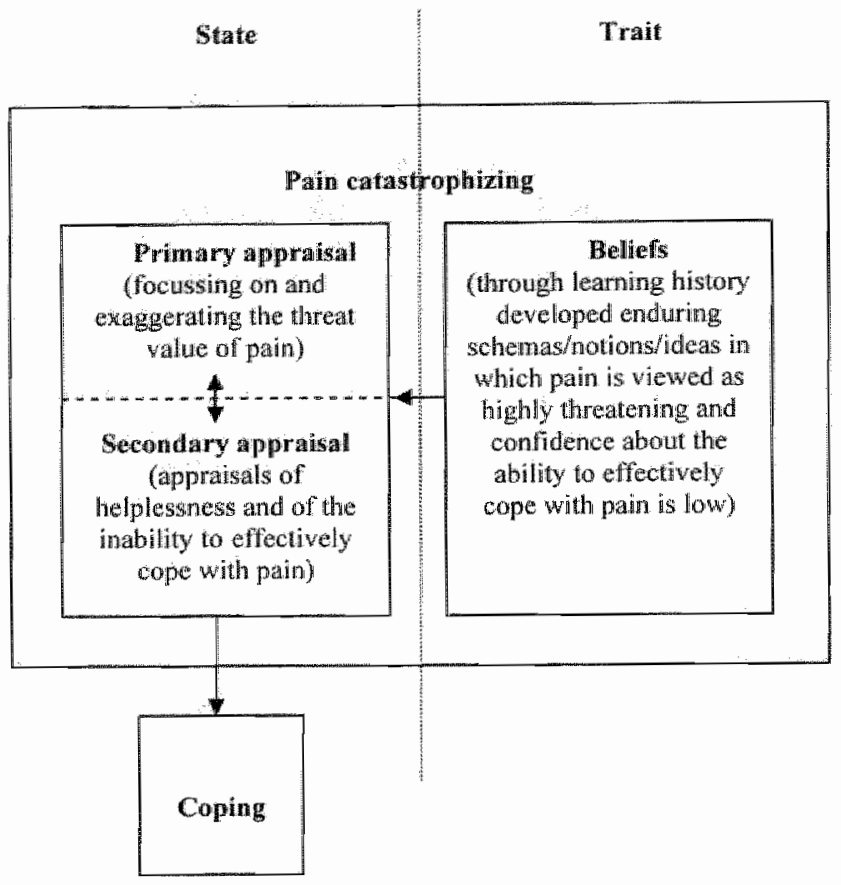

Based on the finding that pain catastrophizing mediates the sex-pain relationship, Sullivan et al. (2000b) and Keefe, Lefebvre, Egert, Affleck, Sullivan, and Caldwell (2000) proposed a communal coping model of pain catastrophizing. In this model, pain catastrophizing is conceptualized as being part of a broader, interpersonal or communal coping style in which it serves a social communicative function. At the core of the model is the tenet that dealing with stress within a social, interpersonal, or relational context, for instance, by soliciting social support, assistance, or empathic reactions from (significant) others, might be more important than pain or stress reduction per se. To the extent that catastrophizing serves this goal it may be considered adaptive although it has detrimental consequences for the pain problem itself.

However, placing pain catastrophizing within a communal coping framework pisses over the fact that pain catastrophizing is a theoretical construct with an explicitly cognitive connotation. Therefore, it is rather confusing to operationalize catastrophizing other than in a cognitive way. Furthemore, these catastrophizing cognitions or appraisals can not serve a social communicative function in and of themselves because they are not observable. Rather, their sheer existence can only be inferred by asking people to complete the PCS or the CSQ or from the behaviot, etther verbal ot nonverbal, of those who catastrophize about pain. This behavior, of course, may or may not serve a social communicative function. So, pain catastrophizing could only serve a social communicative function indirectly. Again, however, it is quite confusing to define the nature of a process, which is cognitive in the case of pain catastrophizing, in terms of its potential function or one of its effects. Analogously, although one of the effects of pain behavior might be that people become less active and develop a disuse syndrome, this does not mean that pain behavior should be defined in terms of disuse. 
Therefore, we argue here that the term "pain catastrophizing" should be used in its pure, that is, cognitive meaning. By placing catastrophizing within a coping framework, the conceptual confusion that already exists may even be enhanced.

According to Sullivan et al. (2001b) several research findings support a CCM explanation of catastrophizing. For example, Keefe, kashikar-Zuck, Robinson, Salley, Beaupre, Caldwell, Baucom, and Haythornthwaite (1997) found that catastrophizing was related to lower spousal ratings of self-efficacy for pain. More recently, some hypotheses, derived from the CCM have been examined in research. Keefe, Lipkus, Lefebvre, Hurwitz, Clipp, Smith, and Potter (2003) found that cancer patients who catastrophized about their pain reported receiving higher levels of caregiver instrumental support. Caregivers of these patients reported having higher levels of stress and critical behaviors and rated the patients as having more pain and engaging in more pain behaviors. Giardino, Jensen, Turner, Ehde, and Cardenas (2003) showed that there was a positive assoctation between pain catastrophizing on the one hand and solicitousness and pain reports on the other hand. Perceived solicilousness and the type of social relationship (spouse or partner versus someone else) moderated the association between catastrophizing and pain reports. Finally, Sullivan, Adans, Sullivan, and Willians (2004) found that compared to low catastrophizers, high catastrophizers displayed pain behaviors for a longer duration and used fewer pain coping strategies in the presence of an observer.

Overall, the results of these studies seem to support a CCM interpretation of pain catastrophizing in that they, at least partially, confirm some of the predictions derived fiom the CCM, and demonstrate that pain catastrophizing has interpersonal correlates. Nevertheless, the question is justified whether the same predictions can also be derived from existing models of pain catastrophizing, in particular the appraisal model of pain catastrophizing for which supporting research data have been found as well.

According to the appraisal model of pain catastrophizing, primary appraisal processes (magnification and rumination) and secondary appraisal processes (helplessness) interact and determine whether and which coping efforts will be attempted. Indeed, several studies that have started from the transactional model of stress and coping have found specific relationships between appraisals of stressful events and ways of coping (Dunkel-Schetter, Feinstein, Taylor \& Falke, 1992; Dunkel-Schetter, Folkman \& Lazarus, 1987; Folkman, Lazarus, Dunkel-Schetter, DeLongis \& Gruen, 1986; Knussen \& Lee, 1998; Turner, Clancy \& Vitaliano, 1987). More specifically, researchers have found that threats to one"s own physical health were associated with more seeking of social support (Folkman et al., 1986). Also, events that were appraised as highly stressful were characterized by support from significantly more people and by greater amounts of informational and emotional support than were events that were appraised as low in stress (Dunkel-Schetter et al., 1987). Finally, perceived stressfulness of having cancer was associated with significantly greater coping through social support (Dunkel-Schetter et al., 1992).

The point to be made here is that from this perspective and based on these findings, predictions may be made that individuals who focus on their pain (rumination), think that something terrible might happen to them because of the pain (magnification), and feel unable to effectively cope with their pain (helplessness), may adopt a coping style that, either intentionally or not, elicits social support and attention by means of overtly displaying distress, fear, helplessness, or pain behavior. The prediction of the CCM that the presence of other people serves as a discriminative stimulus for these overt displays of distress is not surprising in this context, for what is the use of showing, for example, that you'te helpless if there is no one around to respond or to help you?

In a recent study by Sullivan et al. (2004) in which supportive evidence for the CCM is reported, it is proposed in the discussion section that "strategies used to maximize the proximity of others may be motivated, at least in part, by low levels of coping efficacy" (italics 
by the present author). However, in our opinion they fail wo follow this hypothesis to its logical and ultimate consequence, which is that exactly for that reason it may be unnecessary to hypothesize that some individuals prefer a more communal or interpersonal approach to coping and that cuatastrophizing plays an important role in communicating this approach. Rather, within an appraisal model of catastrophizing, it is the features of catastrophizing itself that directly impinge on these interpersonal correlates. In this view, the predictions derived from the CCM of pain catastrophizing can also be accounted for by the appraisal model of pain caltastrophizing.

In summarizing the previous discussion, some tentative conclusions and recommendations can be made. First, an appraisal model of pain catastrophizing offers an attractive theoretical framework, is supported by research data, and might function as a guide for future catastrophizing research. Second, pain catastrophizing should be defined in terms of its cognitive nature and not in terms of its potential function or effects. Third, the fact that catastrophizing has interpersonal correlates is intrinsic to the very nature of pain catastrophizing and can be attributed to the effects of appraisals of threat and helplessness (that is, low levels of coping efficacy) on coping behavior (that is, overt display of distress and helplessness, as well as pain behavior), which in turn is likely to evoke social attention and support. Finally, to the extent that these conclusions can be validated empirically, we doubt that there is a need for a CCM of catastrophizing in which it is hypothesized that individuals who catastrophize about their pain do so because they prefer an interpersonal approach to dealing with painful stimuli. Instead, we would rather argue the opposite, namely that these individuals seek assurance and social support because they focus on their pain, experience their pain as threatening, and feel helpless in dealing with their pain. Of course, further research will have to demonstrate which of these two models, the CCM or the appraisal model, has more merits.

\section{Is pain catastrophizing confounded with depression, negative affectivity, and anxiery?}

Another already briefly mentioned and potentially serious problem is the possible conceptual overlap between pain catastrophizing and depression, negative affectivity, and anxiety. On theoretical grounds it might be more obvious to expect a relationship between pain catastrophizing and fear/anxiety than between pain catastrophizing and depression because pain catastrophizing refers to perceived or possible (future) threat, to which the usual reaction. is fear. Nevertheless, Beck et al. (1979) proposed a cognitive model of depression in which cognitions and beliefs are of crucial importance in the production and mantenance of depresisive symptomatology. He suggests that depressed individuals can be characterized by faulty information processing reflected in cognitive errors -among which catastrophizing-, leading them to construe the world, themselves, and their experiences in a negative way.

Indeed, specific cognitive errors and distortions, including pain catastrophizing, have been consistently linked to depression (see Turk, 1996b). Furthermore, in a study addressing the question of whether pain catastrophizing is to be considered a separate cognitive variable or merely a component of depression, Sullivan and D'Eon (1990) found that the relationship between coping with pain (as measured with the CSQ) and depression was primarily accounted for by the relationship between pain catastrophizing and depression. In addition, all of the items of the pain catastrophizing subscale of the CSQ were judged by six clinical. psychologists as somewhat or very reflective of depressive symptomatology (Sullivan \& D'Eon, 1990). It was concluded that pain catastrophizing might rather be a cognitive manifestation of depression than reflecting distinct aspects of pain-related cognitions. Also, in a study using path analysis to examine causal relationships between neuroticism, pain coping, pain catastrophizing (as measuied with the CSQ), chronic mood, and chronic pain (Affleck et al., 1992), the path linking pain catastrophizing to chronic pain failed to reach significance 
when depression was controlled for. This suggests that the relationship between pain catastrophizing and chronic pain is accounted for by depression. In this respect, two studies are worth mentioning, both using the CSQ to measure pain catastrophizing. In the first study, using path analysis, Geisser, Robinson, Keefe, and Weiner (1994b) found that pain catastrophizing mediates the relationship between depression and the affective and evaluative aspects of pain. In the second study, Keefe, Brown, Wallston, and Caldwell (1989) examined the role of pain catastrophizing in a sample of rheumatoid arthritis patients on two occastons, separated by 6 months. Pain catastrophizing at time 1 was found to be positively correlated with depression. However, more interesting is the finding that in addition to depression at time 1, pain catastrophizing at time 1 was the only significant predictor of depression at time 2 , even when controlling for the significant autoregressive effects of the depression variable. Although a correlational design was used, the results do suggest that pain catastrophizing has unique explanatory power over and above its relation with depression. Evidence that pain catastrophizing is a separate construct from depression comes from other studies as well (see e.g., Geisser, Robinson \& Henson, 1994a; Hassett, Cone, Patella \& Sigal, 2000; Keefe et al., 2000; Sullivan, Stanish, Waite, Sullivan \& Tripp, 1998).

Similarly, high correlations between pain catastrophizing and measures of neuroticism/negative affectivity and anxiety have been found, which may lead one to assume that there is considerable conceptual overlap between both constructs. In an experimental study, employing a cold pressor task, the relationship between pain catastrophizing, as measured with the Pain Catastrophizing Scale (PCS), and measures of depression, trait anxiety, negative affect, and fear of pain, as well as the ability of these variables to predict pain responses was examined (Sullivan et al., 1995). The results of this study showed that pain catastrophizing was significantly correlated with depression, trait anxiety, negative affect, and fear of pain. However, only pain catastrophizing and fear of pain were significantly correlated with pain during the ice water immetsion. Moreover, only pain catastrophizing contributed unique variance to the prediction of pain when controlling for all the other variables. Other studies as well showed that although correlated will neuroticism/negative affectivity, pain catastrophizing has incremental value as a predictor of several pain-related outcome variables (Affleck et al., 1992; Drossman et al., 2000; Martin, Bradley, Alexander \& Alarcon, 1996).

In summarizing the discussion on the conceptual confounding of pain catastrophizing with basic emotional constructs such as depression, negative affectivity/neuroticism, and anxiety, it is important to realize that the mere fact that pain catastrophizing is related to these variables does not necessarily imply that there is considerable conceptual overlap or confounding. Moneover, theoretically it is to be expected that there is considerable metrical confounding. For if the theory is correct that pain catastrophizing can promote, amongst others, fear/anxiety and depression, significant correlations between the measures of pain catastrophizing, fear/anxiety, and depression can be expected as well. Nevertheless, apart from these more theoretical remarks, it does seem justified to conclude that, although pain catastrophizing is related to depression, negative affectivity, and lear/anxiety, the concept of pain catastrophizing appears to have unique explanatory power, thereby illustrating its potential. use fulness in the study of (chronic) pain.

\section{Pain carastrophizing and (chronic) pain: research findings and theoretical framework}

Research findings suggest that it might be more important for adequate coping with pain to avoid pain catastrophizing than to actively engage in adequate coping strategies and that the presence of pain catastrophizing is crucial in poor coping with pain, rather than differences in adaptive coping strategies (Chaves \& Brown, 1987; Heyneman, Fremouw, Gano, Kirkland \& Heiden, 1990; Kenda 11, 1992; Newton \& Barbaree, 1987; Rosenstiel \& Keefe, 1983; Tumer 
\& Clancy, 1986). Reviewing the literature, Jensen, Turner, Romano, and Karoly (1991) hold that despite several methodological shortcomings of the studies reviewed, some consistent conclusions can be drawn regarding the role of pain catastrophizing in (chronic) pain. There appears to be a clear relationship between pain catastrophzing on the one hand and psychological and physical dysfunction on the other hand. Also, pain catastrophizing appears to predict long-term adjustment to chronic pain and may partially explain the relationship between pain severity and adjustment. In more recent years, evidence has been accumulating from cross-sectional, prospective, as well as from experimental studies, that pain catastrophizing is significantly associated with various pain-related outcome variables across both clinical and non-clinical populations and across various pain conditions.

In Table 2 an overview of these studies is given. It should be kept in mind that the studies presented in Table 2 used different measures of pain catastrophizing, used different control variables in the analyses, and used different pain subgroups as well. The majority of the studies are cross-sectional and overall demonstrate a significant association between pain catastrophizing and pain intensity, pain-related disability, depression, psychological distress, anxiety, and negative affectivity, across different pain subgroups. Prospective studies are smaller in number and are more diverse with respect to the specific pain samples and interventions used. This makes it more difficult to draw general conclusions. Nevertheless, the results suggest that for people with various kinds of (chronic) musculoskeletal pain there is a significant prospective association between pain catastrophizing and disability, depression, and pain intensity, although there are also studies that failed to find significant prospective results. The few experimental studies are too specific to draw general conclusions.

Exactly how pain catastrophizing might be related to pain intensity, pain-related disability, and psychological distress is described in a cognitive-behavioral model (Vlaeyen, Kole Snijders, Boeren \& van Eek, 1995a; Vlaeyen, Kole Snijders, Rotteweel \& Ruesink, 1995b). In this modell it is hypothesized that if pain, possibly caused by an injury, is catastrophized, this will lead to pain-related fear, more specifically fear of movement or (re)injury which in turn will promote avoidance behavior, followed by disability, disuse, and depression. The latter will maintain the pain experiences, and thus reinforce the vicious circle of increasing fearavoidance. There are several studies corroborating this model and suggesting that pain catastrophizing is a precursor of pain-related fear (Crombez et al., 1998b; Crombez, Vlaeyen, Heuts \& Lysens, 1999; Sullivan et al., 1995; Vlaeyen et al., 1995a; Vlaeyen et al., 1995b). This model will be used as the theoretical framework from which the research in the current study is started on. The model is summarized in figure 2.

Figurie 2 .

The "fogit" "avoudane nodel

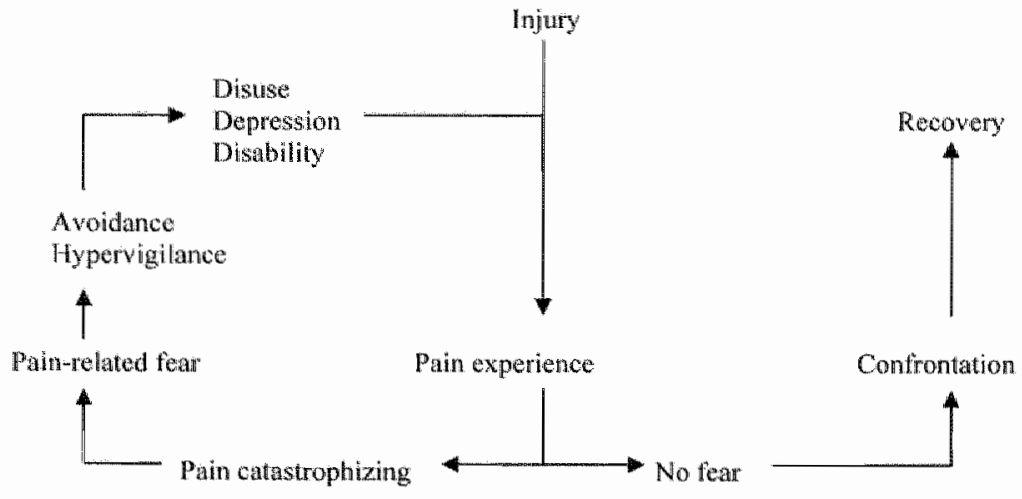




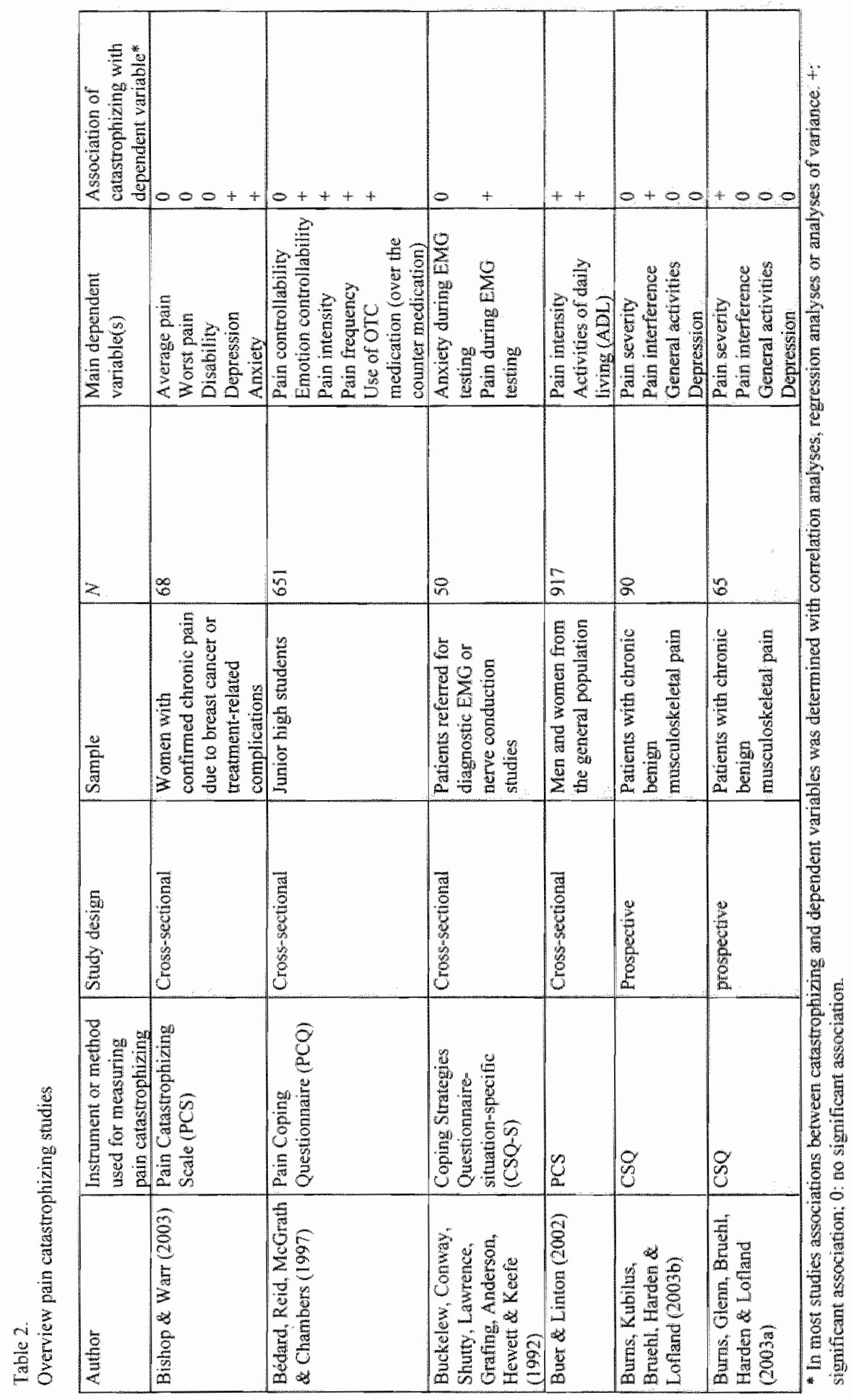




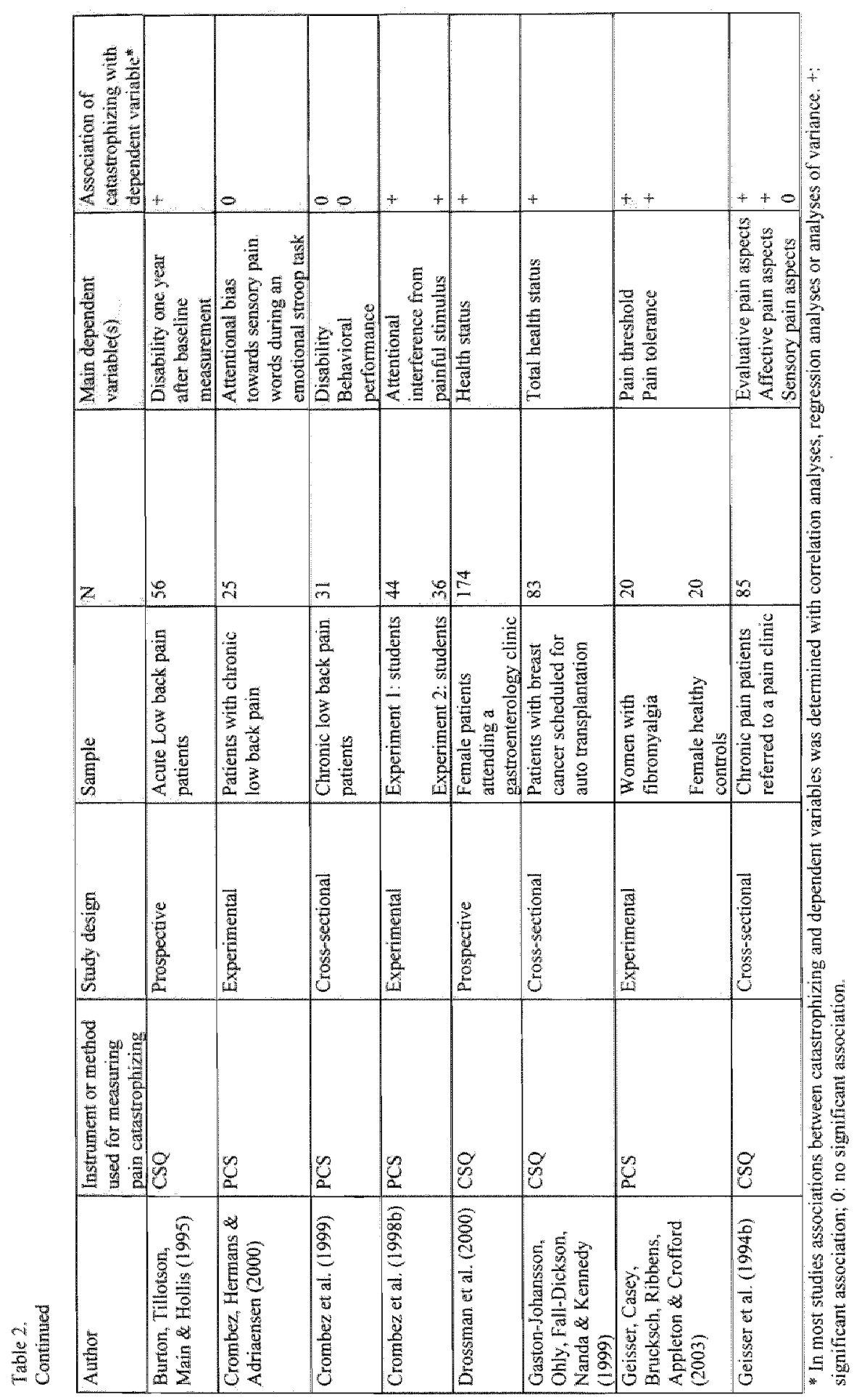




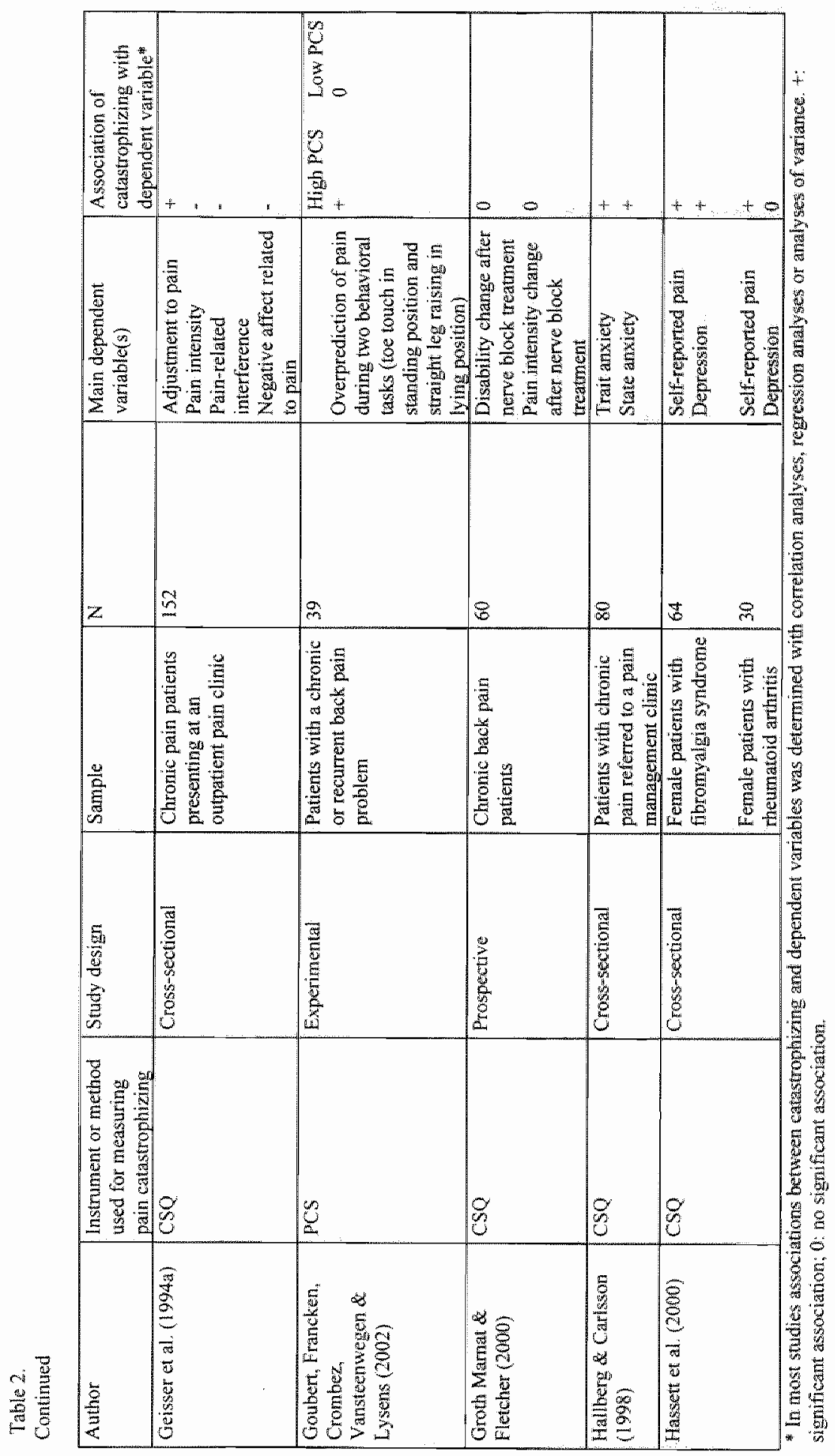




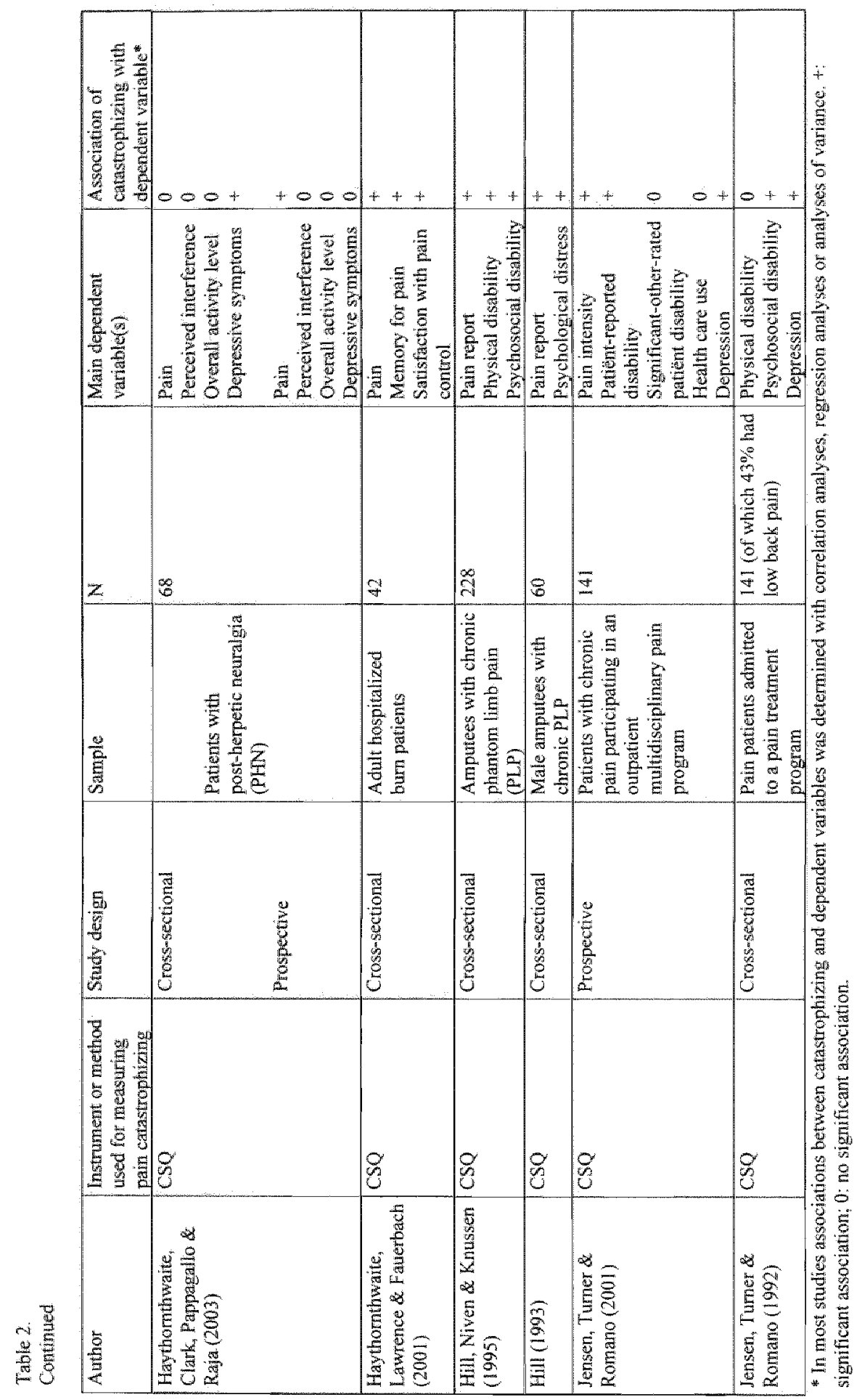




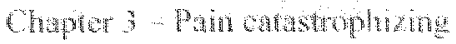

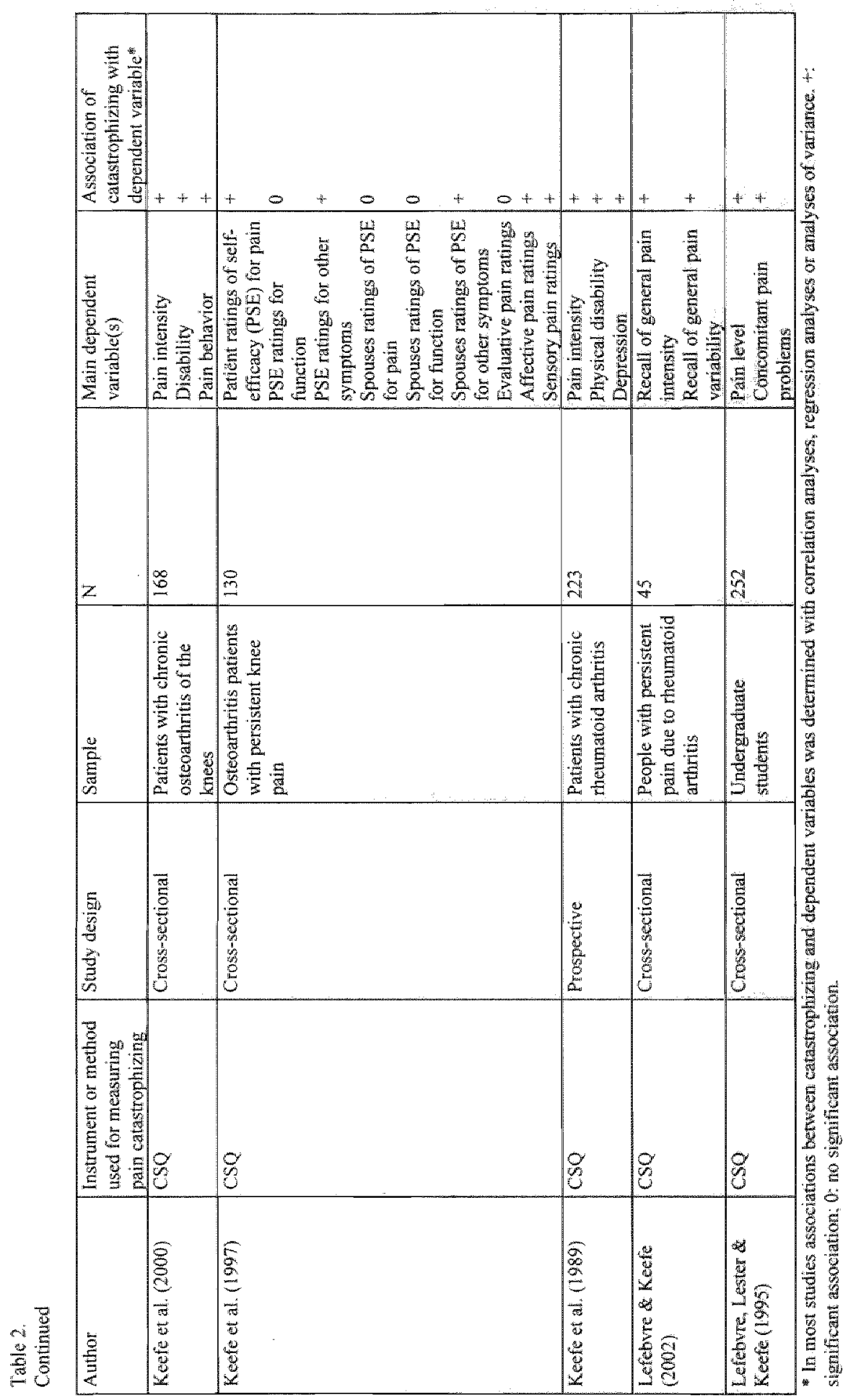




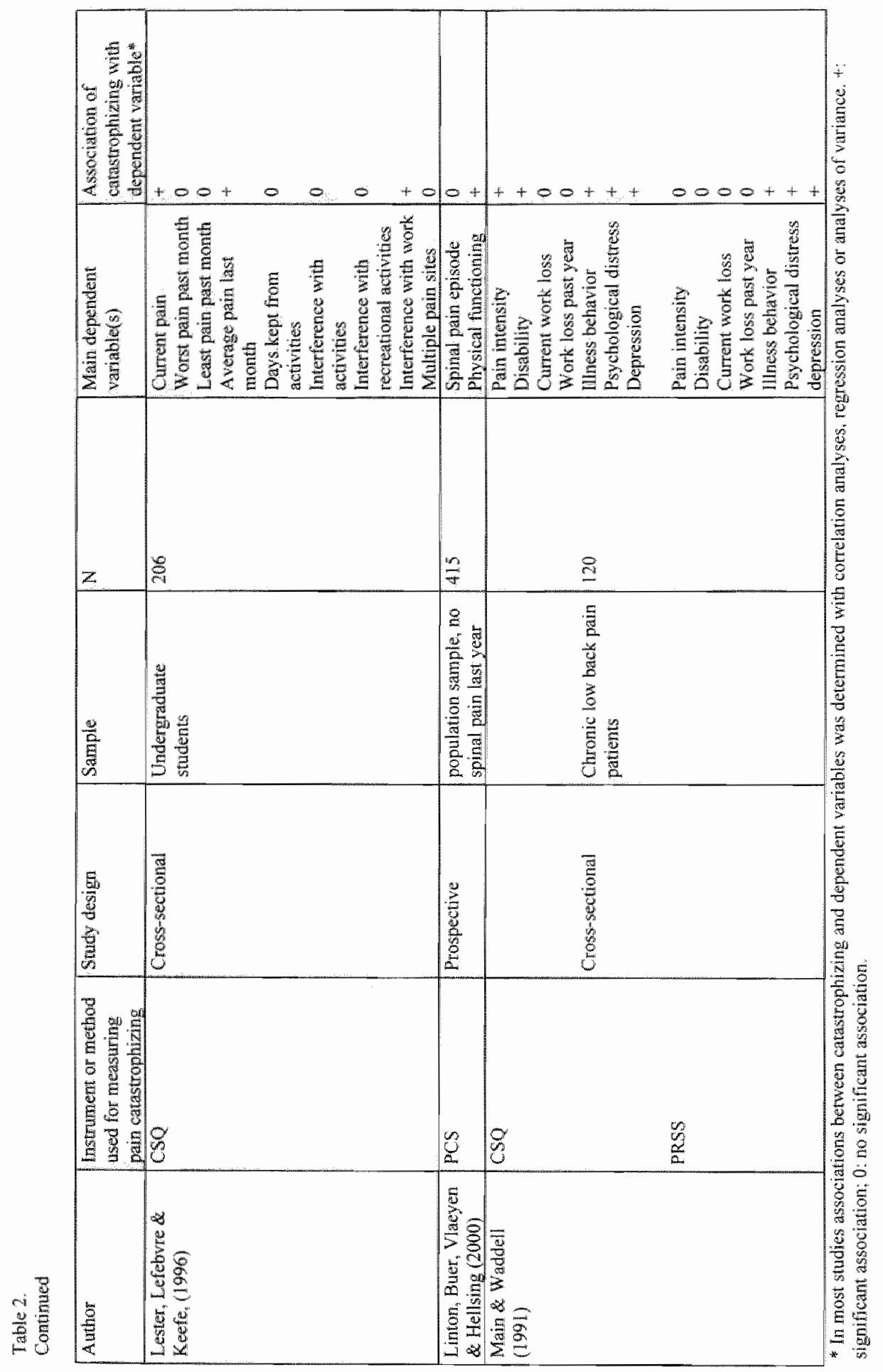




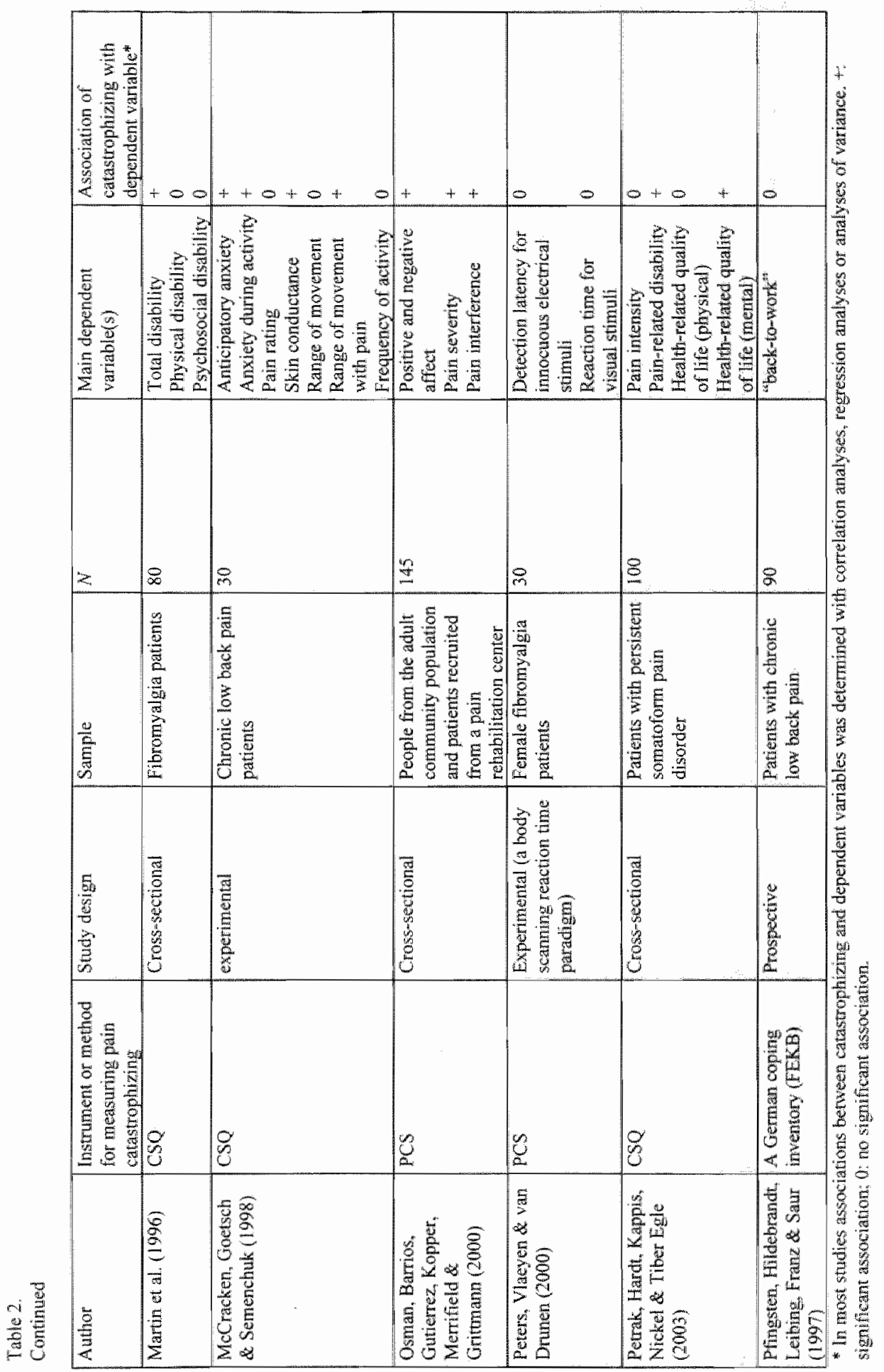




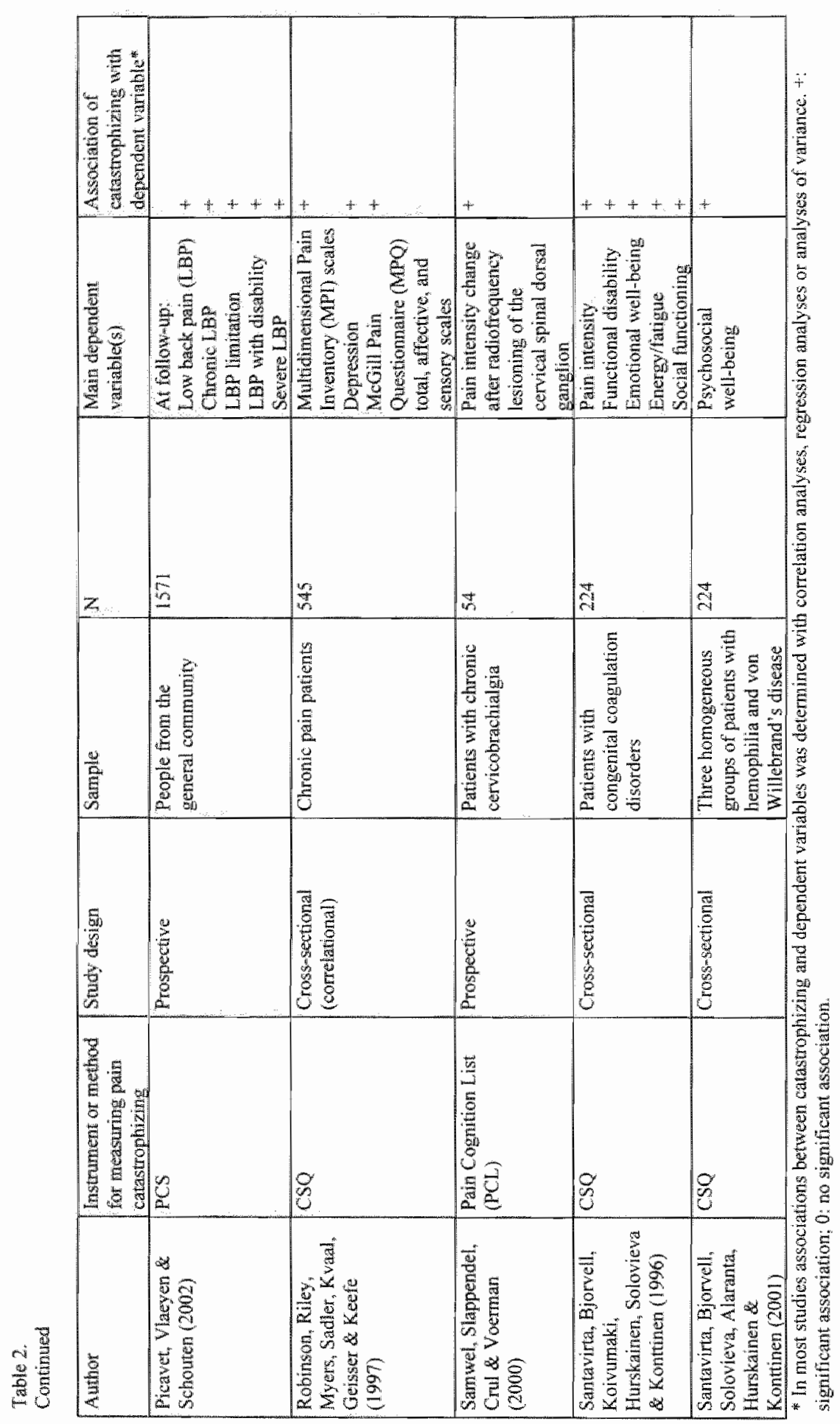




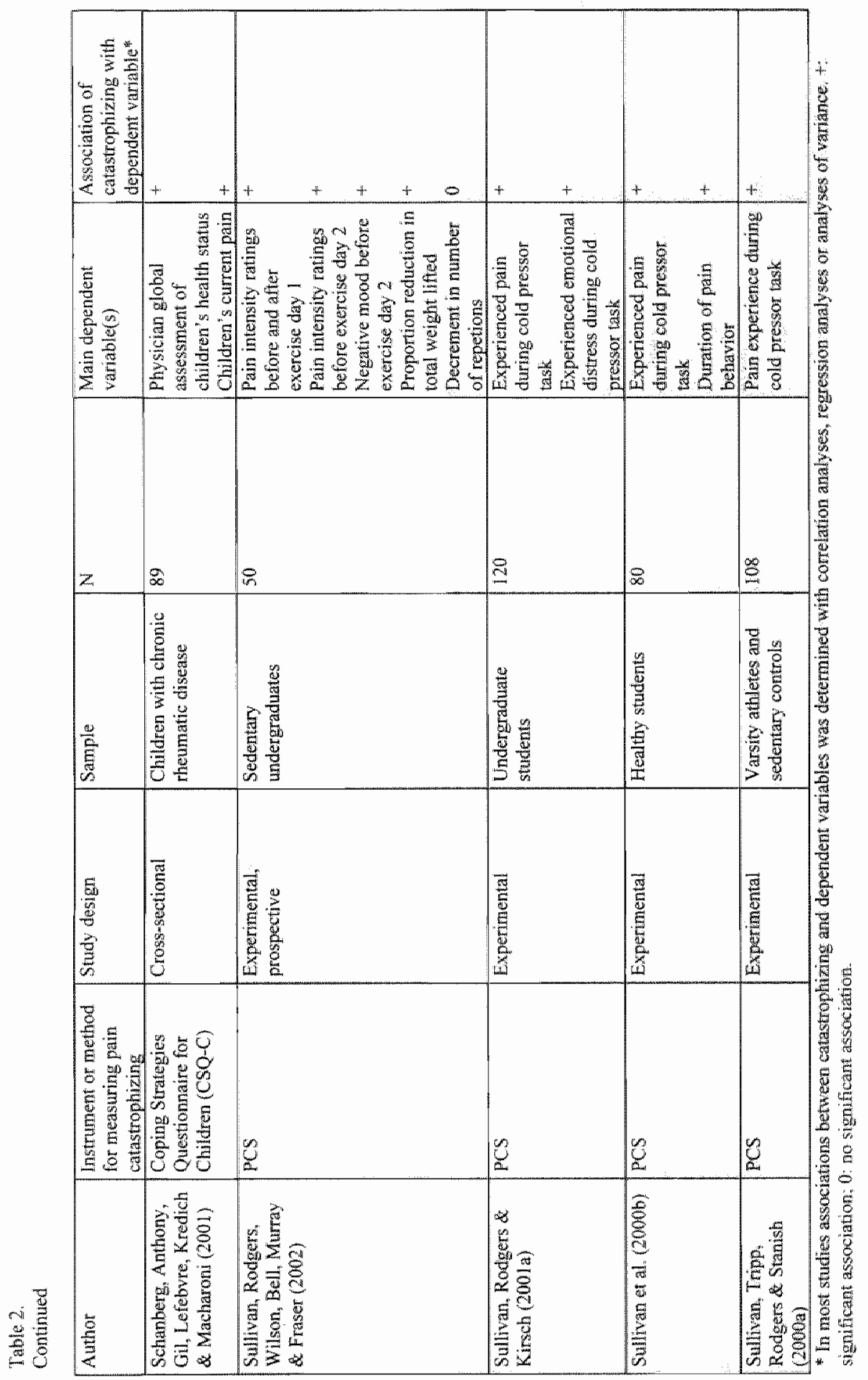




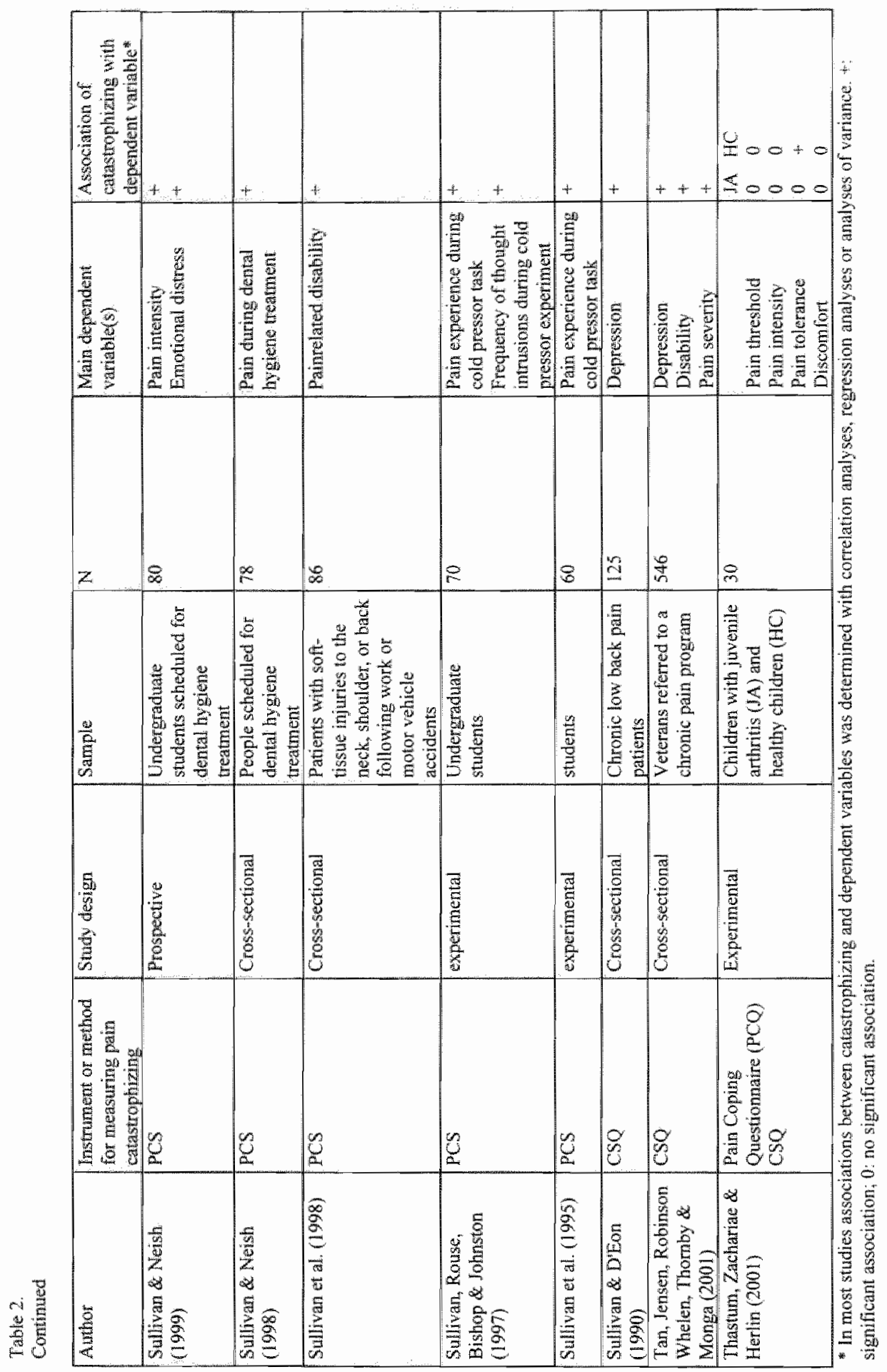




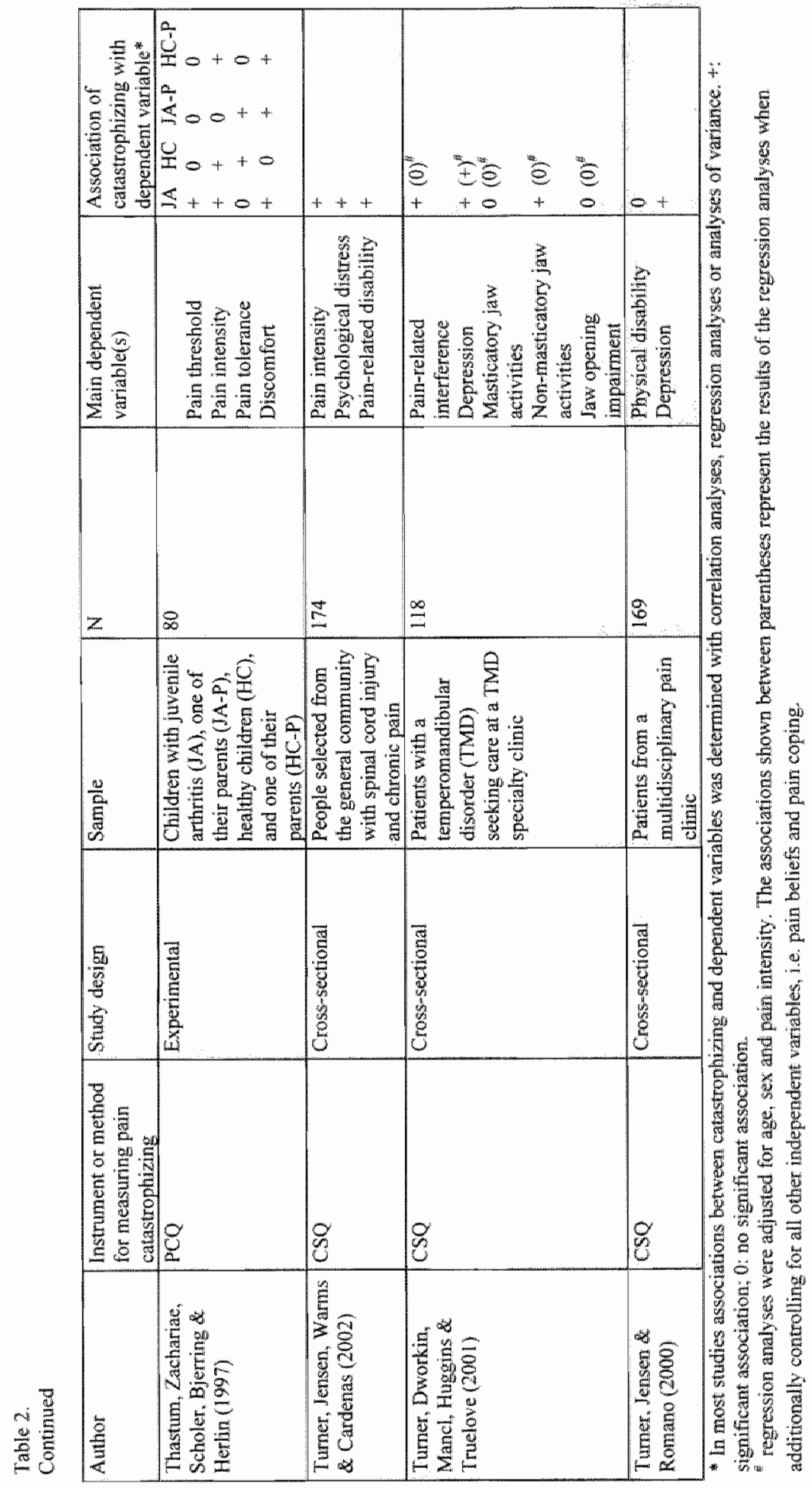




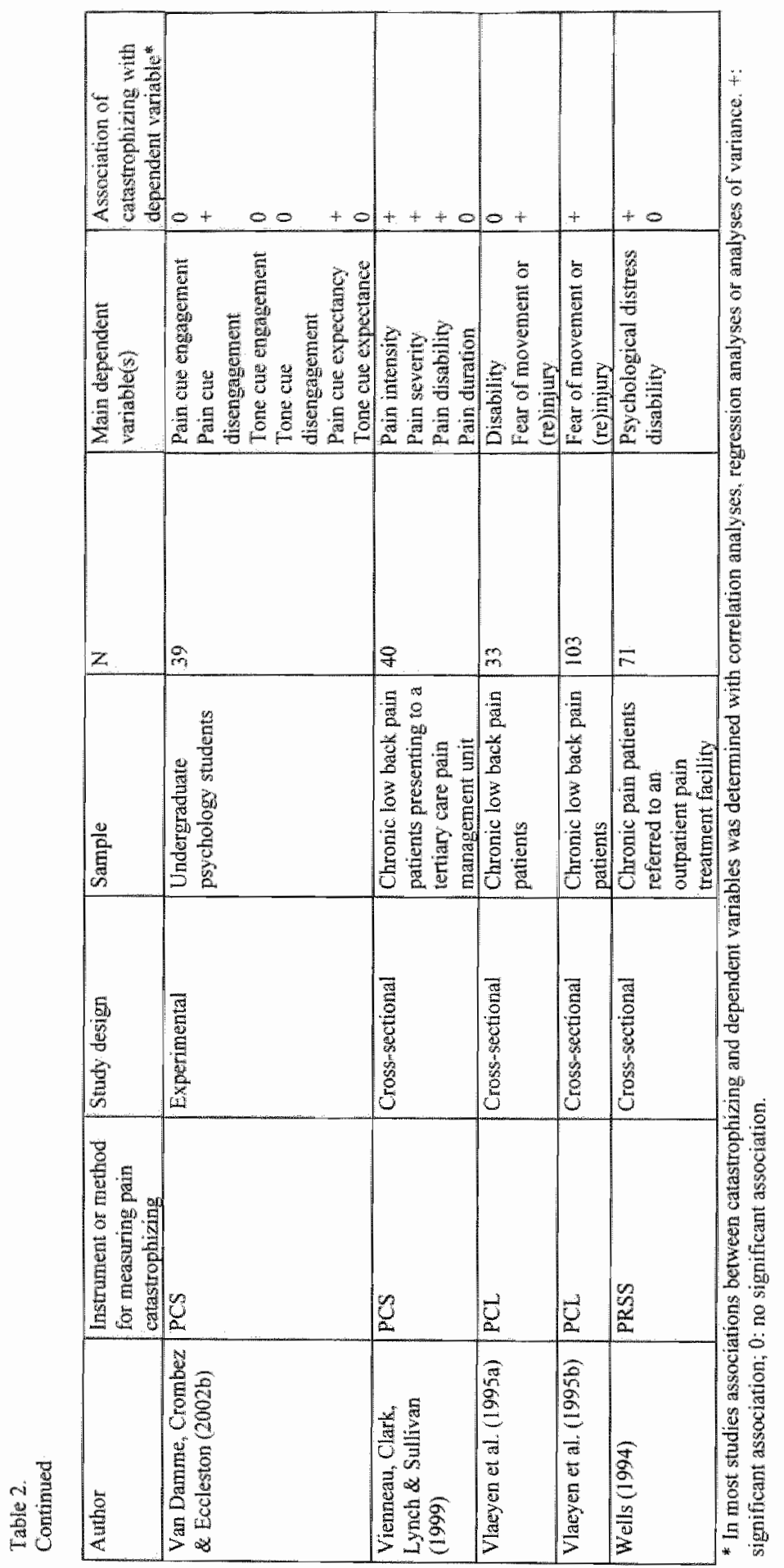




\section{Ontline of this thesis}

As highlighted in the previous sections, pain catastrophizing, conceptualized as a set of negative beliefs and appraisals about pain, appears to be a vallid construct that has unique explanatory power over and above basic emotional constructs such as anxiety, depression, and negative affectivity and is consistently found to be related to pain intensity, disability, psychological distress and other pain-related outcome variables. Nevertheless, some questions still remain to be answered. These questions will be addressed in the current thesis which includes one clinical, cross-sectional study, three general population studies (two cross sectional studies and one prospective study), two experimental studies, and a general discussion of the results.

1. Is carastrophizing about pain related to pain intensity, pain-related disability, and psychological distress, independent of the level of plysical impaimem? Are these associations the same for different groups of patients with chronic pain?

Alhough only moderate associations between objective physical impaiments on the one hand and pain and disability on the other hand have been found, it is still possible that the level of physical impaiment confounds the association between pain catastrophizing and pain intensity, disability and psychological distress. Indeed, there are at least two studies that suggest a significant relationship between pain catastrophizing and physical impairment (Main \& Waddell, 1991; Reesor \& Craig, 1988). In this respect, pain catustrophizing may simply be an epiphenomenon of physical impairment. We are not aware of any studies that examined whether pain catastrophizing is related to pain-related outcome variables independent of the level of physical impaiment. Therefore, chapter 4 describes the outcomes of a study in which the associations between pain catastrophizing and pain intensity, disability, and psychological distress are examined, adjusting for the level of physical impairment.

For exploratory reasons, it is also examined whether these associations are the same for different subgroups of patients with chronic pain. Significant associations between pain catastrophizing and pain-related outcome variables have been found across a variety of different pain problems, but there are no studies that directly compare different pain subgroups. Although the fear-avoidance model (Vlaeyen et al., 1995a; Vlaeyen et al., 1995b) does not predict such differences, there is a preliminary idea that does predict these differences to occur. This idea is that different types of pain may differ with regard to their respective threat value. For example, back pain and hip pain might be considered as intrinsically more threatening and incapacitating because they involve larger regions of the body, involve larger muscle and bone contributions, or contain vital nerve tracks that serve important functions such as locomotion (spinal cord), and therefore might lead to greater incapacity and interference with activities. Based on this idea, it can be hypothesized that differences in threat value among different pain subcategories would cause the specilic pain subtype to moderate the associations between pain catastrophizing and the health indices examined in the current study. This interaction effect between pain catastrophizing and pain subtype is tested as well in this study.

2. Is catastrophizing about pain related to general health status in the general community? Can the three-factor structure of the Pain Catastrophizing Scale (PCS) be replicated in the general population and is it invariant across different subgrowps of musculaskeleial pain? Are there indications for the reliability and validity of the PCS in the general population? 
Significant associations between catastrophizing about pain and both physical and mental health indices have been found in both chinical and non-clinical studies. However, this leaves some issues unresolved which are addressed in chapter 5 . In clinical samples relatively high base rates of physical and mental pathology can be expected which may have biased previous results. Also, the non-clinical samples often consist of pain-free subjects such as students which may well limit the generalizability of the results to other populations, such as nonclinical pain-suffering subjects. Replicating prewiously found results conceming the relationship between pain catastrophizing and general health status in a large community sample would therefore yield valuable supportive infonmation about the role of pain catastrophizing in (chronic) pain. Furthermore, it would help to further validate, and refine models of risk factors for pain-related dysfunction such as the fear-avoidance model (Vlaeyen et al., 1995a; Vlaeyen et al., 1995b) discussed previously.

Similarly, the psychometric properties of the PCS - a widely used instrument to measure catastrophizing about pain- have only been evaluated in clinical and non-clinical populations but not in a community sample yet. It would be useful to know whether the same psychometric qualities of the PCS apply in community samples as in clinical or non-clinical samples.

3. Do people in the general community who catastrophize about thetr pain hove a higher incidence of specialist consultation for their pain complaints, of using pain medication, and of absenteeism because of their pain complaints or being disabled from work because of their pain?

There is growing evidence that catastrophizing about pain fosters the maintenance of chronic pain problems and thus may contribute to the detrimental consequences of chronic pain in terms of health-care utilization (e.g.s specialist consultation and use of medication) and loss of productivity (e.g., due to absenteeism and work related disability). Associations between pain catastrophizing and several health indices have been reported in various sellected patient samples. This may have biased the results because relatively high base rates of both physical and mental pathology are to be expected in patient samples, resulting in a high level of healthcare utilization and loss of productivity. Confirming these associations in a large Dutch community sample would greatly support the extemal validity of earlier findings. This issue is addressed in chapter 6 .

4. Do people in the general community with musculoskeletal pain who carastrophize about their pain have a higher risk of developing chronic pain complaints over a six months period than people who do not catastrophize about their pain? Do these people also have a higher risk of consulting a specialist for their complaints, of using pain medication, and of absenteetsm becouse of their complaints? Are any of these relationships moderated by the mumber of pain complaints or by pain intensity?

Little is known about the possible role of catastrophizing in the development of chronic complaints and of the prospective relationship between pain catastrophizing and several pain outcomes in the greneral population. This is an important void because as mentioned previously, in clinical samples relative high base rates of both physical and mental pathology can be expected and the frequent use of pain-free participants in non-clinical samples limits the generalizability of the results to other populations such as non-clinical pain-suffering subjects. This question is examined in chapter 7. Identifying pain catastrophizing as a risk factor for the development of chronic musculoskeletal pain would be supportive of the fearavoidance model discussed previously and would make it possible to further specify and 
identify the people at risk for developing chronic musculoskeletal pain complaints rather easily by screening them with the PCS. Elaborating on the previous research question, it will also be examined whether catastrophizing about pain prospectively affects specialist consultation, the use of pain medication, and absenteeism. Because there are indications that pain intensity and the number of pain complaints are also important in explaining several outcome variables it will be examined whether they function as moderator variables in the relationships mentioned above.

5. Does making people (temporarily) carastrophize about pain during a cold pressor task increase the level of expected pain and moximum experienced pain, and does it result in a shorter duration of the ice-wates immersion?"

6. Does (temporamily) reducing the level of catastrophizing about pain in patients with (chronic) recurrent low back pain improve rask performance and does if reduce expected pain and maximum experienced pain during a knee-exrension-flexton task?

The fear-avoidance model (Vlaeyen et al., $1995 \mathrm{a}$; Vlaeyen et al $1995 \mathrm{~b}$ ) predicts that pain catastrophizing promotes avoidance/escape behavior and the development of (chronic) pain. Several studlies have been performed that corroborated the fear-avoidance model and the important role of pain catastrophizing in (chronic) pain. However, most of these studies are cross-sectional and do not allow for any judgment about the causal role of pain catastrophizing. There are some prospective studies that suggest that pain catastrophizing is a precursor of pain rather than a consequence. Nevertheless, these prospective studies do not give a definite answer about the causal role of pain catastrophizing either. This important issue is addressed in two experimental studies. in which the level of catastrophizing is manipulated and the effects of this manipulation on task performance and pain are examined. In chapter 8 the results of a study with healthy controls is described in which an attempt is made to make people catastrophize about pain during a cold pressor task. Chapter 9 discusses the results of a study with people with chronic recurrent low back pain in which an attempt is made to reduce catastrophizing about back pain.

In chapter 10 a summary and discussion of the main findings of this thesis is presented as well as their implications, both theoretical and clinical. Also, the strengths and limitations of the studies presented in this thesis are discussed. 


\section{CHAPTER 4}

Pain catastrophizing predicts pain intensity, disability, and psychological distress independent of the level of physical impairment

This chapter is a slightly adapted version of the article that is published as: Severeijns, R., Vlacyen, J.W.S., Hout, wan den, M.A., \& Weber, W.I.J. (2001). Pain catastrophizing predicts pain intensity, disability, and psychological distress independent of the level of physical impairment. The Clinical Joumal of Pain, 17, 165-172. 


\begin{abstract}
The aim of the current study was to examine the cross-sectional associations between catastrophizing and pain intensity, pain-related disability, and psychological distress in a group of patients with chronic pain, controlling for the level of physical impairment. Furthermore, it was examined whether these associations are the same for three subgroups of chronic pain patients: those with chronic low back pain, those with chronic musculoskeletal pain other than low back pain, and those with miscellaneous chronic pain complaints, low back pain and musculoskeletal pain excluded.

Participants in this study were 211 consecutive referrals presenting to a university hospital pain management and research center, all of whom had a chronic pain problem.

Overall, chronic pain patients who catastrophized about pain reported more pain intensity, felt more disabled by their pain problem and experienced more psychological distress. Regression analyses revealed that catastrophizing was a potent predictor of pain intensity, disability, and psychological distress, even when controlled for physical impairment. No fundamental differences between the three subgroups were found in this respect. Finally, it was demonstrated that there was no relationship between physical impairment and catastrophizing.

It was concluded that for different subgroups of chronic pain patients, catastrophizing plays a crucial role in the chronic pain experience, significantly contributing to the variance of pain intensity, pain-related disability, and psychological distress. These assaciations are not confounded by the level of physical impairment. Some clinical implications of the results are discussed. Finally, it was concluded that these results support the validity of a cognitivebehavioral conceptualization of chronic pain-related disability.
\end{abstract}




\section{Introduction}

Recently, there has been a growing focus on the role of psychological factors in cluronic pain research. From this research, some consistent findings are emerging. First, in chronic pain, pathophysiological processes do not adequately explain the levels of pain and disability that chronic pain patients report (Feuerstein et al., 1987; Vlaeyen et al., 1995b; Waddell, 1987; Waddell \& Main, 1984; Waddell, Newton, Henderson \& Somerville, 1993; Waddell, Pillowsky \& Bond, 1989). In other words, there is no strong relationship between objective physical impairment ${ }^{7}$ and pain and disability. Second, catastrophizing, which is defined as an extremely and exaggeratedly negative orientation to pain itself or its possible consequences, appears to play an important role in the chronic pain experience (Geert Crombez, Baeyens, Vervaet \& Vlaeyen, 1997; Geert Crombez, Vervaet, Lysens, Baeyens \& Eelen, 1998c; Geert Crombez et al., 1999; Main \& Waddel1, 1991; Reesor \& Craig, 1988; Vlaeyen et al., 1995a; Vlaeyen, Seelen, Peters, de Jong, Aretz, Beisiegel \& Weber, 1999) and has been consistently linked to, amongst others, pain intensity, pain-lelated disability, and psychological distress (Flor, Behle \& Birbaumer, 1993; Flor \& Turk, 1988 ; Geisser et al., 1994b; Hill, 1993; Hill et al., 1995; Keefe et al., 1989; Main \& Waddell, 1991; Martin et al.., 1996; Sullivan \& D'Fon, 1990; Sullivan et al., 1998; Wells, 1994). Third, there are some prospective studies that suggest that catastrophizing is a precursor of pain problems rather than a consequence (Burton et al., 1995; Klenerman, Slade, Stanley, Pennie, Reilly, Atchison, Troup \& Rose, 1995; Linton et al., 2000; Linton \& Hallden, 1998). These findings corroborate a cognitivebehavioral conceptualization of chronic pain, elaborated in a model by Vlaeyen et al. (1995b), which assumes that catastrophizing in relation to pain promotes fear of movement or (re)injury. The latter in turn, leads to avoidance behavior, disuse, disability, and depression.

Nevertheless, some questions remain to be resolved. Although there appears to be only a moderate relationship between physical impairment and pain intensity and disability, there are at least two studies that suggest a significant relationship between catastrophizing and physical impairment (Main \& Waddell, 1991; Reesor \& Craig, 1988). Main and Waddell (1991) found a significant relationship $(r=.32, p<.001)$ between objective physical impairment and catastrophizing, as measured by the Coping Strategies Questionnaire (CSQ) (Rosenstiel \& Keefe, 1983) subscale. Reesor and Craig (1988) found that patients with chronic low back pain (CLBP) who displayed more non-organic signs and symptoms ${ }^{8}$ (termed "incongruent" patients) had greater physical impairment and disability and catastrophized nore about their pain than so-called "congruent" CLBP patients. In line with this, it can not be ruled out that physical impairment is a confounding variable, however remote that possibility might be. In contrast, if the cognitive-behavioral model of catastrophizing and fear of movement or (re)injury (Vlaeyen et al. 1995b) is valid, it is to be expected that the relationship between catastrophizing and pain intensity, pain-related disability, and psychological distress holds, irrespective of the level of physical impaiment. "This has yet to be demonstrated in research.

The aim then of the current study was to investigate the relationship between catastrophizing and pain intensity, pain-related disability, and psychological distress in a

\footnotetext{
${ }^{6}$ Disability is defined as any restriction or lack of ability to perform an activity in the manner or within the range considered normal for a human being that results from an imparment (World Health Organization defintion).

"Physical impaiment is defined as "an anatomical or pathological abnombalty leading to loss of normal bady abillity".

Non-organic signs atre defined as "behaviors elicited daring an orthopedic examination proedure which deviate from anatonical principles", non exaggenated and do not conform to anatomy or disease course".
} 
group of patients with chronic pain. We hypothesized that overall, chronic pain patients who catastrophize, experience more pain intensity, feel more disabled by their pain problem, and experience more psychological distress. To rule out the possibility that physical impaiment is a confounding variable, it will be analyzed as a covariate. For exploratory reasons, we will also examine whether these relationships apply to different subgroups of chronic pain patients (i.e. whether there is an interaction effeet between catastrophizing and group membership). Confirming the aforementioned hypotheses across homogeneous subgroups with different medical diagnoses would signify additional support for the important role of catastrophizing in the chronic pain experience. The following subgroups are distinguished: a group of CLBP patients; a group of patients with chronic musculoskeletal pain other than low back pain (e.g., chronic neck pain or shoulder pain), and a group of patients with miscellaneous pain complaints, low back pain and other musculoskeletal pain excluded (e.g., headache, abdominal pain, facial pain).

\section{Method}

\section{Participants and procedure}

Participants in this study were 211 consecutive referrals presenting to a university hospital pain management and research center. All participants had a chronic pain problem (i.e., with a pain duration of at least 6 months). The sample consisted of 75 men and 136 women with a mean age of 48 years $(S D=14.3)$. Three rather homogeneous subgroups of chronic pain patients could be distinguished, taking into account the fact that the subgroups had to be large enough to permit statistical analyses (i.e. regression analyses). Of the total sample, 54 participants (25.6\%) suffered from CLBP, $107(50.7 \%)$ suffered from chronic musculoskeletal pain other than low back, and 50 participants $(23.7 \%)$ had miscellaneous chronic pain problems, back pain and musculoskeletal pain excluded (e.g., headache, abdominal pain, facial pain, thoracic pain). The mean duration of the pain complaints was 6.8 years (SD= 8.4). Of the total sample, $9.5 \%$ were on sick leave, $28.9 \%$ received financial disability compensation, and $61.6 \%$ received no compensation. In addition, $11.1 \%$ of the participants used supportive equipment for ambulation (brace, crutches, corset etc.) and $1.9 \%$ of the participants were dependent on a wheelchair or electric scooter for ambulation. As part of the standard intake procedure participants were asked to complete a number of questionnaires.

\section{Measures}

\section{Physical imparment}

The Medical Examination and Diagnostic Information Coding System (MEDICS) (Rudy, Turk, Brena, Stieg \& Brody, 1990) was used. MEDICS is a method that is designed to quantify the extent of physical findings in chronic pain patients. From this system, a pathology index can be computed, which is a weighted logit score based on 18 common biomedical procedures (e.g., computed tomography scan, muscular function, neurological examination) used in the assessment of the causes of pain in chronic pain patients. For this purpose, a fourth-year medical student, supervised by a neurologist (W.E.I.W. ), examined the medical chart of every patient included in this study before completing MEDICS. The total pathology score, using the medical consensus weights reported by Rudy et al. (Rudy et al., 1990), was used. This score is indicative of the level of playsical impairment.

\section{Pain intensity}

The Dutch language version (DLV) (Lousberg, 1994) of the West Haven Yale Multidimensional Pain Inventory (MPI, formerly the WHYMPI) (Kerns, Turk \& Rudy, 1985) was selected. It is a measure of key aspects of the chronic pain experience, based on the 
cognitive-behavioral perspective. The questionnaire consists of three mijor parts, each containing several subscales. The 13 subseales of the MPI-DLV assess pain intensity, pain-related interference, perceived life control, affective distress, social support, responses of significant others to the patient's pain behaviors, and level of participation in typical daily activities. Psychometric properties of the MPI-DLV are satisfying. The temporal stability $(r=$ .81) and the internal consistency (Cronbach $\alpha=.81$ ) of the pain intensity subsoale is adequate (Lousberg, 1994). Data on construct validity are also adequate. Furthermore, psychonetric properties of the Dutch version are roughly the same as the American version.

\section{Disability}

One of the most widely used instruments for measuring disability, the Roland Disability Questionnaire (Roland \& Morris, 1983), could not be used in this study because it is specifically developed for use with low back pain patients. For practical reasons, the MPI subscales "interference" and. "life control" were used in this study as an indicator for painrelated disability. Flor and Turk (1988) also used the "interference" subscale to measure disability in a study predicting pain and disability from cognitive variables. With respect to content, the items of the "life control" subscale seem to correspond to the World Health Organization definition of disability as well. Therefore it was decided to use both the "interference" and the "life control" subscale as an indicator for disability. The temporal stability of these subscales is adequate $(r=.88$ and .78 , respectively), as is the internal consistency (Cronbach $\alpha=.86$ and .78 , respectively).

\section{Pain catastrophizing}

In this study, we used the Dutch version (an umpublished, 1996 translation by $\mathrm{G}$. Crombez and J.W.S. Vlaeyen) of the Pain Catastrophizing Seale (PCS) (Sullivan et al, 1995). This is a 13item scale in which participants are asked to reflect on past painful experiences and indicate the degree to which they experienced thoughts or feelings during pain on a 5-point scale. Psychometric properties of the Dutch version of the PCS are adequate. It correlates highly ( $r$ $=.73$ ) with the catastrophizing subscale of the Dutch Pain Cognition List (Vlaeyen, Geurts, Kole Snijders \& Schuerman, 1990), has a good temporal stability (Pearson's ${ }^{*}=.92$ ), and a high internal consistency (Cronbach $\alpha=.85$ ) (Geert Crombez et al., 1998b).

\section{Psychological distress}

We used the Dutch version (Arrindell \& Ettema, 1986) of the Symptom Checklist (SCL-90) (Derogatis \& Cleary; 1977; Derogatis, Lipman \& Covi, 1973). This is a 90-item multidimensional state measure of psychopathology. The SCL-90 consists of 8 dimensions: anxiety, agoraphobia, depression, somatic symptoms, distrust and interpersonal sensitivity, anger-hostility, sleeping problems, and general psychopathology/psychoneuroticism, which is expressed in the SCL-90 total score. We used the SCL-90 total score ats a measure of psychological distress. Participants were asked to indicate on a five-point scale to what extend the symptoms as stated in the 90 items applied to them during the past week. Rellability and validity of the Dutch version of the SCL-90 were found to be adequate (Arrindell \& Ettema, 1986).

\section{Statistical analysis}

In addition to physical impairment, pain duration, age, and sex were used in the regression analyses as covariates. A longer duration of pain might go together with more disability and psychological distress. Similarly, besides any pain-related disability, older people might be more disabled because, in general, people develop more health problems with increasing age. The relationship between sex and pain intensity is not unequivocal (see discussion). To rule 
out the possibility that sex influences the relationships of interest, we considered sex as a covariate in the analyses.

Pearson correlations between catastrophizing, control variables (physical impairment pain duration, age, and sex), and dependent variables (pain intensity, interference, life control, and psychological distress), and among independent and control variables were calculated.

We performed two series of stepwise hierarchical multiple regression analyses to examine whether catastrophizing would contribute significantly to the variance in the dependent wariables, after controlling for physical impairment, pain duration, age, and sex, and to examine whether the relationships between catastrophizing and the dependent variables differed for the three subgroups of chronic pain patients. In the first series, we entered the control variables in the equation first, the main effects (catastrophzing and pain group) in the second step, and the interaction terms (pain group $\times$ catastrophizing) in the third step. The pain group $x$ catastrophizing interaction terms were calculated asing dummy-coding. In the second series only the main effect of catastrophizing was tested, after controlling for physical impaiment, pain duration, age, and sex.

Based on the results of the regression analyses, we performed post hoc statistical analyses. Sex differences in self-reported pain were examined with the independent-samples $t$ test. To axamine whether sex moderates the relationship between catastrophizing and pain intensity, we performed a stepwise hierarchical regression analysis with the control variables (age, pain duration, and physical impaiment) entered in the first step, the main effects (catastrophizing and sex) entered in the second step, and the interaction term (sex $\times$ catastrophizing) entered in the third step.

\section{Results}

The mean PCS score for the sample as a whole was 26.47. Correlations between the independent variable (catastrophizing), control variables (physical impairment, pain duration, age, and sex), and dependent variables (pain intensity, interference, life control, and psychological distress) are shown in Table 1.

Table 1.

Conrelations between the independent variable (catastrophizing), control wariables (physical impairment, pain duration, age, and sex), and dependent variabless (pain intensity, interference, life control, and psychological distrosis)

\begin{tabular}{|c|c|c|c|c|}
\hline \multirow[b]{2}{*}{$\begin{array}{l}\text { Independent and } \\
\text { connol variables }\end{array}$} & \multicolumn{4}{|c|}{ Dependent variables } \\
\hline & Pain intensity & Interference & Life control & $\begin{array}{l}\text { Pychological } \\
\text { distress }\end{array}$ \\
\hline Cutawophizing & $.30^{\circ}$ & $.43^{8}$ & $-47^{2}$ & $.54^{2}$ \\
\hline physioal inganment & .14 & .12 & -05 & -.02 \\
\hline Pind duration & -06 &. .05 & $17^{37}$ & -.09 \\
\hline Age & .01 & -.11 & .12 & -.13 \\
\hline Sex & $-2]^{\text {it }}$ & -.12 & .13 & $-16^{4}$ \\
\hline
\end{tabular}

Correlations of catastrophizing with the dependent variables "interference" $(r=43, p<$ $.01)^{2}$ "lite control" $(r=-.47, p<.01)$, and "psychological distress" $(r=.54, p<.01)$ were considerable. Correlation of catastrophizing with pain intensity was smaller but still significant $(r=30, p<.01)$. This indicates that chronic pain patients who catastrophize experience more pain intensity, feel more disabled by their pain problems (i.e., they 
experience more interference of their pain in daily life and experience less control over their lives), and are more psychologically distressed. The correlation between catastropluzing and physical impaiment was not significant $(r=03$, two-tailed, not significant): Otherwise, physical impaiment only correlated significantly with age $(p=.16$, two-tailed, $p<.05)$, although this correlation was only very weak.

The results of the regression analyses demonstrated that there were no significant interaction effects (Table 2), indicating that different subgroups of chronic pain patients do not differ from each other with respect to the relationship between catastrophizing and pain intensity, interference, life control, and psychological distress.

Table 2.

$F$ change walues from the hierarchical regression analyses of the interaction effects (catustrophizing $x$ group membership) with the dependent variables (pain intensity, interferenoe life control, and psychological distress)

\begin{tabular}{lcc}
\hline Dependent variables & $F$ Change & Significance of f change \\
\hline Pain intensity & $1.42(2,172)$ & .24 \\
Interference & $0.03(2,172)$ & .97 \\
Life control & $0.57(2,172)$ & .57 \\
Psychological distress & $0.54(2,171)$ & .58 \\
\hline
\end{tabular}

The results of the second series of regression analyses are summarized in Table 3.

Table 3.

Summary of stepwise herarchical regression analyses of pain intensity, interterence, life control, and psychological distress with physical impaiment, pair duration, age, and sex entered in the first stop and catastrophizing entered in the second step

\begin{tabular}{|c|c|c|c|c|c|c|c|c|c|c|c|c|c|}
\hline \multirow[b]{3}{*}{ Step } & \multirow[b]{3}{*}{$\begin{array}{l}\text { Independent and } \\
\text { control variables }\end{array}$} & \multicolumn{12}{|c|}{ Dependent variablles } \\
\hline & & \multicolumn{3}{|c|}{ Pain intensity } & \multicolumn{3}{|c|}{ Interference } & \multicolumn{3}{|c|}{ Life control } & \multicolumn{3}{|c|}{$\begin{array}{l}\text { Psychological } \\
\text { distress }\end{array}$} \\
\hline & & $\begin{array}{l}\text { Adj } \\
R^{2}\end{array}$ & $R^{2}$ & $\beta$ & Adj & $R^{2}$ & $\beta$ & $\begin{array}{l}\mathrm{Adj} \\
R^{2}\end{array}$ & $R^{2}$ & $\beta$ & $\begin{array}{l}\mathrm{Adj} \\
R^{2}\end{array}$ & $R^{2}$ & $\beta$ \\
\hline \multirow[t]{5}{*}{1} & & 09 & 11 & & .03 & .05 & & .03 & .05 & & .05 & .07 & \\
\hline & Physical mimpaitment & & & $.16^{4 t}$ & & & $15^{f}$ & & & -.08 & & & 00 \\
\hline & Pain duration & & & -.09 & & & .06 & & & $.15^{\prime \prime}$ & & & .08 \\
\hline & Age & & & .05 & & & -.13 & & & .10 & & & -.16 \\
\hline & $\operatorname{Sex}$ & & & $-.29^{\circ}$ & & &. .12 & & & .10 & & & $-166^{11}$ \\
\hline \multirow[t]{6}{*}{2} & & .16 & .18 & & .22 & .24 & & .23 & .25 & & .35 & .37 & \\
\hline & Physical impairment & & & $.15^{t}$ & & & $.14^{8}$ & & & -.07 & & & -.01 \\
\hline & Pain duration & & & -.05 & & & .00 & & & .09 & & & -.01 \\
\hline & Age & & & .00 & & & $-.21^{*}$ & & & $.18^{\prime \prime}$ & & & $-.27^{\circ}$ \\
\hline & Sex & & & $-.27 "$ & & & -.08 & & & .06 & & & -10 \\
\hline & Cattastrophizing. & & & $27^{\circ}$ & & & $.44^{\circ}$ & & & $-47^{4}$ & & & $.57^{*}$ \\
\hline
\end{tabular}

Catastrophizing significantly predicted pain intensity $(\beta=.27, p<.01)$, interference $(\beta=.44$, $p<.01$ ), life control $(\beta=-.47, p<.01)$, and psychological distress $(\beta=.57, p<.01)$, even when controlled for physical impairment, pain duration, age, and sex. Nevertheless, in addition to catastrophizing, physical impaiment significantly added to the prediction of pain intensity $(\beta=.15, p<.05)$ and interference $(\beta=.14, p<.05)$. This means that the patients 
who are more physically impaired, experience more pain intensity and interference from their pain in daily life. In addition to catastrophizing, age significantly added to the prediction of interference $(\beta=-21, p<.01)$, life control $(\beta=.18, p<.01)$, and psychological distress $(\beta=$ $-27, p<.01)$, indicating that older people experience less interference, more life control and are less psychologically distressed. Finally, in addition to catastrophizing, sex significantly and uniquely added to the prediction of pain intensity $(\beta=-.27, p<.01)$, women reporting more pain intensity than men $(t(208)=-3.08, p<.01)$. No interaction effect between catastrophizing and sex was found ( F Change $(1,175)=0.02$, not significant) in predicting pain intensity.

\section{Discussion}

As hypothesized, the current study demonstrated that chronic pain patients who catastrophize experience more pain intensity, feel more disabled by their pain problem, and experience more psychological distress. Overall, catastrophizing is atent predictor of pain intensity, disability, and psychological distress. CLBP patients, patients with chronic musculoskeletal pain other than low back, and patients with miscellaneous chronic pain problems do not differ from each other in this respect. The results of the regression analyses were in strong support of the cognitive-behavioral model of catastrophizing and fear of movement or (re)injury (Vlaeyen et al., 1995b), and demonstrated that physical impairment is not an important confounding variable. Nevertheless, physical impairment makes a modest but still unique contribution to the variance of pain intensity and interference in addition to the contribution made by catastrophizing. Furthermore, in addition to catastrophizing, age uniquely added to the prediction of disability and psychological distress, whereas sex uniquely added to the prediction of pain intensity, with women reporting more pain intensity than men. Apparently, older people feel less disabled by their pain problem and experience less psychological distress. It is plausible that with increasing age, patients come to terms more with their pain problem or simply are less hampered by their disability because of a more restricted range of activities in relation to their age.

The fact that women report more pain intensity than men is in line with the results of a recent study by Keefe et al. (2000), who in addition found the relationship between sex and pain to be mediated by catastrophizing. They explained this by referring to research findings that indicate that pain responses are socialized differently in boys and girls, favoring a more catastrophic style in girls. On the other hand, Edwards, Augustson, and Fillingim (2000) did not find sex differences in self-reported pain. However, they did find, a sex-specific effect of pain-related anxiety on pain intensity, with thighly anxious male patients with chronic pain reporting more pain intensity than low anxious males with chronic pain. This effect was not found among female chronic pain patients. In the current study, howewer, no such moderating effect between sex and catastrophizing was found. Apparently, the relationship between sex and pain is rather complex and yields diverse results in different studies. Clearly, more research on this subject is needed.

The correlation between catastrophizing and physical impairment found in this study is negligibly small $(r=.03$, not significant). This finding is different from the findings of the two studies mentioned in the Introduction, which suggest significant relationships between these two variables (Main \& Waddell, 1991; Reesor \& Craig, 1988). A possible explanation for these divergent results is that the validity of the instrument used to measure objective physical impairment in the above mentioned two studies is questionable. In both studies, objective physical impaiment was determined using the method developed by Waddell and Main (1984), who used regression analysis to select a combination of physical characteristics (such as root compression signs), that gave the best prediction of disability. Each of these 
physical characteristics was given a different loading obtained from the regression coefficients. Combined, an impairment index can be caleulated ranging from $0 \%$ to $40 \%$ (Waddell \& Main, 1984). It can be argued, however, that this method measures functional rather than physical impaiment. Most of the physical charactenistics used in the impairment index (e.g. lumbar flexion in centimeters, root compression signs), are determined on present clinical examination and thus are indicative of the actual and current status of a patient that might, as Reesor and Craig (1988) argued, be more fumetional in nature. Furthermore, by selecting the combination of physical characteristics that have the greatest influence on disability, there might be considerable confounding of the impairment index with disability, which is of course a behavioral (pain) variable.

In the current study the MEDICS system (Rudy et al., 1990) was used to measure physical impairment. It has the advantage that it can be completed from the patients" medical charts. This means that even physical findings dating from the initial phase of the patient's pain problem can be taken into consideration and that the system can be used independently of the patient"s current clinical status.

\section{Conclusions}

In summarizing the major findings of this study, a number of tentative conclusions can be drawn. First, catastrophizing appears to be an important factor in the chronic pain experience as a potent predictor of pain intensity, disability, and psychological distress. There appears to be no differences in this respect between CLBP patients, patients with chronic musculoskeletal pain other than low back, and patients with miscellaneotis chronic pain problems, back pain and musculoskeletal pain excluded. Furthermore, the relationship between catastrophizing and pain intensity, disability, and psychological distress is not confounded by physical impairment, although physical impairment nakes a unique but modest contribution to the prediction of pain intensity and interference. Second, there is no relationship between the level of objective physical impairment and catastrophizing. Third, the results of this study are in support of the cognitive-behavioral model of catastrophizing and fear of movement or (re)injury (Vlaeyen et al., 1995b). Fourth, the MEDICS system as developed by Rudy et al. (1990) appears to be a useful instrunnent for measuring objective physical impairment. The method developed by Waddell and Main (1984), is hypothesized to be a measure of functional impairment.

The findings of this study also have some clinical implications. A general implication is that the role of physical impaiment in the chronic pain experience, modest though it might be, perhaps has become slightly underestimated or neglected in recent years. Given the growing body of evidence conceming the importance of cognitive-behavioral factors in chronic pain, it is probably less obvious to consider a physical cause when a chronic pain patient displays extensive pain behavior and is severely disabled. Nevertheless, in cases of substantial exacerbation of pain and/or interference from pain, it might be advisable to bave patients physically (re)examined. Another implication concerns the classification of patients in relation to customizing treatment. As becomes clear from this study, there appears to be a subgroup of chronic pain patients who, despite different medical diagnoses, are similar: they catastrophize their pain, which is closely related to more pain intensity, more pain-related disability, and more psychological distress in comparison with non-catastrophizing chronic pain patients. Therefore, the PCS might be useful as a screening instrument to select similar subgroups of chronic pain patients for treatment matched to the specific characteristics of these patients. Such an approach is closely in line with Turk (1990), who condemns the socalled "patient and treatment uniformity myths" and advocates a "subgroup customizing" approach. Interestingly, the profile of catastrophizing chronic pain patients (more pain 
intensity, disability, and psychological distress) in this study, closely resembles the profile of the "dysfunctional" subgroup of the multiaxial assessment of pain taxonomy (Turk, 1990).

In conclusion, a number of critical remarks must be made concerning the current study. First, the study is designed in a cross-sectional manner, and positive correlations or regression wights, however significant, are not to be confused with causal relationships. Nevertheless, it is plausible to assume that catastrophizing promotes self-reported pain intensity, disability, and psychological distress, although logically, the opposite might be true as well. In addition, the results of the current study do corrobortute the findings of previously mentioned studies (e.g., Reesor \& Craig, 1988: Vlaeyen at al., 1995b). In this respect the prospective studies by Burton et al. (1995), Klenerman et al. (1995), and Linton et al. (2000), and Linton \& Hallden (1998) support the idea that catastrophizing and pain-related fear are precursors of painrellated disability rather than consequences. Nevertheless, there is a need for experimentally clesigned studies in which the level of catastrophizing is manipulated and the effects of this manipulation on pain variables such as disability, but also on behavioral measures such as approach or avoidance in a behavioral task, are examined.

Second, pain intensity was solely measured with the MPI "pain intensity" subscale, which is a verbal rating scale, whereas it might have been more appropriate to also use a visual analogue scale (Jensen \& Karoly, 1992 ). In addition, the MPI "interference" and "life control" subscales were used as an indicator of pain-related disability instead of using a more direct measure of pain-related disability.

Finally, all of the measures used in this study are self-report measures, which by definition are dependent on a person's subjective judgement and may consequently be subjective to several kinds of bias, such as shared method variance. The use of behavioral measures in combination with self-report measures will strengthen the results of studies on chronic pain. However, a recent study by Jensen, Romano, Turner, Good and Wald (1999) suggests that shared method variance does not entirely explain the relationship between patient-reported pain beliefs and patient-reported measures of functioning (among which is disability).

Regardless of these shortcomings, our study shows that catastrophizing appears to be a crucial cognitive pain variable and probably plays a substantial role in a cognitive-behavioral conceptualization of chronic pain-related disability. 


\section{CHAPTER 5}

Pain catastrophizing and general health status in a large Dutch
community sample

This chapter is a sightly adapted version of the article that is published as:

Severeijns, R. Hout, van den, M.A., Vlaeyen, J.W.S., \& Picavet, H.S.J. (2002). Pain catastrophizing and general health status in a large Dutch community sample. Pain, 99,367 . 376. 


\begin{abstract}
The aim of the current study was to examine the association between pain catastrophizing and general health status in a Dutch adult community sample, including various subgroups of people with musculoskeletal pain in the analyses. For exploratory reasons this study partly replicated previous studies of the factor structure, reliability, and validity of the Pain Catastrophizing Scale (PCS).

Results demonstrated that across different pain subgroups, catastrophizing uniquely contributed variance to the prediction of the various aspects of general health status beyond the variance explained by pain intensity, age, sex, and chronicity. Across subgroups strongest associations were found between catastrophizing and mental health, general health perception, social functioning, and vitality. Furthermore, the association between catastrophizing and the various aspects of general health status was not moderated by the chronicity of the pain.

Results of the confirmatory factor analysis statistically confirmed a three-factor model of the PCS, which was invariant across different subgroups of people with musculoskeletal pain. Inter-factor correlations were high, and the incremental explanatory power of the three-factor model over that of a one-factor model was only marginal. This implies that a one-factor model might be justifiable as well, at least in the general community. Across various pain subgroups the reliability of the PCS total and subscales was adequate. Additional evidence for the concurrent validity of the PCS was found as well.
\end{abstract}




\section{Introduction}

Pain catastrophizing, defined as an extremely and exaggeratedly negative orientation towards pain itself or its possible consequences, is consistently related to pain-related fear and to both physical and emotional health indices such as pain-trelated disability, psychological distress, depression, and pain intensity (Geert Crombez et al , 1998b; Geert Crombez et al., 1999; Flor et al., 1993; Geisser et al, 1994b; Hill, 1993; Hill et al, 1995; Keefe et al., 1989; Main \& Waddell, 1991; Martin et al., 1996; Rudy Severeins, Vlaeyen, wan den Hout \& Weber, 2001 ; Sullivan et all, 1995; Sullivan R'Eon, 1990; Sullivan et al., 1997; Sullivan et al., 1998; Vlaeyen et al., 1995a; Vlaeyen et al., 1995b; Vlaeyen \& Linton, 2000; Wells, 1994). For an extensive overview see Sullivan et al. (2001b). Overall, the results of these studies are consistent with a fear-avoidance model developed by Vlaeyen a al. (1995a; 1995b), in which pain catastrophizing and fear of movement or (re)injury are held to foster the development of chronic pain problems which also have a profound negative influence on general health status. The latter is illustrated by the association of chronic pain with considerable impairments (e.g., restricted walking or mobility), comparatively high disability lewels, and with the enotionall states of anger, demoralization, anxiety, and depression (Latham \& Davis, 1994; Magni et al., 1990; Reesor \& Craig, 1988).

The findings mentioned above suggest a significant relationship between pain catastrophizing and both physical and mental health indices. However, some issues have to be resolved. Although the results of these studies have been based on both clinical and nonclinical samples we are not aware of any studies in which these associations were examined in a large adult community sample. In clinicall samples relatively high base rates of physical and mental pathology would be expected. The non-clinical samples often consist of pain-firee subjects such as students. This may well limit the generalizability of the results to other populations such as non-clinical pain-suffering subjects. Therefore, finding comparable results in a large community sample would yield valuable information on the relationship between pain catastrophizing and general health status and could be helpful to further validate, test, and refine models of risk factors for dysfunction associated with pain problems, such as the fear-avoidance model mentioned previouslly.

Given the importance of catastrophizing in relationt to health status and the development of chronic pain problems, precise and valid measures of this construct are needed. Such measures might serve for instance to identify specific groups at risk of developing chronic pain problems or to identify and select patients for research or for treatrinent matched to specific patient characteristics. Several instruments have been developed, one of which is the Pain Catastrophizing Scale (PCS) (Sullivan et al, 1995), a 13 item self-report measure of pain catastrophizing for use in clinical and non-clinical populations. A number of studies have provided support for a three-factor model for the PCS as well as for it's internal consistency and validity (Osman et al., 2000; Osman, Barrios, Kopper, Hauptimann, Jones \& $O^{\prime}$ Neill, 1997; Sullivan et all., 1995; Sullivan et al., 2000a). The same holds for the Dutch language version of the PCS which was used in the current study (Van Damme, Crombez, Bijttebier, Goubert \& Van Houdenhove, 2002a; Van Damme, Crombez, Vlaeyen, Goubet, Broeck \& Houdenhove, 2000). Although there are no theoretical reasons to expect different results, the Dutch version of the PCS has not been evaluated in a comminity sample yet. Because community samples are frequently included in studies in which the PCS is used for sereening purposes or as a research variable, it is useful to know whether the same psychometric qualities apply as in clinical or non-clinical samples.

In summarizing the above, the aim of the current study was to examine the association between pain catastrophizing and general health status in several subgroups of people with 
musculoskeletal pain in a large Dutch adult community sample. For exploratory reasons the psychometric properties of the Dutch version of the PCS were evaluated as well.

\section{Method}

\section{Participants and procedure}

Data of the Dutch population-based Musculoskeletal Complaints and Consequences Cohort study (DMC 3 -study) (Picavet et al., 2000) were analyzed. The $\mathrm{DMC}_{3}$-study is a nationwide survey of the prevalence and course of musculoskeletal complaints, carried out by the National Institute of Public Health and the Environment in collaboration with Statistics Netherlands. The study consists of two parts. In part one, a questionnaire was sent to a random sample of 8000 persons aged 25 and above, living in the Netherlands, stratified by 10 years age group and sex. The random sample was taken from the population register of 1998. A total of 3664 persons returned the first questionnaire. Due to missing PCS items, the data of 2789 participants were avalable for the current study. Of these, 1247 (44.7\%) were men (mean age, 51.4 years; SD, 14.6 years; range, 53.5 years) and $1542(55.3 \%)$ were women (mean age, 49.2 years; SD, 14.3 years; range, 53.6 years). Of the 2789 participants, 406 (14.6 $\%)$ were unmarried, $2050(73.5 \%)$ were manied, $134(4.8 \%)$ were widowed, and $199(7.1$ \%) were divorced.

In part two of the study, a shortened version of the first questionnaire was sent 6 months later to all respondents of the first part of the study who consented to being reapproached. In the current study, only the data of the first part of the $\mathrm{DMC}_{3}$-study were used.

\section{Measures}

\section{Survey questionnaire}

The survey questionnaire in the first part of the $\mathrm{DMC}_{3}$-study consisted of a 28 pages full color booklet containing general and sociodemographic questions and health-related questions. The health-related questions referred to five different anatomic areas: (1) neck, shoulder or higher part of the back, (2) elbow or wrist/hand, (3) lower part of the back, (4) hip or knee, and (5) ankle or foot. Pages of five different colors corresponded to each anatomic area. Every colored area started with a screening question, asking the participants if they had experienced pain during the past 12 months in the anatomic area concerned. Screen positives were asked to complete all following questions concerning that particular anatomic area. These questions focussed on: anatomic site, whether or not the pain still exists, pain duration and pain intensity, the course of the pain, self-reported causes, specific complaints (e.g radiation to legs for back pain), and some consequences of the pain (e.g., health care utilization, workrelated consequences, use of medicine, limitations in daily life). Furthermore, in answering the research questions the data of three standardized questionnaires were used in the current study: the Dutch version of the PCS, the Dutch version of the RAND-36 Health Survey, RAND-36, and an especially for the $\mathrm{DMC}_{3}$-study adapted version of the Tampa Scale for Kinesiophobia (TSK) (Miller, Kori \& Todd, 1991; Vlaeyen et al., 1995a). These will be discussed into more detail subsequently.

\section{Pain catastrophizing}

The Dutch version (an unpublished, 1996 translation by G. Crombez and J.W.S. Vlaeyen) of the PCS (Sullivan et al., 1995) was used. This is a 13-item scale in which participants are asked to reflect on past painful experiences and indicate the degree to which they experienced thoughts or feelings during pain on a live-point scale, ranging from 0 (not at all) to 4 (always). Three subscales are distinguished: the "helplessness" subscale (items 1, 2, 3, 4, 5, and 12), the "magnification" subscale (items 6,7, and 13), and the "rumination" subscale 
(items $8,9,10$, and 11). Psychometric properties of the PCS appeared adequate in previous research: it correlated highly $(r=.73)$ with the catastrophizing subscale of the Dutch Pain Cognition List (PCL) (Vlaeyen et al, 1990) and had a good temporal stability (Pearson"s $y^{\prime \prime}=$ 92) (Geert Crombez et al., 1999). The internal consistency, measured in a student sample (Geert Crombez et al., 1998b) and in two samples of chronic low back pain patients (see Van Damme et al., 2000) was adequate (Cronbach $\alpha=0.85,0.88$, and 0.91 , respectivelly).

\section{Fear of mowement or (re) injury}

As a measure of fear of movement or (re)injury, Miller et al. (1991) developed the TSK. The Dutch translation (TSK-DV) of the original TSK (Vlaeyen et al, 1995a) was used in this study. It is a four-point Likert-type scale with scoring altematives, ranging from 1 (strongly disagree) to 4 (strongly agree) and consists of 17 items. The total score is calculated after inversion of the individual scores of items $4,8,12$, and 16 . In the adapted version used in this study, the items of the TSK-DV focused on back pain and back injury instead of on pain and injury in general. The questionnaire had to be completed by all participants, whether they had pain or not.

\section{General health status}

The Dutch version of the RAND-36-item Health Survey 1.0, RAND-36 was used. The items of the Dutch version of the RAND-36 are identical to the Dutch translation of the SF-36 (Aaronson, Acquadro, Alonso \& Apolone, 1992). The RAND-36 only differs from the $S F_{-} 36$ scale in the recommended scoring method (van der Zee, Sanderman, Heyink \& de Haes, 1996). It is a 36-item questionnaire including eight subscales: physical functioning (limitations performing daily activities as a result of health problems; ten items), social. functioning (limitations in social activities; two items), role limitations due to physical health problems (problems with work or other daily activities as a result of physical health problems; four items), role limitations due to emotional problems (problems with work or other daily activities as a result of emotional problems; three items), mental health (feelings of depression and nervousness; five items), vitality (feelings of energy and tiredness; four items), bodily pain (the amount of pain and limitations due to bodily pain; two items), and general health (the subjective evaluation of general health status; five items). Perceived health change over the last year is measured with one item. Two dimensions underlie the RAND-36: physical health and mental health. The number of response choices per item ranges from two to six. Standardized scores on each subscale can be computed which range from 0 to $100(100=$ optimal). These scores are used in the statistical analyses. Note that for the subscales role limitations due to physical health problems, nole limitations due to emotional health problems, and bodily pain this means that a higher score indicates less role limitations and less bodily pain. Both the RAND-36 and the SF-36 were found to have adequate psychometric properties (Essink Bot, Krabbe, Bonsel \& Aaronson, 1997; wan der Zee, Sanderman \& Heyink, 1993; van der Zee et al., 1996).

\section{Statistical a malysis}

General health status

Multiple stepwise linear regression analyses were performed with pain catastroplizing as independent variable and the subscales and main dimensions of the RAND-36 entered as dependent variables. Separate analyses were performed for the five subgroups of people with musculoskeletal pain and for the subgroup of people without musculoskeletal pain at baseline. Because PCS total scores deviated from normality and were skewed, with only relatively few people in every subgroup obtaining high scores (see Table 1), we performed a square root transformation. The transformed PCS score was then entered as independent variable in the 
regression analyses: Statistical corrections were made for pain intensity, age, and sex because they are potential confounders. People might experience their general health status to be poorer simply because they experience nore intense pain. Similarly, people generally develop more health problems with increasing age. The relationship between sex and pain is not unequivocal and yields diverse results in different studies (see e.g., Edwards et al., 2000; Severeijns et al, 2001; Sullivan et al., 2000b).

Furthermote, it was hypothesized that chronicity of the pain complaints might moderate the associations between catastrophizing and general health status, these associations being stronger for people with chronic musculoskeletal pain as compared to people with acute or sub acute musculoskeletal pain. This was examined for the five subgroups with musculoskeletal pain following the procedure suggested by Holmbeck (1997) for testing moderated effects. Covariates (pain intensity, age, and sex) and moderator main effect (chronicity) were entered in step 1, the predictor (catastrophizing) was entered in step 2, followed by the interaction between predictor and moderator in step 3. The independent variable and the moderator were centered before testing the significance of the interaction term to eliminate problematic multicollinearity.

\section{Factor struchure}

Confirmatory factor analysis was conducted to evaluate the relative fit of an oblique three-factor model and a one-factor model to the sarmple data. Separate analyses were performed for the sample as a whole, for the five subgroups of people with musculoskeletal pain, and for the subgroup of people without musculoskeletal pain at baseline. Multiple sample analyses were conducted to examine whether the factor structure of the PCS was invaniant across subgroups.

\section{Reliability}

The internal consistency of the PCS total score and subscales was determined with Cronbach $\alpha$-coefficient for the sample as a whole, the five subgroups of people with musculoskeletal pain, and the subgroup of people without musculoskeletal pain at baseline.

\section{Concurrent validity}

Partial correlations between the PCS and the TSK were calculated for the sample as a whole, for the five subgroups of people with musculoskeletal pain, and for the subgroup of people without musculoskeletal pain at baseline. Pain intensity, age, and sex were used as covariates in the analyses. People might be more fearful of movement or injury simple because of more pain intensity. Similarly, older people might be more fearful of movement or injury because they generally are more vulnerable than younger people. With respect to sex the same argument as made earlier applies.

\section{Results}

Table 1 shows the decile and quartile scores, means, and standard deviations of the PCS for the sample as a whole as well as for the different subgroups. The subgroups consisted of people with low back pain, people with pain in the neck, shoulder, or higher part of the back, people with pain in the elbow, wrist, or hand, people with pain in the hip or knee, people with pain in the ankle or foot, and people without baseline musculoskelletal pain. Overall, PCS scores were fairly low, with only relatively few participants in every subgroup obtaining high scores. Lowest PCS scores were obtained in the "no pain" group. No differences were found between people with chronic musculoskeletal pain and people with acute or sub acute musculoskeletal pain with respect to the level of pain catastrophizing (meam PCS score, 11.47 
and 10.58 respectively; standard deviation, 9.95 and 8.98 respectively; $/(1542)=1.26$, not significant). For the group as a whole Pearson correlation between pain catastrophizing and

Table 1 .

Decile scores, quartile scores, means, and standard deviafons (SD) of the Pain Catastrophizing Scale for the sample as a whole (total group) and for difterent pan subgroups

\begin{tabular}{|c|c|c|c|c|c|c|c|}
\hline & $\begin{array}{l}\text { Total } \\
\text { group } \\
\mathrm{N}=2789\end{array}$ & $\begin{array}{l}\text { Low } \\
\text { back } \\
\text { pain } \\
\mathbb{N}=754\end{array}$ & $\begin{array}{c}\text { Neck } \\
\text { shoulded } \\
\text { high back } \\
N=880\end{array}$ & $\begin{array}{l}\text { Elbow/ } \\
\text { Wrist/ } \\
\text { Hand } \\
\mathrm{N}=480\end{array}$ & $\begin{array}{l}\text { Hipl } \\
\text { Knee } \\
N=582\end{array}$ & $\begin{array}{l}\text { Ankle/ } \\
\text { Foot } \\
N=284\end{array}$ & $\begin{array}{c}\text { No pain } \\
\text { group } \\
\mathrm{N}=1164\end{array}$ \\
\hline \multicolumn{8}{|l|}{ Decile } \\
\hline 1 & 0 & 1 & 1 & 1 & 1 & 2 & 0 \\
\hline 2 & 2 & 3 & 3 & 3 & 3 & 4 & 1 \\
\hline 3 & 4 & 5 & 6 & 6 & 5 & 6 & 3 \\
\hline 4 & 6 & 8 & 8 & 8 & 7 & 9 & 4 \\
\hline 5 & 8 & 10 & 10 & $\mathbb{1 1}$ & 9 & 11 & 6 \\
\hline 6 & 10 & 12 & 13 & 14 & 12 & 13 & 11 \\
\hline 7 & 13 & 16 & 16 & 17 & 16 & 17 & 12 \\
\hline 8 & 17 & 20 & 19 & 21 & 20 & 21 & 14 \\
\hline 9 & 23 & 26 & 27 & 29 & 27 & 30 & 19 \\
\hline 10 & 52 & 52 & 52 & 52 & 52 & 48 & 51 \\
\hline \multicolumn{8}{|c|}{ Quartile } \\
\hline 25 & 3 & 4 & 5 & 4 & 4 & 5 & 1.25 \\
\hline 50 & 8 & 10 & 10 & 11 & 9 & 11 & 6 \\
\hline 75 & 15 & 18 & 18 & 18. & 18 & 18 & 12 \\
\hline Mean & 10 & 12.15 & 12.33 & 13.02 & 12.07 & 13.53 & 824 \\
\hline$\overline{S D}$ & 9.25 & 10.41 & 10.30 & 10.76 & 10.41 & 10.98 & 801 \\
\hline
\end{tabular}

pain intensity was moderate $(r=0.30, p=0.01$, one-tailed). For the five subgroups of people with musculoskeletal pain correlations (Spearman's rho) between pain catastrophizing and the course of the pain complaints (ranging from "a single complaint" to "nearly continually severe pain") were low and ranged from 0.04 (not significant) to $0.19(p=0.01$ ).

\section{General health status}

The summary of the results of the linear regression analyses are shown in Table 2. Only six of the 50 tested interaction terms reached significance and these six contributed only very little variance to the prediction of the dependent variables beyond the main effects and covariates (ranging from an additional $0.3 \%$ to an additional $1.0 \%$ ). It appears then that the association between catastrophizing and general health status is not moderated by chronicity or otherwise of the pain complaint. For this reason, the results of step 3 of the regression analyses in which the interaction terms were tested was omitted from the table. The table shows that for the five subgroups of people with musculoskeletal pain, pain intensity, age, sex, and the chronicity of the pain complaint (step 1) together contributed considerable wariance to the prediction of physical functioning and the physical health dimension (ranging from 27 to $35 \%$ ). The tirnique contribution of catastrophizing to the prediction of this subscale and main dimension was rather modest as compared to the other subscales and main dimensions, explaining an additional 5 to $8 \%$ of the variance. Examining the standardized Beta values (not in the table) showed that across different subgroups of people with musculloskeletal pain, older women who had more pain, whose pain complaints were chronic, and who catastrophized about their pain, experienced their physical health to be poorer. 


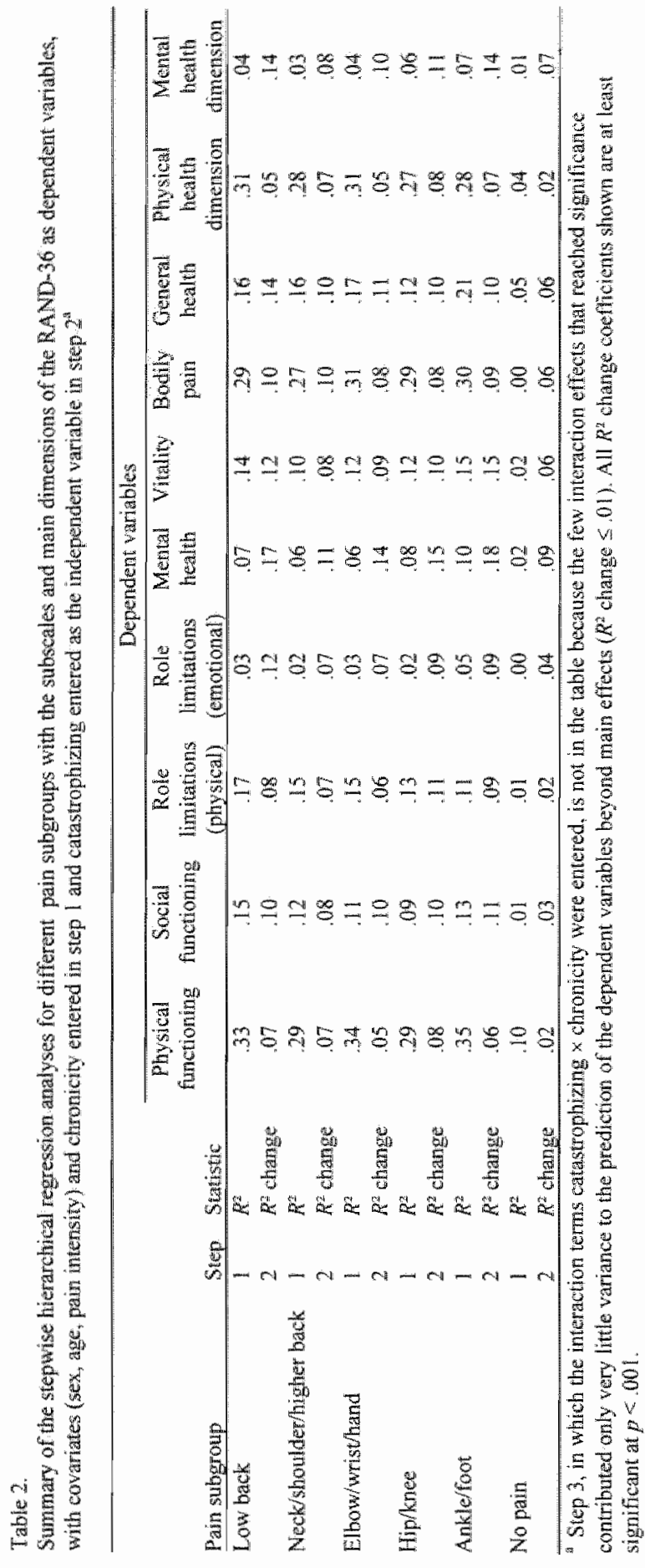


In general catastrophizing particularly contributed unique variance to the prediction of mental health, general health, and the mental health dimension, explaining an additional 8 to $18 \%$ of the variance beyond the variance explained by the combined covariates and moderator variable. Furthermore, for the subscale mental health and the mental healtli dimension, catastrophizing appeared to be a better predictor than the combined covariates and moderator variable.

After examining the standardized Beta values it was found that across different pain subgroups people who catastrophized about their pain, experienced their mental health to be poorer. In general this effect was attenuated somewhat with older age, older people experiencing their mental health to be slightly better. Also, across different pain subgroups older people with chronic pain complaints, who had more pain, and who catastrophized about their pain, perceived their general health to be worse.

Finally, across the five pain subgroups catastrophizing moderately contributed unique variance to the prediction of social functioning and vitality, explaining an additional 8 to $15 \%$ of the variance beyond the variance explained by the combined covariates and moderator variable. Looking at the standardized Beta values it was shown that generally people who had more pain and catastrophized about their pain, experienced more limitations in social activities. Also, in general women who experienced more pain and catastrophized about their pain perceived themselves to be less vital. This effect was attenuated somewhat with older age, older people perceiving themselves to be slightly more vital.

Although still significant, the unique contribution of catastrophizing to the prediction of bodily pain and role limitations due to both physical problems and emotional problems was less prominent across the five pain subgroups.

In the subgroup of people without pain at baseline the associations between catastrophizing and the subscales and main dimensions of the RAND-36 were less prominent compared to the subgroups of people with musculoskeletal pain.

In general, examination of the standerdized Beta values revealled that of the combined covariates and moderator variable, pain intensity in particular contributed unique variance to the prediction of the various aspects of general health status.

\section{Factor structure}

Polychoric correlation matrices were entered in the confirmatory factor analyses. The Weighted Least Squares estimation procedure (WLS) in LISREL 8.30 (Jöreskog \& Sörbom, 1999) for Windows was used to conduct each analysis. Because zero-correlation between factors in the three-factor nodel was highly unlikely, only an oblique model was tested. For this model each PCS item was forced to load on its original factor (i.e., the subscales "helplessness", "magnification", and "rumination"), and the variance of each factor was lixed to 1. The factors were allowed to correlate. In addition, a one-factor model was tested in which all items were forced to load on a single "catastrophizing" factor, and the variance of this factor was fixed to 1. Four goodness-of-fit indices were examined to judge the fit of each model. These indices were: 1) a Non-Nomed Fit Index (NNFI) of .90 or higher, 2) a Comparitive Fit Index (CFI) of .90 or higher, 3) a Goodness of Fit Index (GFI) of .90 or higher, and 4) a Root Mean Square Error of Approximation (RMSEA) (RMSEA <.05: good fit; RMSEA between .05 and under .08 ; reasonable fit; RMSEA between .08 and under . 10 : mediocre fit, and RMSEA > 10: poor fit) (Diamantopoulos \& Siguaw, 2000).

Examining the LISREL output it appeared that correlations among PCS subscales/factors were quite high across all groups, with correlations varying from .87 to 96 . Across all subgroups factor loadings of the PCS items were high, varying from .66 to 98 . 
The goodness-of-fit summary for the models tested is presented in Table 3. As can be seen from the table, both the three-factor model and the one-factor model provided an adequate fit to the data across all subgroups. Overall, the goodness-of-fit indices were slightly better for the three-factor model than for the one-factor model. Because both models are nested, i.e., the same set of parameters are used in both models, and the distribution of $\chi^{2}$ change equals the distribution of $\chi^{2}$, it was possible to statistically compare their adequacy by using the $\chi^{2}$ difference test. The results are shown in Table 4 . The $\chi^{2}$ difference test demonstrated that statistically, the three-factor model for the PCS provided a significantly better fit to the sample data than the one-factor model for all subgroups in the analyses. Because LISREL does not provide percentages total variance explained, the actual difference in explanatory power between a one-factor and a three-factor solution could not be determined. Therefore post-hoc a one-factor and a three-factor solution were examined using SPSS for Windows Release 8.0.2 (SPSS Ine. 1989-1997). The total variance explained was $46.46 \%$ and $49.91 \%$ for the one-factor model and the three-factor model, respectively.

Table 3

Goodness-of-fit und lices for the confimatory factor analyses (Weighted Least Squares estirnation procedure) of whe three-factor model and the one-factor model tot the Paim Catastrophizing Scale

\begin{tabular}{|c|c|c|c|c|c|c|c|c|}
\hline \multirow[b]{2}{*}{ Bain subgroup } & \multicolumn{4}{|c|}{ three-factor mode: } & \multicolumn{4}{|c|}{ one-factor nodel } \\
\hline & NNFI & $\mathrm{CF}$ & GFI & RMBEA & NNFI & CFI & GFI & RMSEA \\
\hline Total group & .96 & 97 & .99 & .056 & .95 & .96 & .99 & .065 \\
\hline Low back pain & 97 & .98 & .99 & .054 & .96 & 97 & .98 & .065 \\
\hline Neok/shouider/higher back & 97 & .98 & .99 & .059 & .96 & .97 & .98 & .068 \\
\hline Elbow/wrist/hand & .98 & .98 & .99 & .066 & .97 & .98 & 98 & .072 \\
\hline Hiphonee & .97 & 98 & .99 & .061 & 96 & .97 & .98 & .072 \\
\hline Analed foot & 97 & .98 & .98 & .063 & .97 & .97 & .98 & .067 \\
\hline Mo nusculoskelctal pain & .96 & .97 & .99 & .061 & .95 & .96 & .98 & .072 \\
\hline
\end{tabular}

Note. The following fit indices are shown: Non-Normed Fit Index (NNFH), Comparative Fid Index (CPI), Coodness of fit Index (CAF) and Root Mean Skuare Fror of Anoroximation (RMSFA .

Table 4

Delta Chi square $\left(\Delta x^{2}\right)$ calculations including level of significance (p) for the three-factor model and the one-factor noded tor the Pan Catastrophizing Scale

\begin{tabular}{|c|c|c|}
\hline Pain subgrous & $\Delta x^{2}(d)$ & $D$ \\
\hline Total group & $227.50(3)$ & $p<.005$ \\
\hline Low back pain & $73.63(3)$ & $p<.005$ \\
\hline Neck/shouldethigher part of back & $76.51(3)$ & $p<.005$ \\
\hline Elbow/wristhand & $36.48(3)$ & $p<.005$ \\
\hline Hip/knee & $62.19(3)$ & $p<005$ \\
\hline Auklefoot & $17.44(3)$ & $p<.005$ \\
\hline No muspuloskelerel pain & $121.31(3)$ & $p<.005$ \\
\hline
\end{tabular}

Note (di) are derrees of freedon

A multi-sample analysis was conducted to examine whether the three-factor model was invariant across subgroups. In line with Van Damme et al. (2002a) the most restrictive model

\footnotetext{
In order to make these andyses comparable to the LISREL analyses the primpal axis actoring method was used with direct oblinin rotation (three-factor nadel only)
} 
was tested, i.e. number of factors, factor loadings, and the correlations between factors were equated across all stbgroups. Because participants could endorse pain in more than one anatomic area, the subgroups reflecting these anatomic areas were not independent, which is a requirement in a multiple sample analysis. Therefore we only included those cases in the analysis whose complaints were limited to that particular subgroup. With this restriction only the low back pain group and the hip/knee group were sufficiently large to be inoluded in the multiple sample analysis. Two other subgroups included in the multiple sample analysis were the group with complaints in more than one anatomic area and the no pain group. The results of the multi-sample analyses demonstrated that the three-factor model provided an adequate fit to the data $(\mathrm{CFI}=99)$ which indicates that the three-factor model is invariant across different subgroups.

One might argue that the results of the confimatory factor analysis obtained in the current study are influenced by the chronicity of pain or by the fact that few participants reported only a single pain location whereas most of the participants reported pain in more than one llocation. For this reason we post hoc conducted additional multi sample analyses. The results demonstrated that the three-factor model for the PCS was invariant across participants with chronic pain and participants with acute or sub acute pain. The same held for participants who reported only a single pain location and participants who reported more than one pain location. This means that both chronicity and amount of pain locations reported did not influence the results of the confirmatory factor analyses.

\section{Reliability}

Cronbach $\alpha$ coefficients for the PCS total and subseale scores were calculated. The internal consistency of the PCS total and subscales was adequate across all groups. For the PCS total, $\alpha$-coefficients ranged from .91 to .93 . For the helplessness subscale they ranged from .83 to .88 , for the magnification subscale they ranged from .71 to .77 , and for the rumination subscale the $\alpha$-coefficients ranged from .85 to .87 . It should be noted that although for the magnification subscale all $\alpha$-coefficients were below 80 , this subscale consists of only 3 items.

\section{Concurrent validity}

Partial correlations (controlling for age, sex, and pain intensity) between the PCS and the TSK across different subgroups were moderate, ranging from .32 to .43 . Although all correlations were significant $(p<.01)$ it should be noted that generally groupsizes were quite large.

\section{Discussion}

Overall, the results regarding general health status imply that pain catastroplizing most prominently has a negative impact on the mental aspects of general health status and on general health. Catastrophizing moderately affects sociall functioning and vitality. More specifically, people with musculoskeletal pain who catastrophize about their pain, experience more feelings of depression and nervousness and in general perceive their mental health to be poorer although this effect is attenuated somewhat as people get older. Older people with chronic pain who experience more pain, and who catastrophize about their pain, evaluate their general health status more negatively. Furthermore, people who have more pain and catastrophize about their pain experience more limitations in sociall activities (e.g. visiting friends or relatives), and women who experience more pain and catastrophize about their pain have less energy and/or are more tired. Pain catastrophizing is less associated with the physical aspects of general health status such as physical functioning and bodily pain or with role limitations due to both physical health problems and emotional problems. In 
summarizing these results it appears that catastrophizing most prominently has a psychological and social impact and to a lesser degree impinges on the level of disability or handicap. These findings are consistent with Vlaeyen et al. (1995b) who found that pain catastrophizing promotes pain-related disability indirectly, via its influence on fear of movement or (re)injury and its associated avoidance behavior and with Severeijns at al. (2001) who found that pain catastrophizing best predicted psychological distress. However, it might also be hypothesized that these results merely reflect the often found relationship between pain catastrophizing and depression (Affleck et al., 1992; Geisser et al., 1994b; Keefe et al., 1989; Sullivan et al 1995; Sullivan \& DEon, 1990). Therefore, future studies on the relationship between catastrophizing and general health status should control for depression. Alternatively, one might argue that the associations between pain catastrophizing and general health status could be explained by the influence of another variable that relates to the medical seriousness of the pain complaints. Although no information about the medical seriousness is available from the current study this alternative explanation seems highly unlikely given the fact that no relationship whatsoever was found between pain catastrophizing and physical impanment in the aforementioned study of Severeins et al. (2001). Furthermore, the associations found in that study between pain catastrophizing and pain intensity, pain related disability, and psychological distress held, irrespective of physical impairment.

The results from the confirmatory factor analyses of the PCS by and large confirm the results of previous studies with the PCS (Osman et al., 2000; Osman et al., 1997; Sullivan et al., 1995 ; Sullivan et al., 2000a; Van Damme et al., 2002a; Van Damme et al., 2000) in which evidence for a three-factor model was found. In the current study we found both the one-factor model and the three-factor model to show adequate fit to the sample data, the later model showing statistically a better fit. However, we also found high correlations among the individual subscales of the PCS which might suggest that these subscales do not really differ from each other and measure virtually the same, namely catastrophizing. In addition, there was only a marginal difference in explanatory power between a one-factor and a three-factor model $(46,46$ versus $49.91 \%$ total variance explained, respectively). This could imply that although the theoretical distinction between helplessness, magnification, and rumination as independent but related dimensions of the catastrophizing construct is supported statistically, a one-factor model of the PCS would be justiliable as well, at least in the general community. One can argue that the fact that a mail out method was used in the current study to gather the data might have obscured somewhat the "true" factor structure of the PCS, i.e. a three-factor model. After all, this method of collecting the research data did not pemit control over the conditions under which the survey questionnaire was completed by the participants. Furthermore, the total number of cases in the analyses represented less than $50 \%$ of the targeted population which means that the sample might not be liree from selection bias. Nevertheless, a still considerable number of people (2789) participated in the current study. Moreover, Osman et al. (2000) also found thigh correlations between subscales of the PCS and high loadings of the subscales on the second-order factor catastrophizing in their community sample. Another point to be made is that PCS scores in the community sample in the current study were generally quite low and even lower than the scores in the community sample study of Osman at al. (Osman et al, 2000). These generally low PCS scores might also have obscured the "true" factor structure of the PCS. On the other hand, low levels of catastrophizing in the general community might be the natural situation. Because previous studies with the PCS have all confirmed a three-factor model it seems valid though to let the statistical argument prevail and to conclude that in the general community the Dutch version of the PCS has a three-factor structure. Nevertheless, because the data also appear to justify a one-factor model, it seems recommendable to be reserved in using the subscales of the PCS 
for screening purposes or as research variables, at least in studies using community samples. A replication of this part of the study in a different community sample would be welcome.

Parallel to Van Damme et all. (2002a) we found this three-factor structure to be invariant across subgroups, indicating that the same processes are measured in different subgroups of pain and in a pain-free sample.

The current study largely confirmed previous studies on the reliability of the PCS. The internal consistency of the PCS total and subscales was adequate for the sample as a whole as well as for the "no pain" group and the different subgroups of people with musculoskeletal pain. Although Cronbach $a$ of the magnification subscale was below .80 across all subgroups, it should be noted that this subscale consists of only three items.

Additional evidence of concurrent validity was found in the current study. There were significant, though moderate correlations (ranging from .32 to .43) between the PCS and a fear-avoidance measure (TSK), irrespective of pain intensity, age, and sex. These correlations were lower than the correlation between catastrophizing and fear of movement or (re)injury found in a study of Crombez et al. (1999) $\left(\mu^{*}=.53\right)$ and also lower than the same correlation found in a study of Vlaeyen et al. (1995b) $(r=.54)$, although in the latter study a different measure of catastrophizing was used. This might be explained by the fact that in the current study mean PCS scores for the different pain subgroups were quite low, ranging from 12.07 to 13.53 while Crombez et al. (1999) found a mean PCS score of 21.7. The rather low PCS scores in the current study might have obscured somewhat the true association between catastrophizing and fear of movement or (re)injury.

This low level of pain catastrophizing in the Dutch population is closely in line with Osman et al. (2000) who found a mean total scale score of 13.87 in the community sample as compared to a mean total scale score of 22.5 for the pain outpatient sample. Apparently, people in the general community are quite different from pain patients seen in clinical or outpatient treatment facilities. A noteworthy finding was that people without musculoskeletal pain obtained the lowest PCS scores (see Table 1). Furthermore, in this group the associations between pain catastrophizing and general health status were less prominent than in the subgroups with musculoskeletal pain (see Table 2). These results might be explained by hypothesizing that those who catastrophize about their pain posses negative pain schema that can be activated by pain and thus lead to cognitive distortions among which pain catastrophizing. The llatter negatively influences the pain experience. It seems then that, just as the depressive schema need a negative life event in order to be activated and to give rise to cognitive distortions (Beck et al., 1979), the presence of pain is a prerequisite for pain catastrophizing to become manifest and exert its adverse influence on the general health status (see e.g. Sullivan et al., $200 \mathrm{lb}$ ). In this respect pain catastrophizing can be considered a latent trait factor. Alternatively, it is conceivable that the inclination to catastrophize about ones pain does not arise until after the actual occurrence of pain. Several factors could potentially influence this inclination to catastrophize: more fundamental personality fraits such as neuroticism/negative affectivity, situational factors such as available medical information or factual medical knowledge of a particular affliction. Future studies should give more understanding of which of these explanations is more likely.

Some critical remarks concerning the generalizability of the results of the confimatory factor analyses have already been made previously. The remark on the shortcomings of a mail out methodology also holds for the association between catastrophizing and general health status. Furthermore, the study design does not permit a judgement on the direction of this association although some prospective studies suggest that catastrophizing is a precursor of pain-related variables such as disability rather than a consequence (Burton et al., 1995; Linton et al., 2000). Finally, all the measures used in the current study were self-report measures which may be subject to several kinds of bias, e.g. shared method variance. 
Notwithstanding these limitations, to our knowledge the current study is the first to defnonstrate in a large community sample that across different subgroups of people with musculoskeletal pain, eatastrophizing about pain is most prominently associated with emotional problems, negative ewaluation of general health status, limitations in social activities, and lack of energy and/or tiredness. In line with findings of previous studies with the PCS, we concluded that in the general community the factor structure of Dutch version of the PCS is best represented by a three-factor model although a one-factor model might be justhtuble as well. Finally, we found additional evidence for internal consistency and concurrent validity of the PCS. 


\section{CHAPTER 6}

Pain catastrophizing is associated with health indices in musculoskeletal pain: A cross-sectional study in the Dutch community

This chapter is a slightly adapted version of the article that is published as:

Severeijns, R., Vlaeyen, J.W.S., Hout, van den, M.A., \& Picavet, H.S.J. (2004). Pain catastrophizing is associated with health indices in musculoskeletal pain: A cross-sectional study in the Dutch community. Health Psychology, 23(1), 49-57. 


\begin{abstract}
Cross-sectional associations were examined between pain catastrophizing and several health indices in 2119 people with musculoskeletal pain from a Dutch community sample. Healih indices included in the current study were specialist consultation, use of medication, and absenteeism or work disiability.

The results demonstrate that for people with a current episode of musculoskeletal pain, pain catastrophizing, pain intensity, and the presence of multiple pain locations were significantly associated with specialist consultation, use of pain medication, and absenteeism or work disability. The authors conclude that the role of pain itself has perhaps been underestimated in recent models of chronic pain-related disability. Some climical implications and suggestions for further research are given.
\end{abstract}




\section{Introduction}

Epidemiological studies have shown that in general chronic pain presents a major public health problem, with estimates of its prevalence ranging from $4 \%$ to $40 \%$ (Andersson, 1994; Blyth, March, Brnabic, Jorm, Williamson \& Cousins, 2001; Bowsher et al., 1991; Brattberg et al., 1989; Crook et al., 1984; Feuerstein et al., 1987; Magni et al., 1990; Sternbach, 1986).

An epidemiological study in the Netherlands (Picavet et al., 1996) concluded that the report of chronic low back pain in the Dutch population increases from 12\% in the 20-29 year age bracket to $21.1 \%$ in the $50-59$ year age bracket. More recently, it was found that over $40 \%$ of the participants in an open Dutch population study reported having any form of chronic musculoskeletal pain (Picavet et al., 2000).

The detrimental consequences of chronic pain in terms of impairment and disability have a large socioeconomic impact due to the high healthcare costs; loss of wages and productivity, and costs of disability benefits and compensation (Bowsher et al., 1991; Feuerstein et al., 1987; Latham \& Davis, 1994; Sternbach, 1986; Turk, 1996b; Turk et al., 1983; van Tulder et al., 1995). This can be illustrated for the Netherlands by the following: In 1991, the total costs of back complaints were estimated at well over 9 billion guilders, which corresponds with $1.7 \%$ of the gross national product (van Tulder et al., 1995; van Tulder et al., 1997). These costs can be divided in direct costs (healtheare, medical, and patient-related costs) and indirect costs (loss of productivity and absenteeism) and were estimated at almost 700 million guilders and 8.6 billion guilders, respectively. Remarkably, about $90 \%$ of the total costs for back complaints are indirect, whereas, normally, direct and indirect costs make up about $70 \%$ and $30 \%$ of total health care costs, respectively (Goossens \& van Tulder, 2000). Cats-Baril and Frymoyer (1991) reported that a relatively small proportion of the back pain patients accounts for most of the costs, namely, the proportion comprising individuals who are permanently disabled; that is, chronic back pain patients represent less than $10 \%$ of all back pain patients but nevertheless account for $85 \%$ of all costs associated with the illness, and they account for $90 \%$ of lost productivity.

From this perspective it is important to gain more insight into the factors that maintain a pain problem and thus contribute to these detrimental consequences. Furthermore, from a clinical point of view, more knowledge about these factors might be instrumental in the early detection of people who are at risk of their pain problem being maintained over time and thus may be at risk of making more use of health care services, of sick leave (absenteeism), or even of work disability.

Over the years, several cognitive-behavioral models have been developed in which fear and avoidance variables were put forward as crucial mechanisms by which acute pain develops into chronic pain (Lethem et al., 1983; Philips, 1987; Waddell et al., 1993). More recently, a cognitively oriented model was presented in which pain catastrophizing and painrelated fear are postulated as central mechanisms in the development of chronic pain problems (Vlaeyen et al., 1995a; Vlaeyen et al., 1995b). In this model, pain catastrophizing, defined as an exaggerated negative orientation toward pain (Sullivan et al., 1995), is conceptualized as a precursor of pain-related fear, more specifically, fear of movement or (re)injury. This in turn is held to promote avoidance behavior and hypervigilance to bodily sensations, followed by disuse, depression, and disability, thus maintaining the pain experiences and reinforcing fear reactions and avoidance behaviors (Asmundson, Norton \& Norton, 1999; Vlaeyen \& Linton, 2000).

There is a growing body of evidence linking pain catastrophizing to pain-related fear and disability, psychological distress, depression, and pain intensity (Geert Crombez et al., 1998b; Geert Crombez et al., 1999; Flor et al., 1993; Geisser et al., 1994b; Hill, 1993; Hill et al., 1995; Keefe et al., 1989; Main \& Waddell, 1991; Martin et al., 1996; Rudy Severeijns et al., 
2001: Sullivan et al., 1995; Sullivan \& D'Eon, 1990; Sullivan et al., 1997; Sullivan et al.; 1998; Wells, 1994). Also, some prospective studies suggest that fear-avoidance beliefs and pain catastrophizing are precursors of pain-related disability rather than consequences (Burton et al., 1995; Klenerman et at., 1995; Limton et al., 2000; Linton \& Hallden, 1998). Overall, the results of these studies are consistent with the fear-avoidance model developed by Vlaeyen et al. 1995a; 1995b), which suggests that pain catastrophizing and fear of movement or (re)injury foster the development of chronic pain problems.

The vast majority of studies on the relationship between pain catastrophizing and pairrelated wariables used selected samples such as patients from an outpatient treatment center or clinical patients. This may have biased the results of these studies because in patient samples relatively high base rates of both physical and mental pathology are to be expected. Therefore, to examine the association between pain catastrophizing and health status on the basis of data from an open adult population sample would greatly support the external validity of earlier findings and of the cognitive model outlined above. The aim of the current study was to examine the relationship between pain catastrophizing and several health indices in an open Dutch population sample. Cross-sectional associations were examined. More specifically, the research question of this study was, Do people who catastrophize about their pain have a higher incidence of specialist consultation for their pain complaints, of using pain medication, and of absenteeism because of their pain complaints or being disabled from work because of their pain?

\section{Method}

\section{Participants and procedure}

Baseline data of the Dutch population-based Musculoskeletal Complaints and Consequences Cohort study (DMC - -study) (Picavet et al, 2000) were analyzed. The DMC $_{3}$-study was a nationwide survey of the prevalence and course of musculoskeletal complaints, carried out by the National Institute of Public Health and the Environment, Bilthoven, the Netherlands, in collaboration with Statistics Netherlands. A questionnaire was sent to a random sample of 8000 individuals living in the Netherlands, ages 25 and over, stratified by 10-year age groups and sex. The random sample was taken from the population register of 1998 . A total of 3664 persons returned the questionnaire. Because of missing Pain Catastrophizing Scale (PCS) items for some respondents, only the data of 2789 participants were available for the current study. Of these, $1247(44.7 \%)$ were men (mean age $=51.4$ years, $S D=14.6$, range $=25.0$ $78.6)$ and $1542(55.3 \%)$ were women (mean age $49.2=$ years, $S D=14.3$, range $=25.0-78.7)$. Of the 2789 participants, $406(14.6 \%)$ were ummarried, $2050(73.5 \%)$ were married, 134 $(4.8 \%)$ were widowed, and $199(7.1 \%)$ were divorced. The highest completed lewel of education was as follows: 427 participants (15.3\%) completed elementary school, 6.12 participants (21.9\%) completed technical and vocational training for 12-16 yeat-olds, 405 participants $(14.5 \%)$ completed school for lower general secondary education, 465 participants (16.7\%) completed technical and vocational training for 16-18 year olds, 228 participants (8.2\%) completed high school, 471 participants (16.9\%) completed technical and vocational training for age 18 and older, and 155 participants $(5.6 \%)$ completed university. Data on the highest completed level of education were missing for 26 participants $(1.0 \%)$.

\section{Measures}

Survey questionnaive

The survey questionnaire consisted of a 28-page full-color booklet containing general and sociodemographic questions and health-related questions. The health-related questions referred to 5 different anatomic regions: (1) neck, shoulder, or higher part of the back, (2) 
ellow or wrist and hand, (3) lower part of the back, (4) hp or knee, and (5) ankle or foot. Pages of 5 different colors corresponded to each anatomic region. Every colored area started with a screening question asking the participants if they had experienced pain during the past 12 months in the anatomic region concemed (e.g." "During the last 12 months, did you experience pain in the neck, shoulders or higher part of the back?"). Panticipans who screen positively on this question were asked to complete all following questions conceming that particular anatomic region. These questions focussed on the anatomic site or sites within every anatomic region in which the participants experienced pain ("Where did you experience pain?"), whether the pain still existed at the time of inquiry ("Do you still have this complaru: currently?"), pain duration ("How long have you had this complaint?"), and current pain intensity ("If you have to express this pain on a scale ranging from 1 to 10 , how bad is it then?"). Current pain intensity was measured with a mumerical rating scale ranging from 1 (some pain) to 10 (worst pain possible).

Pain ratings were awailable for every single specific location within a particular anatomic region. Because they colld endorse pain in more than one specific location, (e.g., screen positives for the first anatomic region -the neck-shoulder-higher part of the back-could report pain in the neck, pain in the shoulder, as well as pain in the higher part of the back). some participants had more than one pain rating for a particular anatomic region. In those cases we used the highest pain rating within that anatomic region as the pain intensity meastre for that participant's anatomic region. For every anatomic region, screen positives also had to indicate whether they currently used pain medication or had used pain medication during the past 12 months, whether they had contacted one or more specialists for their pain complaints during the past 12 months, whether they had been on sick leave because of their pain during the past 12 months, and whether they were receiving disability assistance because of their pain. The questions about specialist consultation, absenteeism, and work disability were dichotomized $(\text { no }=0, \text { yes }=1)^{10}$. Furthermore, the data of a standardized questionnaire that was included in the survey questiontaire was analyzed in the current study: the Dutch version of the PCS. This is discussed in more detail below.

\section{Pain catastrophizing}

The Dutch version (an unpublished, 1996 translation by G. Crombez and J.W.S. Vlaeyen) of the PCS (Sullivan et al, 1995) was used. This is a 13-item scale in which participants are asked to indicate the degree to which they experience thoughts or leelings during pain that are characterized by catastrophizing on a 5 -point scall, ranging from 0 (not at all) to 4 (always). The total score can range from 0 to 52 . Examples of PCS items are (when I'm in pain) "I keep thinking about how much it hurts" or "I wonder whether something serious might happen". The PCS does not have a specific time frame. Psychometric properties of the Dutch version of the PCS are adequate: It has good temporal stability (Pearson's $r=.92$ ) (Crombez et $a 1 ., 1999$ ) and a high internal consistency (Crombez et al, 1998b; Severeijns, van den Hout, Vlaeyen \& Picavet, 2002). Indications for the validity of the Dutch version of the PCS were found as well. (Crombez et al., 1999; Vlaeyen et al., 1990).

10 In answering the question about specialist consultation, participants bad to indicate when they had consulted a specialist for their complaints. If they had consulted a specialisl, they had to select one on more of eight possibilities reflecting the specific specially. Because we wanted to know whether they had consulted a specialist but were not interested in what specific speciatly area they sought consultation, wa dichotomized this variable. Furthemore, the wriables absenteesme and work disability were combined into a composite wariable,

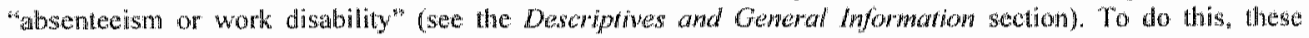
wriables had to be dichotomized as well 


\section{Statisticall anallysis}

Logistic hierarchica] regression analyses were performed to evaluate the associations between the PCS and several health indices. The PCS total scores deviated from normality and were skewed (skewness $=1.29,5 E=.46$ ). To make the solution of the regression analyses more stable and to increase power, we performed a square root transformation. The transformed PCS scores were then entered as independent variable in the regression analyses.

Thre subgroups could be distinguished on the basis of whether participants experienced paint (1) those without pain, neither at the time of inquiry, nor in the year preceding the inguiry (i.e, the group without pain, $n=670,24 \%$ ), (2) those without pain at the time of incuiry but with pain in the year preceding the inquiry (i.e., the group with recent pain, $n=$ $494,17.7 \%$ ), and (3) those with both pain in the year preceding the inquiry and at the time of incuiry (i.e., the group with current pain, $n=1625,58.3 \%$ ). The group with neither current pain nor pain during the previous 12 months was left out of consideration in the subsequent regression analyses. The group with recent pain was considered relevant because the questions about specialist consultation, use of medication, absenteeism, and work disability explicitly referred to the whole period of 12 months before the inquiry (or longer, in case of work disability).

Therefore; we performed separate regression analyses for both the group with current pain and the group with recent pain. The analyses for the group with current pain were adjusted for age, sex, pain intensity, chronicity, and the number of pain regions endorsed. The analyses for the group with recent pain were adjusted for age, sex, and the number of pain regions endorsed. People generally develop more healh problems with increasing age. The relationship between sex and pain is not unequiwocal and yields diverse results in different studies (see e.g., Edwards et al., 2000; Severeijns et al., 2001; Sullivan et al., 2000b). Nevertheless, we wanted to control the possible influence of sex on the results. Similarly, we reasoned that participants with more intense pain or with chronic pain complaints might have a ligher incidence of visiting a specialist, of using medication, and of absenteeism or being work disabled because of their pain complaints. Finally, participants could endorse pain in one or more of five anatomic regions. We reasoned that the number of pain regions endorsed might be positively associated with the health indices and therefore created a new variable: the number of pain regions endorsed, ranging from 0 to 5 . The control variables were entered in the first step of the regression analyses. The transformed PCS total score was entered in step 2 as the independent variable. The health indices served as the dependent variables in the separate regression analyses.

\section{Results}

\section{Descriptives and general information}

The mean PCS total score for the sample as a whole was 10.04. The mean PCS total scores for the subgroups without pain, with recent pain, and with current pain were $7.82,8.81$, and 11.33, respectively. A one-way analysis of variance showed that these means significantly differed from each other, $F(2,2786)=40.6, p<.01$. Post hoc multiple (Tamhane's T2) comparisons revealed that the group with current pain significantly differed from the other two subgroups. Of the group with current pain $(n=1625), 1319$ participants $(81.2 \%)$ suffered from chronic pain (i.e., pain with a duration of more than three months), and 225 participants (13.8\%) suffered from acute or sub acute pain (i.e., pain with a duration of less than 3 months). Both groups did not differ on the level of catastrophizing, $t(1542)=1.26, p=.21$, two-tailed. Data on chronicity were missing for 81 participants $(5 \%)$.

As we mentioned previously, participants could endorse pain in one or more of five different anatomic regions:" pain in the neck, shoulder, or higher part of the back; pain in the 
elbow or wrist and hand; low back pain; pain in the hip or knee; and pain in the ankle or foot. Of those with current pain or with recent pain combined $(n=2119), 821$ participants $(38.7 \%)$ reported pain in one anatomic region, 673 participants $(31.8 \%$ ) reported pain in two anatomic regions, 384 participants (18.1\%) reported pain in three anatomic regions, 165 participants $(7.8 \%)$ reported pain in four anatomic regions, and 76 participants $(3.6 \%)$ reported pain in all five anatomic regions during the past 12 months. This means that there was considerable overlap among the different pain subcategories, with 1298 participants $(61.2 \%)$ reporting pain in more than one anatomic region.

In the current study we considered four health indices: specialist consultation, use of pain medication, absenteeism, and work disability, Of all participants who reported pain during the past 12 months (those with current pain and with recent pain combined), $646(30.5 \%)$ had consulted a specialist for their pain, $608(28.7 \%)$ used medication for their pain, $431(20.3 \%)$ had been on sick leave because of their pain, and $158(7.4 \%)$ were receiving disability assistance because of their pain complaints. As expected, there was some overlap among the different health indices as well. We decided to combine the health indices of absenteeism and work disability into a new composite variable "absenteeism or work disability". "The reason for doing this was that the number of participants who were receiving disability assistance was relatively small (158), composing not more than $7.4 \%$ of all the participants who reported pain during the past 12 months (including those with current and with recent pain). Furthermore, in the Netherlands people are only declared work disabled (receiving disability assistance) after having been on sick leave for at least 1 year.

In Table 1 a correlation matrix is presented of all the nonstrictly categorical variables of interest in the current study. As can be seen in the table, there were significant and positive correlations between catastrophizing and pain intensity, between catastrophizing and the number of pain regions endorsed (in the group with current pain only), and between the number of pain regions endorsed and pain intensity. Also, there were significant and positive associations between catastrophizing, the number of pain regions endorsed, pain intensity and the health indices. The correlations between catastrophizing, the number of pain regions endorsed and the health indices were higher in the group with current pain than in the group with recent pain. Finally, Table 1 shows that the health indices are significantly and moderately interrelated, with phi-coefficients ranging from .32 to .39 in the group with current pain and with phi-coefficients ranging from. 15 to .32 in the group with recent pain.

\section{Catastrophizing and health indices}

The results of the logistic hierarchical regression analyses are shown in Table 2 and 3 , along with odds ratios (ORs) and $95 \%$ confidence intervals. The ORs indicate the relative amount by which the odds for having contacted a specialist, using or having used pain medication, or having been on sick leave or receiving disability assistance increase (OR $>1.00)$ or decrease $(\mathrm{OR}<1.00)$ when the value of pain catastrophizing is increased by $\|$ unit. Because we performed a square root transformation on the PCS total score, a 1-unit increase in the transformed PCS was equivalent to about a 7-point increase in the regular PCS score.

With respect to the group with current pain, the results in Table 2 indicate that participants with current pain who experienced more intense pain, whose pain was chronic, who endorsed more pain regions, and who catastrophized more about their pain had a higher incidence of having contacted a specialist for their complaints (although the OR for age was significant, it was only 1.01). Furthermore, the results indicate that participants with current pain who experienced more intense pain, who endorsed more pain regions, and who catastrophized more about their pain had a higher incidence of using or having used pain medication for their complaints (the OR for age was significant indeed, yet was only 1.01). 


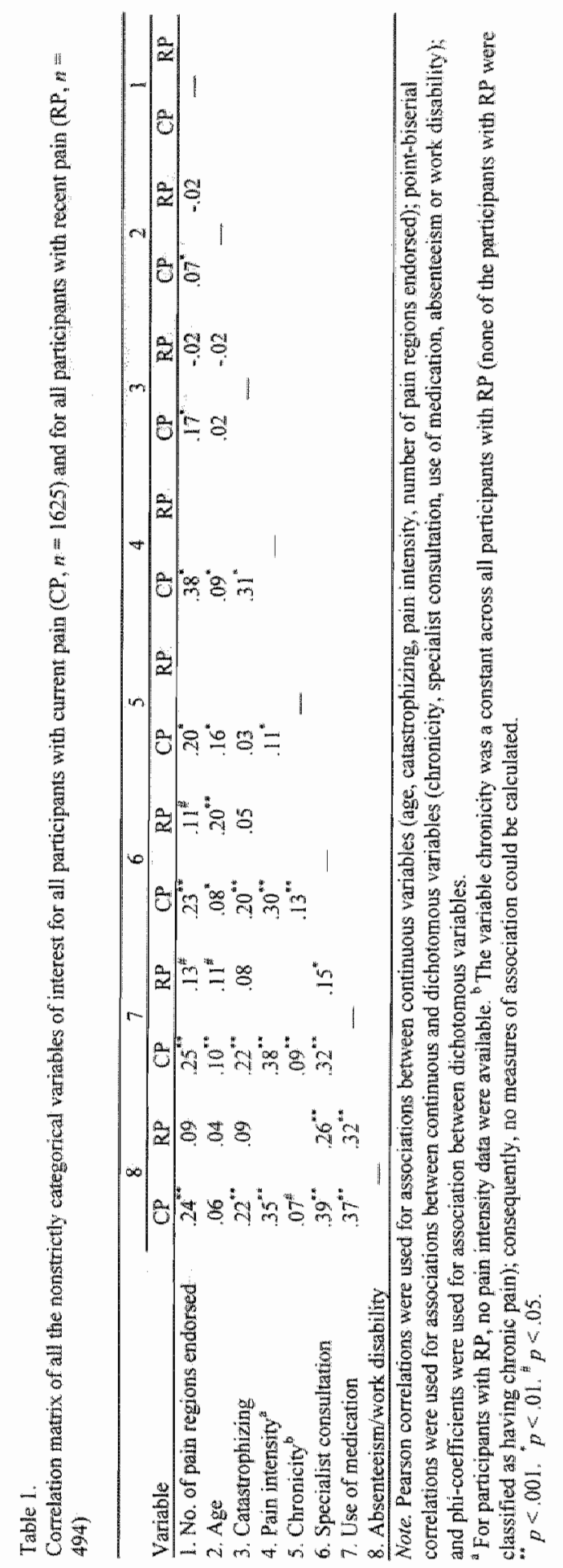


Finally, the results indicate that participants with current pain who experienced nore intense pain, endorsed more pain regions, and catastrophized more about their pain had a highter incidence of absenteeism or work disability.

Table 2.

Results of the logistic hierarchical regression analyses for the group with current pain, with catastrophizing as independent variable and with the health indices as dependent variables, showing odds ratios (OR), 95\% contidence intervals $(\mathrm{Cl})$, and the number of cases in the analyses (

\begin{tabular}{|c|c|c|c|c|c|c|c|}
\hline \multirow{3}{*}{ Step. } & \multirow{3}{*}{ Variables } & \multicolumn{6}{|c|}{ Mesilh indices } \\
\hline & & \multicolumn{2}{|c|}{$\begin{array}{l}\text { Specialisi } \\
\text { consultation } \\
(n=1395)\end{array}$} & \multicolumn{2}{|c|}{$\begin{array}{l}\text { Use of medication } \\
\qquad(n=1411)\end{array}$} & \multicolumn{2}{|c|}{$\begin{array}{c}\text { Absenteeism or } \\
\text { work disability } \\
(n-972)\end{array}$} \\
\hline & & OR & $\mathrm{CI}$ & OR & $\mathrm{Cl}$ & OR & $\mathrm{Cl}$ \\
\hline \multirow[t]{5}{*}{1} & Age & 1.01 & $0.99-1.02$ & $1.01^{\circ}$ & 1.0041 .02 & 1.00 & 0.99 .1 .01 \\
\hline & Sex & 1.19 & $0.94-1.52$ & 1.25 & $0.97-1.61$ & 0.84 & $0.63-1.11$ \\
\hline & Pain intensity & $1.26^{\mathrm{m*}}$ & $1.19-1.33$ & $1.41^{160}$ & $1.33-1.30$ & $1.377^{* * 1}$ & $1.28-1.46$ \\
\hline & Chronicity & $1.86^{4}$ & $1.24-2.77$ & 1.25 & $0.83-1.87$ & 1.05 & $0.69-1.59$ \\
\hline & Number of pain regions endorsed & $1.29^{4 *}$ & $1.16 \sim 1.44$ & $1.32^{* *}$ & $1.17-1.48$ & $1.32^{* *}$ & $1.15-1.51$ \\
\hline \multirow[t]{6}{*}{2} & Age & $1.01^{*}$ & $1.00-1.02$ & $1.01^{\circ}$ & $1.00-1.02$ & 1.00 & $0.99-1.01$ \\
\hline & $\operatorname{sex}$ & 1.18 & $0.93-1.51$ & 1.24 & $0.96-1.6 \|$ & 0.83 & $0.62-1.10$ \\
\hline & Pain untensity & $1.22^{3 *}$ & $1.15-1.29$ & $1.37^{* *}$ & $1.29 \mathrm{~m} 1.46$ & $1.33^{* *}$ & $1.25-1.43$ \\
\hline & Chronicity & $1.90^{*}$ & $1.27-2.84$ & 1.28 & $0.85-1.92$ & 1.07 & $0.70-1.62$ \\
\hline & Number of pain regions endorsed & $1.28^{4 * t}$ & $1.14-1.43$ & $1.29^{\mathrm{kg}}$ & $1.15-1.46$ & $1.28^{* *}$ & $1.11-1.47$ \\
\hline & Catastrophizing & $1.20^{\circ *}$ & $1.11-1.30$ & $1.20^{* *}$ & $1,10-1,3 \mathbb{1}$ & $1.19^{* *}$ & $1.08-1.31$ \\
\hline
\end{tabular}

Nore. All analyses were adjusted for age, sex, pain intensity, chronicity, and number of pain regions endorsed. The $n$ "s across the three thalth indices are not the same. This is mainly due to the fact that not all the hesalth indices were applicable to all participants which resulted in user-missing values. Also, there might be systemmissing values for one or more of the other variables in the model. Cases with one or more missing vallues on any of the variables in the model are automatically excluded from the analyses.

$" p<0.001 ; " p<0.01 ; " p<0.05$; all other are not significant.

For participants with recent pain Table 3 shows that no significant associations between catastrophizing about pain and any of the health indices were found, although the ORs for use of medication and absenteeism or work disability approached the level of significance ( $p s$ .06 and .07 , respectively). It should be noted that the sample size of the group with recent pain was quite smaller than the sample size of the group with current pain, which reduced power and consequently affected significance level. With respect to the covarites, significant associations between the number of pain regions endorsed and specialist consultation and the use of medication were found. This means that participants with recent pain who endorsed more pain regions had a higher incidence of having contacted a specialist and of using of having used pain medication for their complaints. Also, a significant association between sex and the use of medication was found, indicating that women with recent pain had a higher incidence of using or having used pain medication Indeed, significant associations were found between age and specialist consultation and use of medication, yet the ORs, 1.04 and 1.03 , respectively, were barely higher than 1.00 ).

Because the first series of regression analyses demonstrated unique effect of catastrophizing on the health indices for the growp with current pain as a whole, even when adjusting for age, sex, pain intensity, chronicity, and the number of pain regions endorsed, we decided to examine the five pain subcategories separately. 
Table 3

Results of the logistic hiterarchical regression andyses for the growp with irecent pain, with catastrophizing ats the independent variable and with the health indices as dependent variables

\begin{tabular}{|c|c|c|c|c|c|c|c|}
\hline \multirow[b]{2}{*}{ Step } & \multirow[b]{2}{*}{ Variable } & \multicolumn{6}{|c|}{ Health indices } \\
\hline & & \multicolumn{2}{|c|}{$\begin{array}{c}\text { Specialist } \\
\text { consultation } \\
(n=454)\end{array}$} & \multicolumn{2}{|c|}{$\begin{array}{l}\text { Use of medication } \\
\qquad(n=480)\end{array}$} & \multicolumn{2}{|c|}{$\begin{array}{c}\text { Absenteeism or } \\
\text { work disability } \\
(n=316)\end{array}$} \\
\hline \multirow{4}{*}{1} & & OR & $95 \% \mathrm{Cl}$ & $\mathrm{OR}$ & $95 \% \mathrm{Cl}$ & $\mathrm{OR}$ & $95 \% \mathrm{Cl}$ \\
\hline & Age & $1.04^{4 * 4}$ & $1.02 \sim 0.06$ & $1.03^{\circ}$ & $1.01-1.05$ & 1.01 & $0.99-1.91$ \\
\hline & $\operatorname{sex}$ & 0.68 & $0.40-1.17$ & $2,01^{*}$ & $1.18-3.41$ & 1.00 & $0.60-1.66$ \\
\hline & Number of pain regions endorsed & $1.63^{i k}$ & $1.14-2.34$ & $1.55^{\prime \prime}$ & $1.11-2.18$ & 1.35 & $0.95-1.91$ \\
\hline \multirow[t]{4}{*}{2} & Age & $1.04^{4 * *}$ & $1.02-1.06$ & $1.03^{*}$ & $1.01 \cdot 1.05$ & 1.01 & $0.99 * 1.03$ \\
\hline & Sex & 0.65 & $0.38-1.12$ & $1.89^{6}$ & $1.12-3.27$ & 0.96 & $0.58-1.51$ \\
\hline & Number of pain regions endorsed & $\mathbb{1} .66^{\prime \prime \prime}$ & $1.15-2.38$ & $1.59^{\circ}$ & $1.1 \div-2.20$ & 11.37 & $0.93-1.88$ \\
\hline & Catastrophizing & 1.15 & $0.95-1.39$ & 1.19 & $0.99-1.43$ & 1.19 & $0.97 \times 1.42$ \\
\hline
\end{tabular}

None. All analyses were adjusted for age, sex, and number of pain regions endorsed. The number of cases ( $n$ ) across the three thealth indices are not the same. This is mainly due to the fact that not all the bealth indices were applicable to all participants whith resulted in user-mistring walwes. Also, there might be system-missing values for one or more of the other warables in the model. Cases with one or more missing values on any of the variables in the modes are atomatically excluded from the andyses.

"*t $p<.001 ; " p<.01 \% " p<.05$; all other are not significart.

Separate logistic regression analyses were performed for each of the five pain subcategories with current pain. The results of these analyses are shown in Table 4.

Table 4 .

Summary of the logistic thierarchical regnession andyses with catastrophizing as independent variable and the health indices as dependent wariables

\begin{tabular}{|c|c|c|c|c|c|c|c|c|c|}
\hline \multirow{3}{*}{ Pain subcalegory } & \multicolumn{9}{|c|}{ Health indices } \\
\hline & \multicolumn{3}{|c|}{ Specialist consultation } & \multicolumn{3}{|c|}{ Use of medication } & \multicolumn{3}{|c|}{$\begin{array}{l}\text { Absenteensm or work } \\
\text { disability }\end{array}$} \\
\hline & OR & $\mathrm{Cl}$ & $n$ & $\mathrm{OR}$ & $\mathrm{CI}$ & $n$ & OR & $\mathrm{Cl}$ & $n$ \\
\hline Neck/shoulder/higher parl of back & $1.14^{13}$ & $1.02-1.28$ & 700 & $1.20^{\circ}$ & $1.07-1.35$ & 731 & 1.15 & $0.97-1.36$ & 399 \\
\hline Elloww/wrisu/hand & 1.15 & $0.98 \cdot 1.35$ & 373 & 1.16 & $0.97 \times 1.39$ & 393 & $1.40^{\circ}$ & $1.09-1.78$ & 202 \\
\hline low back & & $1.11-1.43$ & 679 & $1.26^{*+4}$ & $1.11-1.43$ & 681 & & $1.14-1.55$ & 428 \\
\hline & $1.26^{*}$ & & & & & & $1.33^{* *}$ & & \\
\hline Hip/kner & $4.31^{\circ}$ & $1.12-1.53$ & 431 & $1.53^{*}$ & $1.28-1.81$ & 459 & $1.37^{2}$ & $1.06-1.76$ & 225 \\
\hline Anklatool & $\|\|$, & $0.90 \times 1.36$ & 212 & 1.26 & $0.99+1.59$ & 219 & 1.11 & $0.79-1.56$ & 99 \\
\hline
\end{tabular}

Nore, All andyses were adjusted lor sex, age, the number of pain negions endorsed, pain intemsity, and shronicity and ware limaled to participants who currently reported pain. $O R=$ odds talio, $C l=$ confidence interval.

As can be seen in Table 4, significant effects were found across the five different pain subcategories with current pain. The results shown in the table might imply that there ara some differences between the five pain subcategories with respect to the relationship between catastrophizing and the health indices. However, because the different pain subcategories do not comprise independent samples, no direct statistical comparisons can be made. Therefore, we performed a post hoc logistic regression analysis, in which we examined whether the 
relationship between catastrophizing and the health indices was moderated by the specific type of pain problem (i.e., each of the five different pain regions). In Step 1 of the analysis the covariates (age, sex, pain intensity, and chronicity), catastrophizing and the newly created variable pain location were entered. Before performing the analyses we specified the variable pain location as categorical so that it would be automatically dummy coded by the SPSS computer program. In the second step, the interaction term catastrophing $x$ pain location was added. The analysis was limited to participants with current pain who had endoised onlly a single pain region. However, the results revealed that none of the interaction terms reached signiffcance, which indicates that for participants with current pain in only one anatonic region, the association between catastrophizing and the health indices was not moderated by the specific type of pain.

Furthermore, the data demonstrate (independent from the table) that, across the different pain subcategories particularly, participants who experienced more pain had a higher incidence of specialist consultation, use of medication, and absenteeism or work disability ( 14 out of 15 ORs were significant, varying from 1.21 to 1.61 ).

\section{Discussion}

Our primary objective was to determine whether there was a relationship between catastrophizing and several health indices in a Dutch adult community sample. This relationship was confimed for people who were currently experiencing muscaloskeletal pain but not for people with a recent history of musculoskeletal pain who were not currently expertencing pain. Also, people with current pain reported significantly higher PCS scores than people with a recent history of pain or people with no pain at all. Apparentlly, the presence of pain is a prerequisite for pain catastrophizing to become manifest. In this respect, catastrophizing about pain might be considered a latent trait factor that can be activated by the presence of pain. Furthermore, pain intensity might function as a moderator variable. However, post hoc analyses in which this pain intensity moderator effect was tested (i.e., the PCS $\times$ pain intensity interaction) did not yield a significant effect. Besides the predictive value of pain catastrophizing, pain intensity and the presence of multiple pain locations independently predicted health outcomes. This could imply that the role of pain and pain intensity has been rather underestimated in recent models of chronic pain-related disability such as the fear-avoidance model (Vlaeyen et al., 1995a; Vlaeyen et al., 1995b). Clearly, further research is necessary.

Looking more closely at the results in Table 4 suggests that the associations between pain catastrophizing and the health indices examined could perhaps be moderated by the specific type of pain. Unfortunately, because of the overlap between the five pain subgroups this hypothesis could not be statistically tested directly. When the analyses were limited to participants with "pure" pain, that is, with current pain in only one anatomic region, however, no statistical evidence for a pain location moderator effect was found. It should be noted though that the current study also demonstrates that in the general community, people with pain in only a single anatomic region are in the minority and that the presence of multiple pain problems is probably the more natural situation. A more definite answer could come from a study in which this moderation effect is examined in a clinical sample of people with one or more pain complaints who are classified into different pain subgroups on the basis of their primary pain complaint (e.g., determined by reason for referral).

The PCS score distribution demonstrated that overall the level of pain catastrophizing in the Dutch population was quite low, with a mean PCS total score for the sample as a whole of 10.04. This is closely in line with a study by Osman et al. (2000) who found a mean PCS total score of 13.87 in an adult community sample as compared with a mean total scale score of 
22.25 for a pain outpatient sample. This implies that people in the general community are probably quite different from clinical samples, at least as far as the level of pain catastrophizing is concerned. It is likely that the low PCS scores in this community sample were of influence on the associations between pain catastrophizing and the health indices and that in a clinical sample these associations would even be more pronounced Replication of this study with clinical samples should yield the necessary information. Nevertheless, the fact that despite these generally low PCS scores significant associations between catastrophizing and the health indices were found makes the results even more meaningful.

Taken together, the results of the logistic regression analyses may have some clinical implications for those currently experiencing a pain episode. First, for people with a current episode of musculoskeletal pain catastrophizing about pain was associated with a higher incidence of visting a specialist, of using medication, and of absenteeism or work disability even when adjusting for pain intensity, chronicity, and the number of pain regions endorsed. This may indicate that the PCS can be a useful instrument in identifying those at risk. However, replication of these associations in a prospective design should provide more definite support of the usefulness of the PCS in screening people with current musculoskeletal pain.

Second, reducing the level of pain catastrophizing in those at risk (i.e., those who currently are experiencing a pain episode and catastrophize about their pain) might lead to less medical consumption, less absenteeism, and perhaps even reversibility of work disability which in turn might lead to a considerable reduction in health care costs. Reducing the level of pain catastrophizing could be achieved by presenting at-risk and affected individuals with a cognitive-behavioral therapy program in which, for example, the rationale of the fearavoidance model of Vlaeyen et al. (1995b) is explained in detail, urrational beliefs about pain are challenged, and behavior experiments are carried out. In this respect, preliminary evidence exists that such a specific and tailored cognitive-behavioral intervention is highly effective (Vlaeyen, de Jong, Geilen, Heuts \& van Breukelen, 2001.). Future studies should yield more information on these matters.

Finally, some critical remarks have to be made concerning the generalizability of the results of the current study. First, in the current literature on catastrophizing there has been discussion on whether catastrophizing is to be considered a separate construct or is confounded with depression (Sullivan \& D'Eon, 1990). If the latter is the case, the results of the current study would perhaps be explained better in terms of the participants' level of depression. However, no measure of depression was used in the current study, which can be considered a conceptual and methodological limitation. Without repeating the whole debate, we will limit ourselves to the comment that although catastrophizing and depression are related, there are convincing arguments coming from several studies to consider catastrophizing as a separate construct (Geisser et al., 1994a; Geisser et al, 1994b; Haaga,

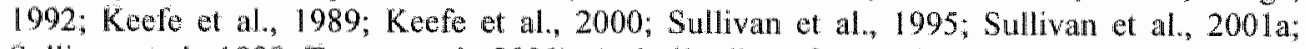
Sulliwan et al., 1998; Turner et al., 2000). A similar line of reasoning concerns the disposition of neuroticism or negative affect, of which a number of authors feel that catastrophizing is a part (for an overview of this discussion, see Tumer \& Aaron, 2001). Nevertheless, as with depression, several studies have shown that although related with negative affect, catastrophizing has incremental value as a predictor of several pain-related outcome variables over negative affect (Affleck et al., 1992; Drossman et al., 2000; Martin et al., 1996; Sullivan et al., 1995). Thus, although no measure of negative affect was used in the current study, it seems unlikely that it would account for all the results found.

Second, although the results of the regression analyses suggest that pain catastrophizing contributes to more health care consumption and absenteeism or work disability, no causal 
atributions can be made because the study design is cross-sectional. Finding similar results in a prospective design would certainly enhance the vallidity of these conclusions.

Third, the vast majority of participants who currently reported pain suffered from chronic pain $(81.2 \%)$. Although we argued at the beginning of this article that it is likely that catastrophizing precedes and contributes to chronic pain, one might also argue that catastrophizing stems from chronic pain. The cross-sectional nature of this study does not allow a definitive judgement on this matter. Nevertheless, the results of the curent study might provide a clue. If catastrophizing stems from chronic pain, one would expect higher levels of catastrophizing for participants with chronic pain as compared with those with acute or sub acute pain. However, in the current study no differences in the level of catastrophizing were found between these two groups.

Fourth, all the measures used in this study are self-report measures or single retrospective questions, which are subject to several kinds of bias. Future studies should also include other and, if possible, more objective measures such as ratings by clinicians or behavioral measures.

Fifth, a mail-out methodology was used in the current study to gather the data, which does not permit control over the way participants complete the questionnaire. This might have infuenced the results. Furthermore, although the sample was quite large (2789), the actual response rate was $46 \%$ (the questionnaire was sent to 8000 people) and an additional $10 \%$ of the data could not be used due to missing PCS items. This means that selection bias cannot be excluded.

Notwithstanding these limitations, to our knowledge, the current study is the first to demonstrate in a large community sample, that people with a current episode of musculoskeletal pain who catastrophize about pain, have a higher incidence of specialist consultation, use of pain medication, and absenteeism or work disability. The same holds for people with current pain who experience more intense pain and for people with current pain who have multiple pain complaints. 


\section{CHAPTER 7}

Pain catastrophizing and consequences of musculoskeletal pain: A prospective study in the Dutch community

This chapter is published as:

Severeijns, R., Vlaeyen, J.W.S., van den Hout, M.A., \& Picavet, H.S.J. (2005). Pain catastrophizing and consequences of musculoskeletal pain: A prospective study in the Dutch community. Jownal of Pain, 6(2), 125-132. 


\begin{abstract}
Elaborating on previous prospective and cross-sectional research, the primary aim of this study was to examinc in the general community whether pain catastrophizing predicts the development of chronic pain complaints and other consequences of pain. The following health indices were examined as consequences of pain: specialist consultation, use of pain medication, and absenteeism. It was also exammed whether these relationships were moderated by the number of pain problems and by pain intensity.

The results demonstrated a generally low level of catastrophizing and a small but significant effect of catastrophizing on the development of chronic pain complaints. With respect to the health indices, no significant effects of catastrophizing were found, nor were the relationships between catastrophizing and chronicity and the health indices moderated by the number of pain problems or by pain intensity.

Because in the general community the level of catastrophizing is low, its role in the development of future pain problems is probably limited in this type of setting. More practically, the Pain Catastrophizing Scale (PCS), used to measure pain catastrophizing, is probably of limited use as a screening instrument in the general community. The disappointing results may indicate that, dependent on the specifie setting (e.g., clinical, oupatient, or community) the role of pain catastrophizing is either more or less prominent.
\end{abstract}




\section{Introduction}

At present it is generally accepted that, especially in chronic pain, the association befween physical impairment and pain intensity and pain-related disability is ouly moderate and that psychosocial factors play a crucial role in the etiology and maintenance of pain-related problems (Feuerstein et al., 1987; Rudy Severeijns at al, 2001; Turk, 1996a; Tuk \& Rudy. 1992; Vlaeyen et al, 1995b; Waddel, 1987; Waddell \& Man, 1984; Waddell, Mam, Morrs: Di Paola \& Gray, 1984; Waddell et al., 1993; Waddell et al., 1989). This is expressed in a recently developed fear-avoidance model of chronie pain (Vlaeyen et al, 1995: Vlacyen al., 1995b). In this model pain catastrophizing plays a role in the transition from an aoute 10 a chronic pain problem. People who catastrophize about pain have extremely and exaggeratedy negative beliefs about pain, thinking the worst about pain and appraising pain as very threatening. The fear-avoidance model states that if pain, possibly of somatic origin, is catastrophized about, pain-related fear develops. This in tum leads to avoidance behaviors and hypervigilance to bodily sensations, and is followed by disuse, depression, and disability, thus preserving the pain experience (Vlaeyen \& Iinton, 2000).

In recent years evidence has been cumulating that there are associations between pain catastrophizing and various pain outcomes across numerous chical and non-chical populations and that it also is associated with outcome variables in expermertally induced paim (for an extensive overview, see Sullivan et al., 2001b). The bulk of these studies ate cross-sectionally designed although there are some prospective studies, using clinical samples, that found a significant relationship between pain catastrophizing and pain outcome variables such as pain intensity, disability, and depression (e.g., Burton et al, 1995; Drossman et al., 2000; Jensen, Ehde, Hoffman, Patterson, Czerniecki \& Robinson, 2002; Jensen at al. 2001; Keefe et al., 1989; Linton et al., 2000; Samwell et al., 2000).

In this respect two issues are important. First, little is known about the possible role of catastrophizing in the development of chronic complaints and of the prospective relationship between pain catastrophizing and several pain outcomes in the general population. This is an important void because in clinical samples relative high base rates of both physical and mental pathology can be expected and the frequent use of pain-free participants in nonclinical samples limits the generalizability of the results to other populations such as nonclinicall pain-suffering subjects. In this respect two studies are relevant. Both studies used the same dataset. The first study, a prospective population-based study carried out in the Metherlands (Picavet et al., 2002) showed that pain catastrophizing significantly predicted the development of chronic low back pain complaints over a 6 -months period. However, the study was limited to people with low back pain. particinants who already had chronic low back pain at baseline were included in the analysis as well (although the analysis was corrected for baseline chronicity), and tertille scores of the Pain Catastrophizing Scale (PCS) for measuring pain catastrophizing were used insted of continuous scores. The second study. a cross-sectional population-based study, demonstrated that there were small but significant cross-sectional associations between pain catastrophizing and specialist consultation, use of pain medication, and absenteeism or work disability (Severeijns, Vlaeyen, van den Hout \& Picavet, 2004). In the current study with the same data set, we will further elaborate on these two studies (Picavet et al., 2002; Severeins al al, 2004) and examine the prospective relationships between pain catastrophizing and the development of chronic complaints, specialist consultation, use of pain medication, and absenteeism in a sample with musculoskeletal pain in general. A more stringent method than that in the Picavet et al. study (2002) will be used.

Second, from epidemiological studies there are indications that the presence of multiple pains is related to several pain outcomes such as disability, depression and the development of 
more pain symptoms (Crof, 1996. Dworkin, Von Korff \& LeResche, 1990; Urwin; Symmons, Allison, Brammah, Busby, Roxby, Simmons \& Williams, 1998). Also, a relationship between pain intensity and catastrophizing about pain has been consistently reported in several studies (see Sullivan et al, $200 \mathrm{Ib}$ ). Because catastrophizing is about threat; these results might imply that having more intense pain or having multiple pain problems enhances the threal value of painful stimuli and thus affects the relationship between catastrophizing and outcome variables such as the development of chronic complaints. Therefore, it will also be examined whether the relationships between pain catastrophizing and the development of chronic pain complaints, specialist consultation, absenteeism, and use of medication only hold under specific pain-related conditions, namely the presence of multiple pain complaints, and the presence of more intense pain.

Summarizing the foregoing, in the current study two hypotheses are tested: 1) the hypothesiss that pain catastrophizing predicts the development of chronic pain complaints, specialist consultation, the use of pain medication, and absenteeism and 2) the hypothesis that the relationship between pain catastrophizing and these variables is moderated by the number of pain regions endorsed and by pain intensity. The study will include people with musculoskeletal pain in general from a community sample.

Confirming either hypothesis could help to further specify and identify the people at risk for development of chronic musculoskeletal pain, for consulting specialists, for using pain medication, and for work absenteeism. This in turn might contribute to the prevention of chronic pain problems and to a reduction in health care costs by development of treatment programs for those at risk, specifically aimed at reducing pain catastrophizing. From a more theoretical view the results of this study may shed some light on the specific conditions under which pain catastrophizing is active in the fear-avoldance model as outlined above.

\section{Method}

\section{Participants and procedure}

Data of the Dutch population-based Musculoskeletal Complaints and Consequences Cohort study (DMC 3 -study) (Picavet et al., 2000; Picavet and Schouten, 2003) were analyzed. The $\mathrm{DMC}_{3}$-study was a nation-wide survey of the prevalence and course of musculoskeletal complaints, camried out by the National Institute of Public Health and the Environment in collaboration with Statistics Netherlands. The study was approved by the medical-ethical test committee (TNO METC-judgment 98/41). The study consisted of two parts. In part 1, a questionnaire was sent to a random sample of 8000 individuals aged 25 and over, living in the Netherlands, stratified by 10 -year age group and sex. The random sample was taken from the population register of 1998 . In a covering letter the participants were informed that the data would be used for research purposes. By completing and rentring the questionnaire participants gave their consent. A total of 3664 persons returned the first questionnaire. Because of missing PCS items, data of 2789 participants were available for analysis.

In the second part of the study (six months after the baseline measurement), an abridged version of the baseline questionnaire was sent to all respondents of the first part of the study who had consented to being approached for the follow-up (this was a separate question in the baseline questionnaire). Follow-up data of 1888 participants were available for the current study. Of these 1888 participants $838(44.4 \%$ ) were men (mean age $=51.8$ years, $\mathrm{SD}=14.3$, range from 25 to 78.6 years) and $1050(55.6 \%$ ) were women (mean age $=48.8$ years, $\mathrm{SD}=$ 14 , wange from 25 to 78.6 years). Of the 1888 participants 261 (13.8\%) were unmarried, 1403 $(74.3 \%)$ were married, $89(4.7 \%)$ were widowed, and $135(7.2 \%)$ were divorced. 


\section{Measures}

\section{Survey questionnaine}

The survey questionnaire consisted of a 28 pages full color booklet containing general and health-related questions. The health-related questions referred to five different anatomic aress, each referring in tum to two or three of ten specific anatomic sites (1. neck, shonlder or higher part of the back, 2. elbow or wristhand, 3. lower part of the back, 4. hip or knee, 5. ankle or foot). Pages of five different colors corresponded to each anatomic area. Every colored area started with a screening question, asking the participants if they had had pain during the past 12 months in the anatomic region concerned (e.g. "during the past 12 months, did you experience pain in the neck, shoulders or higher part of the back?"). Screenpositives were asked to complete all following questions concerning that particular anatomic region. These questions focused on: the particular anatomic pain site or pain sites within every anatomic region ("where did you experience pain?"), whether or not the pain still exists at the time of inquiry ("do you still have this complaint currently?"), pain duration ("how long do you have this complaint already?") and current pain intensity ("if you have to express this pain on scale ranging from 1 to 10 , how bad is it then?"). The latter was measured with a numerical rating scale ranging from 1 (some pain) to 10 (worst pain possible). Pain ratings were available for every single specific location within a particular anatomic region, as mentioned above. Because participants could endorse pain in more than one specific location, (e.g., screen positives for the first anatomic region, i.e, the neck/shoulder/higher part of the back region could report pain in the neck, pain in the shoulder, and pain in the higher part of the back), some participants had more than one pain rating for a particular anatomic region. In those cases we used the highest pain rating within that anatomic region as the pain intensity measure for that particular anatomic region. For every anatomic region, screenpositives also had to indicate whether they currently used pain medication or had used pain medication during the past 12 months, whether they had contacted one or more specialists for their pain complaints during the past 12 months, and whether they had been on sick leave because of their pain during the past 12 months (absenteeism). The question about the use of medication was dichotomous (no $=0$, yes $=1$ ), the questions about specialist consultation and absenteeism were dichotomized (no $=0$, yes $=1)^{11}$. Chronicity was operationally defined as hawing pain with a duration of more than three months. For this purpose the data on pain duration were dichotomized as well (acute/subacute pain $=0$, chronic pain $=1$ ). Furthermore, the data of a standardized questiomaire that was included in the survey questionnaire were analyzed in the current study: the Dutch version of the PCS. This will be discussed into more detail below. The second survey questionnaire at follow-up six months later was similar to the first questionnaire except that the sociodemographic questions, the health-related questions referring to other (musculoskeletal) complaints and disorders than those referting to the five anatomic areas, and the PCS were omitted. Another difference was that the time period referred to in the questions about specialist consultation, use of pain medication, and absenteeism was changed (the past six months" in the follow-up questionnaire instead of "the past 12 months" in the baseline questionnaire).

\section{Pain catastrophizing}

The Dutch version (G. Crombez and J.W.S. Vlayen, 1996. Unpublished Dutch/folemish translation) of the PCS (Sullivan et al., 1995) was used. This is a 13-item scale in which

\footnotetext{
"In answering the question about speciallist consulation participants had to check when they had consulked a specialist for their complaints. If they had consulted a spectalist, they thad to theck one or more of 8 possibilities reflecting the specitic specialty. Because we wanted to know whether or not they had consulted a specialist and were not interested in what specific specialty they consulted, we dichotomixed this variable. The variable absenterism (ranging from "never" to "more than 4 weeks") was dichotomized ass well.
} 
participants are asked to reflect on past painful experiences and indicate the degree to which they experience thoughts or feelings during pain which are characterized by catastrophizing on a 5-point scale, ranging from 0 (not all all) to 4 (always). Examples of PCS items are: [when J'm in pain] "I keep thinking about how much it hurts" or "I wonder whether something serious might happen". Psychometric properties of the Dutch version of the PCS are adequate (Severeins al., 2002; Van Damme al., 2002a, Van Damme et all "2000).

\section{Statistical analysulis}

SPSS for Windows, Release 10.1.4 (SPSS Inc., Chicago, Illinois, USA) was used for all statistical analyses.

Logistic regression analyses were performed to examine prospective associations between pain catastrophizing (PCS) and chronicity and several other pain outcomes/health indices (i.e., specialist consultation, use of pain medication, and absenteeism). Because PCS scores. deviated from normality ( skewness $=1.29$, standard error of skewness $=0.056$ ) we performed a square root transformation on the PCS total scores. The degree of skewness posttransformation was 0.016 , which indicates that the distribution of the transformed PCS scores did not deviate from normality. The transformed PCS scores were entered as an independent variable in the subsequent regression analyses.

Overall, the participants who apart from the baseline questionnaire also completed and returned the follow-up questionnaire $(N=1888$ ) can be subdivided into three pain subgroups, based on whether they currently, that is, at baseline, experienced nusculoskeletal pain or not: (1) those without musculoskeletal pain, neither at baseline, nor in the year preceding the baseline inquiry (i.e., the group without pain, $n=424,22.4 \%$ ), (1) those without nusculoskeletal pain at baseline but with musculoskeletal pain in the year preceding the baseline inquiry (i.e., the group with recent pain, $n=345,18.3 \%$ ), and (3) those with musculoskeletal pain at baseline (i.e., the group with current musculoskeletal pain, $n=1119$, $59.3 \%)$. The group without pain was left out of the regression analyses.

The independent contribution of catastrophizing to the prediction of the outcome variables was tested for the group with current pain and the group with recent pain combined. Furthermore, two series of logistic regression analyses were performed in which the hypothesis was tested that the relationship between pain catastrophizing measured at baseline and chronicity and the health indices measured at follow-up is moderated by the factor pain, which is expressed as the number of pain regions endorsed and the pain intensity:

1) In the first series it was examined whether the relationship between pain calastrophizing and the dependent variables was moderated by the number of pain regions endorsed at baseline. The analyses were performed for the subgroup with current pain and the subgroup with recent pain combined. The number of pain regions endorsed could range from one to five.

2) In the second series it was examined whether the relationship between pain catastrophizing and the dependent variables was moderated by pain intensity at baseline. For this purpose the analyses were limited to participants with current pain at baseline..

All analyses were adjusted for age and sex. In testing the interaction between catastrophizing and pain, the covariates were entered as a block first, together with pain catastrophizing and the moderator main variable. The interaction terms were entered second. Each analysis was limited to those participants who did not endorse the particular dependent variable examined in that analysis at baseline. 


\section{Results}

\section{Descriptive results}

The mean PCS total scores for the subgroups without pain, with recent pain; and win current pain $(7.88,8.44$, and 10.95 respectively) significantly differed from each other (one way $A N O V A, F(2,1885)=23.80, p<.01$, effect size $=.025)$. Post hoc multiple (Tambane $T 2$ ) comparisons showed that the subgroup with current musculoskeletal pain catastrophized more about pain than the other two subgroups did.

Table 1 shows the PCS mean and median scores, standard deviations, and minimum and maximum scores for the group as a whole and the three pain subgroups (i.e., without pain, with recent pain, and with current pain).

Table 1 .

Mean, median, standard deviation, and minimum and maximum score of the Pain Catastrophizing Soale (PCS) for the sample as a whole and for the three different pain subgroups

\begin{tabular}{|c|c|c|c|c|}
\hline \multirow[b]{2}{*}{ Pain group } & \multicolumn{4}{|c|}{$\mathrm{PCS}$} \\
\hline & Mean & Median & Standard deviation & $\begin{array}{l}\text { Winimun-maximum } \\
\text { PCS sore }\end{array}$ \\
\hline Total group $(N=1888)$ & 9.80 & 8.00 & 8.90 & $0-52$ \\
\hline No pain $(y=424)$ & 7.88 & 6.00 & 8.00 & $0-40$ \\
\hline Recent pain $(F=345)$ & 8.44 & 7.00 & 7.38 & 0.44 \\
\hline Current pain $(n=1119)$ & 10.95 & 9,00 & 9.46 & $0-52$ \\
\hline
\end{tabular}

Nore. $N, n=$ mumber of cases.

Of the participants who reported musculoskeletal pain, either with current or with recent pain $(n=1464), 537(39.1 \%)$ reported pain in one anatomic area (mean PCS score $=9.48, S D$ $=8.43), 457(31.2 \%)$ reported pain in two anatomic areas (mean PCS score $=9.38, S D=$ $8.33), 270(18.4 \%)$ in three anatomic areas (mean PCS score $=11.20, S D=9.17), 110(7.5 \%)$ in 4 anatomic areas (mean PCS score $=14.60, S D=11.48)$, and $54(3.7 \%$ ) participants reported pain in all five anatomic areas (mean PCS score $=15.11, S D=11.37$ ). Participants who reported pain in more than one anatomic region catastrophized more about pain than participants who reported pain in only one anatomic region $(r(1315)=3.07, p<.01)$.

Of the participants with current pain $(n=1119), 81.6 \%(n=913)$ had chronic complaints (i.e., with a duration of more than three months). The mean pain intensity level of this group was 4.74 (minimum score $=1$, maximum score $=10, S D=2.35$, median $=5.00$ ),

Finally, of the group as a whole $(N=1888)$, participants who reported a higher level of baseline pain intensity catastrophized more about pain $(r=.28, p<.001)$.

\section{Prospective associations}

The logistic regression analyses yield odds ratios (OR) which indicate the relative amount by which the odds for having developed chronic complaints, having consulted a specialist, having been absent from work, and having used or using pain medication increase (OR>1) or decrease $(O R<1)$ when the value of pain catastrophizing is increased by 1 unit.

Table 2 shows the results of the first series of logistic hierarchical regression analyses in which the independent contribution of pain catastrophizing to the prediction of the dependent variables was tested. The results in table 2 indicate that the odds for development of chronic pain complaints increased for participants who catastrophize about their pain. No significant main effects of pain catastrophizing were found with respect to the health indices. There was 
a significant effect of sex on the use of pain medication, indicating that the odds for using pain medication decreased for women in comparison to men.

Table 2 .

Resulis of the logistic regression analyses for the group with current baseline pain and the group with recent baseline pain combined, whty catastrophizing as the independent variable and chronicity and the health indices at follow up as dependientin viriables

\begin{tabular}{|c|c|c|c|c|c|c|c|c|}
\hline \multirow[b]{3}{*}{ Nariable } & \multicolumn{8}{|c|}{ Dependent variables } \\
\hline & \multicolumn{2}{|c|}{$\begin{array}{l}\text { Chronicly } \\
(n-49)\end{array}$} & \multicolumn{2}{|c|}{$\begin{array}{l}\text { Speciallist } \\
\text { consultation } \\
(n=782)\end{array}$} & \multicolumn{2}{|c|}{$\begin{array}{l}\text { Use ar pain } \\
\text { medication } \\
(n=840)\end{array}$} & \multicolumn{2}{|c|}{$\begin{array}{c}\text { Absenteeism } \\
(n=541)\end{array}$} \\
\hline & $\mathrm{OR}$ & $95 \% \mathrm{CI}$ & $\mathrm{OR}$ & $95 \% \mathrm{CI}$ & OR & $95 \% \mathrm{Cl}$ & OR & $95 \% \mathrm{CI}$ \\
\hline A.ge & 1.01 & 0.99 .1 .03 & 1.01 & $1.00,1.02$ & 0.98 & $0.95,1.02$ & 0.99 & $0.97,1.01$ \\
\hline sex & 0.75 & $0.49,1.15$ & 0.99 & $0.68,1.45$ & $0.21^{*}$ & $0.06,0.78$ & 0.97 & $0.57,1.65$ \\
\hline Catastrophizing & 1. $\mathrm{1}^{\mathrm{in}}$ & $1.02,1.37$ & 1.06 & $0.93,1.21$ & 0.93 & $0.67,1.28$ & 1.06 & 0.88 .1 .29 \\
\hline
\end{tabular}

Nore. All analyses were aljusted for age and sex. $=$ number of cases included in the analysis; $\mathrm{OR}=$ odds ratio: CI wonfideruce interval.

$p<.05$

The results of the second series of logistic hierarchical regression analyses in which the interaction between pain catastrophizing and number of pain regions endorsed and pain intensity are examined, are shown in Tables 3 and 4 respectively.

Table 3 shows that in step 1 of the analyses there were significant main effects of the number of pain regions endorsed, indicating that participants with more pain problems were more likely to develop chronic complaints over a six months period and had a higher incidence of absenteeism. None of the interaction terms (pain catastrophizing $x$ number of pain regions) reached significance. Finally, table 4 shows that in step 1 of the analyses. participants who reported a higher level of pain intensity had a higher incidence of specialist consultation $(O R=1.14)$, use of medication $(O R=1.12)$, and absenteeism $(O R=1.17)$. None of the interaction terms (pain catastrophizing $\times$ pain intensity) reached significance.

\section{Discussion}

The primary aim of this study was to examine in a large Dutch community sample whether pain catastrophizing predicted the development of chronic pain, specialist consultation, the use of pain medication, and absenteeism. A secondary aim was to examine whether these relationships were moderated by the number of pain regions endorsed and by pain intensity.

With respect to the furst aim, we found a small effect of catastrophizing on the development of chronic musculoskeletal pain over a six months period. No evidence was found that pain catastrophizing predicted specialist consultation, the use of pain medication, or absenteeism.

With respect to the second aim of this study, we did not find any evidence that the relationship between pain catastrophizing on the one hand and chronicity and the health indices on the other hand was moderated by the number of pain regions and pain intensity.

These feeble results are rather surprising given the fact that the prevailing notion, based on contemporary catastrophizing research, is that catastrophizing plays a significant role in (chronic) pain. How then can the findings of the current study be understood?

One possible explanation is the low level of pain catastrophizing in the population sample used in the current study. The mean PCS scores for participants with current and with recent 

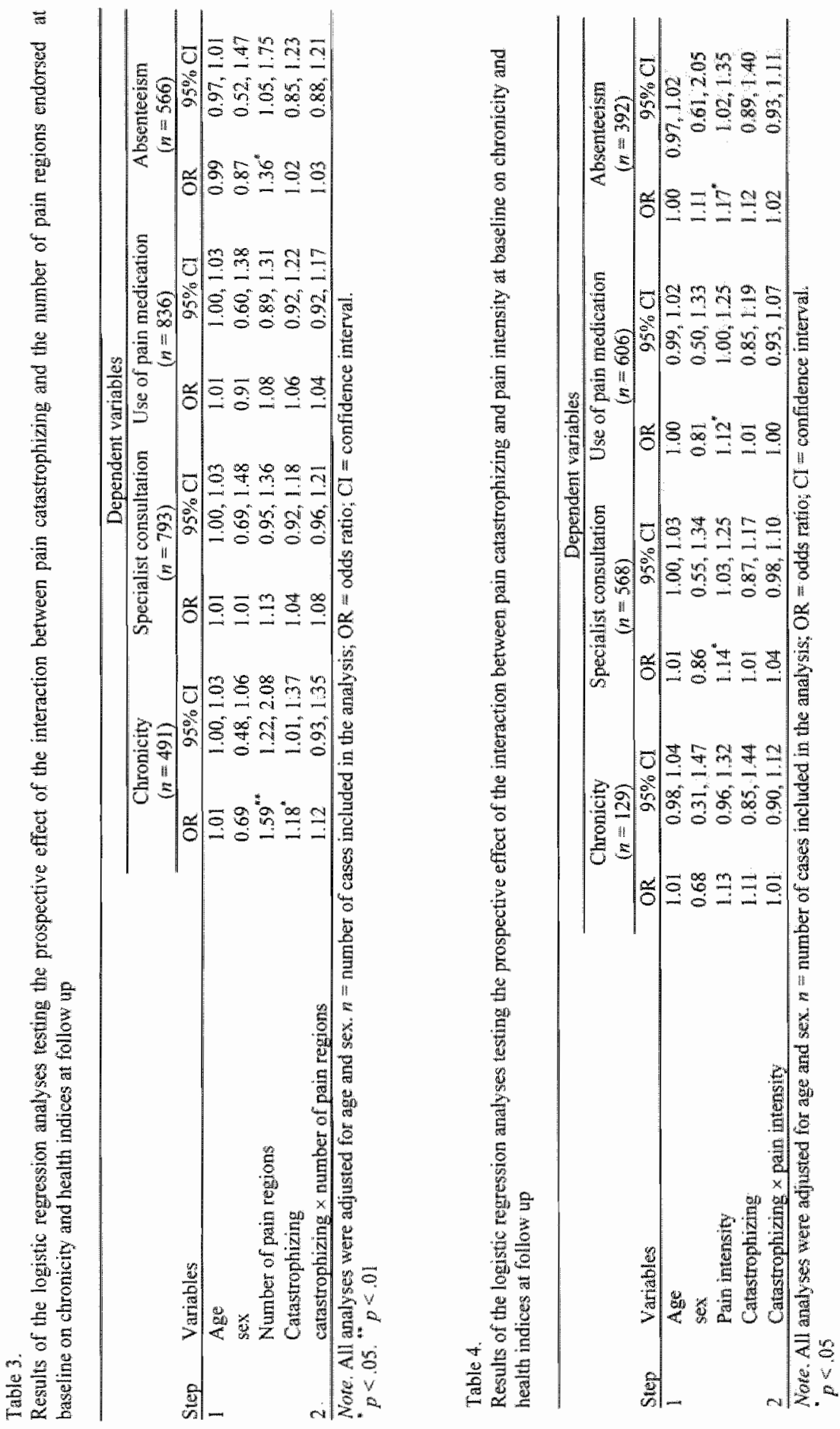
pain were 10.95 and 8.44 , respectively; the maximum PCS score possible was 52. For comparison, Osman et al. (2000) found a mean PCS score of 13.87 in a community sample as compared to a mean PCS score of 22.25 in an outpatient sample. In a psychometric study (Van Damme et all., 2000) mean PCS scores of 24.81 and 21.99 were found in a sample of fibromyalgia patients and patients with chronic low back pain, respectively. These examples demonstrate that the level of pain catastrophizing is notably lower in general community samples than in patient samples. This may very well be the rule rather than the exception and may well have contributed to the poor results. More specifically, despite the fact that positive associations were found between catastrophizing on the one hand and number of pain complaints and pain intensity on the other hand, and despite the moderate levels of pain intensity and the high prevalence of chromic pain complaints (over $80 \%$ of the participants with curtent pain had chronic complaints), the low levels of catastrophizing may well have prevented people from perceiving pain as very threatening.

Another possible, although perhaps less likely explanation is that there is a third variable that is both related to pain catastrophizing and the health indices and affects the relationship between pain catastrophizing and these health indices in time (i.e., prospectively), rather than at the same point in time (i.e., cross-sectionally). Neuroticism or negative affect is a qualifying variable in this respect that can be illustrated with a number of studies. Very recently, Goubert, Crombez, and Van Damme 2004) found that neuroticism moderated the relationship between pain catastrophizing and pain intensity. Also, neuroticism was shown to be significantly correlated to pain catastrophizing (see e.g., Affleck et al., 1992; Drossman et al., 2000; Sullivan et al., 1995). Finally, there are prospective studies in which neuroticism was identified as an independent risk factor for early postoperative pain after laparoscopic cholecystectomy (Bisgaard, Klarskov, Rosenberg \& Kehlet, 2001) and as a predictor of poor outcome at 1 year in patients with whiplash (Radanov \& Sturzenegger, 1996).

A third possibility is that the role of pain catastrophizing in predicting pain outcome variables is of minor importance, at least in the general population. In this respect it should be noted that the results of prospective studies on the role of pain catastrophizing in (chronic) pain are not unequivocal. In addition to prospective studies that found positive results for catastrophizing (Burton et al., 1995; Drossman et al., 2000; Keefe et al, 1989; H. S. Picavet ef al., 2002), there are studies that either found mixed results (Burns et al., 2003a; Burns et al., 2003b; Haythornthwaite et al., 2003; Jensen et al., 2001; Linton et al., 2000) or failed to find signiflicant effects of catastrophizing at all (Groth Marnat \& Fletcher, 2000; Pfingsten et al., 1997).

All together, the results of the current study may be somewhat difficult to interpret. Nevertheless, some considerations may be appropriate in this respect. For example, it should be remenbered that compared to the study of Picavet et al. (2002) which used the same dataset and found positive effects of catastrophizing, we used a more stringent method. That is, we used PCS continuous scores in stead of tertile scores and limited analyses to participants who did not have chronic pain at baseline. The small effect of catastrophizing on the development of chronic pain complaints might therefore be quite relevant, all the more because the PCS scores in the current sample were quite low. Another point is that although significant associations between pain catastrophizing and health indices such as use of pain medication and frequency of specialist consultation have been reported, little is known about the association between pain catastrophizing and these health indices in the natural environment of the individual (Sullivan et al., 2001b). Sullivan et al. (2001b) suggest the possibillity that the help-seeking context of clinical settings leads to an exaggerated display of behaviors such as use of medication or wisits to health care professionals. Alternatively, he proposes that the associations found so far between pain catastrophizing and these behaviors are actually an underestimation of the true relationship between catastrophizing and these 
behaviors because clinical and experimental settings lack the social cues that mormally trigger these behaviors. In the study of Severeijns et al. (2004) in which the same data set was used, the presence of these associations in the general community was confirmed cross-sectionally. This suggests that clinical/experimental settings and the natural environment (i.e., the general community) might be quite similar in this respect. However, in the current study examination of the prospective associations between pain catastrophizing and specialist consultation, use of pain medication, and absenteeism did not confirm these crossusectional results. Furthermore, the results do not corroborate the alternative hypothesis of Sullivan et al. (2001b) mentioned previously, which implies that in the natural enviroment the associations between catastrophizing and health indices such as specialist visits and use of pain medication should be more pronounced than in clinical or experimental settings because the latter lack the social cues that usually reinforce these behaviors. Clearly, more research on the possible social and contextual determinants of catastrophizing and these pain outcomes is needed.

Some critical remarks have to be made regarding the current study. First, because a mail-out method was used we did not have control over the way participants completed the questionnaire. Second, the follow-up period was relatively short which may have weakened the longitudinal power of the design and may account for the poor results. Third, follow-up data of only 1888 of the original 3664 participants who returned the baseline questionnaire were available for the current study $(51,5 \%)$. This means that some kind of selection bias can not be excluded which may also account for the different results of the cross-sectional study (Severeijns et al., 2004) and the current prospective study. More specifically, although the same dataset was used, the sample in the prospective study is not quite the same as the sample in the cross-sectional study because the former comprises the participants who had consented to be reapproached for the follow-up and in addition took the trouble to complete and return the follow-up questionnaire. The latter sample on the other hand also comprised a considerable number of people who refused to be reapproached for the follow-up or who consented to be reapproached but did not return the questionnaire. This may mean that different processes could play a part in the cross-sectional and the prospective sample. Fourth, although there is ample evidence that catastrophizing and depression are separate constructs, they are rellated (Geisser et al., 1994a; Geisser et all., 1994b; Haaga, 1992; Keefe et al., 1989 ; Keefe et al., 2000; Sullivan et 21., 1995; Sullivan et al., 2001a; Sullivan et al., 1998; Turner et al., 2000). Therefore, it might have been appropriate if depression had been included as a covariate in the analyses. Fifth, all the measures used in this study are self-report measures which might be subject to several kinds of bias.

In summarizing the above, some tentative conclusions can be drawn. We set out this study to examine whether catastrophizing is involved in the development of future pain related problems in the general community. Although pain catastrophizing plays a small role in predicting the development of chronic musculoskeletal pain, the low level of catastrophizing in the general community probably limits its influence considerably. Because in the general community a low level of catastrophizing is likely to be the rule rather than the exception, the role of catastrophizing in the development of future pain-related problems may be limited there as compared to its role in clinical settings. Apparently, the role of pain catastrophizing in (chronic) pain is very complex and not self-evident and the type of setting is important in this respect. Another, more practical implication is that, at least in the general community, there may be only limited use of the PCS as a screening instrument for detecting who is at risk of developing various health related problems in the near future. 


\section{CHAPTER 8}

The causal status of pain catastrophizing: An experimental test with healthy participants

This chapter will be published as:

Severeijns, R., van den Hout, M.A., \& Vlaeyen, J.W.S. The causal status of pain catastrophizing: an experimental test with healthy participants. European Jowmal of Pain (in press) 


\begin{abstract}
In the current study we report findings on the effects of experimentally induced catastrophizirig about pain on expected pain, experienced pain and escape/avoidance behavior during a cold pressor task in a sample of healthy participants. It was hypothesized that increasing the level of catastrophizing would result in a higher level of expected pain, a higher lewel of experienced pain, and a shorter duration of ice-water immersion. Also, it was hypothesized that these relationships might be stronger for participants who already catastrophized about pain prior to the experiment.

The results demonstrated that despite the successful attempt to induce catastrophizing, this neither significantly affected expected pain, experienced pain, and duration of ice-water immersion, nor were these relationships moderated by the pre-experimental level of catastrophizing.

Although the level of catastrophizing was successfully manipulated, more similar experiments are necessary in order to give a more definite answer on the possible causal status of pain calastrophizing.
\end{abstract}




\section{Introduction}

Cognitive-behavioral conceptualizations of how pain becomes persistent and chronic have received increasing attention in recent years. This is not surprising because the relationship between physical impairment, pain and disability is only modest at best (Feuerstein et al., 1987; Vlaeyen et al., 1995b; Waddell, 1987; Waddell \& Main, 1984; Waddell ett al, 1993; Waddell et al., 1989). Several authors proposed that cognitive and/or behaviorall factors play a causal role in the development of pain-related disability, avoidance, deconditioning, depression etc. (e.g, Lethem et al, 1983; Philips, 1987; Reesor \& Craig, 1988; Turk, 19966; Turk \& Femandez, 199!; Turk \& Rudy, 1992; Vlaeyen et al., 1995a; Vlaeyen et al., 1995b). One of these factors is pain catastrophizing that involves an extremely and exaggeratedly negative orientation towards pain itself and its possible consequences (Sullivan et al., 1995). There is ample evidence from several recent studies that pain catastrophizing is consistently related to pain outcome variables such as disability, psychological distress, depression, and pain (for an extensive owerview see Sullivan et al., 2001 b).

Nevertheless, most of the research on pain catastrophizing is cross-sectional, and the results are correlational which make statements on the causal nature of the relationship between catastrophizing and pain outcome variables (e.g. disability, psychological distress, and pain severity) rather hypothetical. On logical grounds catastrophizing might also result from pain-related disability, psychological distress, and pain severity. A prospective study of Burton et al. (1995) suggests that catastrophizing may, indeed, play a causal role. That is, it was shown that for patients with a current episode of low back pain (with a duration of three weeks or less) catastrophizing was the most powerful predictor of pain chronicity at one year. Furthermore, in an experimental study with healthy participants (Sullivan et al., 1995), it was demonstrated that catastrophizing, measured approximately six weeks prior to testing, successfully predicted pain in a cold pressor test, even when controlling for other variables such as fear of pain, negative and positive affect, depression, state and trait anxiety. Although these studies show that catastrophizing is a precursor of (chronic) pain and pain intensity rather than the other way around, a more direct demonstration of the causal role of catastrophizing is still lacking. That is, while the association between catastrophizing and (chronic) pain can apparently not be explained by pain causing catastrophizing, it remains possible that the catastrophizing-pain association is due to some third variable, not controlled for in earlier studies. In this respect, the successful experimental manipulation of catastrophizing should yield the needed information about the causal role of catastrophizing in (chronic) pain. More specifically, the current experiment will critically test the still hypothetical causal relationship between catastrophizing and pain intensity. Furthemone, knowing more about the possible causal role of pain catastrophizing is important because catastrophizing is a construct that has proven to be very relevant in the study of pain and plays a prominent role in the fear-avoidance model of chronic pain (Vlaeyen et al, 1995 a; Viaeyen et al., 1995b) which is one of the prevailing cognitively oriented models on (chronic) pain. The model states that catastrophizing about pain, via its influence on fear of movement/(re)injury, promotes avoidance, disuse, depression, disability, and the development of chronic pain.

A comparable experiment in the field of anxiety/fear was conducted by Salkowskis and Clark (1990). In order to test the cognitive model of panic, the interpretation of the bodily sensations associated with voluntary hyperventilation were experimentally manipulated. For this purpose a catastrophic misinterpretation of these sensations was induced in the experimental condition and a positive interpretation was induced in the control condition. As predicted, Salkovskis and Clark (1990) found that participants in the experimental condition experienced hyperventilation as unpleasant while participants in the control condition 
experienced hyperventilation as pleasant. These results were interpreted as providing support for the cognitive model of panic which states that the extent to which innocuous sensations are interpreted in a negative/catastrophic way is a major determinant of panic (Salkovskis \& Clark, 1990).

Similarly, the aim of the current study was to examine the effects of experimental manipulations of catastrophizing on subjectively reported pain intensity and on actual behavior. An attempt was made to temporarily make heallhy participants catastrophize about pain. It was hypothesized that temporarily inducing catastrophizing would increase the level of expected pain, maximun experienced pain, and promotes avoidance/escape behavior in a cold pressor task, the latter reflected in a significantly shorter duration of the ice-water immersion. Also, we hypothesized that participants who already catastrophized about pain prior to the experiment are particularly sensitive to the catastrophizing induction and that the effects of the catastrophizing induction on the dependent variables are more pronounced for these participants.

Participants were randomly allocated to two groups. In the experimental group, the level of catastrophizing was experimentally induced. We expected that any increase in the level of catastrophizing would be accompanied by a non-specific increase in negative mood, which theoretically might also explain any effects on the dependent variables. To check whether any effects in the experimental group were due to the specific information given, i.e. catastrophizing instructions, and were not simply the result of an increased negative mood, participants in the control group were given a generally negative and threatening message, aimed at inducing a general negative mood. Manipulation checks were used to verify if the experimental manipulations and the negative mood inductions were indeed successful. It was expected that a negative mood increase would occur in both groups (in the experimental group indirectly, as a result of the catastrophizing induction).

\section{Method}

\section{Participants}

From other studies with a cold pressor task (see e.g., van den Hout, Vlaeyen, Peters, Engelhard \& van den Hout, 2000) it is known that the inter-individual variation of the immersion duration is quite large. Furthermore, the catastrophizing induction was expected to have only small effects. For this reason a power analysis was conducted from which the number of participants needed in each group was estimated to be approximately 35. Participants were partly recruited from an existing pool of people who had participated in studies before (none of the participants had ever participated in a cold pressor task) and partly reeruited in a roundabout way (i.e., relatives and acquaintances of colleagues were asked to participate). They were contacted by telephone and were asked if they were interested to participate in an experiment in which the relationship between physical discomfort and mood was to be examined. Those who were interested were sent an information form, containing general information about the experiment, a form consisting of some questions concerning demographic data, health status, hand dominance, the Pain Catastrophizing Scale (PCS), and an informed consent form. After they had completed the questionnaires, signed the informed consent form, and returned the forms, they were contacted again to make an appointment for the experiment. Participants were randomly allocated to the experimental or the control group following an alternating scheme.

Participants between 18 and 65 years old were included for this study. Participants were excluded if suffering from any identified and serious medical condition (e.g., a serious hart condition). 
A total of 72 persons were found willing and suitable to participate in the current sidy. Of these $57(79.2 \%)$ were women (mean age $=47.4$ years, $S D=12.7$, range $=46.4$ years) and 15 (20.8\%) were men (mean age $=55.2$ years, $S D=8.8$, range $=32.8$ years). Most of he participants were community adults.

\section{Measures}

\section{Pain intensity}

In this study Numerical Rating Scales (NRS) (Jensen \& Karoly, 1992) were used to meastre maximum expected pain during the icewater immersion, experienced pain furing the loe water immersion at 30 seconds intervals, and maximum experienced pain during the ice =water immersion. Participants were asked to indicate with a number on a scale ranging from 0 (no pain) to 100 (worst pain ever) how much pain they maximally expected and the maximum pain they actually experienced during the ice-water immersion. Also, during the lce-water immersion, participants were given an audible signal every 30 seconds. At this signal they were instructed to verbally rate their current pain intensity on a scalle ranging from 0 (no pain) to 100 (wors: pain ever). The upper end of the NRS (100) was anchored for each participant by asking him or her to imagine that particular situation in which they had experienced the worst pain ever. The lower end of the NRS (0) was anchored by asking participants to imagine a situation in which they were pain free (this could also be the current situation).

\section{Pain catastrophizing}

The Dutch version (an umpublished, 1996 translation by G. Crombez and J.W.S. Vlaeyen) of the Pain Catastrophizing Scale (PCS) (Sullivan et al., 1995) was used. This is a 13-item scale in which participants are asked to reflect on past painful experiences and indicate the degree to which they experienced thoughts or feelings during pain which are characterized by catastrophizing on a five-point scale, ranging from 0 (not at all) to 4 (always). Psychometric properties of the Dutch version of the PCS are adequate: it correlates highly $\left(r^{*}=0.73\right)$ with the catastrophizing subscale of the Dutch Pain Cognition List (PCL) (Vlaeyen et al., 1990), has a good temporal stability (Pearsons $r=0.92$ ) and a high internal consistency (Cronbach"s alpha $=0.85$ ) (Crombez et al., 1998b). The internal consistency of the PCS in the current sample is high as well (Cronbach's alpha $=0.92$ ). Furthermore, the PCS appears to measure the same processes in both healthy volunteers as in clinical populations, as well as in community samples (Severeijns et al., 2002; Van Damme et al., 2002a).

\section{Momipulation checks}

The PCS manipulation check (PCS-me) was used in this study as a manipulation check for the catastrophizing manipulation in the experimental group. It consists of seven items, some of which are adapted from the original PCS and some of which are new (see appendix), and participants were asked to indicate to what extent every statement applies to them on a fivepoint scale, ranging from 0 (not at all) to 4 (very much)(see appendix). A "moment" time frame was used i.e., participants were asked to reflect on the seven items "as it is now, at this very moment". The internal consistency of the PCS-mo in the current sample was high (Cronbach's alphat $=0.85$ ).

The Positive And Negative Affect Scales (PANAS) (Watson, Clark \& Tellegen, 1988) were used as a check for the negative mood induction in the control group and to warrant the credibility of the alleged research question as it was put to the participants (i.e., to study the relationship between physical discomfort and mood). It consists of 20 descriptors of positive and negative feelings and emotions which had to be rated by participants on a five-point scale, ranging from 1 (very slightly or not at all) to 5 (extremely). The "moment" time instruction was used ("you feel this way right now, that is, at the current moment"). 


\section{Crediblity of he manipulation instrwetion}

The credibility of the manipulation instruction in the experimental and control group was measured with a NRS. After the ice-water immersion, just before the debriefing, participants were instructed to indicate with a number ranging from 0 (not at all) to 100 (absolutely) how credible the instruction as given to them by the experimenter was.

\section{Dummy questionnaire}

A dummy questionnaire was developed especially for this study, consisting of 15 fake guestions purported to measure "psychological stability" and "emotional competence". Participants were asked to indicate to what extent every item applied to them on a five-point scale, ranging from 1 (not at all) to 5 (very much). Example items are: "I am generally eventempered", "other" people see me as a balanced person", and "I attach great importance to my feelings". This questionnaire was included to allow for a credible instruction in the control group, where participants were informed that close inspection of this questionnaire revealed seme disturbing information about their psychologicall stability and emotional competence. The purpose of this instruction in the control group was to induce a general negative state of mind and to control for the possible non-specific effects of the catastrophizing induction in the experimental group.

\section{Behavioural test \\ Appararus}

The cold pressor apparatus consisted of a tempex container filled with ice-water, circulated by a fish-tank aerator. The temperature of the ice-water was held constant at $0^{\circ}-2^{\circ} \mathrm{C}$. This was ascertained by a thermometer and by periodically adding ice to the container. The ice was removed from the container and the water temperature was recorded for each participant before actual ice-water immersion. The water temperature could not be read by the participant. The duration of the ice-water immersion was recorded with a stopwatch. The stopwatch was kept out of sight to avoid the suggestion that the time of immersion was important. Participants were instructed to immerse their non-dominant hand up to their wrist in the ice-water. Directly preceding the ice-water immersion participants were asked to immerse their non-dominant hand for half a minute in a tempex container with water of room temperature to promote the similarity of hand temperature at the start of the ice-water immersion.

\section{Procedure}

On arrival, participants were told that the study was investigating the relationship between physical discomfort and mood. It was explained to them that for this purpose they would be asked later on to immerse their non-dominant arm up to their wrist in a container containing ice-water which would give an unpleasant physical sensation. Participants then, after instruction, completed the NRS expected pain, the PCS-mc, the PANAS, and the dummy questionnaire. Subsequently, participants were given a half an hour break during which they were allowed to watch a documentary ("Gilass" by Bert Haanstra). This was done for several reasons. First, to permit some time between the first measurement and the second measurement which was just before the ice-water immersion. Second, in both the experimental and control instructions participants were informed that the questionnaires they had completed at their arrival had been inspected by a psychologist during the half an hour brake (see bellow).

Next, the immersion procedure was introduced and explained. Participants were randomly assigned to one of two groups. Instructions in both groups were identical, except for a 
sentence in which a catastrophic interpretation of the sensations during the ico-water immersion was provided in the experimental group, and a sentence in which a general negative state of mind was induced in the control group. "Thus, all participants were told that:

"this experiment investigates the relationship between physical disconfort and nood. In moment you will be asked to immerse your non-dominant hand up to your wrist into the icewater container for as long as you do not feel too uncomfortable. Most people during the icewater immersion experience tingling, prickling, numb, and mildly painful sensations, sometimes evolving into a sharp and cutting pain. In general these sensations are quite harmless. During the ice-water immersion, you will be asked every 30 seconds bow much pain you experience at that moment. For this purpose you will be presented with an audible signal every 30 seconds (this part of the instruction was followed by a demonstration of the audible signal). When you hear this signal, I would like you to report a number between 0 and 100 that indicates how much pain you have at that moment. With this 0 means that you have no pain at all and 100 means worst pain ever. Is that clear?" For participants in the experimental group, the instructions continued:

"However, before we continue, we must inform you that a few people who have participated in the experiment have fainted during the ice-water immersion as they experienced sharp and cutting pain sensations. During the half an hour brake a psychologist closely inspected the questionnaires you completed at your arrival and this has revealed that your answers to the items of the questionnaires very closely resemble the answers of these people".

For participants in the control group the instructions continued:

"before we continue we must inform you that during the half an hour brake a psychologist closely inspected the questionnaires you completed at your arrival and this has revealed some disturbing information concerning your psychological stability and emotional competence. We therefore would like to ask you to stay after the experiment to talk with a psychologist about the possible action to take".

Subsequently participants were asked to complete the NRS scale for expected pain, the PCS$\mathrm{mc}_{\text {, }}$ and the PANAS. Next, all participants were instructed to immerse their non-dominant hand up to their wrist into the container with water of room temperature. After half a minute they were instructed to remove their hand and to immerse it in the container with ice-water for as long as they did not feel too uncomfortable. Every 30 seconds they completed a NRS current pain. The maximum allowed duration of the ice-water immersion was five minutes, after which they were asked to remove their arm from the ice-water and to rest it on a towel placed on their lap. Immediately following the ice-water immersion participants were asked to complete the PANAS and the NRS credibility. Finally, all participants were thonoughly debriefed. During the debriefing process participants were given detailed information about experimental research in general and the current study in particular. "They were informed about the actual research question and about the reason why the experiment was performed in this way. With respect to the dummy questionnaire, participants were informed that this is a fake questionnaire that does not bear any reference to psychological stability and emotional competence. Finally, they were given the opportunity to ask any questions they might still have.

\section{Statistical analysis}

The General Linear Model (GLM) with repeated measures procedure was used to examine whether the catastrophizing and the negative mood induction were successful. We expected a larger increase in the level of catastrophizing in the experimental group than in the contro! group. Also, we expected the level of negative mood to increase in both the experimental and the control group. 
The GLM with repeated measures was used to examine whether making participants catastrophize about pain (the experimental manipulation) resulted in a higher level of expected pain. Independent-samples $t$-tests were performed to examine whether the experimental manipulation resulted in a higher level of maximum experienced pain during the ice-water immersion and a shorter duration of the ice-water immersion.

The GLM with repeated measures (expected pain) and two separate ANOVA's (maximum experienced pain and duration of ice-water immersion) were performed to test our hypothesis that the effects of the catastrophizing induction on the dependent variables might be more pronounced for participants who already catastrophized about pain prior to the experiment (i.e., the interaction between "group" and catastrophizing (PCS score)).

\section{Results}

\section{Descriptives}

The level of catastrophizing as measured with the PCS prior to the experiment was quite low (maximum possible $\mathrm{PCS}$ score $=52)$ in both the experimental group $(M=10.84$, Minimum score $=0$, Maximum score $=36, S D=7.88)$ and the control group $(M=14.40$, Minimum score $=5$, Maximum score $=33, S D=7.55$ ). The level of catastrophizing prior to the experiment was higher in the control group compared to the experimental group $(t(70)=$ $-1.96, p=0.05$, two-tailed). The credibility of the instructions was high (maximum possible score $=100)$ in the experimental group $(M=85.83, S D=18.07)$ as well as in the control group $(M=87.79, S D=10.60)$. Participants in both groups did not differ on the level of credibility of the respective instructions $(f(67)=-0.55$, n.s., two-tailed). Nevertheless, in the experimental group there were two participants who reported a credibility score of only 10 and 40 respectively. These scores appeared to be outliers on this measure ${ }^{12}$.

\section{Manipullation check}

The GLM with repeated measures procedure was used to examine whether the catastrophizing induction was successful. "Group" (the experimental group or the control group) was entered as between-subjects factor and "time" (PCS-mc before and after instruction) as within-subjects factor. As expected, there was a significant "time $\times$ group" interaction effect $(F(1,70)=10.22, p<0.01)$. Pairwise comparisons revealed that in the experimental group there was a significant increase in the level of catastrophizing following the experimental (i.e., induction of catastrophizing) instruction $(t(36)=-3.39, p<0.005$, two-tailed). In the control group there was no significant change in the level of catastrophizing following the control (i.e., induction of negative mood) instruction $(r(34)=0.84$, n.s., two-tailed). This means that the catastrophizing induction in the experimental group was successful. Furthermore, the experimental group and the control group did not differ in the level of catastrophizing just before the catastrophizing induction (PCS-mc score time $1 ; r(70)=-1.00$, n.s., two-tailed), nor did they differ in the level of catastrophizing after the catastrophizing induction (PCS-mo score time $2, t(70)=1.17$, n.s., two-tailed). Descriptives for the change in catastrophizing in both groups are shown in Table 1.

Similarly, the GLM with repeated measurements procedure was used to examine whether the general negative state of mind induction was successful. "Group" (the experimental or the control group) was entered as between-subjects factor and "time" (the PANAS negative affect scores before and after instruction) as within-subjects factor. Contrary to what we expected, there was no significant effect for "time" $(F(1,70)=3.04$, n.s.). There was however a

\footnotetext{
12 Postwho analyses were performed with the outliers on the credibility measure in the experimental group removed from the data. However, this did not change the results of the analyses substantially.
} 
significant "time $\times$ group" interaction effect $(F(1,70)=4.00, p<0.05)$. Pair wise comparisons revealed that in the experimental group there was a significant increase in the level of negative mood following the experimental (i.e, induction of catastrophizing) instruction $(t(36)=-2.42, p<0.05$, two-tailed). In the control group there was no significant change in the level of negative mood following the control (i.e, induction of negative mood) instruction (v(34) $=0.21, \mathrm{n} . \mathrm{s}_{*}$ two-tailed). This means that the negative mood induction in the control group was not successful. Also, there were no differences between the experimental group and the control group in negative mood, neither before the negative mood induction (PANAS negative affect scores time $1, t(50.18)=-1.38$, n.s., two-tailed), nor after the negative mood induction (PANAS negative affect scores time $2, n(70)=0.07$, n.s., two-tailed. Descriptives for the change in negative mood in both groups are shown in Table 2.

Table 1.

descriptives for the catastrophizing induction

\begin{tabular}{lcccc}
\hline Condition & time & mean & Standard deviation & N \\
\cline { 5 - 5 } Experimental & 1 & 4.46 & 3.20 & 37 \\
& 2 & 6.16 & 4.48 & 37 \\
control & 1 & 5.37 & 4.49 & 35 \\
& 2 & 5.06 & 3.41 & 35 \\
\hline
\end{tabular}

Trable 2.

descriptives for the negalive affect induction

\begin{tabular}{lcccc}
\hline Condition & time & mean & Standard deviation & N \\
\hline Experimental & 1 & 11.78 & 2.51 & 37 \\
Control & 2 & 13.03 & 3.09 & 37 \\
& 1 & 13.06 & 4.87 & 35 \\
\hline
\end{tabular}

\section{Effects of inducing pain catastrophizing}

Descriptives for the dependent variables (expected pain, maximum experienced pain, and duration of ice-water immersion) are shown for both the experimental and control group in Table 3.

Table 3.

Descriptives for the dependent variables

\begin{tabular}{|c|c|c|c|c|c|c|c|c|}
\hline \multirow[b]{3}{*}{ Group } & \multicolumn{8}{|c|}{ Dependent kablebs } \\
\hline & \multicolumn{2}{|c|}{$\begin{array}{c}\text { Expected pain } \\
\text { time } 1\end{array}$} & \multicolumn{2}{|c|}{$\begin{array}{l}\text { Expecled pain } \\
\text { tinge } 2\end{array}$} & \multicolumn{2}{|c|}{$\begin{array}{c}\text { Maximuin } \\
\text { experienced pain }\end{array}$} & \multicolumn{2}{|c|}{$\begin{array}{l}\text { Irmmersion } \\
\text { dullation }\end{array}$} \\
\hline & Mean & $S D$ & Mean & 50 & Mean & $S D$ & Mean & $S D$ \\
\hline Experimental group & 40.00 & 21.76 & 46.22 & 22.12 & 75.27 & 20.98 & 13897 & 111.23 \\
\hline Control group & 40.97 & 19.63 & 43.77 & 23.04 & 72.63 & 25.20 & 99,46 & 90.98 \\
\hline
\end{tabular}

Nore. Expected pain time I refers to the amount of pain expected pritor to the expetimental or control instruction; expected pain time 2 refers to the anount of pain expected after the experimental or control instruction. 
The GLM with repeated mensures procedure was used to test the Hypothesis that making participants catastrophize about pain during the ice-water immersion would lead them to expect more pain. The results demonstrated that there was" a significant effect for "time" (expected pain before and after instruction) $(F(1,70)=8.94, p<0.01$ ) but the crucial interaction ("time $\times$ group") did not reach significance $(F(1,70)=1.31$, n.s.). This indicates that although overall participants expected more pain after instruction, participants who were made to catastrophize about pain did not expect more pain than participants in the control group:

Independent-samples $t$-tests were performed to test the hypotheses that participants who were made to catastrophize about pain during the ice-water immersion would experience a higlier maximum pain intensity and have a shorter duration of ice-water immersion than participants in the control group. The results demonstrated that there was no significant difference between the experimentall and the control group with respect to maximum experienced pain $(t(70)=0.48$, n.s., one-tailed). The effect with respect to the duration of icewater immersion $(70)=1.64, p=0.05$, one-tailed) was significant but in the opposite direction of what was expected. This means that both hypotheses were not confirmed.

The hypothesis that participants who already catastrophized about pain prior to the experiment would be particularly sensitive to the catastrophizing induction, was tested by examining whether there was an interaction effect between pain catastrophizing prior to the experiment and "group". Using the GLM with repeated measures, no significant "PCS $x$ group $\times$ time" interaction effect was found with respect to expected pain $(F(1,68)=0.28$, n.s.). Also, using ANOVA"s no significant "PCS $\times$ group" interaction effects were found with respect to maximum experienced pain $(F(1,68)=0.61$, n.s.) and duration of ice-water immersion $(F(1,68)=0.25$, n.s.). This means that the effects of the catastrophizing induction on expected pain, experienced pain, and duration of ice-water immersion, was not more pronounced for participants who already catastrophized prior to the experiment.

\section{Discussion}

To the best of our knowledge this is the first study in which an attempt was made to speciffcally manipulate the level of catastrophizing about pain and in which the effect of this manipulation on expected and experienced pain and escape/avoidance behavior during experimentally induced pain was examined. The study was designed to shed some more light on the question whether there is a eausal relationship between pain catastrophizing and various pain-related outcome variables such as pain intensity and avoidance behavior.

The results demonstrated that the attempt itself to manipulate the level of catastrophizing in the experimental group was successful contrary to the attempt to induce a general negative state of mind in the control group. In fact, in the experimental group there was an increase in negative affect scores while in the control group there was no significant change in negative mood at all. Apparently, the type of instruction and feedback we used to induce a negative mood was not adequate. A meta-analysis on the effectiveness of mood induction procedures demonstrated that for the induction of negative mood states, failure feedback on a test (usually "testing" cognitive abilities) was, amongst other procedures, nearly as successful as a film or a story (Westermann, Spies, Stahl \& Hesse, 1996) which was the most successful.

Nevertheless, the experiment illustrates that even with a relatively simple instruction the level of catastrophizing can be significantly manipulated and that increasing the level of catastrophizing appears to have a negative impact on mood. However, the increase in catastrophizing was only very modest which raises the question of clinical significance. The null findings in this experiment can not simply be equated with lack of clinical significance outside the experimental situation. It also raises the question whether it would be equally simple to reduce the level of catastrophizing in patients with (chronic) pain who catastrophize 
about their pain. A similar experiment using a patient sample could answer this question which has some clinical relevance as well.

Our primary hypothesis that making participants catastrophize about pain during a cold pressor task would lead them to expect more pain, to experience a higher maximum level of pain intensity during the ice-water immersion, and to have a shorter duration of ice-water immersion, was not confirmed.

There are several possible explanations for these results. First, we know from other cold pressor studies that the inter-individual variation of immersion duration as expressed in the standard deviation is quite large (see e.g., van den Hout et al, 2000). This is due to the fact that there are some people who are able to keep right on with the immersion to the maximum duration time set in advance. In the current study the maximum duration of immersion was set at 5 minutes. A closer look at the results demonstrated that 10 out of 13 participants with a maximum duration of immersion were in the experimental group. Furthermore, in general the experimental group had a significantly longer duration of ice-water immersion. Although bypothetically the increase in catastrophizing in this group may have resulted in a longer duration of immersion, this is not very likely. Alternatively, motivational factors and social desirability might be important in this respect. Perhaps the participants in the experimental group were highly motivated to convince the experimenter that they were able to hold on or they more or less assumed that this was expected of them. Another possibility is that the difference in the level of catastrophizing prior to the experiment between experinental and control group might have caused this. These factors then may have counteracted any possible effect of the catastrophizing induction.

Second, despite the fact that there was sufficient ground for performing the experiment the way we did (see for instance the comparable experiment by Salkovskis and Clark (1990)), the specific task in this experiment (ice-water immersion) in combination with the manipulation instruction might nevertheless have been too weak. Because the actual increase in pain catastrophizing in the experimental group was only modest, the instruction with which we attempted to induce the level of catastrophizing was perhaps overcautious, despite the fact that its credibility generally was quite high. Perhaps the instruction would have been more successful in inducing the level of catastrophizing if we had emphasized the negative consequence of ice-water immersion (i.e., fainting) more convincingly as something that wass quite likely to occur. Providing the participants with a plausible, though not necessarily valid, medical rationale for this negative consequence (e.g., fainting as a result of strong vasoconstriction due to the ice-water immersion) might have added to the effect.

Third, the pre-experimental level of catastrophizing was quite low, with a mean PCS score of 12.57 for the sample as a whole. This is closely in line with Severeins et al. (Severeinns et al., 2002) who found a mean PCS score of 10.04 in a community sample and with Osman et al. (2000) who found a mean PCS score of 13.87 in a community sample as compared to a mean PCS score of 22.25 in an outpatient sample. Also, mean PCS scores of 24.81 and 21.99 were found in a sample of fibromyalgia patients and chronic low back pain patients respectively (Van Damme et al, 2000). It is plausible to assume that due to the generally low pre-experimental level of catastrophizing in the current sample, participants were not particularly susceptible to any instruction aimed at inducing catastrophizing, no matter how credible that instruction might have been, and that it has prevented the expected moderation effect from being fully expressed. Consequently, the level of catastrophizing might have increased only slightly (as indeed was the case), and the actual increase might have been too small to be of significant influence on the dependent variables, although it apparently did have some effect. Another possibility is that the expected moderation effect was obscured by the fact that participants in the control group catastrophized significantly more prior to the experiment than participants in the experimental group or that the restricted range of 
catastrophizing scores may have hindered the sensitivity of the interaction analysis. Replication of this experiment with clinical/outpatient samples where there are higher levels of catastrophizing, and using a stronger experimental instruction is indicated. Furthermore, because the experimental and the control group significantly differed on the pre-experimental level of catastrophizing, it might have been advisable if both groups had been matched on this variable.

Finally, it is also possible that the null findings in this experiment are an indication that there is no causal relationship between catastrophizing and escape/avoidance behavior, experienced pain, and expected pain. It should be noted though that in the experimental group we attempted to remporarily inerease the level of catastrophizing by explicitly referring to painful sensations during the cold pressor task (and not to pain in general) and by using a "momentary" time-frame in the manipulation check (PCS-mc). This "state" catastrophizing may be quite different from catastrophizing as measured with the PCS which has more stable and trait-like qualities (see e.g., Sullivan et al., 200 lb). It is conceivable that this "irait" catastrophizing is the more crucial of the two in affecting expected pain, experienced pain, and duration of ice-water immersion in this experiment, but that we failed to influence it with our manipulation that was aimed at "state" catastrophizing.

Summarizing the foregoing, the main conclusion to be made is that the level of catastrophizing can be manipulated relatively easy although the current study does not give a definite answer to the question whether there is a causal relationship between catastrophizing and expected pain, experienced pain and avoidance/escape behavior in experimentally induced pain. This study might be the first in a series of similar experiments with clinical and/or outpatient samples in which the level of catastrophizing is experimentally induced or reduced.

Appendix A. The items of the catastrophizing manipulation check

To what extent:

Do you expect the ice-water immersion to cause pain.

Do you wonder whether something serious might happen.

Do you think that pain signifies "danger".

Do you believe that any increase in pain during the ice-water immersion will have some longer lasting negative consequences.

Are you afraid that the ice-water immersion might cause pain.

Are you thinking about how much it might hurt.

Are you thinking of other painful experiences. 


\section{CHAPTER 9}

The causal status of pain catastrophizing: An experimental test with chronic low back pain patients

This chapter is submitted as:

Severeijns, R., Vlaeyen, J.W.S. \& van den Hout, M.A. The causal status of pain catastrophizing: an experimental test with chronic low back pain patients. 


\begin{abstract}
In the current study we investigated the effects of experimentally reduced catastrophizing about pain on expected pair, experienced pain, and task performance during a KneeExtension-Flexion (KEF) task on a Biodex system 3 apparatus. It was hypothesized that reducing the level of catastrophizing would result in a lower level of expected pain, a lower level of experienced pain, and a better task performance on the KEF-task.

The results demonstrated that the attempt to experimentally reduce catastrophizing was effective with those participants who catastrophized about pain, that is, who had relatively high levels of catastrophizing about pain. Also, the group of participants whose level of catastrophizing was reduced, performed better on two KEF-task performance parameters than the group whose level of catastrophizing was not reduced. However, due to statistical power problems it could not be established that this better performance was directly related to the reduction in catastrophizing.
\end{abstract}




\section{Introduction}

Although there is still a lot of controversy about the exact nature of pain catastrophizing and how it should be conceptualized, evidence is cumulating that it is consistently irelated to pain outcome variables such as disability, psychological distress, depression, and pain severity (for an extensive overview see Sullivan et al $2001 \mathrm{~b}$ ).

Several cognitively oriented models have been developed in which cognitive variables, among which pain catastrophizing, are thought to foster the development of pain-related disability, avoidance, deconditioning, depression etcetera, e.g., the "fear-avoidance" model (Lethem et al, 1983), the "pain-avoidance" model (Philips, 1987), and more recently, the "fear of movement/(re)injury" model (Vlaeyen et al., 1995a; Vlaeyen et al., 1995b). Evidenoe for the causal role of pain catastrophizing in (chronic) pain however, is circunstantial and inconclusive because all of the studies we know of are cross-sectional or prospective at best. This of course makes statements on the causal nature of the relationship between catastrophizing and pain outcome variables (e.g., physical performance, disability, psychological distress, and pain severity) rather hypothetical.

Nevertheless, there are some prospective studies that suggest that pain catastrophizing is a precursor of (chronic) pain, pain intensity, and other pain-related variables rather than the other way around (e.g., Burton et al., 1995; Drossman et al., 2000; Jensen et al., 2001; Keefe ef al., 1989; Linton et al., 2000; H. S. Picavet et al., 2002; Sullivan et al., 2002). However, there is still the possibility that the catastrophizing-pain association is due to some third variable, not controlled for in earlier studies. Clearly; a more direct demonstration of the causal role of catastrophizing is needed. This could be achieved by examining the effects of the experimental manipulation of catastrophizing on pain-related outcome variables.

A comparable experiment in the field of anxiety/fear was conducted by Salkovskis and Clark. (1990) who experimentally manipulated the interpretation of the bodily sensations associated with voluntary hyperventilation in order to test the cognitive model of panic. In a sumilar experiment with healthy participants, we examined the effects of experimentally induced catastrophizing about pain during a cold pressor task (Severeijns, van den Hout \& Vlaeyen, in press). Although the attempt to induce catastrophizing was successful, we did not find any supportive evidence for the causal role of catastrophizing. However, catastrophizing scores before and after the experimental manipulation were quite low which may have prewented significant effects of catastrophizing on the dependent variables to occur.

Therefore, the aim of the current study was to further examine the effects of experimental manipulations of catastrophizing on expected pain, experienced pain, and on physical performance during a Knee-Extension-Flexion task (KEF-task), in a sample of outpatients with chronic recurrent low back pain where catastrophizing scores are expected to be higher. An attempt was made to temporarily reduce the level of catastrophizing in participants with chronic recurrent low back pain.

It was hypothesized that participants in the experimental group, where attempts were made to experimentally reduce the level of catastrophizing, would expect less pain, would experience lless pain, and would perform better on the KEF-task as compared to those in the control group, and that these group differences would be associated with a reduction in catastrophizing in the former group. Furthermore, to controll for the possible effects of a nonspecific mood increase due to the catastrophizing reduction, participants in the control group were given a generally positive message, aimed at inducing a general positive mood. 


\section{Methods}

\section{Participiants}

Participants were recruited from patients with chronic recurrent low back pain that were referted for physiotherapy to the department of Rehabilitation Sciences \& Physiotherapy of the Ghent University Hospital and from two physiotherapy practices in Ghent, Belgium. They were asked if they were interested in participating in an experiment in which a new device for use in rehabilitation and the response of people to the use of this device is examined. They were given written information about the experiment and, if interested, completed an informed consent form, the $\mathrm{PCS}$, and a form with socio-demographic questions. After completion of these forms, participants were alternately allocated to the experimental group or the control group. These alternate group allocations were made separately for men and women.

Participants between 18 and 65 years old, who had chronic recurrent low back pain (defined as back pain with a duration of at least three months), and who were currently experiencing an episode of low back pain were included for the study. Participants were excluded if they suffered from a rheumatic or orthopedic disorder in the knee of their dominant leg, suffered from a serious somatic disorder that could interfere with the KEF-task (e.g., a serious heart condition), were pregnant, or were familiar with the KEF-task.

A total of 69 persons were found willing and suitable to participate in the current study. Of these $34(49.3 \%)$ were women (mean age: 38.6 years, $S D=13.0$, range $=42$ years) and 35 $50.7 \%$ ) were men (mean age: 39.2 years, $S D=12.3$, range $=40$ years).

\section{Measures}

\section{Pain intensity}

In this study Numerical Rating Scales (NRS) (Jensen \& Karoly, 1992) were used to measure current pain, maximum expected pain during the KEF-task, and maximum experienced pain during the KEF-task. Participants were asked to indicate with a number on a scale ranging from 0 (no pain) to 100 (worst pain ever) the pain they experienced at that moment, the maximum pain they expected, and the maximum pain they actually experienced during the KEF-task. The upper end of the NRS (100) was anchored for each participant by asking him or her to imagine that particular situation in which they had experienced the worst pain ever. The lower end of the NRS (0) was anchored by asking participants to imagine a situation in which they were pain free (this could also be the current situation).

In addition, the pain intensity subscale of the Dutch language version (MPLDLV) (Lousberg, 1994) of the West Haven Yale Multidimensional Pain lnventory (MPI, formerly the WHYMPI) (Kerns et al., 1985) was used. The MPI-DLV is a measure of key aspects of the chronic pain experience, based on the cognitive-behavioral perspective. The questionnaire consists of three major parts, ench containing several subscales. The 13 subscales of the MPIDLV assess pain intensity, pain-related interference, perceived life control, affective distress, social support, responses of significant others to the patient's pain behaviors, and level of participation in typical daily activities. Participants have to indicate the extent to which every item applies to them on a seven-point scale varying from "0 (never)" to "6 (very often)". The pain intensity subscale, which consists of two items, has a minimum possible score of 0 and a maximum possible score of 12. Psychometric properties of the MPI-DLV are satisfying. The temporal stability $(r=.81)$ and the internal consistency (Cronbach's alpha $=.81)$ of the pain intensity subscale is adequate (Lousberg, 1994). Data on construct validity are also adequate. Futhemore, psychometric properties of the Dutch version are roughly the same as those of the American version. 


\section{Catastrophizing}

The Dutch version (an unpublished, 1996 translation by G. Crombez and J.W.S. Viaeyen) of the Pain Catastrophizing Scale (PCS, Sullivan et al, 1995) was used. This is a 13-item seale in which participants are asked to reflect on past painful experiences and indicate the degree to which they experienced thoughts or feelings during pain which are characterized by catastrophizing on a 5-point scale, ranging from 0 (not at all) to 4 (always). Psychometric properties of the Dutch version of the PCS are adequate: it correlates highly $(r=.73)$ with the catastrophizing subscale of the Dutch Pain Cognition List (PCL, Vlaeyen et al., 1990), has a good temporal stability (Pearsons $r=.92$ ) and a high internal consistency (Cronbach's alpha $=$ .85 , Crombez et al., 1998b).

\section{Fear of movement(re)injury}

As measure of fear of movement/(re)injury, Miller et al. (1991) developed the Tampa Scale for Kinesiophobia (TSK). The Dutch transtation (TSK-DV) of the original TSK (Vlaeyen et al., 1995a) was used in this study. It is a 4-point Likert-type scale with scoring alternatives, ranging from. 1 (strongly disagree) to 4 (strongly agree) and consists of 17 items. The total score is calculated after inversion of the individual scores of items $4,8,12$, and 16 . The internal consistency of the TSK in the current sample was adequate (Cronbach's alpha $=.73$ ), given the fact that the number of items is relatively high (17) in relation to the sample size ( $N$ $=69$ ).

At the second TSK measurement (after the experimental/control instruction) a "moment" time-frame was used i.e., participants were explicitly asked to respond to the questions "as it is now, at this very moment" (TSK-state). The internal consistency of the TSK-state in the current sample was adequate (Cronbach's alphat $=.75$ ).

\section{Mamipulation checks}

The PCS-state was used in this study as a manipulation check for the catastrophizing manipulation in condition 1 . For this purpose we slightly accommodated the items of the standard PCS to match a "moment" time-frame and participants were explicitly asked to reflect on the 13 items "as it is now, at this very moment". The intemal consistency of the PCS-state was adequate (Cronbach's alpha $=.90$ ).

The Positwe And Negative Affect Scales (PANAS) (Watson et al, 1988) was used as a check for the negative mood induction in condition 2 and to warrant the credibility of the alleged research question as it was put to the participants (i.e., to examine a new device for use in rehabilitation and the response of people to the use of this device). It consists of 20 descriptors of positive and negative feelings and emotions that had to be rated by participants on a 5 -point scale, ranging from 1 (very slightly or not at all) to 5 (extremely). The "moment" time instruction was used ("you feel this way right now, that is, at the current moment").

\section{Credibility of the manipulation instraction}

The credibility of the manipulation instruction in the experimental and control condition was measured with a NRS. After the KEF-task, just before the debriefing participants had to indicate with a number ranging from 0 (not at all) to 100 (absolutely) how credible the instruction as given to them by the experimenter was.

\section{Dummy questionnaire}

A dummy questionnaire was developed especially for this study, consisting of 15 fake questions purported to measure "psychological stability" and "emotional competence". Participants had to indicate to what extent every item applied to them on a 5-point scale, ranging from 1 (not at all) to 5 (very much). This questionnaire was referred to in condition 2 , 
where participants were informed that close inspection of this questionmaire has revealed that they were psychologically stable and emotionally intelligent and competent people. The purpose of this instruction in condition 2 was to control for the possible non-specific effects of the pain-catastrophixing induction in condition 1 . This was done by the attempt to induce a general positive state of mind.

\section{Behavioral test}

\section{Apparatus}

The KEF-task was performed on a Biodex system 3 (Biodex Medical System, Inc., New York) at a velocity of $180^{\circ} / \mathrm{sec}$. This test is minimally back straining so that any differences in performance between participants can not be attributed to differences in back pathology or pain intensity. The endurance protocol of the KEF-task was run. One series was done with the dominant $\mathrm{leg}$, consisting of a maximum of 20 consecutive extensions and flexions of the knee. The KEF-task provides information on the peak strength of the muscles relative to the body weight ( $P$ eak torque/BW), the variability of the muscle strength within a series (Coefficient of variance), and the range of motion (ROM) ${ }^{13}$. A lower peak force, a higher variability, and a smaller ROM are indicative of avoidance and suboptimal effort on the test. All participants were unfamiliar with the KEF-task.

\section{Procedure}

On arrival, participants were all informed that the study was investigating a new device for measuring physical fitness and functional muscle capacity in rehabilitation programs and how people respond to this. They were told that for this purpose they would be asked to complete some questionnaires during the course of the experiment and to participate in a behavioral task, using this dewice. Participants then, after instruction, completed the NRS current pain and expected pain, the first part of the MPI-DLV, the PCS-state, the TSK, the PANAS scales, and the dummy questionnaire. In order to permit some time between this and the second measurement, which was just before the KEF-task, participants were given a half an hour break, during which they were allowed to watch a documentary. Following the break, the KEF-task was introduced. The participants were asked to take a seat on the Biodex unit and all the straps were fastened and the participants were positioned appropriately. They were allowed a practice trial in which the experimenter first passively stretched and bent the dominant leg to the maximum deflection points. Then, participants were asked to actively do the same twice. Consequently they were told that the experiment consisted of one series of knee extensions and flexions with their dominant leg. Instructions in both conditions were identical, except for some sentences amed at (temporarily) reducing catastrophizing in condition 1, and inducing a general positive state of mind in condition 2 . The instructions in condition 1 were partly based on the rationale that is used in graded exposure therapy for pain patients with high levels of fear of movement/(re)injury (Vlaeyen et al., 2001). Thus all participants were tolld that:

"In a moment I will ask you to flex and extend the knee of your leftright leg as quickly and as forcefully as possible, until you can"t continue any further." For participants in condition I, the instruction continued:

"Before we continue however, we are pleased to inform you that close inspection of your medical fite has revealed that you fortunately belong to the large group of people with chronic benign low back pain. This is confirmed once more by the results of the questionnaires you completed at your arrival that have revealed that your way of answering to these questions exactly matches that of a typical patient with chronic benign low back pain. Extensive

\footnotetext{
13 The ROM is expressed in degres and is indicative of the range of the movement from extension to flexion.
} 
research has demonstrated that in case of chronic benign low back pain there is no direot relationship between the presence of low back pain and injury to the back. So in your tase this means that pain or increase in pain during movement or other activity, just as in other people with this condition, although unpleasant and disagreeable, is absolutely harmless, and that nothing serious can happen such as damage to the back or pemanent injury. Although very unlikely, any discomfortpain or increase in pain during the exercise you are about to do is only temporary and will dissolve without negative consequences within several minutes after completing the test". For participants in condition 2 , the instruction continued:

"Before we contimue however, we are happy to inform you that close inspection of the questionnaires that you completed at your arrival has revealed that your way of answering to these questions exactly matches that of psychologically stable, and emotionally intelligent and competent people".

Subsequently, participants were asked to complete the NRS for eurrent pain and expected pain, the PCS-state, the TSK (with "moment" time instruction), and the PANAS. They then started with the KEF-task, after being instructed to flex and extend their knee as quickly and as forcefully as possible, until they could not continue any further. A series was halted after 20 extensions and flexions of the knee. During the test they were verbally encouraged by the experimenter ("go on", "come on"). Immediately after the KEF-task participants were asked to complete the NRS for maximum experienced pain during the KEF-task and for current pain, the PANAS, and the NRS for credibility.

Finally, all participants were thoroughly debriefed. During the debriefing process participants were informed about the actual research question and about the reason why the experiment was performed in this way. With respect to the dummy questionnaire, participants were informed that this is a fake questionmaire that does not bear any reference to psychological stability and emotional competence. Finally, they were given the opportunity to ask any questions they might still have.

\section{Statistical analysis}

The General Linear Model (GLM) with repeated measures procedure was used to examine whether the catastrophizing reduction and the positive mood induction were successful. We expected a larger decrease in the level of catastrophizing in the experimental group than in the control group. Also, we expected the level of positive mood to increase in both the experimental and the control group.

The GLM with repeated measures was used to examine whether participants in the experimental group expected less pain than those in the control group.

The univariate GLM was used to examine whether participants in the experimental group had a lower level of maximum experienced pain during the KEF-task than participants in the control group. This analysis was adjusted for the level of pain just prior to the experimental/control instruction.

Independent-samples $t$-tests were performed to examine whether participants in the experimental group had a higher peak torque/body weight, a lower coefficient of variance (both extension and flexion), and a higher range of motion than participants in the control group.

\section{Results}

\section{Descriptive results}

In Table 1 , descriptives are shown for the variables age, duration of the back pain, pain catastrophizing ( $\mathrm{PCS}$ ) prior to the experiment, fear of movement prior to the experimental/control instruction (TSK), pre-experimental pain intensity (MPI), positive affect 
and negative affect prior to the experimental/control instruction, and credibility of the experimental/control instruction (ctedibility).

On average, participants in the experimental group were older than participants in the control group $(n(65)=2.56, p<.05$, wo-tailed). There were no differences between the experimental group and the control group with respect to the duration of the back pain (t(64) $=1.05, \mathrm{n} . \mathrm{s}$, two-tailed), pain catastrophizing prior to the experiment $(t(54)=-0.20$, n.s., twotailed), fear of movement(re)injury prior to instruction $(t(67)=-1.09$, n.s., two-tailed), preexperimentall pain intensity $(t(67)=0.43$, n.s., two-tailed), positive affect $(t(67)=0.60$. n.s., two-tailed), negative affect $(\mathrm{t}(67)=-1.08$, n.s., two-tailed, and credibility of the experimental/control instruction $(t(67)=-1.62$, n.s., two-tailed). With respect to credibility however, there was one participant in the experimental group who reported a credibility score of only 20 . Post hoc analyses in which this score was treated as an outlier did not change the results.

\section{Manipulation check}

The GLM with repeated measures procedure was used to examine whether the catastrophizing reduction was successful. "Group" (the experimental group versus the control group) was entered as between-subjects factor and "time" (PCS-state before experimental/control instruction, i.e., PCS-state time 1 and PCS-state after experimental/control instruction, i.e., PCS-state time 2,) as within-subjects factor. For the sample as a whole there was no significant "time $\times$ group" interaction effect $(F(1,67)=0.26, n . s$.). This means that the catastrophizing reduction in the experimental group was not successful.

However, it should be noted that contrary to what we expected in an outpatient sample, PCS scores were quite $\mathbb{l o w}$, both generally (PCS scores: Mean $=15.20, S D=9.95$ ) and just prior to the experimental/control instruction (PCS-state time 1): Mean $=14.80, S D=9.55$ ). In fact, the levels of catastrophizing were comparable to those found in healthy and non-patient samples (see e.g., Severeijns et al., in press; Sullivan et al., 1995; Van Damme et al., 2000 ). These low levels of catastrophizing may leave little room for attempts to experimentally reduce them and may indicate the presence of a floor effect. Furthermore, associations between catastrophizing and various pain outcomes especially hold for those who catastrophize, i.e., those who have higher levels of catastrophizing about pain.

For this reason we decided to limit all subsequent analyses to those participants with the highest levels of catastrophizing prior to the experimental/control instruction (PCS-state time 1). More specifically, we analyzed the data of the participants whth catastrophizing scores (PCS-state time 1) falling in the highest tertile. The catastrophizing scores of this subgroup $(M$ wan $=25.57, S D=5.74)$ appeared to be comparable to the levels of catastrophizing found in other samples of patients whth chronic pain (see e.g., Crombez et al, 2000; Geert Crombez et al. 1999; Osman et al., 2000; Van Damme et al., 2002a). All the resuls reported below are limited to the paticipants with highest tertile PCS-state time I scores.

It Table 2, the descriptives of Table 1 are replicated for this highest tertile subgroup. The differences between the means of the experimental and control group on the variables age, PCS, TSK and positive alfect were considered relevant ${ }^{14}$.

\footnotetext{
When wsing independent $t$-tests, a significant difference between the experimental and controt group was only found for TSK. However, due to a power problem (small n) also non-significant differences might be retevani. We defined a relevan difference as a differenee that was greater than half the pooled standard devation of the experimental and the control group for a particular variable.
} 

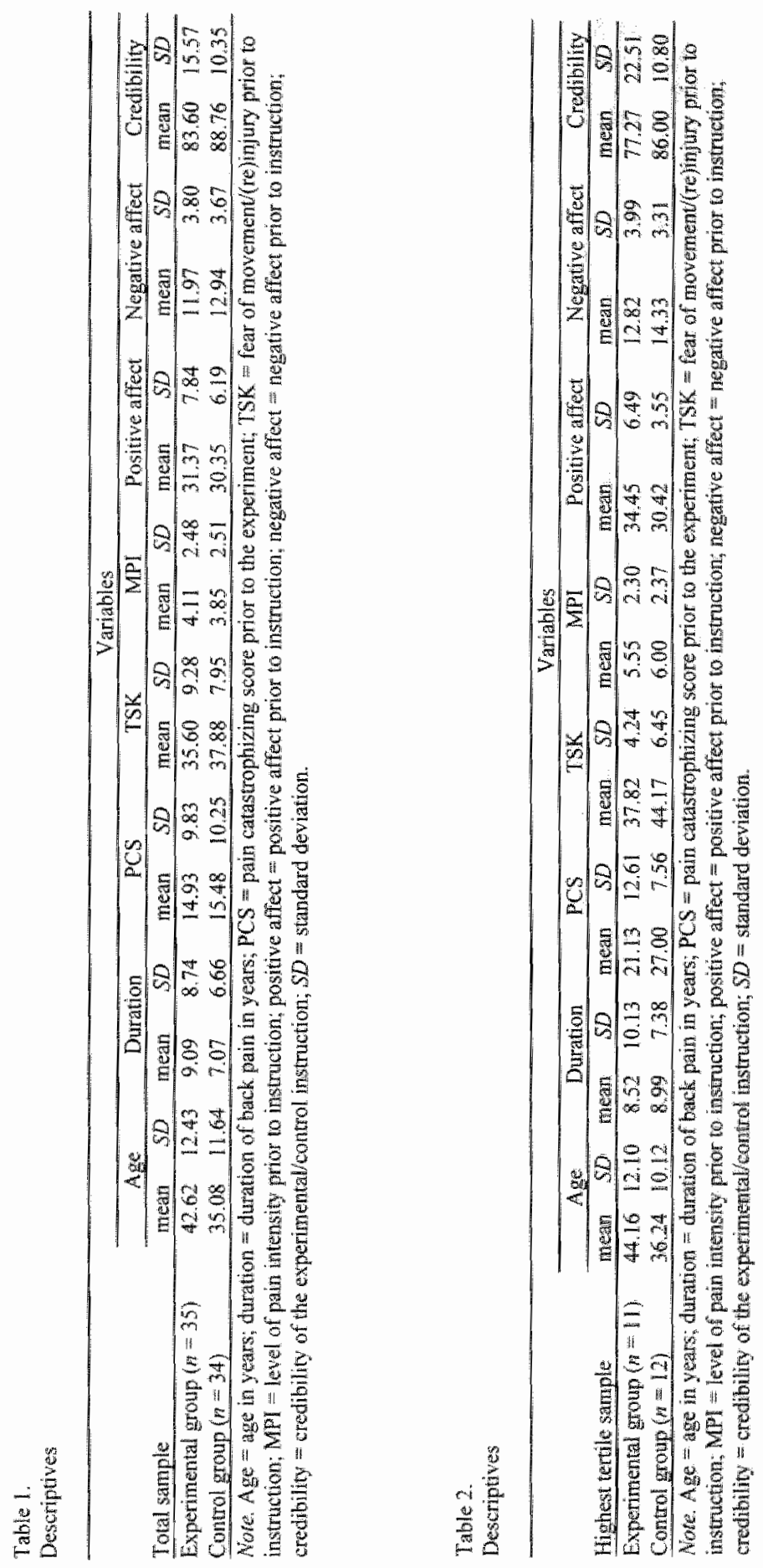
In this subgroup we did find a significant "time $\times$ group" interaction effect $(F(1,21)=$ $4.34, p<.05)$, indicating that the catastrophizing reduction in the experimental group was successful. Table 3 shows the descriptives for the catastrophizing manipulation for the sample as a whole and for the highest tertile subgroup.

Table 3.

Descriptives for the catastrophizing reduction

\begin{tabular}{|c|c|c|c|c|c|c|c|}
\hline \multirow[b]{3}{*}{ Group } & & \multicolumn{6}{|c|}{ Sample } \\
\hline & \multirow[b]{2}{*}{ Time } & \multicolumn{3}{|c|}{ Total sanple } & \multicolumn{3}{|c|}{$\begin{array}{l}\text { Highest tartile } \\
\text { subgroup }\end{array}$} \\
\hline & & Mean & SO & $n$ & Mean & $S D$ & $n$ \\
\hline \multirow[t]{2}{*}{ Experimential } & 1 & 15.09 & 8.48 & 35 & 24.82 & 4.75 & 11 \\
\hline & 2 & 14.20 & 8.67 & & 19.82 & 6.05 & \\
\hline \multirow[t]{2}{*}{ Control } & 1 & 14.44 & 10.65 & 34 & 26.25 & 6.66 & 12 \\
\hline & 2 & 14.32 & 10.77 & & 25.42 & 7.18 & \\
\hline
\end{tabular}

Nole. $S D=$ standard deviation. Catastrophizing scores at time 1 were measured prior to the experimental/control instruction (PCS-state time 1), catastrophizing scores at time 2 were measured following the exwerimental/control instruction (PCS-sitate time 2 )

Similarly, the GLM with repeated measures procedure was used to examine whether the positive mood induction was successful. "Group" (the experimental versus the control group) was entered as between-subjects factor and "time"(the PANAS positive affect scores before and after the experimental/control instruction) as within-subjects factor. Contrary to what we expected there was no significant effect of "time" $(F(1,21)=1.76$, n.s.). This indicates that the positive mood induction in the control group failed and that the reduction of catastrophizing in the experimental group was not accompanied by a positive mood increase either.

Hypothetically though, the attempt to induce a positive mood in the control group as well as the catastrophizing reduction in the experimental group might also result in a lower level of negative mood. The results of the GLM with repeated measures procedure approached significance, i.e., there was a nearly significant effect of "time" $(F(1,21)=3.91, p=.06)$, indicating that for the sample as a whole there was an overall (i.e., in both the experimental and the control group) tendency for a reduction in negative mood. Table 4 shows the effects of the positive mood induction on positive mood as well as its effects on negative mood.

Table 4.

Effechs of the positive mood induction on positive and negative nood

\begin{tabular}{|c|c|c|c|c|c|}
\hline \multirow[b]{3}{*}{ Grous } & \multirow[b]{3}{*}{ tima } & \multicolumn{4}{|c|}{ Variables } \\
\hline & & \multicolumn{2}{|c|}{ Posiltive mood } & \multicolumn{2}{|c|}{ Negatiwe nood } \\
\hline & & Mean & $S D$ & Mean & $S D$ \\
\hline \multirow[t]{2}{*}{ Experimental $(n=\approx \|=$} & 1 & 34.45 & 6.49 & 12.82 & 3.99 \\
\hline & 2 & 32.91 & 8.43 & 11.27 & 1.49 \\
\hline \multirow[t]{2}{*}{ Control $(n=12)$} & 1 & 30.42 & 3.55 & 14.33 & 3.31 \\
\hline & 2 & 29.67 & 526 & 13.08 & 4.72 \\
\hline
\end{tabular}

Nore. Only the dath for participants with the hightest tertile Calastrophizing scores (time 1) are represented in the table. SD = standond deviation. Positive and negative mood scores at time 1 were measufed prior to the experimental/control instruction, positive and negative mood scores at time 2 were measured following the axperimentoldont al instruction. 


\section{Erfects of reducing catastrophizing}

For the dependent variables peak torque/BW and coefficient of variance there were origimally two measures, one for extension and one for flexion. The variables peak torque/BW extension and -flexion correlated highly $(r=90, p<.01)$. For this reason we decided to combine both measures into one variable: mean peak torque/BW ((peak torque extension + peak torque flexion)/2). Descriptives for the dependent variables (expected pain, fear of movement/(re)injury, maximum experienced pain during the $\mathrm{KEF}$ task, mean peak torque/BW, coefficient of variance extension and -flexion, and ROM) are shown in Table 5 .

Trable 5 .

Descriptives for the dependent variables.

\begin{tabular}{|c|c|c|c|c|c|c|}
\hline \multirow[b]{2}{*}{ Variables } & \multicolumn{3}{|c|}{$\begin{array}{l}\text { Experimental group } \\
m=11\end{array}$} & \multicolumn{3}{|c|}{$\begin{array}{c}\text { Control group } \\
y=12\end{array}$} \\
\hline & Mean & $S D$ & Min-max & Moan & $S D$ & Min-max \\
\hline Fear of movement (TSK) time 1 & 37.82 & 4.24 & $30-44$ & 44.17 & 6.45 & 34.56 \\
\hline time 2 & 38.82 & 4.89 & $32-46$ & 43.67 & 5.58 & $35-51$ \\
\hline Expected pain time $\mathbb{1}$ & 40.91 & 20.47 & $5-75$ & 45.42 & 22.31 & $10-75$ \\
\hline time 2 & 39.09 & 21.19 & 0.75 & 46.67 & 24.53 & $10+75$ \\
\hline Maximum experienced pain & 30.45 & 18.09 & $0-60$ & 38.33 & 30.02 & $5-90$ \\
\hline Mean peak torque/BW & 62.91 & 42.43 & $24.00-159,00$ & 61.38 & 45.90 & $9.55-143.85$ \\
\hline Coefficient of variance extension & 1287 & 4.11 & $5.80-17.10$ & 17.66 & 5.33 & $9.40-28.90$ \\
\hline flexion & 16.44 & 6.41 & $9.00 \cdot 27.10$ & 18.87 & 12.61 & $0.00 \mathrm{w} \quad 49.30$ \\
\hline Range of movement (ROM) & 92.35 & 9.86 & $76.60-107.20$ & 84.64 & 10.25 & $62.90-101.00$ \\
\hline
\end{tabular}

Nore. Only the data for participants with the highest tertile catastrophizing scores are represented in the table. $S D=$ standard deviation, Min-max minimum and maximum scote the the sample.

The GLM with repeated measures procedure was used to test the hypothesis that participants in the experimental group would show a greater reduction in expected pain than those in the control group. There was however no significant "time (before and after instruction) $\times$ group (experimental versus control group)" interaction for expected pain $(F(1$, 21) $=0.80$, n.s.). This means that, contrary to our hypothesis, there was no difference in the reduction of expected pain between the experimental and the control group.

The uniwariate GLM was used to examine whether participants in the experimental group, as compared to those in the control group, would report a lower level of maxmum experienced pain during the KEF-task, controlling for the level of current pain (as measured with the NRS current pain prior to the experimental/control instruction). The results demonstrated that, contrary to what we expected, this was not the case $(F(1,20)=0.15, n .5$.$) .$

Independent samples r-tests were performed to test the hypotheses that participants in the experimental group would have a higher peak torque (mean peak torque/BW), a lower coefficient of variance (extension and flexion), and a higher ROM, as compared to those in the control group. Contrary to our hypotheses, no significant effects were found for peak torque $(r(21)=0.08$, n.s., one-tailed $)$, nor for coefficient of variance flexion $(t(21)=-0.57$, n.s., one-tailed). However, we found a significant effect for the coefficient of variance extension $(r(21)=-2.39, p<.05$, one-tailed) and for the $\operatorname{ROM}(2(21)=1.83, p<.05$, onetailed). This indicates that participants in the experimental group performed better on the KEF-task with respect to these two measures than participants in the control group.

However, it can not simply be concluded that the reduction in the level of catastrophizing contributed to these significant effects because we did not perform any pre-measurements on the dependent variables. In order to more substantiate such a conclusion we would expect the 
reduction of catastrophizing to be correlated with the variables $R O M$ and coefficient of wariance extension. It should be noted though that due to small $n$, these correlations are difficult to interpret. Nevertheless, we calculated Kendall's tau (because of the small w)

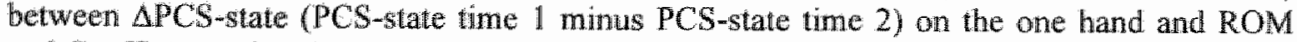
and Coefficient of variance extension on the other hand. Separate conrelations were calculated for the experimental and the control group. As Table 6 demonsitrates, none of the correlations were significant.

Tablew 6.

A ssociation (Kendall"s tati) between the change in the level of calastrophizing and dependerit variables

\begin{tabular}{|c|c|c|}
\hline \multirow[b]{2}{*}{ Group } & \multicolumn{2}{|c|}{ Dependent variables. } \\
\hline & Range of movement & $\begin{array}{c}\text { Coentucient of variance } \\
\text { extension }\end{array}$ \\
\hline Experimental group & $-.28(10.5)$ & 31 (ns.s) \\
\hline Control group &. $.06(n . s)$ & $-.09(\mathrm{n} . \mathrm{s})$ \\
\hline
\end{tabular}

Finally, we post hoc examined whether each of the four descriptive variables on which there were relevant differences between the experimental and control group (Table 2: age, PCS, TSK, and positive affect) affected the significant differences between experimental and control group on ROM and coefficient of variance extension. For this reason we performed two series (one series with ROM and one series with coefficient of variance extension as dependent variable) of univariate GLM analyses. In these analyses "condition" was entered as between subjects factor and the four variables on which there were relevant differences between the experimental and control group (age, PCS, TSK, and positive affect), were entered separately as covariates. Participants in the experimental group were older, catastrophized less, were less afraid of movement/(re)injury, and had a more positive mood as compared to those in the control group. The results demonstrated differences with respect to both dependent variables. Every covariate separately rendered the effect of "condition" on ROM non-significant. With respect to coefficient of variance extension, only PCS (the preexperimental level of catastrophizing) rendered the effect of "condition" non-significant. Again, it should be noted that due to small $n$, these results are difficult to interpret Furthermore, although apparently these variables did contribute to the significant differences between the experimental and control group on the variables ROM and coefficient of variance extension, their contribution was not substantial given the fact that the estimated marginal means (i.e., the group means on the dependent variables adjusted for the effects of the covariate) only marginally differed from the group means on the dependent variables.

\section{Discussion}

At first glance, the attempt to reduce the level of catastrophizing about pain did not appear to be successful. However, as can be seen in Table 1 and Table 3, the tevel of catastrophizing was unexpectedly low (both prior to the experiment as measured with the PCS and prior to the experimental/control instruction as measured with the PCS-state time 1), considering the fact that the sample was comprised of outpatients who suffered from chronic recurrent low back pain. In fact, catastrophizing scores in the current sample were comparable to those found in community samples (Osman et al., 2000; Severeijns et al., 2004) and in pain-free samples (Severeijns et al., in press; Van Damme et al., 2000). These low levels of catastrophizing may 
signify that there was a floor effect that prevented the PCS-state time 1 scores from being reduced to the extent that the reduction could have bad a significant effect on the dependent variables. Furthemore, associations betwoen catastrophizing and various pain outoons variables are know to bold for those who catastrophize about pain, that is, those people who thave farly high levels of catastrophizing. For this reason we chose lo thit the analyses to those participants with PCS-state time 1 scotes (i.e., prior to the experimentabountrol instruction) falling into the highest tertile. These highest tertile PCS-state time 1 scores appeared to be more comparable to the PCS scores usually found in other samples of patients with chronic pain. Indeed, when limiting the analyses to this sub sample, our atcmpt to reduce the level of catastrophizing in the experimental group was suceessful. This strecessful reduction of catastrophizing in the highest tertile subgroup is also an indication that there was indeed a floor effect in the sample as a whole where catastrophizing levels were gute low. Perhaps this floor effect could have been prevented with a somewhat moderated procedure. On arrival participants were informed only in general terms about a behavioral task that they would be asked to participate in (see procedure section). After this general information they were asked to complete, amongst others, the PCS-state time 1. Apparently, this general information about a "behavioral task" was not appraised as very threatening by the participants judging by the low PCS-state time I scores following this infomation. Consequentty, there was not much room for reducing these already low PCS state scores. Giving more detailed information about the $\mathrm{KEF}$-task, perhaps even stressing threat-inducing (allhough fictitious) aspects of the task, would possibly have increased the resulting PCS state scores, thereby leaving more room for a reduction following the experimental instruction.

Contrary to what we expected we neither succeded in inducing a positive mood directly (via the control instruction in the control group), nor indirectly (in the experimental group via experimentally reducing the level of catastrophizing) although there was an overall marginally significant general and beneficial effect on negative mood. It seems plausible that the reduction in catastrophizing about pain was accompanied by a reduction in negative mood. This would be consistent with a previous prospective study by Keefe et al. (1989) in which it was demonstrated that catastrophizing measured at time 1 predicted depression at time 2, even when controlling for depression measured at time 1 .

Although participants in the experimental group performed better on some KEF-task parameters as compared to those in the control group, that is, they had a higher range of motion and a lower coefficient of variance extension, we did not find conclusive evidence that this better perfonnance can be ascribed to the reduction in catastrophizing. In this respect it would have been helpful to have a baseline measurement or premeasurement ou the dependent variables. We then could have examined whether the reduction in catastrophizing was associated with a reduction on these dependent variables. However, the decision not to have a baseline measurement was made deliberately because of the possibility of practice effects that might influence the effects of our instructions. Indeed, there are indications that performing a certain movement task twice leads to a change in the prediction of the painfulness of that task (Crombez, Eccleston, Vlaeyen, Vansteenwegen, Lysens \& Felen, 2002; Goubert et al., 2002). Post hoc, some covariables (age, pre-experimental level of caltastrophizing, fear of movement(re)injury prior to instruction, and positive affect prior to instruction) appeared to contribute to the differences in $\mathrm{KEF}$-task performance befween the experimental and control group. However, due to small $n$, these results are difficult to interpret. Furthermore, the actual contribution of the covariates was rather marginal.

These results may indicate that there is a variable not controlled for in the analyses that moderates the relationship between catastrophizing and the dependent variables in the current study. Neuroticism may be that factor. For example, Goubert et al. (2004) found that neuroticism moderated the relationship between pain severity and catastrophizing. 
Alternatively, the significant differences between the experimental and control group on the two $\mathrm{KEF}^{-}$-task parameters may not be related to the reduction in catastrophizing at all. Finally, there is also the possibility that, as the data seem to suggest, the reduction in catastrophizing in the experimental group is related to the better performance on the two KEF-task parameters. Future studies on the causal status of catastrophizing should include baseline measures of the dependent variables as well as a measure of neuroticism.

Besides the fact that the significant differences between the experimental and control group on ROM and coefficient of variance extension can not conclusively be ascribed to the reduction in catastrophizing in the former group, no significant differences between the two groups were found on the other dependent variables: pain, neither expected pain nor experienced pain, peak torque, and coefficient of variance flexion. It should be noted though that even in the subgroup of participants with highest tertile catastrophizing scores, pain levels of the sample as a whole prior to the experiment were only low to moderate (mean MPI-DLV pain and mean NRS current pain were 5.78 and 35.00 respectively, with maximum possible scores of 12 and 100 respectively). Furthermore, the KEF task is not a back straining task (although participants did not know this) and may not have been perceived as such either by the participants, leaving the levels of expected and maximum experienced pain relatively insensitive to the effects of experimental manipulations of catastrophizing. This problem could perhaps have been overcome by using a somewhat different procedure. For example, we could have stressed the pain inducing properties of the KEF-task (although this is not true) during the introduction phase of the experiment. This may have left the thus elevated expected pain level in the experimental group more sensitive to change as a result of a reduced level of eatastrophizing. Furthermore, a task known or expected to generate some painful sensations and catastrophizing appraisals in participants with chronic recurrent low back pain, would perhaps have been more appropriate, e.g., a lifting task.

In this experiment we attempted to temporarily reduce the level of catastrophizing. For this reason the instruction of the PCS was adapted with a momentary time frame. That is, just before and after the experimental/control instruction, participants were asked to reflect on the 13 PCS items "as it is right now, at this very moment". However, this state catastrophizing may be different from catastrophizing as measured with the regular PCS which has more traitlike qualities, and the latter may be the more crucial in affecting KEF-task performance but also the more difficult to change with a relative simple instruction. A second measurement with the regular PCS after the experimental instruction would have been informative. Alternatively, the PCS-state may not have been specific enough because in the items of the PCS-state no reference is made to the KEF-task. An especially for this experiment adapted version of the PCS in which explicit reference was made to the KEF-task may have been better.

Finally, there is the methodological problem we already touched upon, that should be mentioned here. Limiting the analyses to a subgroup of participants with high state catastrophizing scores, reduced the statistical power of particularly the correlational analyses and the analyses with the covariates, due to small numbers $(n=11$ in the experimental group). This makes a meaningful interpretation of the results of these analyses rather difficult, if not impossible. In similar future studies, participants should be selected also on having high catastrophizing scores.

In conclusion, the results of the current study demonstrate that a relatively high level of state catastrophizing can be (temporarily) reduced rather easily by means of a simple instruction. These results are in accordance with the results of a previous study with healthy participants in which the level of state catastrophizing was induced successfully (Severeijns et al., in press). Both studies raised some new questions that need to be examined in future 
experiments in order to gain more insight into the possible causal status of pain catastrophizing.

\section{Acknowledgments}

Thanks are expressed to Prof. Dr. A. Arniz and E. Schouten of the Department of Medical, Clinical, and Experimental Psychology of the Maastricht University for their valuable methodological and statistical suggestions. 


\title{
CHAPTER 10
}

\author{
General discussion
}

"He that is umeasy at every lithle pain is never without some ache" Proverb 


\section{General discussion}

The objective of this thesis was to examine the role of pain catastrophizing in (chronic) pan. For this purpose six studies were performed, each addressing some specific research questions relating to this main objective. The aim of this final chapter is to present a summary and discussion of the main findings and of the strengths and limitations of the studies. Finally, some tentative conclusions and recommendations are given.

\section{Main findings}

In response to the research questions as formulated in the outline of this thesis, the main findings of the six studies are summarized point by point below.

1. Pain catastrophizing predicts pain intensity, pain-related disability, and psychological distress, independent of the level of plysical impaiment and across different pain subgroups (Chapter 4)

2. Physical impaiment makes a unique, though modest contribution to the prediction of pain intensity, pain-related disability, and psychological distress (Chapter 4)

3. Pain catastrophizing and physical impaiment are not related (Chapter 4)

4. Pain catastrophizing makes a significant contribution to the prediction of several aspects of the general health status of people, notably mental health, general health perception, social functioning, and vitality, across different pain subgroups in the general commumity (Chapter 5)

5. A three-factor structure of the Pain Catastrophizing Scale (PCS) is confirmed in a large community sample, although a one-factor structure is also found justifiable. The factor structure of the PCS is invariant across different pain subgroups (Chapter 5)

6. The reliability of the PCS in the general community is adequate and comparable to the reliability of the PCS in other populations. There are indications for concurrent validity of the PCS in the general community (Chapter 5)

7. Pain catastrophizing, pain intensity, and the number of pain locations are independently from each other related to specialist consultation, use of pain medication, and absenteeism or work disability. These relationships hold across different subgroups of people with a current episode of pain in the general community. The relationships in question could not be confirmed prospectively, nor is the relationship between pain catastrophizing and these health indices moderated by pain intensity and the number of pain complaints (Chapter 6 and Chapter 7 )

8. The number of pain complaints and pain catastrophizing, rather then pain intensity per se, independently predict the development of elronic pain complaints in people with musculoskeletal pain in the general community (Chapter 7)

9. The level of pain catastrophizing in relation to a specific situation (i.c., the state-like form of catastrophizing) can be manipulated rather easily with a relatively simple instruction (Chapter 8 and Chapter 9)

10. Temporarily increasing the level of pain catastrophizing not necessarily leads to a higher level of expected and experienced pain or escape/avoidance behavior in experimentally induced pain in a sample of healthy subjects (Chapter 8)

11. Temporarily reducing the level of pain catastrophizing not necessarily results in a reduction in expected pain, a lower level of experienced pain, and task performance during a Knee-Extension-Flexion task on a Biodex system 3 (Chapter 9). 


\section{Discussion}

Physical impairment, pain, and pain catastrophizing

The results of the frist study indicate that pain catastrophizing is not simply an epuphenomenon of the severity of the pain problem as expressed in the level of physical impaiment. Moreover, it does not even appear to be related to physical impairment (as measured with MEDICS, Rudy et al., 1990) at all. With respect to pain, a similar picture emerges. Although related to pain, pain catastrophizing independenty has unique explanatory power in relation to several pain-related outcome variables examined in this thesis, such as general health status, specialist consultation, use of medication, and absenteeism. Also, the relationship between pain catastrophizing and several outcome variables is not moderated by pain. These results are a further confirmation that, although related to pain, (and, as discussed in Chapter 3 , to basic emotional constructs such as neuroticism, depression, and anxiety), pain catastrophizing is a separate and valid construct that has its own merits in the study of pain.

The data show that, notwithstanding the fact that pain catastrophizing also occurs in the absence of pain, it particularly manifests itself in the presence of pain. Furthermore, there appears to be an important difference between clinical populations and the general population with respect to the level of catastrophizing. The mean score on the Pain Catastrophizing Scale (PCS) in the clinical sample used in the study that is described in Chapter 4 was 26.31. (this mean is not reported in the study but was calculated post hoc), while the mean PCS score in the community sample used in the population studies described in Chapter 5 and 6 was 10.00 (sample as a whole). These differences between clinical and population samples were found in other studies as well (e.g., Osman et al., 2000 ) and can not be explained by the chronicity of the pain complaints in clinical samples because $81.2 \%$ of the population sample used in the studies described in Chapter 5 and 6 reported chronic complaints as well. Furthermore, there were no differences in the level of catastrophizing between participants with acute or sub acute pain and participants with chronic pain complaints.

These findings may give us some clues about the nature of catastrophizing. The fact that pain catastrophizing also occurs in the absence of pain implies that it has some trait-like qualities, while the fact that it is more pronounced in the presence versus the absence of pain and in clinical versus population samples suggests that it also has some situation-specific qualities. This is in line with the proposed model of pain catastroplizing that was presented in Chapter 3 (Figure 1) and is based on the transactional stress and coping model of Lazarus and Folkman (1984). In this proposed model, pain catastrophizing is conceptualized as a set of appraisals and negative belies, the latter with both dispositional and situation-specific qualities. In Chapter 3, we already argued in favor of this model as opposed to the Communal Coping Model of pain catastrophizing (CCM) in which catastrophizing is viewed as boing part of a broader, interpersonal or communal coping style in which it serves a social communicative function. The main objection to the CCM is that in its current formulation it delines catastrophizing, which is explicitly cognitive in nature, in terms of one of its potential functions or effects. Furthermore, we argue that the predictions made by the CCM can also be accounted for by the appraisal model of catastrophizing. Finally, we contend that the fact that it has interpersonal correlates is intrinsic to the very nature of catastrophizing.

\section{Pawn catostrophizing and setting}

The fact that the level of pain catastrophizing varies with the absence or presence of pain and with pain intensity may seem quite logical. What about the fact that people in clinical settings catastrophize more about pain than people in the general population? What is it about clinical settings that causes people to catastrophize considerably more about pain than in the general population? The current thesis does not permit an empirically based answer to this question, 
although it appears that it does not have anything to do with physical impairment or with chronicity.

A possible explanation might be that a clinical setting entails a greater risk of conveying some subtle threatening messages to people with (chronic) pain about the possible negative consequences of the pain problem, thereby fostering catastrophic ideation about pain (e.g., seeing disabled and/or handicapped patients, certain remarks and advice of healthcare providers) in people who are already at risk, i.e., who have a disposition to catastrophize about pain. Indeed, there are indications that certain aspects of the doctor-patient relationship (e.g., the way medical or illness information is communicated) are of influence on the level of patient anxiety and depression (Rutter, Iconomou \& Quine, 1996; Shapiro, Boggs, Melamed \& Polle, 1992) which as already discussed in Chapter 3, are related to pain catastrophizing. Other aspects of the doctor-patient relation might be important as well. For example, van Dulmen, Fennis, Mokkink, van der Velden, and Bleijenberg (1995) found that catastrophizing cognitions of patients with functional abdominal complaints who saw the same doctor throughout the consultations reduced more than those of patients who did not see the same doctor all the time. This seems to suggest that in order to counteract pain catastrophizing and its negative consequences, it is important to not only concentrate on patients and how they think about their pain, but to look at several aspects of the setting or context as well.

Alternatively, it is also conceivable that particularly those individuals who catastrophize about their pain complaints are more often referred to a clinical or outpatient treatment center, simply because they more frequently consult their general practitioner for their complaints as compared to those individuals who do not catastrophize about their pain. Indeed, the results of the study described in Chapter 6 suggest that those who catastrophize about their pain have a higher incidence of visiting a specialist for their pain complaints. Clearly, more research is needed in this respect.

\section{Pain catastrophizing and pain subtype}

In the outline of this thesis (Chapter 3 ), the idea was presented that different pain subtypes might differ with respect to their respective perceived threat value and that these differences would cause the specific pain subtype to moderate the relationship beiween pain catastrophizing and the outcome variables examined in this thesis. Although numerous studies have found significant relationships between pain catastrophizing and pain-related outconte variables across different pain subgroups, none of these studies have directly compared different pain subgroups with each other or examined interaction effects between catastrophizing and pain location. The studies presented in Chapter 4 and 6 did so but failed to find any differences between pain subtypes in this respect. Even if different pain subtypes would differ with respect to their inherent threat value (which was not directly examined), the fact that we did not find any moderation effects of pain subtype makes it unlikely that any relationships between pain catastrophizing and pain-related outcome variables would be influenced by such differences.

\section{Factor structure of the Pain Catastrophizing Scale (PCS)}

Although the confirmatory factor analysis statistically supported a three-factor model of the PCS, a one-factor model was found justifable as well. Because all other studies on the factor structure of the PCS have also found evidence for a three-factor model, with the subscales "helplessness", "magnification", and "rumination" as independent but related factors(Osman et al., 2000; Osman et al., 1997; Van Damme et al., 2002a), one might simply argue that the support for a one-factor model found in the current thesis is an artifact of the data gathering methodology (mail out methodology) or of the low PCS scores in the community samples in the current thesis. On the other hand, the use of mail out methodologies is quite common and 
accepted in epidemiological studies. Moreower, more controlled data gathering methods, such as therapist guided administration of questionnaires are rather difficult, if not impossible, to implement in the general community. Perhaps even more important is the fact that a low level of pain catastrophizing may be the rule rather than the exception in the general population. This implies that when the PCS is used for screening purposes in the general community it is adwisable to only use the PCS total score and not the subscales, no matter how valid their use may be in clínical populations.

\section{Pain catastrophizing and pain outcomes}

By and large the results of the studies presented in this thesis confirm the growing notion emerging from recent pain research that pain catastrophizing is a significant factor in (chronic) pain. The studies show that, independently from other variables such as physical impairment, pain intensity, chronicity, age, sex, and across different pain subtypes, pain catastrophizing is related to several pain outcomes, notably pain intensity, disability, psychological distress, general health status, and other health indices such as specialist consultation and use of pain medication. However, it should be noted that these positive results are all based on cross-sectional data that do not permit to pronounce upon the direction of these relationships. Also, some of the associations were rather modest, for example, the associations between catastrophizitig and the health indices examined in Chapter 6 .

The results of the prospective study presented in Chapter 7 of this thesis, failed to confirm the eross-sectional associations between catastrophizing and the health indices examined in Chapter 6 , and only demonstrated a modest relationship between pain catastrophizing and the development of chronic musculoskeletal pain. And although the results of other prospective studies at least suggest that pain catastrophizing is a precursor of various pain-related outcomes rather than a consequence (e.g., Burton et al., 1995 ; Drossman et al., 2000; Keefe et al., 1989), there are also prospective studies that failed to show significant relationships between pain catastrophizing and pain-related outcomes or showed mixed results (e.g., Groth Marnat \& Fletcher, 2000; Jensen et al., 2002; Jensen et al., 2001; Linton et al., 2000; Pfingsten et al., 1997).

How can these equivocal data be explained? Because the different studies used participants with different pain subtypes (e.g., low back pain, phantom limb pain, gastrointestinal pain) and employed different outcome measures, one might argue that the specific type of pain or outcome measure is relevant in this respect. However, as mentioned previously, positive rolationships between pain catastrophizing and pain-related outcomes have been found across various pain subtypes and across various pain-related outcomes, and no interaction effects between pain catastrophizing and pain subtype were found in the studies presented here. Another possibility is that the type of setting is relevant. Indeed, we already argued that the type of setting (e.g., clinical or community) might affect the level of catastrophizing which in tum affects pain-related outcome variables. In this respect, it is likely that due to the low level of catastrophizing in the general community, the role of catastrophizing in the development of future pain problems is less prominent than in clinical settings. A third possibility is that there is a third variable that is botth related to pain catastrophizing and several pain-related outcome variables, and affects the relationships between the two in time. An eligible variable in this respect is neuroticism or negative affectivity. First, it has been shown to be significantly rellated to both pain catastrophizing (Affleck et al, 1992; Drossman et al., 2000; Sullivan et al., 1995) and to various outcome variables (Bisgard et al., 2001; Radanov \& Sturzenegger, 1996). Furthemore, Martin et al. (1996) found that the relationship between pain catastrophizing and physical and psychosocial disability was only significant when neuroticism was removed from the regression model. Finally, Goubent et al. (2004) very recently showed that neuroticism moderated the relationship between pain catastrophizing and 
pain intensity. For future research therefore, it seems important to more thoroughly axanine the role of neuroticism and the type of setting in the relationship between pain catustrophizing and pain-related outcome variables.

Besides neuroticism, there are other trait-like constructs that may be useful as well in the study of pain catastrophizing and its relation to pain. One of these constructs is anxiery sensitivity. This is considered to be one of the three fundamental fears or trat sensitivities (the other two are iliness/injury sensitivity and negative evaluation sensitivity, Reiss, 1991), and is defined as the fear of anxiety-related bodily sensations, based on beliefs that these sensations have harmful consequences (Asmundson \& Taylor, 1996). The results of a study by Asmundson and Taylor (1996) suggested that anxiety sensitivity promoted fear of pain and indirectly, via its influence on fear of pain, promoted escape/avoidance behaviof in people with chronic pain. Another construct is illness/injury sensitivity which is defined as the fear of personal injury (Reiss, 1991). To date, we know of no research on illness/injury sensitivity in relation to pain. Finally, there is the construct of trait anxiety. Hallberg and Cartsson (1998) found that trait-anxiety was positively associated with pain catastrophizing and was negatively associated with abilities to control and reduce pain in a sample of patients with fibromyalgia.

Lilienfeld (1996) proposed a hierarchical factor model in which these constructs are brought together. In this hierarchical factor model the trait sensitivities are considered as som called "second-order" factors, that although separable, share suflicient variance with ach other to form a higher-order or "third-order" trait anxiety ractor. In this view, anxiety" sensitivity like the other trait sensitivities is qualitatively not distinct from trait anxiety because both have a lot of shared variance. Nevertheless, anxiety sensitivity also contains unique variance which is not correlated with trait anxiety. Trait anxiety in turn might covary with other "third-order" factors (e.g., aggressiveness) to form a "fourth-order" negative affectivity or neuroticism factor. Furthermore, the trait sensitivities are held to be divided into even more specific "first-order" factors. In future research it would be interesting to examine the possible vole of pain catastrophizing as a "first-order" factor in this hicrarchical factor model.

\section{The causal status of catastrophizing}

In the last two studies of this thesis we examined whether there are any indications that catastrophizing plays a causal role in (chronic) pain. The results demonstrated that we were able to manipulate the level of catastrophizing in a specific situation rather easily, although the manipulation that was aimed at reducing the level of catastrophizing only worked with participants who had relatively high levels of catastrophizing in the test situation.

We did not find any evidence that inducing catastrophizing in a sample of healthy participants was causally related to expected and experienced pain, and duration of ice-water immersion during a cold pressor task. In the other experiment with patients with chronic recurrent low back pain, we found that participants in the experimental group, where the level of catastrophizing was reduced and who had high catastrophizing scones, performed slightly better on a Knee-Extension-Flexion task than participants in the control group, where the level of catastrophizing was not reduced. Although the data seemed to suggest that the reduction in catastrophizing resulted in a better performance on some KEF-task parameters, no conclusive evidence was found in this respect. The results then of both experiments do not give supportive evidence for the causal status of catastrophizing in (chronic) pain. Nevertheless, it can not simply be concluded that catastrophizing does not play a causall role in (chronic) pain. 
There are several possible explanations for the feeble fundings in both experiments ${ }^{15}$, of which the most important ones concern the generally low levels of pain catastrophizing, the procedures and specific behavioral tasks used in the experiments, and the possible relevance of a personality factor wot accounted for in the experiments: neuroticism.

Some recommendations can be made for future experimental studies on the causal status of catastrophizing. First, participants should be preferably selected on the basis of high scores on the Pain Catastrophizing Scate (PCS) scores because any significant effects are probably most likely to be found within the higher ranges of catastrophizing. Of course the definition of a high PCS score is rather arbitrary and varies with the population from which the sample is drawn. Nevertheless, some considerations might be useful in this respect. The communitybased studies in the current thesis showed rather small to moderate effects of catastrophizing (Chapter 5 and Chapter 6) or almost no effects (Chapter 7). Indeed, mean. PCS scores of the different subgroups in these studies were rather low and varied from 7.82 to 13.53 . This is in line with mean PCS scores found in another community-based sample (13.87, Osman et al, 2000 ) and in a pain-fice sample (16.59, Van Damme et al., 2000). The study with the clinical sample described in Chapter 4 showed some robust effects of catastrophizing. Not surprisingly, the clinical sample in this study had a substantial mean PCS score (26.31), which is in line with mean catastrophizing scores found in other clinical and outpatient samples (Osman et al , 2000; Van Damme et al., 2002a; Van Damme et al., 2000). Based on these considerations, PCS scores well below 20 may be considered low whereas PCS scores higher than 20 may be considered substantial or high. Second, "ecologically valid" tasks should be used, i.e, tasks that resemble activities in daily life and are capable and likely to activate or generate catastrophic ideation, e.g., a lifting task for participants with low back pain. Also, a pre-measurement on the task should be possible without too much interference of practice effects. Third, measures of neuroticism should be included in future experiments.

\section{Strengths and limitations of the studies}

Although an objective and unequivocal measure of "physical impairment" is probably not available, the study presented in Chapter 4 is the first that examined the possible effects of physical impairment on the association between pain catastrophizing and pain outcome variabies. Before, one could still argue that pain catastrophizing is perhaps an epiphenomenon of physical impairment. This study not only shows that this is not the case, but also demonstrates that pain catastrophizing and physical impairment are not related.

One of the strengths of the population-based studies presented in Chapter 5 through Chapter 7 is that, as far as we know, these studies are one of the first to demonstrate the significant role of pain catastrophizing in a large community sample. Data on the role of catastrophizing in the general community are very scarce, and the great majority of the studies on catustrophizing are based on clinical samples or on samples with pain-free subjects. In clinical samples high levels of both mental and physical pathology would be expected which may affect the associations of interest. The results of the studies with pain-free samples may be difficult to generalize to other populations, such as non-clinical pain-suffering subjects. The fact that in this community sample, where levels of catastrophizing were generally quite low, significant associations were found between catastrophizing and the dependent variables of interest (adjusting for variables such as pain intensity, chronicity, age, and sex), means further support for the important role of catastrophizing in (chronic) pain.

The studies presented in Chapter 8 and in Chapter 9 are, to the best of our knowledge, the first attempts to experimentally manipulate the level of pain catastrophizing and examine the

is Although in the experinent with the KEF-task we did find some signilicant results when we limited the analyses to those participatts with relatively high catastrophizing scores, the small $n$ of the remaining sample reduced the statistical power considerably which anade the results rather diflicult to interpret. 
effects of this manipulation on pain and behavior during a performance task. In this respect, these studies would allow for a more conchsive pronouncenent upon the causal stams of pain catastrophizing. Although the results were rather disappointing and no conclusive statements on the causal status of catastrophizing can be made, the studies defintely give useful leads for further research.

Of course, there are also some limitations of the studies presented in this thesis of which most have been discussed in the discussion sections of the respective chapters. The most important ones will be dealt with below.

Although various studies have provided support for a three-factor structure of the Paim Catastrophizing Scalle (PCS), as well as for its internal consistency and reliablity, less attention has been given to the validity of the PCS, especially the construct validity. So far, the construct of catastrophizing has been operationalized solely by means of questionmaires, one of which is the PCS. And although its psychometric properties appear to be adequate, there is no evidence that this questionnaire, as well as other questionnaires that are used to measure catastrophizing (such as the Coping Strategies Questionnaire, Rosenstiel \& Keefe, 1983) cover the construct completely on even are the best option to operationalize the construct. Other means of measuring pain catastrophizing may be appropriate too or may even be a better option. An eligible instrument in this respect is the Implicit Association Test (IAT, Greenwald, MoGhee \& Schwartz, 1998), which is used for measuring individual differences in implicit cognition. For example, Egloff and schmukle (2002) found that anxiety measured with an adapted IAT procedure predicted criterion variables above questionnaire measures of anxiety and social desirability. The IAT could be adapted to measure individual differences in implicit catastrophizing.

The studies presented in Chapter 4 through Chapter 7 are cross-sectional or prospective (Chapter 7). This means that the significant associations that were found do not allow to pronounce conclusively upon the direction of these associations. Nevertheless, they are in the expected direction and are in accordance with other research findings on pain catastrophizing.

One might argue that the significant associations that were found in the population studies were only low to moderate and that the samples in these studies were very large which largely accounts for the significance of these associations. Although this certainly is an appropriate point of criticism, it should also be noted that, as mentioned previously, the levels of catastrophizing in the population studies are quite low. It is not unlikely that these low catastrophizing levels have suppressed the true associations between pain catastrophizing and the dependent variables examined in these studies. The same holds for the experimental studies where the levels of catastrophizing were generally low as well. In these studies it might have been better to select participants with relatively high levels of pain catastrophizing.

In all studies self-report questionnaires were used which are subject to several kinds of bias. Only in the experimental studies we used behavioral measures as well. Furthermore, in the population-based studies a mail-out methodology was used. This means that some kind of selection bias can not be excluded.

\section{Conclusions and recommendations}

The results of the studies presented in this thesis allow for some tentative conclusions and recommendations. First, pain catastrophizing is associated with various negative consequences of pain, such as pain-related disability, pain severity, psychological disuress, a lower quality of life, medical consumption, and the development of chronic pain complaints. These associations hold across different types of pain and different settings, although the associations appear to be most prominent in clinical settings, where the levels of catastrophizing are higher than e.g., in the general population. A clinical implication of this is, 
that it might be important to sereen people who present themselves with pain complaints to the general practitioner or a specialist with the Pain Catastrophizing Scale (PCS). Those at risk of these negative consequences, i.e., those with high levels of catastrophizing should be offered a treatment amed at reducing the level of catastrophing. This could be achieved by providing these individuals with a cognitive-behavioral therapy that e.g, starts with the rationale of the fear-avoidance model that was mentioned in the introduction section (Vlaeyen et al., 19956), challenges irrational ideas about pain, and uses behavior experiments. Indeed, there is preliminary evidence that such a specific therapy is highly effective (see e.g., Keefe et al 2004 ).

Second, pain catastrophizing, which has both trait-like and state-like qualities, is not an epiphenomenon of physical impairment and pain, but independently contributes to pain and pain-related consequences as mentioned above. Physical impaiment, also uniquely contributes to these consequences, albeit only modestly. A clinical implication of this is that in cases of substantial exacerbation of pain or pain-related disability, it might be advisable to have patients physically re-examined.

Third, catastrophizing in relation to a specific behavioral task (state catastrophizing) can be manipulated relatively easy. Therefore, in pain-rehabilitation programs healtheare professionals should be alert to catastrophic cognitions of patients in relation to specific exercises and should be trained in how to deal with these cognitions.

Fourth, in communily samples, a one-factor model of the PCS may be justifiable instead of a three-factor model. Therefore when the PCS is used for screening purposes in the general community, only the PCS total score should be used.

Fifth, in future research it would also be interesting to follow up on an extensive line of research that deals with the role of attentional factors in pain. There are indications that painrelated fear promotes inereased attention towards bodily sensations, including pain sensations, at the expense of other tasks, such as usual daily activities or the voluntary use of pain coping strategies (see Vlaeyen \& Linton, 2000). The specific role of pain catastrophizing in relation to this increased attention to or hypervigilance for painful stimuli was examined in a study by Crombez et al. (1998b). In this study, it was demonstrated that participants with high catastrophizing scores were hypervigilant for threatening somatic information (i.e., when expecting a painful stimulus). An interesting question then is whether catastrophizing negatively affects coping and adjustment to (chronic) pain by way of demanding attention to painful stimuli.

Finally, in general the studies presented here are a further confirmation of the growing notion that the role of pain catastrophizing in (chronic) pain is an important one and should not be underestimated. However, whether this role is causal in nature, as the frear-avoidance model of Vlaeyen et al. (1995a; 1995b) states, remains to be proven in further research. In this respect there is a need for new prospective and experimental studies that also take into account the role of potentially relevant variables such as neuroticism and depression. 


\section{References}

Aaronson, N. K. Acquadro, C.. Alonso, J, \& Apolone, G. (1992). International Qualty of Life Asseesmeth

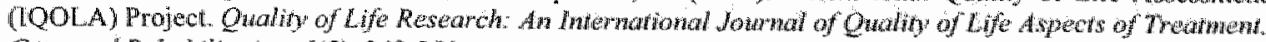
Care and Rehabilitration. $7(5), 349-351$.

Afteck, G., Tennen, H., Urrows, S. Higgins, P. (1992). Neuroticism and the pain-mod relation in rhematoid arthribs: Insights from a prospective daily study. Jounal of Consuling and Cincal Psyology. $60(1), 119-126$.

Andersson, H. I. (1994). The epidemiology of chronic pain in a Swedish rural area. Qualion of Life Research: An

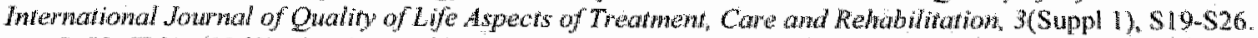

Anrep, G. V. (Ed), (1960). Conditioned Reflexes. An inwestigation of the Physiological Acriwy of the Cerebral Cortex by I.P. Pavlov. New York: Dower Publications, lnc.

Arniz, A. (1991). Principes en tectuieken van de cognitieve therapie. Directieve Therdpie, $1 /(4), 252-268$.

Arrindeli, W. A., \& Ettema, J. H. M. (1986). Hawdleiding bij een Modidimensionele Pspchopathologioindicator. Lisse: Swets \& Zeullinger.

Asmundson, G. J. G., Norton, P. J., \& Norton, G. R. (1999). Beyond paira: The rolle of fear and avoidance in chronicity. Clinical Psychology Review, 19(1),97-119.

Asmundson, G. J. G., \& Taylor, S. (1996). Role of anxiety sensitivity in pain-telated fear and avoidance. Journal of Behowional Medicine, $19(6), 577-586$.

Bandura, A. (1977). Self-efficacy: Toward a unifying theory of behavioral change. Pswehological Rewiew, 84(2), $191-215$.

Bantick, S. J., Wise, R. G., Ploghaus, A. Clare, S., Smith, S. M., \& Tracey, I. (2002). Inaging how attention modulates pain in humans using functional MRI. Brain, 125(Pt 2), 310-319.

Beck, A. T., Emory, G, \& Greenherg, R. L. (1985). Anxiery Disonders and Phobia. New York: Basic Books.

Beck, A. T., Rusli, A. J., Shaw, B. F., \& Emory, G. (1979). Cogritive Therapy of Depression. New York: Guilford Press.

Bédard, G. B. V., Reid, G. J., McGrath, P. J., \& Chambers, C. T. (1997). Coping and self-medication in a community sample of junior high school students. Pain Research and Management, 2(3), 151-156.

Beecher, H. K. (1959). Measurement of Subjective Responses. New York. Oxford University Press.

Bisgaard, T., Klarskov, B., Rosenberg, J., \& Kehlet, H. (2001). Characteristics and prediction of early pain after laparoscopic cholecystectomy. Paim, 90(3), 261-269.

Bishop, S. R., \& Warr, D. (2003). Coping, catastrophizing and chronic pain in breast cancer. Jonrinal of Behavioral Medicine, 26(3), 265-281.

Blyth, F. M. March, L. M., Brnabic, A. J., Jorm, L. R., Williamson, M., \& Cousins, M. J. (2001). Chronic pain in Australia: a prevalence study. Pain, $89(2-3), 127-134$.

Bowsher, D., Rigge, M. \& Sopp, L. (1991). Prevalence of clbronic pain in the British population: a telephone survey of 1037 households. The Part Cinic, 4(4), 223-230.

Boyd, D. B. (1989). Taxonomy and classification of pain. In C. D. Tollison (Ed.), Handbook of chronic paist management. (pp. 6-9). Baltimore, MD, USA: Williams \& Wilkins Co.

Bratuberg, G., Thorsilund, M. \& Wikman, A. (1989). The prevalence of pain in a general population. The results of a postal survey in a county of Sweden. Pain, 37(2), 215-222.

Buckelew, S. P., Conway, R. C., Shutty, M. S., Lawrence, I. A., Orafing, M. R., Anderson, S. K., Hewett, J. L., \& Keefe, F. J. (1992). Spontaneous coping strategies to manage acute pain and anxiety during electrodiagnostic studies. Arch Phys Med Rehabil, 73(6), 594-598.

Buer, N. \& Linton, S. J. (2002). Fear-avoidance beliefs and catastrophizing: occurrence and risk factor ir back pain and A.DT in the general population. Pain, $99(3), 485-491$,

Burns, I. W., Glem, B., Bruehl, S., Harden, R. N., R Lolland, K. (2003a), Cognitive factors influence outcome following multidisciplinary chronic pain treatment: a replication and extension of a cross-lagged pand analysis. Belwavor Research and Therapy, 4l(10), 1163-1182.

Burns, I. W., Kubilus, A., Bruehl, S., Harden, R. N., \& Lofland, K. (2003b). Do changes in cognitive factors influence outcome foilowing multidssciplinary treatment for chroric pain? A cross-lagged panel analysis. Joumal of Consulting and Clinical Psychology. 71(1),81-91.

Burton, A. K., Tillotson, K. M.. Main, C. J. \& Hollis, S. (1995). Psychosocial predictors of outcome in acente and subchronic low back trouble. Spime, 20(6), 722-72B.

Cats-Bariy, W. L., \& Frymoyer, J. W. (1991). The economics of spinal disorders. In J. W. Frymoyer, T. B. Ducker, N. M. Hadler, J. P. Kostuik \& In N. Weinstein (Eds.). The Adult Spine: Principles and Practice (Vol. Volume 1, pp. 85-105). New York: Raven Press.

Chaves, J. F, \& Brown, J. M. (1987). Spontaneous cognitive strategies for the control of clinical pain and stress. Joumal of Behanioral Medicine, 10(3), 263-276.

Colman, A. M. (Ed.) (1994). Companion Encyclopedia of Psychology (Vol. 1). London: Routledge. 


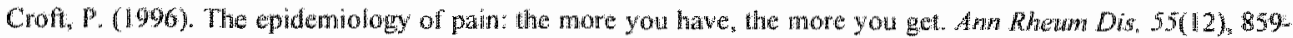
860.

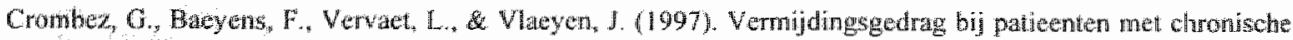
lage-rugpin. / Awordance behavior in patients with chronic low bedk pair. Gedrag and Gezondheid:

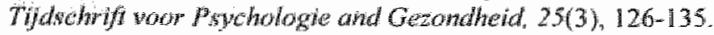

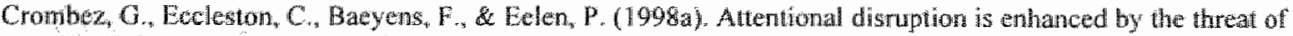
pain, Behawow Regearch and Therapy, 36(2). 195-204.

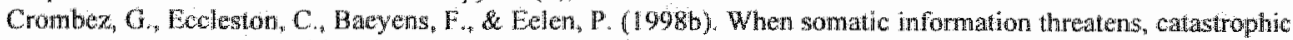
thinking enhaness anentional interfence. Pan, 75(2-3), $187-198$.

Crombo, G. Eccleston, C., Vlacyen, I. W., Vansteenwegen, D., Lysens, R., E Eelen, P. (2002). Exposure to physted movements in low back pain patients: restricted effects of generalization. Heath Psychot, 2J(6); 573578

Crombez, G., Hemans, D. Adriaensen, H. (2000). The emotional stroop lask and chronic pain: what is thresteming fot chande pain sufferers? Eur J Pain, $4(1), 37-44$.

Crombez, (H, Vervaet, I., Lysens, R., Baevens, F., \& Eelen, P, (1998c). Avoidance and confrontation of painful, back-atraning mowenents in chronic back pain patients. Behavior Modificonion. 22(1),62-77.

Crombez, $\mathrm{O}_{*}$ \& Vlacyen, I. W. S. (1996). The Pain Catastophizing Scale: Unpublished Dutch/Flemish Uransulution.

Crombez, G, Vtaeyen, J. W. S., Heuts, P. H. T. G. \& Lysens, R. (1999). Pain-related fiear is more disabting than pain itself: Evidence on the role of pain-related fear in chronic back pain disability. Pain, $80(1-2), 329-339$.

Crombie, I. K., Davies, H. T. O., \& Macrae, W. A. (1994). The epidemiology of chronic pain: Thme for new directons Poin, $57(1), 1-3$.

Crook, I., Rideout, E. \& Browne, G. (1984). The prevalence af pain complaints in a genewal population. Puin. $18(3), 299.314$

Derogatis, 1 . R., \& Cleary, P. A. (1977). Confirmation of the dimensional structure of the SCL-90: A study in construct validation. How rwal of Clinical Psychohogy. 33(4), $981-989$.

Derogatis, L. R., Lipman, $R$, S., \& Covi, L. (1973). SCL,90: an outpatien psychiatric rating scalempreliminaty "eport Pyychophamacology Bultetin, $9(1), 13-28$.

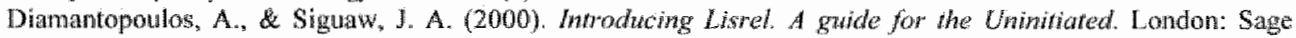
Publications.

Drossman, D. A., Leserman, J., L., Z, Keefe, F" ,Hu, Y. J., \& Toomey, T. C. (2000). Enects of coping on health outcome anong women with gastrointestinal disorders. Psychosom Med, 62(3), 309.317.

Dumkel-Schetter, C., Feinstein, L. G., Taylor, S. E. \& Falke, R. L. (1992). Patterns of coping with cancer. Heath Psychol, M(2), $79-87$.

Dunkel-Schetter, C., Folkman, S., L Lazarus, R. S. (1987). Correlates of social support receipt wourad of Personctity and Social Psychology, $53(1), 71-80$.

Dworkin, S. F., Von Korff, M., \& LeResche, L. (1990). Multiple pains and psychiatric disthbance. An epidemiologic investigation. Arch Gen Psychiony, 47(3), 239-244.

Eccleston, C, \& Cromber G. (1999). Pain demands attention. A cognitive-affective model of the intermuptive function of pain. Psychological Bwleth, $125(3), 356-366$.

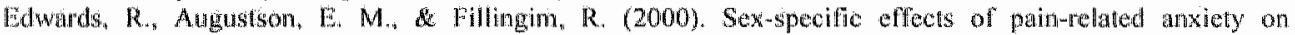

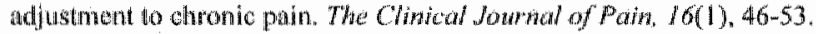

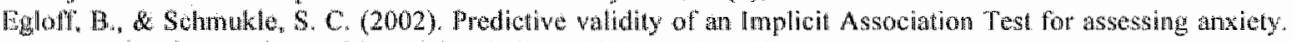
Jownot of Personaliry and Soctal Psychology, 83(6), 1441-1455.

Ellis. A. (1962). Rewson and Emotion in Psychothatupy. New Yonk: L., Ste Stuart.

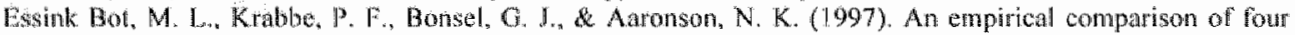
generio health status measures. Whe Nottingham Health Profile, the Medical Outcomes Study 36 -item ShortForm Hetih Survey, the COOP/WONCA charts, and the EuroQol instrument. Medical Care, 35(5), 522-537.

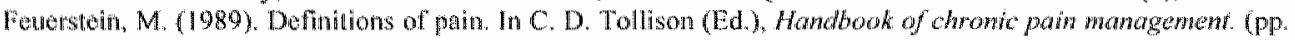
2.5). Ballimore, MD, USA: Williams \& Wilkins Co.

Foursten, M. Papciak, A. S, \& Hoon, P. E. (1987). Biobahavional mechanisms of chronic low back pain.

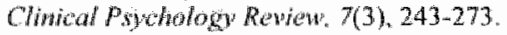

Flor, H. Behle. D. I., ae Bubumer. N. (1993). Assessment of pain-related cogritions in chronic pain patients. Behwowis Research and Therapy, 3h(1),63-73.

For $M_{n}$ \& Bitbaumer. N. (1994). Psychophysiological methods in the assessment and treatment of clironic musculoskeletal puin. Un J. G. Carlson \& A. R. Selfert (Eds.) Clinical applizd psychophysiology. Plenwh swes in bohavioral prychophysiology and mediche. (pp. 171-184). New York. NY. USA. Plenum Press.

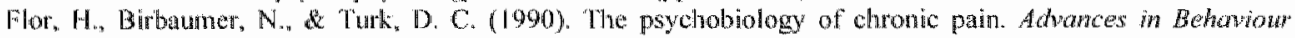
Rescanch and Therapy, 12(2), 47-84. 
Flor, H., Breitenstein, C., Birbaumer, N., \& Fuerst, M. (1995). A psychophysiological analysis of spouse solicitousness lowards pain behawiors, spouse interaction, and pain perception. Behawior Therapy. 26(2), $255-272$

Flor, H. Kerns, R. D. \& Turk, D. C. (1987). The role of spouse reinforcenent, perceived pain, and activity levels of chromic pain patients. Jonrnal of Psychosomatic Research, 37(2),251-259.

Flor, H., \& Turk, D. C. (1988). Chronic back pain and rheunatoid arthritis: Predieting pain and disabiliny from cognitive variables. Journal of Behavioral Medicme, 1/(3), $251-265$.

Flor, H., \& Turk, D. C. (1989). Psychophysiology of chronic paim: Do chronic pain patients exhibit symptomspecific psychophysiological responses? Psychologrical Bulletin, $105(2), 215-259$.

Flor, H. Turk, D. C., \& Rudy, T. E. (1989). Relationship of pain impact and significant other reinfotcentent of pain behawiors: The mediating role of gender, marilal status and marital satistaction. Poin, $38(1), 45,50$.

Folkman, S., Lazarus, R. S., Dunkel-Schetter, C., DeLongis, A., \& Gruen, R. J. (1986). Dyrammics of a stresstul encounter: cognitive appraisal, coping, and encounter outcomes. Jourmal of Personality ahd Sochal Psychology. 50(5), 992-1003.

Fordyce. W. E. (1976). Behavioral Methods for Chronic Pain and Mhexs. Saint Louis: The C.V. Mosby: Company.

Fordyce, W. E., Shetton, J. L., Dundore, D. E. (1982). The modification of awoidance learning pain behaviors. Journal of Behavional Medicine, 5(4), 405-414.

Gamsa, A. (1994a). The role of psychological factors in chronic pain: 1. A half century of study. Pain, $57(1), 5-$ 15.

Gamsa, A. (1994b). The role of psychological factors in chronic pain: Il. A critical appraisal. Pain, 57(1), 17-29.

Gaston-Johansson, F., Ohly, K. V., Fall-Dickson, J. M., Nanda, J. P., \& Kennedy, M. J. (1999). Pain, psychological distress, health status, and coping in paients with breast cancer seheduied for autotransplantation. Oncol Nurs Forkm, 26(8), 1337-1345.

Geisser, M. E., Casey, K. L. Brucksch, C. B., Ribbens, C. M., Appleton, B. B., \& Crofford, L. J. (2003). Perception of noxious and innocuous heat stimulation among healihy women and women with fibronyalgiat association with mood, somatic focus, and catastrophizing, Pain, $102(3), 243-250$.

Geisser M. E., Robinson, M.E \& \& Henson, C. D. (1994a). The Coping Strategies Questionnaire and chronic pain adjustment: a conceptual and empirical reanalysis. Climical Joumal of Pain. $10(2), 98-106$.

Geisser, M. E., Robinson, M. E., \& III, N. L. R. (1999). Pain beliefs, coping and adustment to chronic pain. Let's focus more on the negative. Pain Fontum, $8(4), 161-168$.

Geisser, M. E. Robinson, M. E., Keefe, F. J., \& Weiner, M. L. (1994b). Catastrophizing, depression and the sensory, affective and evaluative aspects of chronic pain. Pain. $59(1), 79-83$.

Giardino, N. D., Jensen, M. P., Turner, J. A., Ehde, D. M. \& Cardenas, D. D. (2003). Social cmiromment moderates the association between catastrophizing and pain among persons with a spinal cord injury. Poin. $106(1-2), 19-25$

Goossens, M. E. J. B. \& wan Tulder, M. W. (2000). Epideniologie van rugklachten. In II. W. S. Vlaeyen \& P. H. T. G. Heuts (Eds.), Gedragsgeariënteerde Behandelingsstrategieërn bif Rugpijn (pp. 1-14). Houten: Bolmn Stafleu Van Loghum.

Goubert, L., Crombez, G., \& Van Damme, S. (2004). The role of neuroticism, pain catastrophizing and painrelated fear in vigilance to pain: a structural equations approach. Puin. $107,234-241$.

Gouberi, L., Francken, G., Crombez, G., Vansteenwegen, D., de Lysens, R. (2002). Exposure to physical movement in chronic back pain patients: no evidence for generalization across different movements. Behaviour Research and Therapy, 40(4), 415-429.

Greenwald, A. G. MeGhee, D. E., \& Schwartz, J. L. (1998). Mcasuring individual differences in implicit

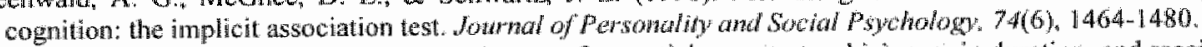

Groth Marnat, G., \& Fletcher, A. (2000). Influence of neuroticism, catastrophizing, pain duration, and receipt of compensation on short-term response to nerve block treatment for chronic back pain. Journal of Behavioral Medicine, 23(4), 339-350.

Haaga, D. A. (1992). Catastrophizing, confounds, and depression: A comment on Sulliwan and DEon (1990). Jownat of Abnormal Psycholagy. IOl(1), 206-207.

Hallberg, L. R., \& Carlsson, S. G. (1998). Anxiety and coping in patients with chronic work-related musculat pain and patients with fibromyalgia. Eur I Pain $2(4), 309.319$.

Hassett. A. L. Cone, J. D., Patella, S. I., \& Sigal, L. H. (2000). The role of catastrophizing in the pain and depression of women with fibromyalgia syndrome. Arthrifis Rheum, 43(11), 2493-2500.

Haythornthwaile, J. A. Clark. M. R., Pappagallo, M., \& Raja, S. N. (2003). Pair coping strategies play a role in the persistence of pain in post-herpetic neuralgia. Pain, $106(3), 453,460$.

Haythornhwaite, J. A., \& Heinberg. L. J. (1999). Coping with pain. What works, under what circumstances, and in what ways? Pain Forwm, $8(4), 172-175$. 
Haythornthwaite, I. A. Lawrence, I. W.. \& Fauerbach, I. A. (2001). Frief cognitive interventions for burn pain: Anw Behov Med, $23(1), 42-49$.

Heyneman, N. E., Fremouw, W. J, Garno, D., Kirkland, F. \& Heiden, L. (1990). Individual differences and the effectiveness of different coping strategres for pain. Cognitive Therapy and Research, $/ 4(1), 63-77$.

Hill, A. (1993). The use of pain coping strategies by patients with phantom limb pain. Pain, 55(3), 347-353.

Hill, A. Niven, C. A. \& Knussen, C. (1995). The role of coping in adjustment to phantom limb pain. Pain. $62(1) .769-86$.

Holmbeck, O. N. (1997). Tow ward terminological, conceptual, and statistical clarity th the study of mediators and moderatom: Examples from the child-elinical and pediatric psychology literatures. Joumal of Consulting and Clavical Psychology, 65(4), 599-610.

Jensen, M. P., Ehde, D. M., Hotrnan, A. J., Patterson, D. R., Cxemiecki, I. M., de Robinson, L. R. (2002). Cognitions, coping and social environment predict adjustment to phantom limb pain. Pain. 95(1-2), 133-142.

Jensen, M. P, \& Karoly, P. (1992). Self-report scales and procedures for assessing pain in adilts. In D. C. Turk \&. R. Melzack (Eds.), Handbook of pain assessment. (pp. 135.151). New York, NY. USA: The Guilford Press.

Jensen, M. P., Romano, J. M, fumer, J. A, Good, A. B., \& Wald, L. H. (1999). Patient belief's predict patient functioning: Further support for a cognitive-behavioural model of chronic pain. Pain, $8 /(1-2), 95-104$.

Jensen, M. P., Turner, I. A., \& Romano, J. M. (1992). Chronic pain coping measures: Individual vs. composide scores. Pain, $51(3), 273-280$.

Jensen, M. P., Tumer, J. A., \& Romano, J. M. (2001). Changes in beliefs, catastrophizing and coping are associated with improvement in multidisciplinary pain treatment. Jouprat of Consulting and Clinical Psychology, 69(4), 655-662.

Jensen, M. P., Turner, J. A., Romano, J. M., \& Karoly, P. (1991). Coping with chronic pain: A critical review of the literature Pain, 47(3), 249-283.

Joreskog, K. G., \& Sörbom, D. (1999), LISREL 8. 30. Chicago: Scientifie Software International, Inc.

Keefe, F. J., Brown, G. K., Wallston, K. A., \& Caldwell, D. S. (1989). Coping with rheumatoid arthritis pain: Catastrophizing as a maladiaptive strategy. Pain, $37(1), 5 \mid-56$.

Keefe, 1. J J Kashkar-Zuck, S., Robinson, E., Salley, A., Beaupre, P., Caldwell, D., Baucom, D., \& Hay thornthwaite, J. (1997). Pain coping strategies that predict patients' and spouses" ratings of patients' selfefficacy. Pain, 73(2), 191-199.

Keefe, F. J., Lefebvre, J. C., Egert, J. R., Affleck, G., Sullivan, M. J., \& Caldwell, D. S. (2000). The relationship ol gender to pain, pain behavior, and disability in osteoarthritis patients: the role of catastrophizing. Pain, $87(3), 325-334$

Keefe, F. J., Lefebvre, J. C., \& Smith, S. J. (1999). Catastrophizing research. Avoiding conceptual errors and maintaining a balanced perspective. Poin form, 8(4), 176-180.

Keefe, F. J., Lipkus, L, Lefebvre, J. C., Hurwitz, H., Clipp, E., Smith, J., \& Porter, L. (2003). The social context of gastrointestinal cancer pain: a preliminary study examining the relation of patient pain catastrophizing to patient perceptions of social support and caregiver stress and thegative responses. Pain, $103(1-2), 151-1.56$.

Keefe, F. J., Rumble, M. E., Scipio, C. D., Giordano, L. A., \& Perri, L. M. (2004). Psychological aspects of persistent pain: current state of the science. $J$ Paim, $5(4), 195-211$.

Kendall, P. C. (1992). Healthy thinking. Behowior Therapy, 23, 1-11.

Kerns, R. D. Turk, D. C, \& Rudy, T. E. (1985). The West Haven-Yale Multidimensional Pain Inventory (WHYMPD), Pain, 23(4), 345-356.

Klenerman, L., Slade, P. D., Stanley, I. M., Pennie, B., Reilly, J. P., Atchison, L. E., Troup, J. D. \& Rose, M. J. (1995). The prediction of chronicity in patients with an atcute attack of low back pain in a general practice setting. Spine, $20(4), 478-484$.

Knussen, $C_{\text {. }}$ Lee, D. (1998), cluronic fatigue syndrome: Symptoms, appraisal and ways of coping. British Journal of thealth Psychology, 3, 111.121 .

Latham, J." \& Davis, B. D. (1994). The socioeconomic inpact of chronic pain. Discibility and Rehobilitation: An

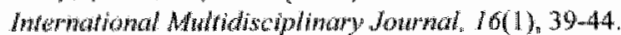

Lazarus, R., \& Folkman, S. (1984), Siress, Appraisal, and Coping. New York: Springer.

Lefebvre, J. C. \& Keefe. F. J. (2002). Memory for pain: the relationship of pain cattastrophizing to the recall of datly meumatoid arthritis pain. Climical ifownal of Pain, 18(1), 56-63.

Lefebvire, J. C., Lester. N... \& Keefe, $\mathbb{F}$. J. (1995). Pain in young adults: II. The use and perceived effectiveness of pain-coping strategies. Clinical Journat of Pain. $1 /(1), 36-44$.

Lester, N., Lefebvre, J. C. \& Keefe, F. J. (1996). Pain in young adults--III: Relationships of three patin-coping measures to pain and activity interference. Clinical Journal of Pain. /2(4), $291-300$.

Lethem, J. Slade, P. D., Troup, J. D., \& Bentley, G. (1983). Outline of a fear-avoidance model of exaggeraied pain perception: 1. Behowiour Research ard Therapy, 2/(4),401-408. 
Lilienfeld, S. O. (1996). Anxiety sensitivity is not distinct from trat anxiety. In R. M. Rapee (Ed.) Currewt Controversizs in the Anxiety Disorders (pp. 241-227). New York: Guilford.

Linton, S. J., Buer, N., Vlaeyen, J., \& Hellsing. A. L. (2000). Are fear-avoidance beliefs related to the inception of an episode of back pain? A prospective study, Psychology and Health $14(6), 1051-1059$.

Linton, S. J., \& Hallden, K. (1998). Can we screen for problematic back pain? A screening questionnaire for predicting outcome in acute and subacute back pain. Clinical fournal of Pain, 14(3), 209-215.

Lousberg, R. (1994). Chronic Pain. Multiaxial Assessment and Behavioril Mechanisms, Rijksuniversiteit Limburg, Maastricht.

Magni, G., Caldieron, C. Rigatti Luchini, S., \&e Merskey, H. (1990). Chronic musculoskeletal pain and depressive symptoms in the general population: An analysis of the Ist National Heallh and Nutrition Examination Survey data, Paim, 43(3), 299-307.

Main, C. I. \& Waddell, G. (1991). A comparison of cognitive measures in low back pain: Statistical structure and clinical validity at initial assessment. Pain, 46(3), 287-298.

Martin, M. Y., Bradley, L. A., Alexander, R. W., \& Alarcon, G. S. (1996). Coping strategies predict disability iin patients with primary fibromyalgia. Pain. $68(1), 45-53$.

McCracken, L. M., Goetsein, V. L. \& Semenchuk, E. M. (1998). Coping with pain produced by physical activity in persons with chronic low back pain: immediate assessment following a specific pain event. Behow Med. 24(1), 29-34.

McMurray, G. A. (1950). Experimental study of a case of insensitivity to pain. Archives of Nenrology and Psychiatr, 64, 650-667.

Meichenbaum, D. (1977). Cognitive Behanior Modificarion. New York: Plenum Press.

Meichenbaum, D. (1985). Srress Inoculation Training. New York: Perganon Press.

Melzack, R., \& Casey, K. L. (1968). Sensory, motivational, and central contol determinants of pain. A new conceptual model. In D. R. Kenshalo (Ed). The Skin Senses (pp. 423-443). Springtield, Illinois, U.S.A.: Charles C Thomas.

Melzack, R., \& Torgerson, W. S. (1971). On the language of pain. Anesthesiology, 34(1), 50-59.

Melzack, R., \& Wall, P. D. (1965). Pain mechanisms: a new theory. Science, $150(699)$ ), $971-979$.

Melzack, R., \& Wall, P. D. (1982). The Challenge of Pain. Bungay, Suffolk: Richard Clay (The Chancer Press) L.td.

Mersky, H. (1986). Classification of chronic pain, descriptions of chronic pain syndromes and definitions of pain terms. Pain, suppl. 3, S10-S11, S13-S24, S217.

Miller, R. P., Kori, S. H., \& Todd, D. D. (1991). The Tampa Scale: Unpublished Report.

Miller, T. W., \& Kraus, R. F. (1990). An overview of chronic pain. Hospiral and Community Psychiatry 4l(4), 433-440.

Nathan, P. W. (1976). The gate-controll theory of pain. A criticall review. Braint a journat of newrology, (1),

123-158.
Newton, C. R., \& Barbaree, H. E. (1987). Cognitive changes accompanying headache treatment: The use of at thought-sampling procedure. Cognvitive Therapy and Research, $17(6), 635-651$.

Osman, A., Barrios, F. X., Gutierrez, P. M., Kopper. B. A., Merritield, T.. \& Grittmann, L. (2000). The Pain Catastrophizing Scale: Futher psychometric evaluation with adult samples. Journal of Behovioral Medicine.

23(4), 351-365.
Osman, A. Bartios, F. X., Kopper, B. A. Hauptmann, W., Jones, J., \& O Neill, E. (1997) Vactor structure reliability, and validity of the Pain Catastrophizing Scale. Jourial of Behawional Aedicine, 2061.589.605. Public Policy Perspectiwes. Washington, D.C.: National Academy Press.

Parry, C. B. (1980). Pain in avulsion lesions of the brachial plexus. Pain $9(1), 41-53$.

Peters, M. L., Vlaeyen, J. W. van Drunen, C. (2000). Do tibronyalgia patients dasplay hypervigilande for imnocuous somatosensory stimuli? Application of a body scanning reaction time paradigm. Path, 86(3), 283292.

Petrak, F., Hardt, J., Kappis, B., Nickel, R., \& Tiber Egle, U. (2003). Determinants of health-related quality of life in patients with persistent somatoform pain disorder. EuF J Pain, $7(5), 463-471$.

Pfingsten, M., Hildebrandt, J., l.eibing, E., Franz, C.. \& Saur, P. (1997). Effectiveness of a multimodal treatment program for chronic low-back pain. Pain $73(1), 77-85$.

Philips, H. C. (1987). Avoidance behaviour and its role in sustaining chronic pain. Behowour Research and Theropy, 25(4), 273-279.

Picawet, H.S. \& Schouten, J.S. (2003). Musculoskeletal pain in the Netherlands: prewalences, consequences, and risk groups, the DMC(3)-study. Pain, 102(1-2), 167-178.

Picavet, H. S., Vlaeyen, I. W., \& Schouten, J. S. (2002). Pain catastrophizing and kinesiophobiai predictors of chronic low back pain. Am J Epidemiol, 156(11), 1028-1034 
Pieavet, H. S. U., Sohoutem, J. S. A. G., \& Smit, H. A. (1996). Prevalenties en consequenties van lage nuglachten. in hel MORGEN-project 1993-1995 (263200004). Bilthowen: RIVM.

Picavet, H. S. J., van Gils, H. W. V. \& Schouten, J. S. A. G. (2000). Klachten wan het bewegingsapparaat in de Nederlandse bevolking. Prevalenties, consequenties en risicogroepen (266807 002). Bilthowen: RIVM.

Ploghtus, A. Narain, C., Beckmam, C. F. Clare, S., Bantick, S., Wise, R., Mathews, P. M., Rawlins, J. N.. \& Tracey, 1. (2001). Exacerbation of pain by anxiety is assockated with activity in a hippocampal network. $d$ Neturosel, $2 /(24), 9896-99093$.

Ploghaus, A, Tracey, 1., Clare, S., Gati, J. S., Rawins, J. N, \& Mathews, P. M. (2000). Leearning about pain: the neural substrate of the prediction error for aversive events. Proc Nall Acad Sici U SA. 97(16), 9281-9286.

Price, D. D. (1988). Classical and current theories of pain mechanisms, Psychological and Neural Mechanisms of Pain (pp. 212-231). New York: Raven Press.

Radanow, B. P., Sturzenegger, M. (1996). Predicting recovery from common whiplash. Eur Neural, 36(1), 48 51.

Reesor, K. A., \& craig, K. D. (1988). Medically incongruent chronic back pain: Physical limitations, suffering, and ineffective coping. Paim, 32(1), 35-45.

Reiss, S. (1991). Expectancy model of fear, anxiety, and panic. Clinical Psychology Review, $/ 1(2), 141-153$.

Robinson, M. E., Riley, J. L., 3rd, Myers, C. D., Sadler, I. J., Kvaal, S. A. Geisser, M. E., \& Keefe, F. J. (1997), The Coping Strategies Questionnaire: a large sample, item level factor analysis. Clinical Joumal of Pain, $13(1), 43-49$.

Roland, M. \& Motris, R. (1983). A study of the natural history of back pain. Part I: development of a reliable and sensitive measure of disability in low-back pain. Spine, $8(2), 141-1.44$.

Romano, J. M., Turner, J. A., Jensen, M. P., Friedman, L. S., \& et al. (1995). Chronic pain patient-spouse behawional interactions predict patient disability. Paim, $63(3), 353-360$.

Rosenstie $I_{s}$ A. K., \& Keefe, $F^{*}$. J. (1983). The usc of coping strategies in chronic low back pain patients: Relationship to patient characteristics and current adjustment. Pain, $17(1), 33-44$.

Rudy, T. E., Turk, D. C., Brena, S. F., Stieg, R. L., \& et al. (1990). Quantification of biomedical findings of chronic pain patients: Development of an index of pathology. Paim 42(2), 167-182.

Rutter, D. R., Iconomou, G., \& Quine, L.. (1996), Doctor-patient communication and outcome in cancer patients: An intervention. Psychology and Health, 12(1), 57-71.

Salkovskis, P. M., \& Clark, D. M. (1990). Affective responses to hyperventilation: A test of the cognitive model of panic. Behaviour Research and Therapy, 28(1), 51-61.

Samwel, H., Slappendel, R., Crul, B. J., Voerman, V. F. (2000). Psychological predictors of the effectiveness of radiofrequency lesioning of the cervical spinal dorsal ganglion (RF-DRG). Eur J Pain, 4(2), 149-155.

Santavirta, N., Biorvell, H., Koivumaki, E., Hurskainen, K., Solovieva, S., \& Konttinen, Y. T. (1996). The factor structure of coping strategies in hemophilia. J Psychosom Res, 40(6), 617-624.

Santavirta, N., Bjoryell, H., Solovieva, S., Alaranta, H. Hurskainen, K., \& Konttinen, Y. T. (2001). Coping strategies, pain, and disability in patients with hemophilia and related disorders. Arthritis Rheum, 45(1), 4855 .

Schanberg, L. E., Anthony, K. K., Gil, K. M., Lefebvre, J. C., Kredich, D. W., \& Macharoni, L. M. (2001). Family pain history predicts child health status in children with chronic rhe umatic disease. Pediotrics. $108(3), \mathrm{E} 47$.

Schneider, 1 , \& Karoly, P. (1983). Conceptions of the pain experience: The emergence of multidimensional models and the ir implications for contemporary clinical practice. Clinical Psychology Review, 3(1). 61-86.

Severeijns, R. wan den Hout, M. Vlacyen, J., \& Picavet, H. (2002). Pain catastrophizing and general health status in sf farge Dutch community sample. Pain, $99(1-2), 367-376$.

Severeijns, R., van den Hout, M. A., Vlacyen, J, W. S. (in press). The causal status of pain catastrophizing, an experimental test with healthy participants. Eur J Pain.

Severelijns, R., Vlaeyen, J., van den Hout, M., \& Picavet, H. (2004). Pain catastrophizing is associated with healith indices in museuloskeletal pain: A cross-sectional study in the Dutch community. Health Psychology. $23(1), 49-57$

Severeijns, R., Vlaeyen, J. W. S., van den Hout, M. A. \& Weber, W. E. J. (2001). Pain catastrophizing predicts pain intensity, disability, and psychological distress independent of the level of physicat impaiment. Climical Journat of Pain, $17(2), 165-172$.

Shapiro, D. E., Boggs, S. R. Melaned, B. G., \& Graham Pole, J. (1992). The effect of varied physician affect on recall, anxiety, and perceptions in women al risk for breast cancer: An analogue study. Healh Psychology, $1 /(1), 61-66$

Spanos, N. P.. Radike Bodorik, H. L., Ferguson, J. D., \& Jones, B. (1979). The effects of hypnotic susceptibility, suggestions for andgesia, and the utilization of cognitive strategies on the reduction of pain. Joturnal of Abnormal Pswhology, 88(3), 282-292. 


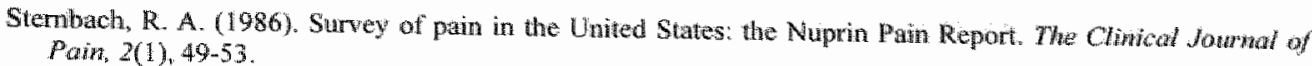

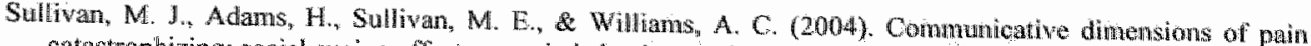
catastrophizing? sockal cueing effects on pain behaviour and coping. Parn, $107(3), 220-226$.

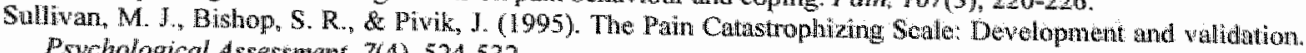
Psychologrical Assessment, $7(4), 524-532$

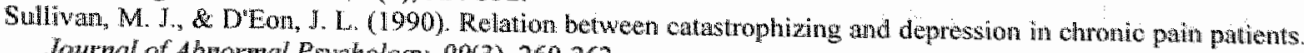
Jownal of Abrownal Psychology, 99(3), 260-263.

Sulliwan, M. I., \& Neish, N. (1999). The eflects of disclosune on pain during dental bygane treatment: the moderating role of catastrophizing. $F a n, 79(2-3), 155-163$.

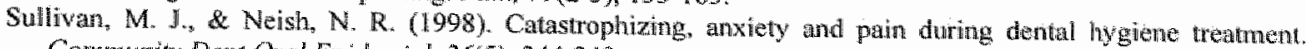
Commuwity Dent Oral Eprdemiol, $26(5), 344-349$.

Sulwan, M. J., Rodgers, W. M. \& Kirsch, 1. (2001 a). Catastrophixing, depression and expectancies for pain and emotional distress. Pain, $91(1-2), 147-154$.

Sullian, M. J. Rodgers, W. M., Wilson, P. M., Bell, G. J., Muray, T. C., ar Fraser, S. N. (2002). Ar experimental investigation of the relation beween catastrophizing and activity intolerance. $P$ chis, $100(1-2)$, 47. 53 .

Sullivan, M. I., Rouse, D., Bishop, S., \& Johnston, S. (1997). Thought suppression, catastrophizing, and pain. Cognitive Therapy and Research $2 \int(5), 555-568$.

Sulliwan, M. J., Stanish, W., Waite, H., Sullivan, M., R Tripp, D. A. (1998). Catastrophizing pain, and disability in patient with soft-tisste injuries. Pain, $77(3), 253-260$.

Sullivan, M. J., Thorn, B., Haylhonthwaite, J. A., Keefe, F., Martin, M. Bradiey, L. A., Lefebve, J. C. (2001b). Theoretical perspectives on the relation between catastrophizing and pain. Chwical Nownat of Paw 17(1). 52-64.

Sulliwan, M. J., Tripp, D. A., Rodgers, W. M., \& Stanish, W. (2000a). Catustrophizing and pain perestion in sport participants. Journal of Applied Spom Psychohogy. 12(2), 1,51-167.

Sullivan, M. J., Tripp, D. A., Santor, D. (2000b), Gender diferences in pain and pain behavion: The role of catastrophizing. Cognirive Thercapy and Research, 24(1). 121-134.

Tan, G., Jensen, M. P." Robinson Whelen, S., Thomby, J. I, \& Monga, T. N. (2001). Coping with chronic pain: a comparison of two measures. Pain, $9(1-2), 127-133$.

Thastum, M., Zachariae, R., \& Herlin, T. (2001). Pain experience and pain coping strateges in chidren with juvenile idiopathic artinitis. o Rhewmatol. 28(5), 1091-1098.

Thastum, M., Zachariae, R., Scholer, M., Bjerring, P., \& Herlin, T, (1997). Cold pressor pam: cariparing tesponses of juvenile anthrits patients and their parents. Scand J Rhemmatol, 26(4), 272-279.

Thon, B. e., Rich, M. A., \& Boothby, J. L. (1999). Pain beliefis and coping attempts. Conceptual model building. Pain Forum, $B(4), 169.171$.

Tunk. D. C. (1990). Customizing treatment for chronic pan patients: who, what, and why, The clivical Jowryal of Pain, $6(4), 255 \mathrm{~m} 270$.

Turk, D. C. (1996a). Biopsychosocial perspective on chronic pain. In R. 1. Gatchel \& D. C. Turk (Lds.). Psychological approaches to pain management A practionerts handbook (pp. 3-32), New York. NY, USA: The Guilford Press.

Turk, D. C. (1996b). Cognitive factors in chrone pain and disability, In K. S. Dobson \& K. D. Craig (Eds.).

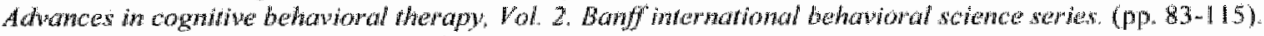
Thousand Oaks, CA, USA: Sage Publications, lno.

Turk, D. C., \& Ferandez, E (1991). Pain: A cognitive-behavioural perspective. In M. Watson (Ed.). Comeer

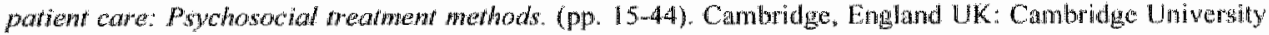
Press; Leicester England UK: British Psychohogical Society.

Turk, D. C, \& For, H. (1984). Etiological heories and treatments for chronic back pain: II. Psychological models and interventions. Poin, $19(3), 209-233$.

Turk, D. C. Meichentaum, D., Ge Genest, M. (1983). Pain: a cognitive-behavional perspectwe, Pain am

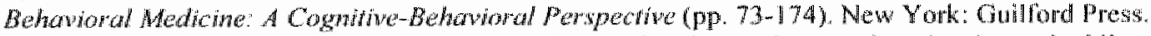

Turk, D. C. \& Rudy, T. E. (1986). Assessment of cognefive fachors in chronic pain: A worthwhife anterprise? Jownal of Conswiting and Chintal Psychologe. 54(6), 760-768.

Turk, D. C. \& Rudy, T.E. (1988). Toward an empirically derived taxonony of atwonic pain patients: Integertion of psychological assessment diata. Jownat of Consuling and Clinical Psychology, 56(2), 233-238.

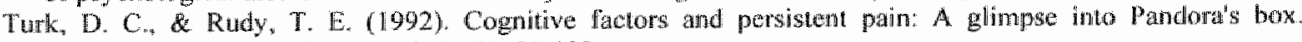
Cogmine The apy and Research. $16(2), 99122$.

Turmet, J. A. \& Aaron, li.. A. (2001). Pain-related catastrophizing: what is in? Chincal joumat of pam, The. 17(1), $65-71$. 
Turner. J. A. Clancy. S. (1986). Suteghes for coping with chronic low back pain: Relationship to pain and disabilify. Fon, $24(3), 355-364$.

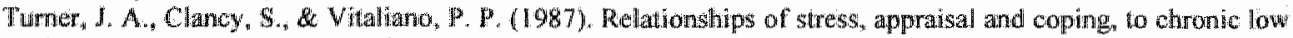
brick pain. Bewawiou Researh and Therapy. $25(4), 281.288$.

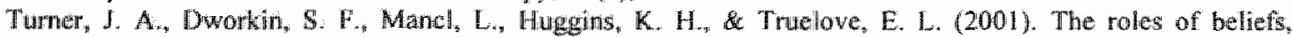
catastrophizing, and coping in the functionimg of patients with temporomandibular disorders. Poin. $92(1-2)$, 41.51.

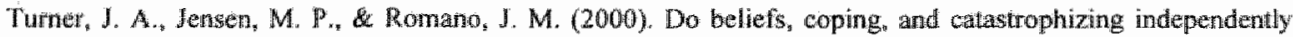
predict fumotioning in patents with chtonic pain? Pain, $85(1-2), 115-125$.

Tumer, J. A. Jensen, M. P. Wamis, C. A, \& Cardenas, D. D. (2002). Catastrophizing is associated with pain untensily, psychological distress, and pain-related disability among individuals with chronic pain after spinal cord injuy, pain, $98(1-2), 127-134$

Unwin, M, Symmons, D., Allisom, T, Brammah, T., Dusby, H., Roxby, M., Simmons, A., Rillams, G. (1998). Estimating the burden of musculoskeletal disorders in the community: the comparative prevalence of symptoms al differentinatomicall sites, and the relation to social deprivation. Ann Rhew Dis, 57(11), 649m. 655.

Van Damme, s, Crombez, G., Bijtebiet, P., Couburt, L., \& Van Houdenhove, B. (2002a). A confirmatory factor andysils of the Pan Catastrophizing Seale: invariant factor structure across clinical and non-chinical populations. Pain, 96(3), 319-324.

Van Damme, $\$$., Crombez, $G_{6}$ \& Eccleston, $\mathrm{C} .(2002 \mathrm{~b})$. Retarded disengagement from pain cues: the effects of pain catastwophizing and pain cxpectancy. Pawn, $100(1-2), 111-118$.

Van Damme, S., Crombez, G., Vlaeyen, J. W. S., Goubet, L., Broeck, A. v. d., \& Houdenhove, B. V. (2000). De Pain Catastrophizing Scale: psychometrische karakteristieken en normering. Gedragstherapic, $33(3), 209$. 220 .

van den Hout, J. H., Vlaeyen, J. W., Peters, M. I., Engethard, I. M., \& van den Hout, M. A. (2000). Does Cailume hurt? The effects of failure feedback on pain report, pain tolerance and pain avoidance. Eur $P_{\text {aif }}, 4(4), 335$ 346.

van der Zee, K. I., Sanderman, R, weyink, J. W. (1993). De psychometrische kwaditeiten wan de MOS $36-$ item Short Form Health Survey $\left(\mathrm{SF}_{-3} \mathrm{G}\right)$ in een Nedertandse populatie. Tydschrift woor Sociale Gezond heddszorg, $71,183-191$.

van der Zee, K. I. Sanderman, R., Heyink, J. W. \& de Haes, H. (1996). Psychometric qualities of the RAND 36-item thealth survey 1.0: a multidimensional measure of general health status. Imernorionat Joumal of Behavioral Medicine, 3(2), 104-122.

wan Dulmen, A. M., Fennis, J. F. M., Mokkink, H. G. A., van der Velden, H. G. M., 8 et al. (1995). Doctordependent changes in complaint-related cognitions and anxiety during medical consultations in functional abdominal complaints. Pspchological Medicine, 25(5), 1011-1018.

van Tulder, M. W. Koes, B. W., B Bouter, L. M. (1995). A cost-ofullness stady of back pain in The Netherlatinds. Pain, 62(2), 233-240.

van Tulder, M. W., van Breukelen, G., Koes, B. W., \& Bouter, L. M. (1997). De kosten van mugklachten in Neriorland: een matschappelijk probleem? Medisch Contact, 52, 829-832.

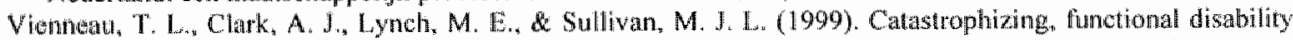
and pain raports in aultits with chronic low back pain. Pan Reseanch and Monagemen, 4(2), 93-96.

Vlacyen, 1. W. S., de Jong, J., Geillen, M., Heuts, P. H. T, G., van Brevketen, G. (2001). Graded exposure in vivo in the treatment of pain-related ferr. A replicated single-case experimental design in four patients with chitonic low back pain. Dehavious Rescarch and Therapy, 39(2), $151-166$.

Vloven, J. W. S., Gistuts, S. M. Kole Snijoers, A. M., \& Schuerman, J. A. (1990), What do chronic pain

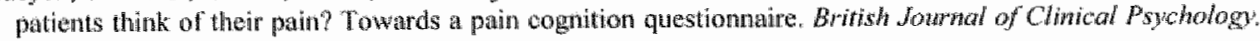
$29(4), 383-394$.

Wheyen, I. W. S., Kole Snijders, A. M. J.; Boeren, R. G. B. \& van Eek, H. (I995a) Fear al movemend(ro)injury in chronic low back pain and its relation to behavioral performance. Pain. $62(3)$ ). 363372

Vhasen, J. W. S, Kole Snijders, A. M. J., Rotteveel, A. M., Re Ruesink, R. (1995b). The role of rear of movement(re)injury in pain disability, Journal of Octupafional Rehabilitotion, 5(4), 235-252.

Vlaeyen, J. W. S. \& Linton, S. J. (2000). Feap-avoidance and its consequences in chronic musculoskeletal pain: A state of the art Pain. $85(3), 317-332$

Vlaeyen, J. W. S., Seelen, H. A. M. Peters, M., de Jong, P., Aretz, E., Beisiegel, E., \& Weber, W. E. I. (1999). Fear of movemend(re)injury and muscular reactivity in chronic low back pain patients: An experimentat investigetion, Pain, $82(3), 297-304$.

Von Korf, M. Dworkin, S. F, \& le Resche, L. (1990). Graded chronic pain status: An epidemiologie evaluation. Pan, $40(3), 279 \mathrm{~m} 291$ 


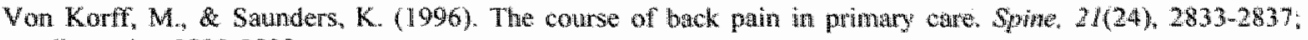
discussion $2838-2839$.

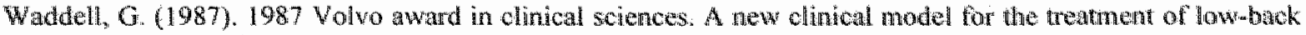
pain. Spine, $12(7), 632-644$.

Waddell, $G_{2}$ \& Main, C. J (1984). Assessment of severty in low-back thsorders. Sphne, 92), $204-208$.

Waddell, G., Main, C. J., Morris, E. W. Di Paola, M., \& Gray, I. C. (1984). Chronic low -hack pain, psychologic distress, and illness behavior. Spme. $9(2), 209-213$.

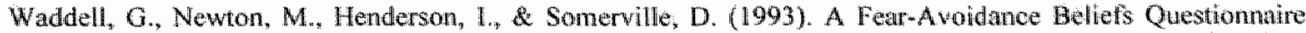
(FABQ) and the role of fear-avoidance beliefs in chronic low back pain and disabillty. Pam, $32(2), 157-168$.

Waddell, G. Pilowsky, I., \& Bond, M. R. (1989). Clmical assessment and interpretation of abnormal illnoss behaviour in low back pain. Pain, 39(1), 41-53.

Watson, D., Clark, L. A., \& Tellegen, A. (1988). Development and validation of hrief measures of positive and negative affect: The PANA S scales. Jownal of Personality and Social P.ychology. $54(6), 1063-1070$.

Weisenberg, M. (1994), cognitive aspects of pair. In P. D. Wall \& $\mathbb{R}$. Melzack (Eds) The Texbook of Pan (3rd ed, pp. 275-289). Edinburgh: Churchill Livingstone.

Wells, N. (1994). Perceved control over pan. Relation to distress and disability. Resedwh in Nursing and Healih, $17(4), 295-302$

Westemann, L., Spies, K., Stahl, G., Hesse, F. W. (1996). Relatiwe effectiveness and validity of mood "nduction procedures: a meta-analysis. European Jow nal of Social Psyohology, 26,557-580.

WHO. (1980). The Consequences of Disease, Intemational Classificaton of Imparmments, Disabilitias, and Handicaps (pp. $23-43$ ). Genewa: World Health Organization.

WHO (2001). Introduction, $1 C F$; Inernational Classification of functianing Disability and Healit (pp. 1-25). Geneva: World Teatth Organization. 


\section{Summary}

Musculoskeletal pain is a very common health problem, with reports of chronic musculoskeletal pain ranging from $4 \%$ to even as much as $44.4 \%$ in the general community. Evidence is cumulating that psycho-social factors may be important in the development and maintenance of pain-related problems, such as disability, depression, quality of life, health care utilization, loss of productivity. This thesis focuses on one of these factors: pain catastrophizing. The studies in this thesis address the possible role of pain catastrophizing in (chronic) pain.

Chapters 1,2 , and 3 provide a general introduction, in which some widely used pain-related terms are defined, epidemiological data are discussed, and a historical overview of the role of psychological factors in (chronic) pain us given. Some conceptual models are discussed that have greatly influenced current conceptualizations of pain, treatment of pain, and research on pain. From this discussion the main theme of this thesis is introduced: pain catastrophizing. A proposal for a definition of pain catastrophizing is followed by a discussion of some conceptual issues and research findings. Finally, the outline of this thesis is presented along with the research questions.

Chapter 4 addresses the question whether pain catastrophizing is related to pain intensity, disability, and psychological distress, independent of the level of physical impairment and whether these relationships are the same for different pain subtypes. It was found that there were significant associations between pain catastrophizing and these variables, independent of the levell of physical impairment. The latter however also made a unique, though modest, contribution to the prediction of pain intensity and disability. No differences between pain subtypes were found in this respect. Finally, it was demonstrated that there was no relationship between pain catastrophizing and physical impairment.

Chapter 5 describes a cross-sectional population-based study in which the relationship between pain catastrophizing and general health status was examined as well as the factor structure of the Pain Catastrophizing Scale (PCS). The results indicated that across different pain subtypes, pain catastrophizing was particularly associated with mental health, general health perception, social functioning, and vitality. Furthermore, the results confirmed a threefactor structure of the PCS, although a one-factor structure was found justifiable as well. The three-factor structure (as well as the one-factor structure) was invariant across different pain subgroups.

Chapter 6 presents the results of a cross-sectional population-based study that examined the relationship between pain catastrophizing and several health indices: specialist consultation, use of pain medication, and absenteeism or work disability. It was shown that for people with a current episode of musculoskeletal pain, pain catastrophizing, but also pain intensity and the presence of multiple pain locations was significantly associated with these variables.

Chapter 7 addresses the question whether pain catastrophizing contributes to the development of chronic musculoskeletal pain over a six months period and to the prediction of specialist consultation, absenteeism, and use of medication. The results demonstrated that people with musculoskeletal pain (current and recent pain combined) who catastrophized about the pain, had a higher risk of developing chronic pain complaints over a six months period, as compared to people who did not catastrophize about the pain. Also, we found that the risk of developing chronic pain complaints over a six months period increased if people reported to have more pain problems. The significant cross-sectional relationships between pain catastrophizing and the health indices (see Chapter 6) were not confirmed prospectively. The relationship between catastrophizing and chronicity on the one hand and the health 
indices on the other hand was not moderated by the number of pain complaints nor by pain imtensity.

Chapters 8 and 9 describe two experimental studies in which the causal status of pain catastrophizing in (chronic) pain was examined. In both experiments the level of catastrophizing was experimentally manipulated. It was expected that temporarily inducing. the level of catastrophizing in healthy participants (Chapter 8), would increase the level of expected and experienced pain during a cold pressor task and would result in a significant shorter duration of ice-water immersion. Contrary to what was expected, none of the predicted effects reached significance, although the attempt itself to manipulate the level of catastrophizing was successful. In the second experiment (Chapter 9), an attempt was made to temporarily reduce the level of catastrophizing in patients with chronic recurrent low back pain. It was expected that a reduction in pain catastrophizing would result in a better perfomance on a knee-extension-flexion task (KEF-task) on a Biodex system 3. The results demonstrated that the attempt to reduce the level of catastrophizing was successful when we limited the analyses to participants with high catastrophizing scores. Participants in the experimental group, where catastrophizing was successfully reduced, performed better on two KEF-task parameters. However, it could not be conclusively demonstrated that the reduction in catastrophizing contributed to this better performance.

Chapter 10 provides a summary and a general discussion of the main results. Some strengths and limitations of the studies are discussed. Some practical implications and suggestions for further research are given. 


\section{Samenvatting}

Muskuloskeletale pijn is een veel voorkomend grezondheidsprobleem wathif prevalentiecijfers van chronische muskuloskeletale pijn varieren van $4 \%$ tot maar liefst $44.4 \%$ in de algemene populatie. Er is groeiend bewijs dat psychosociale factoren een belingrijke roll kumnen spelen bij het ontwikkelen en in standhouden van pijngerelateerde problematiek, zoals pijngerelateerde beperkingen, depressie, kwaliteit van leven, medische consumptie en verlies van productiviteit. Dit proefschrift concentreert zich op én wan deze factoren: catastroferen over pujn. De onderzoeken in dit proefschrift zijn toegespitst op de mogelijke rol van catastroferen over pijn in relatie tot (chronische) pijn.

Hoofdstukken 1, 2 en 3 bevatten een algemene introductie waarin een antal veel gebruikte pijngerelateerde begrippen wordt gedefinieerd, epidemiologische gegevens worden besproken en een historisch overzicht wordt gegeven van de rol van psychologische factoren in relatie tot (chronische) pijn. Een aantal conceptuele modellen die van grote invloed zijn geweest op de huidige opvattingen over pijn, behandeling van pijn, alsmede onderzoek naar pijn, wordt besproken. Vanuit deze bespreking wordt het hoofdthema van dit proefschrift geintroduceerd: catastroferen over pijn. Een voorstel voor een definitie van catastroferen over pijn wordt gevolgd door een discussie van enkele conceptuele kwesties en onderzoeksresultaten. Tenslotte worden de opzet en de onderzoeksvragen van het proefschrift gepresenteerd.

Hoofdstuk 4 behandelt de vraag of er, onafhankelijk van de mate van lichamelijk letsel, een rellatie is tussen catastroferen over pijn enerzijds en pijnintensiteit, beperkingen en psychisch onwel bevinden anderzijds en de vraag of deze relaties hetzelfde zijn voor verschillende subtypen pijn. Uit de onderzoeksresultaten bleek een significant verband tussen catastroferen over pijn en deze variabelen, onafhankelijk varn de mate van lichamelijk letsel. De mate van lichamelijk letsel droeg echter wel, zij het in beperkte mate, bij aan het voorspellen van pijnintensiteit en de mate van beperkingen. Er werden geen versehillen gevonden tussen subtypen pijn in dit opzicht. Tenslotte werd aangetoond dat er geen relatie is tussen catastroferen over pijn en de mate van lichamelijk letsel.

Hoofdstuk 5 beschrijft een cross-sectioneel populatie onderzoek waarin de relatie tussen catastroferen over pijn en algemene gezondheidstoestand werd onderzocht alsmede de factorstructuur van de Pain Catastrophizing Scale (PCS). Uit de resultaten bleek dat voor verschillende subtypen pijn catastroferen over pijn vooral samenhangt met mentale gezondheid, algemene gezondheidsbeleving, sociaal functioneren en vitaliteit. Voorts bevestigden de resultaten een drie-factoren structuur van de PCS alhoewel een één-factor structuur ook verdedigbaar bleek te zijn. De drie-factoren structuur van de PCS (maar ook de éen-factor structurur) was invariant voor verschillende pijn subgroepen.

Hoofdstuk 6 beschrijft de resultaten van een cross-sectioneel populatie onderzoek warin het verband tussen catastroferen over pijn en verschillende gezondheidsindicatoren werd onderzocht: specialisten bezoek, gebruik van pijmmedicatie en ziekteverzuim of arbeidsongeschiktheid. Bij mensen met een huidige episode van muskuloskeletale pijn werd een significant verband gevonden tussen catastroferen over de pijn, pijnintensiteit en het hebben van meerdere pijnklachten enerzijds en deze variabelen anderzijds.

In hoofdstuk 7 wordt de vraag behandeld of catastroferen over pijn bijdratagt aan het ontwikkelen van chronische pijnklachten over een periode van zes maanden en aan het voorspellen van specialisten bezoek, ziekteverzuim en het gebruik van pijnmedicatie. De resultaten toonden aan dat mensen met muskuloskeletale pijn (huidige pijn en recente pijn gecombineerd) die catastroferen over de pijn een hoger risico hebben om chronische pijnklachten te ontwikkelen ower een periode van zes maanden in vergelijking met mensen die niet catastroferen over de pijn. Ook vonden we dat het risico op het ontwikkelen van chronische pijnklachten toeneemt bij mensen die meer pijnklachten rapporteren. De 
significante cross-sectionele relaties tussen catastroferen over pijn en de gezondheidsindicatoren (zie hoofdstuk 6) werden prospectief niet bevestigd. De relatie tussen enerzijds catastroferen en anderzijds het chronisch worden wan de pijnklachten en de gezondheidsindicatoren (specialistenbezoek, ziekteverzuim en gebruik van pijnmedicatie) Werd niet gemodereerd door het aantal pijnklachten, noch door de intensiteit van de pijn.

In hoofdstuk en 9 worden twee experimenten beschreven waarin de causale rol van catastroteren bij (chronische) pijn werd onderzocht. In beide experimenten werd de mate van catastroferen over pijn experimenteel gemanipuleerd. De verwachting was dat het tijdelijk verhogen vain de mate van catastroferen over pijn bij gezonde deelnemers aan het experiment (hoofdstuk 8) zou leiden tot een verhoogde mate van werwachte en ervaren pijn tijdens cen zogenaamde "cold pressor" taak en zou resulteren in een kortere onderdompelingstijd. In tegenstelling tot hetgeen verwacht werd, waren geen van de voorspelde effecten significant alhoewel de poging zelf om de mate van catastroferen te manipuleren succesvol was. In het tweede experiment (hoofdstuk 9) werd een poging gedaan om de mate van catastroferen tijdelijk te werminderen bij patiënten met chronische recurrente lage rugp ijn. De verwachting was dat vermindering van de mate van catastroferen over pijn zou leiden tot een betere prestatie op een knie-extensie-flexie taak (KEF-taak) op een Biodex system 3. De resultaten toonden aan dat de poging om de mate van catastroferen te verlagen alleen succesvol was als we de analyses beperkten tot de deehnemers met hoge catastroferen scores. Deelnemers in the experimentele groep, waar de mate van catastroferen stccesvol gereduceerd werd, presteerden beter op twee KEF-taak parameters. Het kon echter niet afdoende aangetoond worden dat de vermindering in catastroferen bijdroeg aan deze betere prestatie.

Hoofdstuk 10 bevat een algemene discussie van de belangrijkste empirische bevindingen en een overzicht van de methodologisch sterke punten en de methodologische beperkingen. Er worden een aantal praktische implicaties en suggesties voor verder onderzoek gegeven. 


\section{Dankwoord}

Eindelijk is het dan zover: het proefsclurift is af! De afgelopen jaren zijn omgevlogen, jaren waarin ik veel heb geleerd over wetenschappelijk onderzoek en wetenschappelijk jargon, catastroferen, efficiènt werken, schrijven en (vooral) schrappen, plezier en frustratie, vasthoudendheid, geduld en nog veel meer. Rest mij nog op deze plaats een kort, doch zeer welgemeend woord van dank uit te spreken aan iedereen die aan deze bijzondere leerervaring heeft bijgedragen.

Allereerst Johan Vlaeyen, mijn co-promotor in de afgelopen jaren. Beste Johran, je inhoudelijke kennis en belezenheid op het gebied van pijn is werkelijk indrukwekkend. De nust die je altijd uitstraalde, zeker wanneer de reviewers weer eens lastig deden, heeft menig catastrofale gedachte verholpen. Onze discussies over de aard en de rol van catastroteren waren interessant, leerzaam en bovenal plezierig. Een van die discussies is zelfs uitgemond in een topical review in PAIN. Johan, bedankt!

Marcel van den Hout, mijn promotor in de afgelopen periode. Beste Marcel, hoewel rijd een schaars goed voor je is heb ik dat nooit gemerkt wanneer we een afspraak over mijn onderzoek hadden. Je bevlogenheid en enthousiasme voor onderzoek hebben aanstekelijk gewerkt. Je methodische, modelmatige en theoretische manier van denken bewonder $i k$. Marcel, bedankt voor je wijze adviezen!

Dan zijn er nog mensen wier bijdrage aan het tot stand komen van dit proefschrift vooral in praktische en ondersteunende zin onmisbaar was. Allereerst natuurlijk alle deelnemers aan de verschillende studies. Hun bereidheid om, vaak vele, vragenlijsten in te vullen en zich vol vertrouwen te onderwerpen aan soms minder plezierige procedures zoals bijwoorbeeld de cold pressor taak, is bewonderenswaardig. Erik Schouten wil ik bedanken omdat hij nooit te beroerd was om ook mijn meest triviale statistische vragen geduldig, en in zelfs foor mij begrijpelijke taal, te beantwoorden. Maria Ummels bedank ik ondat ze de hele logistiek en uitvoering van het experiment met de cold pressor taak voor haar rekening heeft genomen. De participanten waren erg onder de indruk van haar optreden! Susan Picavet, verbonden aan het Rijksinstituut voor Volksgezondheid en Milieu (RIVM) dank ik voor haar nuttige adviezen en de plezierige samenwerking met betrekking tot de drie populatie studies. Lieven Danneels, verbonden aan de afdeling Revalidatiewetenschappen en Kinesitherapie van het Universitair Ziekenhuis te Gent, bedank ik voor de coördinatie van het experiment met de Biodex. Zonder zijin hulp en inspanningen was het experiment er nooit gekomen. Sam Bynens en Steve Janssens, vrijgevestigde fysiotherapeuten en destijds bezig met een vervolgopleiding aan bovengenoemd universitair ziekenhuis, bedank ik voor de uiterst prettige samenwerking en de zorgvuldige uitvoering van het experiment met de Biodex. Het heeft hun menig vrije zaterdag gekost! De regelmatige bezoeken aan Gent waren muttig, leerzaam en niet in het minst, erg gezellig.

Een woord van dank ook aan de collega's van de afdeling Medische Psychologie en het Departement Medische Klinische en Experimentele Psychologie voor hun morele ondersteuning en aan de goede vrienden van mijn intervisie clubje die naast hun wetenschappelijke interesse vooral ook veel gevoel voor humor hebben. De twee-maandelijkse intervisiebijeenkomsten waren en zijn een welkome bron van ontspanning.

Walter Arts en Gerdy Konings, bedankt dat jullie bereid zijn om mij tijdens het verdedigen van mijn proefschrift terzijde te staan. Jullie aanwezigheid is een belangrijke steun in de rug. 
In de laatste maar zeker niet de minste plaats gaat mijn dank uit naar mijn ouders die mij altijd gesteund hebben en natuurlijk naar Petra, Myrthe en Daphne die mij doen realiseren dat er belangrijkere dingen in het leven zijn dan promoveren. 


\begin{abstract}
About the author
Rudy Severeijns was bom November 16, 1961 in Heer (Maastricht), the Netherlands. In 1980 he obtained his VWO diploma (highschool) at the "St. Maartenscollege" in Mastricht and started his study Psychology at the University of Nijmegen. He specialized in Clinical Psychology, completed the main curriculum Industrial Psychology, and graduated in 1987. Subsequently, he accomplished his alternative national service at the policlinic outpatients' department of psychiatry of the "Reinier de Graaf Gasthuis" in Delft, whereupon he was appointed as a psychologist and worked there until March 1995. During this period he started a post-university education behavior therapy, as well as the route to registration as a clinical psychologist of the Dutch Institute of Psychologists (NIP). In 1993 he was officially registered as a clinical psychologist NIP and in 1994 as a behavioral therapist of the Association for Behavior Therapy and Cognitive Therapy (VGCl). Since April 1995 he is working as a clinical psychologist at the department of Medical Psychology of the Maastricht University Hospital. In 1998 he was officially registered as a supervisor for the VGCt and in 1999 as a health care psychologist. He started his Ph.D. in 1999.
\end{abstract}




\section{Publications}

Severeijns, R., Vlaeyen, J.W.S., van den Hout, M.A., Picavet, H.S.I. (2005). Pain catastrophizing and consequences of musculoskeletal pain: a prospective study in the Dutch community. Joumal of Pain, 6(2), 125-132.

Severeijns, R., Wlaeyen, J.W.S., \& van den Hout, M.A. (2004). Do we need a communal coping model of pain catastrophizing? An alternative explanation. Pain $111,226-229$.

Severeijns, R., Vlaeyen, J.W.S., van den Hout, M.A., \& Picavet, H.S.J. (2004). Pain catastrophizing is associated with health indices in musculoskeletal pain: a cross-sectional study in the Dutch community. Health Psychology 23, 49-57.

Severeijns, R, van den Hout, M.A., Vlaeyen, J.W.S., \& Picavet, H.S.J. (2002). Pain catastrophizing and general health status in a large Dutch community sample. Pain 99, 367376.

Severeijns, R., Vlaeyen, J.W.S., van den Hout, M.A., \& Weber, W.E.J. (200I). Pain catastrophizing predicts pain intensity, disability, and psychological distress independent of the level of physical impaiment. The Clinical Joumal of Pain 17, 165-172.

Arts, W.J.I.M., \& Severeijns, J.R.M. (1998). Complicaties bij de cognitieve behandeling van dwangstoornis. Directieve Therapie 18(4), 366-377.

Van Gemert, W.G., Severeijns, R., Greve, J.W.M., Groenman, N.H., \& Soeters, P.B. (1998). Psychological functioning of morbidly obese patients after surgical treatment. International Jownal of Obesity 22, 393-398.

Severeijns, J.R.M., \& Arts, W.J.J.M. (1996). De behandeling wan drie patiënten met een beroepsgebonden hondenfobie. Directieve Therapie 16(3), 260-273.

Arts, W., Severeijns, R., Haan, E. de, Hoogduin, C.A.L., \& Hoogduin, W. (1993). Motiveringstechnieken bij de ambulante behandeling van dwangstoornissen mef behulp van exposure in vivo en responspreventie. Directieve Therapie 13,27-48.

Hoogduin, C.A.L., \& Severeijns, J.R.M. (1992). Diagnostiek bij de conversiestoonis: mythen en richtlijnen. Directieve Therapie $12,361-367$.

Korrelboom, C.W., Kernkamp, J.H.B., Hoogduin, C.A.L., \& Severeijns, R. (1992). Paniekmanagement en het voorspellen van outcome bij agorafobie. Een empirische verkenning. Gedragstherapie 25, 183-200.

Arts, W., Severeijns, R., Hoogduin, C.A.L., Haan, E. de, \& Schaap, C. (1990). De Inventarisatielijst Dagelijkse Bezigheden (IDB): Een replicatieonderzoek naar betrouwbaarheid en validiteit. Gedragsherapie 23, 17-27.

Severeijns, R. \& Haan, E. de. (1990). Het geheim van mevrouw Jansen. Direchleve Therapie, 10, 16-22. 